\section{SANDIA REPORT}

SAND99-0162

Unlimited Release

Printed February 1999
MS 0619 Reviewand ApprovalDesk, 15102 (1) For

A 3D Lattice Boltzmann Code for Modeling Flow and Multi-Component Dispersion

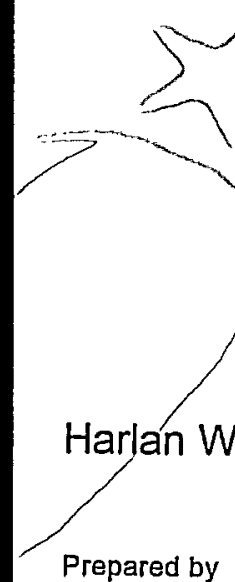

Sandia National Laboratories

Albuquerque, New Mexico 87185 and Livermore, California 94550

Sandia is a multiprogram laboratory operated by Sandia Corporation

a Lockheed Martin Company, for the United States Department of

Energy under Contráct DE-AC04-94AL85000.

Approved for public release; further dissemination unlimited.

in Sandia National Laboratories
REQEIVEO

MAR 121999

OSTI 
Issued by Sandia National Laboratories, operated for the United States Department of Energy by Sandia Corporation.

NOTICE: This report was prepared as an account of work sponsored by an agency of the United States Government. Neither the United States Government, nor any agency thereof, nor any of their employees, nor any of their contractors, subcontractors, or their employees, make any warranty, express or implied, or assume any legal liability or responsibility for the accuracy, completeness, or usefulness of any information, apparatus, product, or process disclosed, or represent that its use would not infringe privately owned rights. Reference herein to any specific commercial product, process, or service by trade name, trademark, manufacturer, or otherwise, does not necessarily constitute or imply its endorsement, recommendation, or favoring by the United States Government, any agency thereof, or any of their contractors or subcontractors. The views and opinions expressed herein do not necessarily state or reflect those of the United States Government, any agency thereof, or any of their contractors.

Printed in the United States of America. This report has been reproduced directly from the best available copy.

Available to DOE and DOE contractors from

Office of Scientific and Technical Information

P.O. Box 62

Oak Ridge, TN 37831

Prices available from (703) 605-6000

Web site: http://www.ntis.gov/ordering.htm

Available to the public from

National Technical Information Service

U.S. Department of Commerce

5285 Port Royal Rd

Springfield, VA 22161

NTIS price codes

Printed copy: A08

Microfiche copy: A01

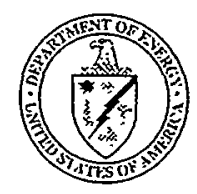




\section{DISCLAIMER}

Portions of this document may be illegible in electronic image products. Images are produced from the best available original document. 
Unlimited Release

Printed February 1999

\title{
A 3D Lattice Boltzmann Code for Modeling Flow and Multi-Component Dispersion
}

\author{
Harlan W. Stockman \\ Geochemistry Department \\ Sandia National Laboratories \\ P.O. Box 5800 \\ Albuquerque, NM 87185-0750
}

\begin{abstract}
This report documents the JB_PH lattice Boltzmann code, a program for modeling multi-component flow and dispersion. The lattice Boltzmann method is reviewed and methods are compared for increasing the efficiency of the algorithms. Accuracy of the code is tested with four benchmark problems: (1) permeability of a simple cubic array of spheres; (2) Taylor-Aris dispersion between infinite plates; (3) dispersion in a duct; and (4) dispersion in a simple cubic array of spheres. The memory and speed requirements of the method are assessed in the context of the benchmarks. The code structure and purpose of each major function are described, and instructions are given for compilation.
\end{abstract}




\section{Acknowledgments}

The author thanks the Sandia LDRD program for providing support for the tuning and testing of the lattice Boltzmann algorithms described in this report. I thank my colleagues in Organizations 6100 and 9100 for their support and stimulating discussions. Andrew Glew of Intel and William Kahan of University of California, Berkeley, provided helpful suggestions on the denormal problem. 


\section{Contents}

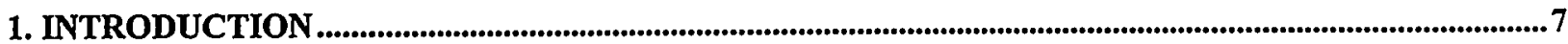

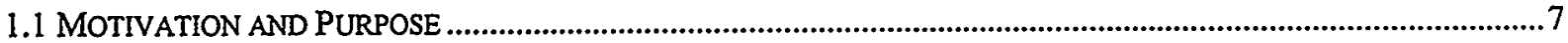

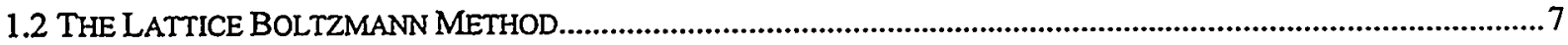

2. COMPUTATIONAL EFFICIENCY

3. ACCURACY

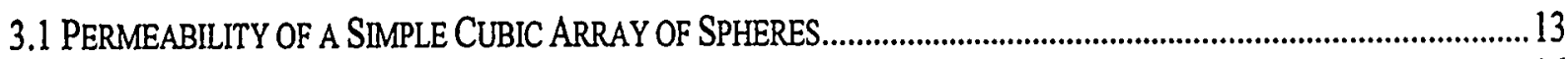

3.2 WALL CONDITIONS: TAYLOR-ARIS DISPERSION BETWEEN INFINITE PLANAR SHEETS ........................................16

3.3 TAYLOR DISPERSION IN A DUCT WITH RECTANGULAR CROSS-SECTION ….......................................................... 18

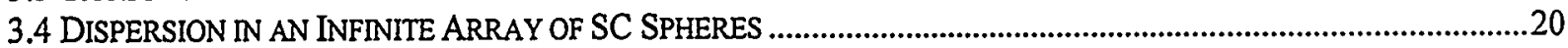

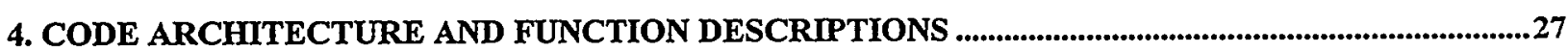

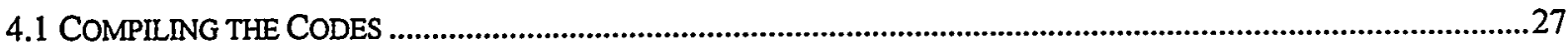

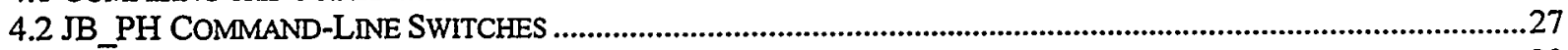

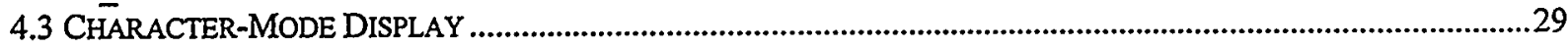

4.5 PASSING DATA TO ASYNCHRONOUS GRAPHICS PROGRAMS: PIOTFILE. BIN ……..............................................29

4.6 EXAMPLE OF PLOTFIIE . BIN USAGE: A WIN95/NT INTERFACE .......................................................................30

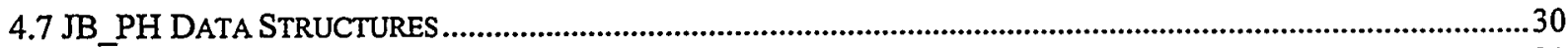

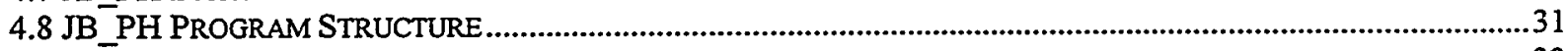

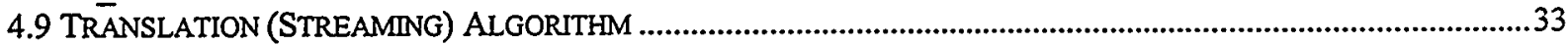

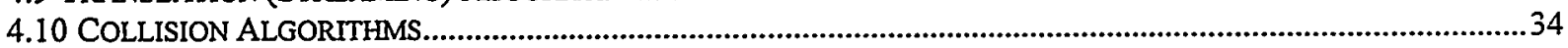

4.11 CONTROLLING FLUID SPEED AND GRAVITY ADJUSTMENTS .............................................................................34

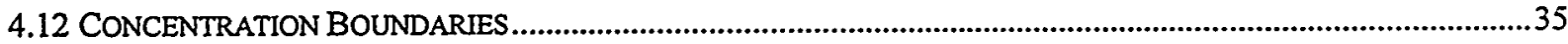

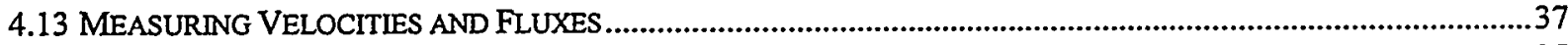

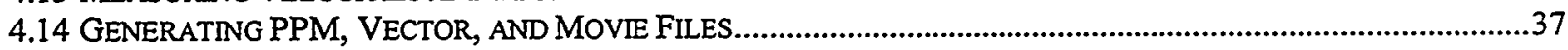

4.15 MEASURING DISPERSION: THE MEAN_STD. TXT FILE ..........................................................................................40

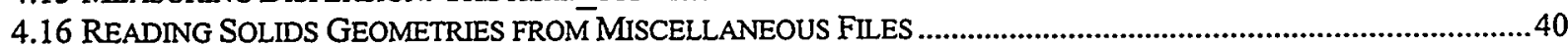

4.17 GENERATING PSEUDO-RANDOM SPHERICAL PACKINGS ....................................................................................4

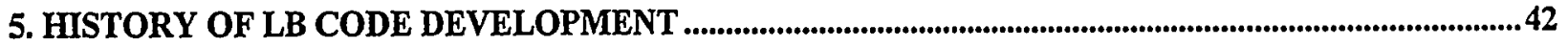

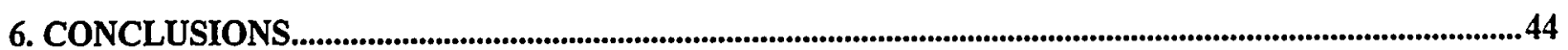

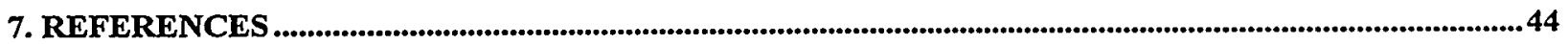

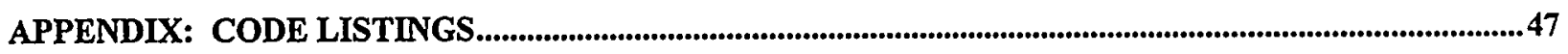

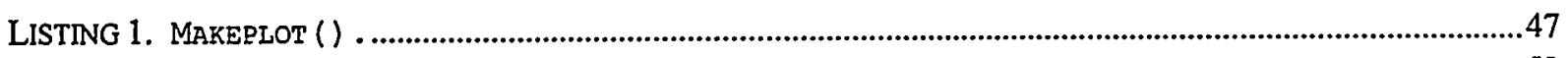

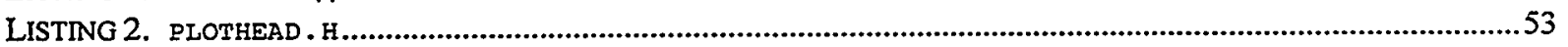

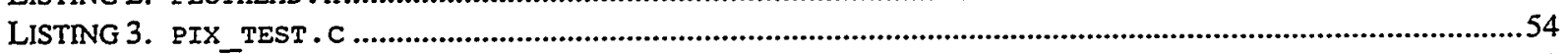

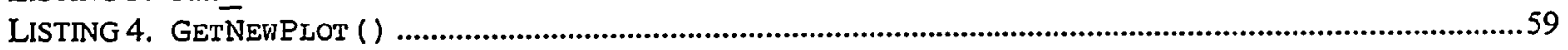

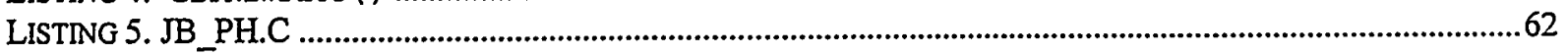




\section{Figures}

FigURE 1. THE 19 VECTORS OF THE D3Q19 MODEL. THERE IS A "REST" PARTICLE AT $(0,0,0)$, THERE ARE 6 VECTORS WITH WEIGHT $1 / 18^{\text {Th }}$ ALONG THE CARTESIAN AXES, AND 12 VECTORS WITH WEIGHT $1 / 36^{\text {TH }}$ IN THE $X-Y, Y-Z$ AND $X-$ $Z$ PLANES.

FIGURE 2. SIMPLIFIED COLLISION FUNCTION FOR ONE 3D 6-VECTOR TRACER, $\partial U / \partial T=0$.

FIGURE 3. SIMPLE CUBIC (SC) GEOMETRY. AT LEFT IS A SMALL SECTION OF AN ARRAY. RIGHT, A FACE OF THE UNIT CUBE, SHOWING THE ENTRACE TO A CHANNEL; THE "IDEAL" SPHERICAL SECTIONS ARE SHOW IN RED, AND THE PIXELATION FOR $X=12(R=6)$ ARE SHOWN BY THE BLACK OUTLINE.

FIGURE 4. LB ESTIMATE OF PERMEABILITY OF SC ARRAY OF SPHERES, AS FUNCTION CELL EDGE $X=2 R$. THE DEVIATION FROM THE ZICK AND HOMSY ${ }^{13}$ VALUE IS DUE MAINLY TO THE PIXELATION EFFECT SHOWN IN FIGURE 3.

FIGURE 5. LB NORMALIZED PERMEABILITY AND SPHERE RADII FOR THE NON-TOUCHING SPHERES, $R=X / 3$

FIGURE 6. TAYLOR-ARIS DISPERSION BETWEEN INFINITE PARALLEL PLATES. THE TEST GEOMETRY IS AT LEFT; A SLUG OF SOLUTE IS INJECTED INTO AN ESTABLISHED, LEFT-TO-RIGHT POISEIULLE FLOW. AS THE SLUG SPREADS, THE PROJECTION OF CONCENTRATION ONTO THE $X$-AXIS EVENTUALLY BECOMES GAUSSIAN. AT RIGHT IS THE ERROR FOR TWO TYPES OF WALL CONDITIONS; TRADITIONAL BOUNCE-BACK AND A NO-FLUX CONDITION (ERROR IS DIFFERENCE BETWEEN EQUATIONS 9 AND 10).

FIGURE 7. GEOMETRY FOR DISPERSION IN A RECTANGULAR DUCT.

FIGURE 8. COMPARISON OF LB CALCULATIONS FOR DISPERSION IN A RECTANGULAR DUCT, AGAINST THE PREDICTIONS OF CHATWIN AND SULLIVAN. ${ }^{20}$

FIGURE 9. LB FLOW FIELDS FOR $R E=150$ (TOP) AND $R E=4.1$ (BOTTOM), FOR THE PLANE AT FRACTIONAL DEPTH $Z=$ 0.0694 (2.5 LATTICE UNITS). FOR $P E=132$, THE CORRESPONDING $S C$ ARE 0.88 (TOP) AND 32 (BOTTOM). VECTOR LENGTHS ARE $4{ }^{\text {TH }}$-ROOT SCALED TO EMPHASIZE REGIONS OF LOW-SPEED FLOW. THE TRUE DISTRIBUTION OF SOLIDS IS INDICATED BY THE CROSS-HATCH PATTERN; THE DASHED CIRCLE IS THE IDEAI SPHERICAL SECTION FOR THIS $Z$ POSITION.

FIGURE 10. SOLUTE PATTERNS IN THE EARLY STAGES $\left(T / T_{C}<0.3\right.$ ) OF THE LB CALCULATIONS, FOR $S C=0.88$ (TOP) AND $S C=32$ (BOTTOM); BOTH ARE AT $P E=132$. THE STEP NUMBER IS INDICATED ON THE UPPER RIGHT OF EACH FRAME. EACH FRAME SHOWS CUTS AT FRACTIONAL DEPTHS $Z=0$ AND $Z=1 / 2(0$ AND 18 LATTICE UNITS, RESPECTIVELY). THE SOLUTE IS INDICATED IN BLACK; INTENSITIES ARE SQUARE-ROOT SCALED TO EMPHASIZE LOWER CONCENTRATIONS. THE LOW-CONCENTRATION RIPPLES IN THE $T=48$ FRAMES ARE NUMERICAL ARTIFACTS THAT ARE QUICKLY DAMPED.

FIGURE 11. COMPARISON OF LB RESULTS WITH EXPERIMENTS AND THEORY. GUNN AND PRYCE ${ }^{23}$ PONTS (OPEN CIRCLES) REPRESENT GAS DISPERSION EXPERIMENTS

FIGURE 12. A SLUG OF SOLUTE INJECTED BETWEEN WALLS OF A ROUGH FRACTURE. EACH FRAME SHOWS A SINGLE TIMESTEP (INDICATED IN BLACK IN THE UPPER RIGHT CORNER), AND TWO CUTS THROUGH THE FRACTURE. THE SOLIDS ARE IN WHITE; THE INITIAL CARRIER FLUID IS RED; AND THE INITIAL SLUG IS BLUE FLUID. AT THE TOP OF THE FRAME IS A SUB-HORIZONTAL CUT, MIDWAY BETWEEN THE BOUNDING SURFACES, AT Y=NROW/2; AT THE BOTTOM OF EACH FRAME IS AN ORTHOGONAL CUT, AT $z=$ NLAY / 2. THE SLUG WAS READ FROM A BITMAP VIA THE READBMP4 SLUG () FUNCTION, AND THE WALLS OF THE FRACTURE WERE READ WITH THE FRACTALSUREACE () FUNCTION.

FIGURE 13. IMAGES OF THE RAYLEIGH-TAYLOR INSTABILITY, PLOTTED WITH THE FUNCTION SAVE3DPPM(). A DENSER BLUE FLUID INITIALLY OVERLIES A RED FLUID; THE TWO OVERTURN IN MUSHROOM-SHAPED PATTERNS. THE TIMESTEP IS SHOWN AT THE BOTTOM OF EACH FRAME.

FIGURE 14. A PSEUDO-RANDOMIZED SOLIDS PATTERN CREATED BY THE SETFCCSOLIDS () FUNCTION. THE SOLIDS ARE IN WHITE, AND THE COLORS SHOW AN EQUILIBRIUM FLOW FIELD, WITH THE LOWEST FLOW SPEEDS CORRESPONDING TO THE "COOLEST" COLORS (E.G. BLACK), AND THE WARMEST COLORS (RED) CORRESPONDING TO HIGHEST FLOW SPEEDS.

\section{Tables}

TABLE 1. PERFORMANCE FOR LB ALGORITHMS (MUPS = MILLIONS SITE UPDATES PER SECOND)...........................12 


\section{Introduction}

\subsection{Motivation and Purpose}

In the geosciences and biological sciences, and in chemical and thermal engineering, it is often desirable to perform accurate calculations of flow and dispersion in complex geometries. Most groundwater contaminants are dilute tracers, and dispersion describes the spreading of these tracers as aqueous and gaseous solutions move through porous and fractured rocks and soils. There is much interest in developing numerical methods that allow one to scale from microscopic flow at the scale of a pore or fracture, through the scale used in lab experiments, to flow on the scale of kilometers. ${ }^{1,2,3}$ Medical engineers attempt to model the dispersion of contaminants in the human lung, ${ }^{4}$ where nature imposes an inherent compromise on the Peclét number $(P e \sim 100)$; flow must be fast enough to ensure an adequate oxygen supply to the lungs, but not so fast that there is little time to exchange oxygen and carbon dioxide. In chemical engineering, solid catalysts are often in the form of porous beds, and the reaction of chemicals on the surfaces of the particles is influenced by the dispersion of solutes. To achieve the greatest efficiency, flows through the packed beds are often at modest to high Reynolds number $(R e)$, a realm that is difficult to model by traditional methods. In thermal engineering, the forced cooling of a microprocessor on a circuit board typically involves flow through extremely complex geometries, and the removal of heat by diffusion and advective spreading, at $R e$ and $P e$ numbers $\sim 1000$. Current approaches to modeling these phenomena ${ }^{5}$ typically involve hybrid methods, grafting constant temperature flow fields onto simplified thermal models for the fluid solid interface. For such convoluted geometries with simultaneous flow and transport, direct, whole-system application of traditional methods, such as finite elements, is simply too costly or cumbersome.

This report describes the development of a 3D lattice Boltzmann (LB) method for modeling flow and dispersion through complex geometries at $R e$ and $P e$ from 0 to $\sim 1000$. The code is stable with arbitrarily complex solid-fluid interfaces, and in special cases, reaches site update rates of 2.6 million per second on a single-processor Pentium II computer. The accuracy and speed of the code have been tested for several geometries of increasing complexity. While these geometries are not as complex as catalytic beds, they are amenable to analytic solutions or comparisons with more conventional numerical methods and experiments.

\subsection{The Lattice Boltzmann Method}

The LB method breaks physical space into a large number of nodes or sites $\left(\approx 10^{6}\right.$ in the simulations described in this report); at each node is a set of mass probability distributions. In each time step, the distributions translate from node to node along fixed velocity vectors, then undergo simple collisions that conserve physical properties such as momentum. The collision operator is designed so that the particle velocity distributions at each node obey the Navier Stokes equations and the advection-dispersion equations.

The LB method, and the BGK simplification, are well-described by Qian et al. ${ }^{6}$ The evolution of the particle distribution function $f_{i}$ satisfies

$$
f_{i}\left(\mathbf{x}+\mathbf{e}_{i}, t+1\right)=f_{i}(\mathbf{x}, t)-\left(f_{i}(\mathbf{x}, t)-f_{i}^{e q}(\mathbf{x}, t)\right) / \tau
$$


where $\mathbf{x}$ is a node position in the lattice, $\mathbf{e}_{i}$ is one of $i$ allowed velocities $(e . g . i=0,1, \ldots, 18$ in D3Q19 model $^{6}$ ), $t$ is the time step, $f_{i}^{e q}$ is the equilibrium distribution, and $\tau$ is the BGK relaxation parameter, which determines the viscosity and diffusion coefficients. In typical LB computer programs, a translation or streaming function performs the left side Eq. (1), and a collision function performs the right side.

\section{Computational Efficiency}

For the dispersion of dilute tracers, the standard BGK method can be simplified, and programs can be substantially optimized. A single lattice can be used for a carrier fluid, which determines a velocity field $\mathbf{u}$ obeying the Navier-Stokes equations; typically we use the 19-vector D3Q19 lattice (figure 1 ) with the $2^{\text {nd }}$-order equilibrium distribution given by Martys and Chen: ${ }^{7}$

$$
\begin{gathered}
f_{0}^{e q}=\frac{\rho}{3}\left[1-\frac{3}{2} u^{2}\right] \\
f_{t}^{e q}=t_{i} \rho\left[1+3 \mathbf{e}_{i} \cdot \mathbf{u}+\frac{3}{2}\left(3 \mathbf{e}_{i} \mathbf{e}_{i}: \mathbf{u u}-u^{2}\right)\right] \quad \text { for } i \neq 0 ;
\end{gathered}
$$

where $\rho=\sum_{i} f_{i}$, and $t_{i}=1 / 18$ for the 6 vectors along the Cartesian axes, and $t_{i}=1 / 36$ for the remaining vectors. The $\mathbf{u}$ determined from the carrier fluid is then used for each tracer. Flekkøy et $a l^{8}$ and Noble ${ }^{9}$ noted the advection-dispersion equation is linear in $\mathbf{u}$, so the tracer equilibrium distributions need only be linear in $\mathbf{u}$ as well. For a tracer $s$, the equilibrium distribution is of the form:

$$
f_{s, i}^{e q}=A+B\left(\mathbf{e}_{s, t} \cdot \mathbf{u}\right)
$$

and the $A$ and $B$ are fixed by the requirement that solute be conserved ( $\rho_{s}=\sum f_{s, i}^{e q}$ ), and the requirement that the solute flux at equilibrium is due entirely to advection $\left(\sum f_{\mathrm{s}, \mathrm{i}}^{\mathrm{eq}} \mathbf{e}_{s, l}=\rho_{s} \cdot \mathbf{u}\right)$. Compared to Eq. (3), Eq. (4) requires far fewer floating point operations. 


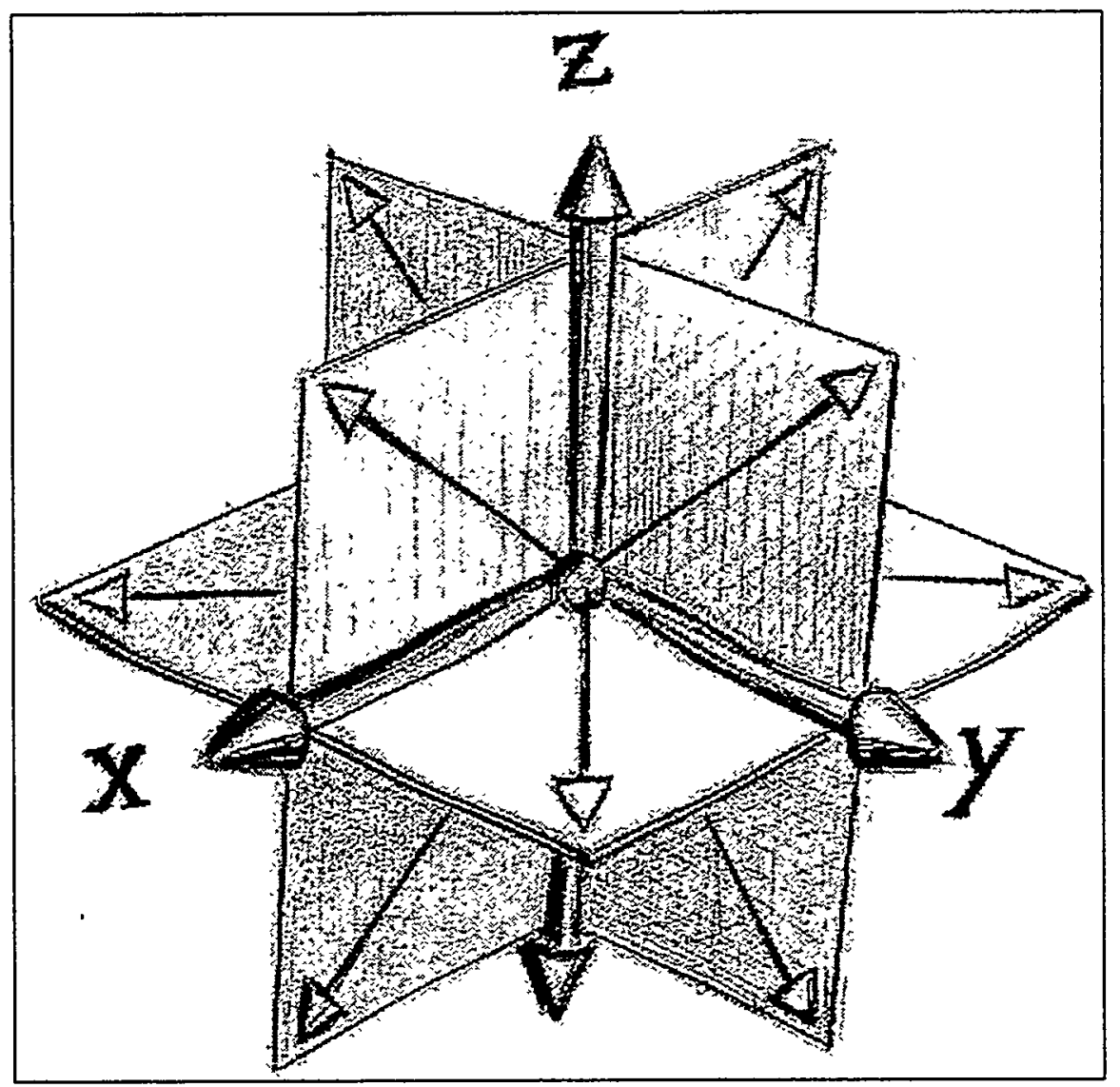

Figure 1. The 19 vectors of the D3Q19 model. There is a "rest" particle at $(0,0,0)$, there are 6 vectors with weight $1 / 18^{\text {th }}$ along the cartesian axes, and 12 vectors with weight $1 / 36^{\text {th }}$ in the $x-y, y-z$ and $x-z$ planes.

Further savings are realized by using lattices with fewer vectors for the tracers; in 2D, 4 Cartesian vectors are adequate, and in $3 \mathrm{D}$, the six Cartesian vectors will suffice. WolfGladrow $^{10}$ and others have suggested low-vector lattices for modeling diffusion, but Noble ${ }^{9}$ appears to have first performed a Chapman-Enskog expansion for Cartesian lattices, recovering the advection-dispersion equation and estimating the error term. (We emphasize that low-vector lattices, and Eq. (4), are used only for the dilute tracers, not the carrier.)

In many dispersion problems, $\mathbf{u}$ is at steady state, and need not be recalculated at each timestep. Furthermore, periodic boundary conditions on $\mathbf{u}$ are often appropriate, even when tracer dispersion is not periodic, as in the SC dispersion problem described in section 3. A strategy for such problems is to use a 19-vector carrier fluid in a single repeat unit, $N_{x}$ by $N_{y}$ by $N_{z}$ lattice units on a side, then save the equilibrium $\mathbf{u}(x, y, z)$. The memory used for the 19-vector carrier fluid is then reclaimed, and reallocated for additional repeat units of the tracer lattices in the $x$ direction. The velocity field at $x, y, z$ is then $\mathbf{u}\left(\left(x \operatorname{MOD} N_{x}\right), y, z\right)$.

The efficiency of the overall algorithm depends a great deal on the storage of the 3D lattice in memory. For systems that contain a high proportion of solids, it is often practical to store only those sites that represent open fluid or solids bounding fluid; a 3D pointer table obtains the location of the vectors in a linear array. Such an approach often leads to high locality, and can speed up the overall algorithm, by reducing the number of loads to the cache. For simplicity, we 
focus here on data structures that allocate the same amount of memory to all sites, be they solid or fluid. We next consider the tradeoffs inherent in data structures that optimize for the translation vs. collision step.

Let indices $s, i, z, y$, and $x$ represent, respectively, the chemical component, vector number (e.g. $i=0$ through 18 in D3Q19), and the Cartesian directions. Two obvious alternative schemes (for coding in C) are:

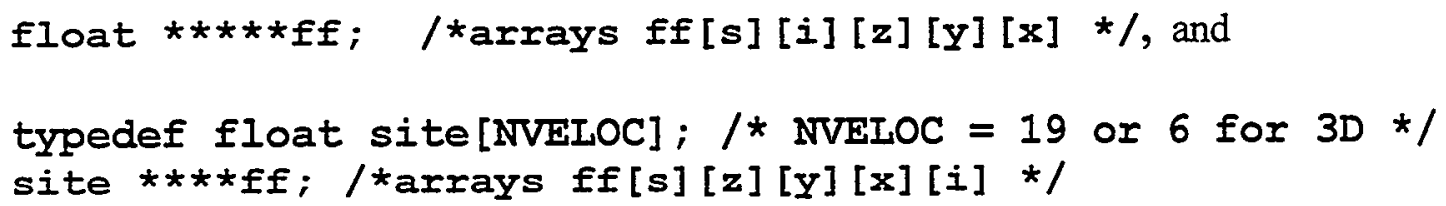

The latter holds all the vectors (for a given $x, y, z$ ) close in memory, and might seem much more efficient. However, the first method can make the translation step very fast, principally because much of the translation is accomplished by pointer swaps. The efficiency is most significant for 3D, 6-vector tracers; only two of the six vectors are actually moved in memory, and those moves (in the + and $-x$ directions) involve just one index and are easily optimized. The second method generates much cache traffic, and the translation step requires substantial effort to decompose and redistribute the vectors from each site. With all x86 compilers tested, the first method proved to be $\sim 1.05$ to 1.3 faster times than the second, and the discrepancy increased as the fraction of solids in the system increased.

Figure 2 gives simplified source code for a collision function involving just one 3D, 6-vector tracer and a predefined, static flow field uu. To lessen the burden of dereferencing 5dimensional arrays, the pointers are dereferenced manually in steps, at the beginning of the inner loops. To ease the alias problem that plagues the $C$ computer language, the 6 vectors for each site are copied into local $f 0, f 1, \ldots f 5$ for most manipulations. The MOD operation is not performed explicitly, as the latter would involve a floating point division, which would consume $\sim 20 \%$ of the CPU cycles. Finally, by factoring the $f^{e q}$ equations, the number of arithmetic operations is greatly reduced. With judicious use of register declarations, the most important pointers and local variables can be kept in registers throughout the calculation, even on an Intel x86 chip. 


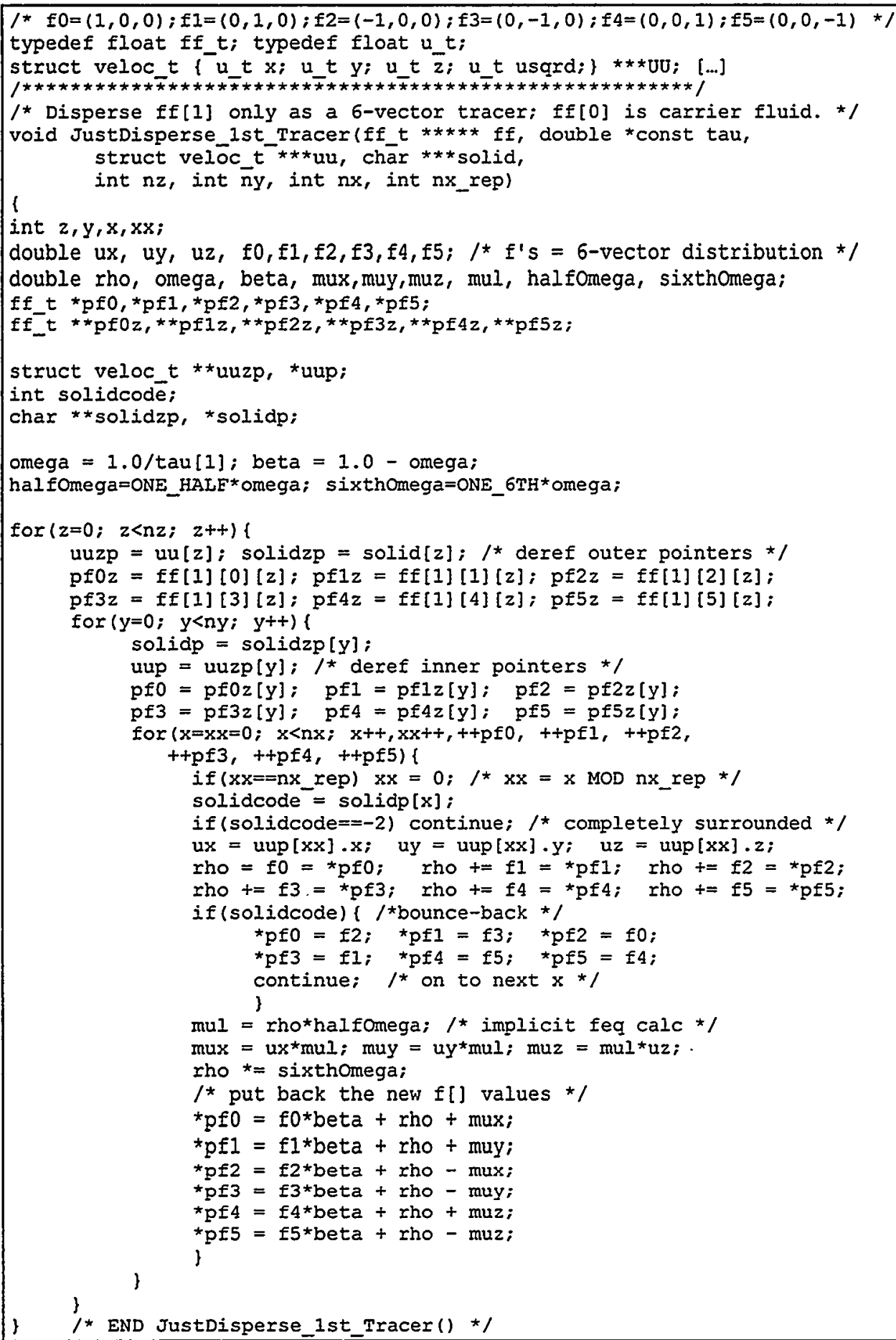

Figure 2. Simplified collision function for one 3D 6-vector tracer, $\partial u / \partial t=0$.

Table 1 shows performance achievable on single-processor CPUs for several applications, given in units of MUPs (millions of site updates per second). For comparison, rates of 0.14 to 2 MUPs have been reported ${ }^{9,11}$ for 2- and 3-D calculations on a 64-node partition of the CM-5, containing 256 vector processors. This comparison is not intended to denigrate parallel computers. We estimate the 6 -vector dispersion algorithms $\left(2^{\text {nd }}\right.$ test problem, table 1$)$ are nearing the RAM streaming limit, with speed controlled by the rate that site data can be fetched from and returned 
to memory. In such conditions, it makes more sense to split the workload among many processors, rather than seek a faster CPU.

Table 1. Performance for LB algorithms (MUPs = Millions site Updates Per Second).

\begin{tabular}{cc}
\hline Processor & MUPs \\
\hline Test problem: 19 -vector flow (no tracers), & 50\% solids \\
\hline 200 MHz Pentium Pro & 0.29 \\
$400 \mathrm{MHz}$ Pentium II & 0.70 \\
$500 \mathrm{MHz}$ alpha 21164a & 1.10 \\
Test problem: $3 D$ 6-vector tracer dispersion, steady flow field, \\
10\% solids & 1.55 \\
$200 \mathrm{MHz}$ Pentium Pro & 2.55 \\
$400 \mathrm{MHz}$ Pentium II & \\
Test problem: $3 D$. 3-component double-diffusive fingering with \\
buoyancy \\
200 MHz Pentium Pro & 0.15 \\
$400 \mathrm{MHz}$ Pentium II & 0.30 \\
\hline
\end{tabular}

The generation of "denormal" numbers can greatly degrade performance in LB dispersion calculations. Denormals are part of the IEEE 754 standard, and are used to provide gradual underflow, allowing one to represent numbers smaller than $\sim 10^{-37}$, the traditional lower limit of IEEE single precision. ${ }^{12}$ Most microprocessors do not handle denormals in hardware; instead, they provide the option of either ignoring the IEEE standard, and rounding the denormal to 0 , or they pass the value to a software routine for handling. Since the latter process can be excruciatingly slow, the compiler default is generally to round to 0 . However the Intel $x 86$ processors, such as the Pentium Pro, not only handle denormals in hardware, but provide no option to round these values to 0. Manipulations of denormals in x86 processors are slow, flushing the processor pipeline with each load. The problem can be delayed by using double precision, with a factor $\sim 1.6$ loss in initial speed and factor 2 loss of memory efficiency, and no gain in true accuracy for dispersion coefficients.

Slug dispersion problems have a strong tendency to generate denormal numbers. As a slug of solute travels downstream, very low concentrations are produced on the leading and trailing edges of the slug. In short order, the system contains many concentrations less than $10^{-37}$. Benchmarks with dispersion around cylinder and sphere arrays showed up to a factor 6 performance loss within $\sim 10^{4}$ steps. This weakness of denormal processing was recognized by William Kahan, the principal architect of the IEEE standard, and a simple solution is given in his Berkeley lecture series (available on the world-wide-web as ieee754.ps): all initial and boundary conditions that are formally at 0 concentration, are set instead to a small number, say $10^{-32}$. This artifice eliminates the slowdown, and has no effect on the calculated dispersion coefficients. 


\section{Accuracy}

\subsection{Permeability of a Simple Cubic Array of Spheres}

The permeability of a simple cubic (SC) array of spheres, at low $R e$, is well known ${ }^{13}$ and provides a basic benchmark for testing pixelation effects and the applicability of $L B$ to porous flow in single-component systems (i.e. systems that do not involve dispersion). The SC spheres are centered at the eight corners of a cube, as shown in figure 3 ; however, with wrap boundary conditions on opposing faces, a single sphere in a unit cube is adequate to model an infinite array. Pixelation refers to the representation of the sphere surface with a finite number of solid sites, so that the channels between the spheres appear somewhat jagged (figure 3). For nonoverlapped spheres, the permeability $k$ (units of length ${ }^{2}$ ) is related to the drag coefficient $C_{d}$ via:

$$
k=V /\left(6 \cdot \pi \cdot R \cdot C_{d}\right)
$$

where $V$ is the unit cell volume, and $R$ is the sphere radius (e.g., Saeger et al. ${ }^{14}$ ). For Darcy flow,

$$
k=-U \cdot \mu /(d P / d x)
$$

where $U$ is the superficial (or Darcy) fluid speed, averaged across an inlet or outlet face of the unit cell (including solids), $\mu$ is the dynamic viscosity, and $d P / d x$ is the pressure gradient along the $x$-direction. Formally, the LB used in the present study is a constant-pressure model, so the pressure gradient must be related to the body force. To relate the permeability to units measured in the LB simulation, we apply a force balance:

$$
x B o d y \cdot p \cdot X^{3}=\left(P_{\text {in }}-P_{\text {out }}\right) \cdot X^{2}=-(d P / d x) \cdot X^{3}
$$

or $(d P / d x)=-x B o d y \cdot \rho$, where $X$ is the side of the simulation cell (in lattice units), $x B o d y$ is the body force, applied in the $x$-direction, per unit volume in the LB simulation, and $\rho$ is the particle density per site specified in the LB simulation. Thus

$$
k=U \cdot v / x B o d y,
$$

where $v=\mu / \rho=(\tau-1 / 2) / 3$ is the kinematic viscosity specified in the LB simulation. Customarily one reports $k / X^{2}$ rather than $k$, to achieve scale-independence. 


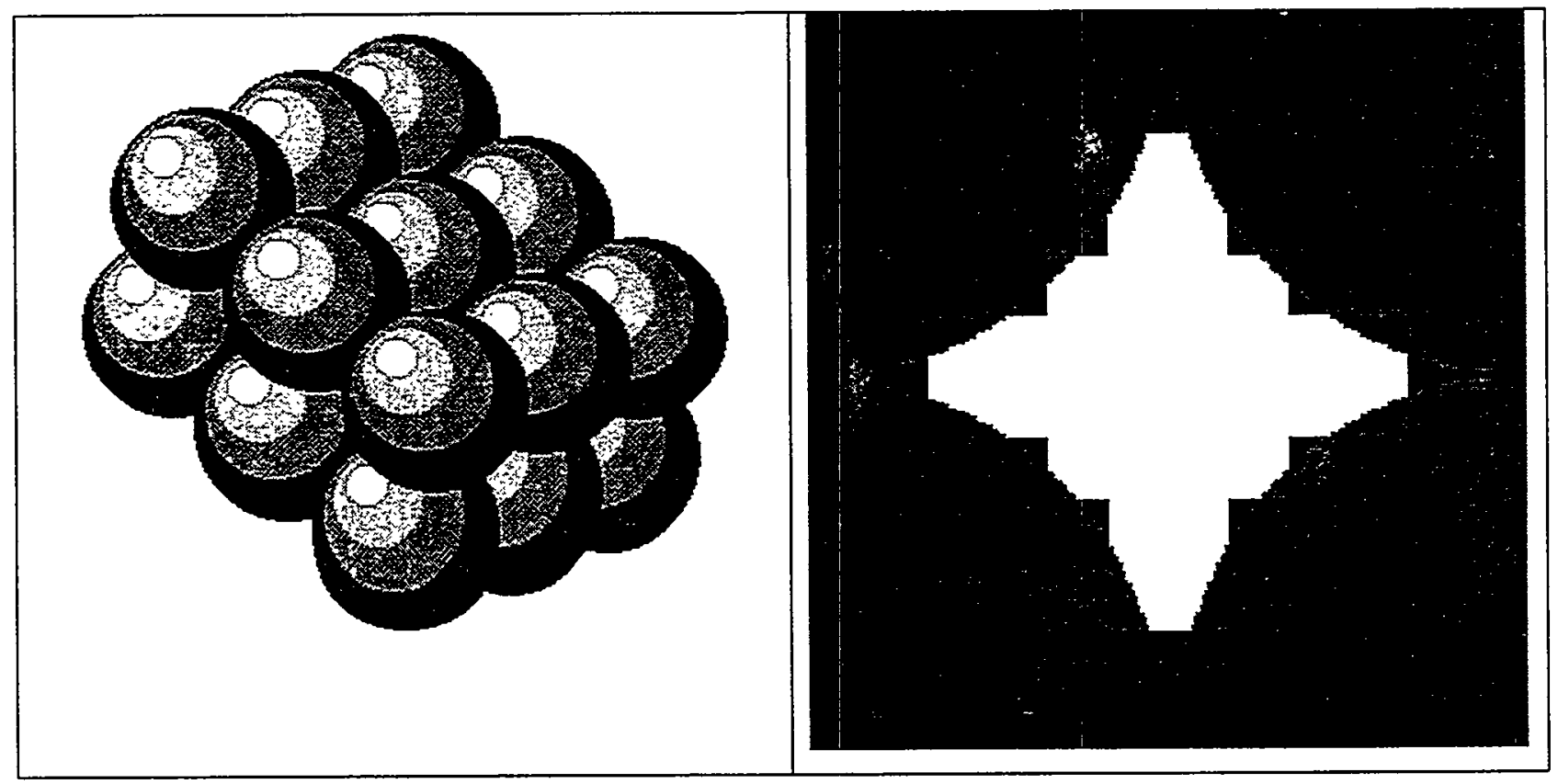

Figure 3. Simple Cubic (SC) geometry. At left is a small section of an array. Right, a face of the unit cube, showing the entrace to a channel; the "ideal" spherical sections are show in red, and the pixelation for $X=12(R=6)$ are shown by the black outline.

Figure 4 plots the apparent $k / X^{2}$, measured in the LB simulation at $R e=0.25$, as a function of $X=2 R$. The error in the measured permeability drops from $\sim 15 \%$ at $X=12$, to $\sim 1 \%$ at $X=36$. Most of the error at low $X$ can be attributed to pixelation effects; figure 3 compares the ideal geometry of the outlet channel, vs. the actual configuration for $X=12$. In the latter case, a greater fraction of the flow is in the center of the channel, lowering the apparent permeability. The oscillations in the LB permeability are probably a function of the algorithm used to "draw" spheres with integer radii within the unit cube. Figure 5 shows the normalized permeability for the case of non-touching spheres $(R=X / 3)$, along with the ratio of ideal (or target) sphere radius to the true, average radius used in the simulation. It is clear that the oscillations in LB-measured permeability correlate with the deviations of the sphere geometry from the ideal. 


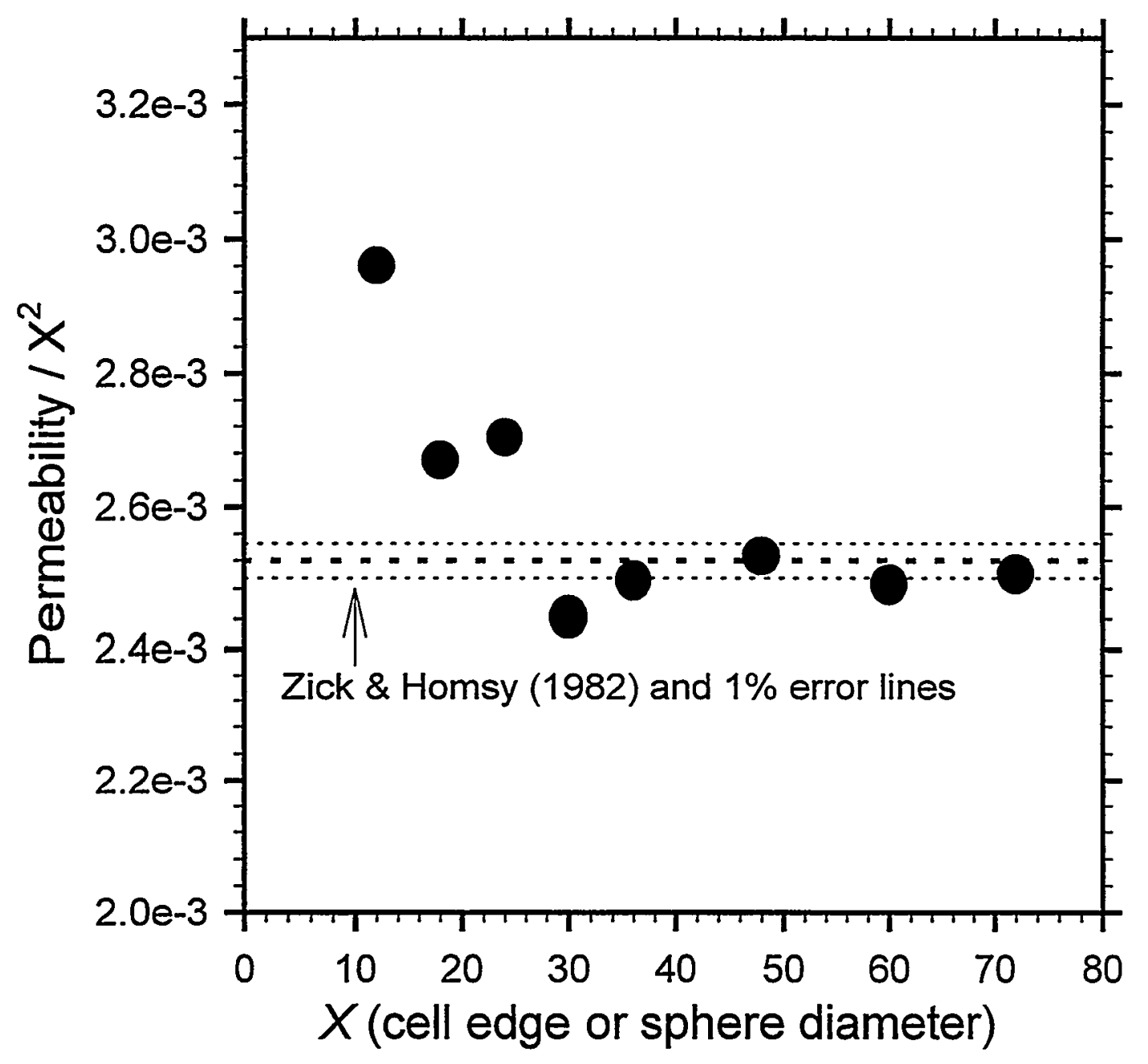

Figure 4. LB estimate of permeability of SC array of spheres, as function cell edge $X=2 R$. The deviation from the Zick and Homsy ${ }^{13}$ value is due mainly to the pixelation effect shown in figure 3. 


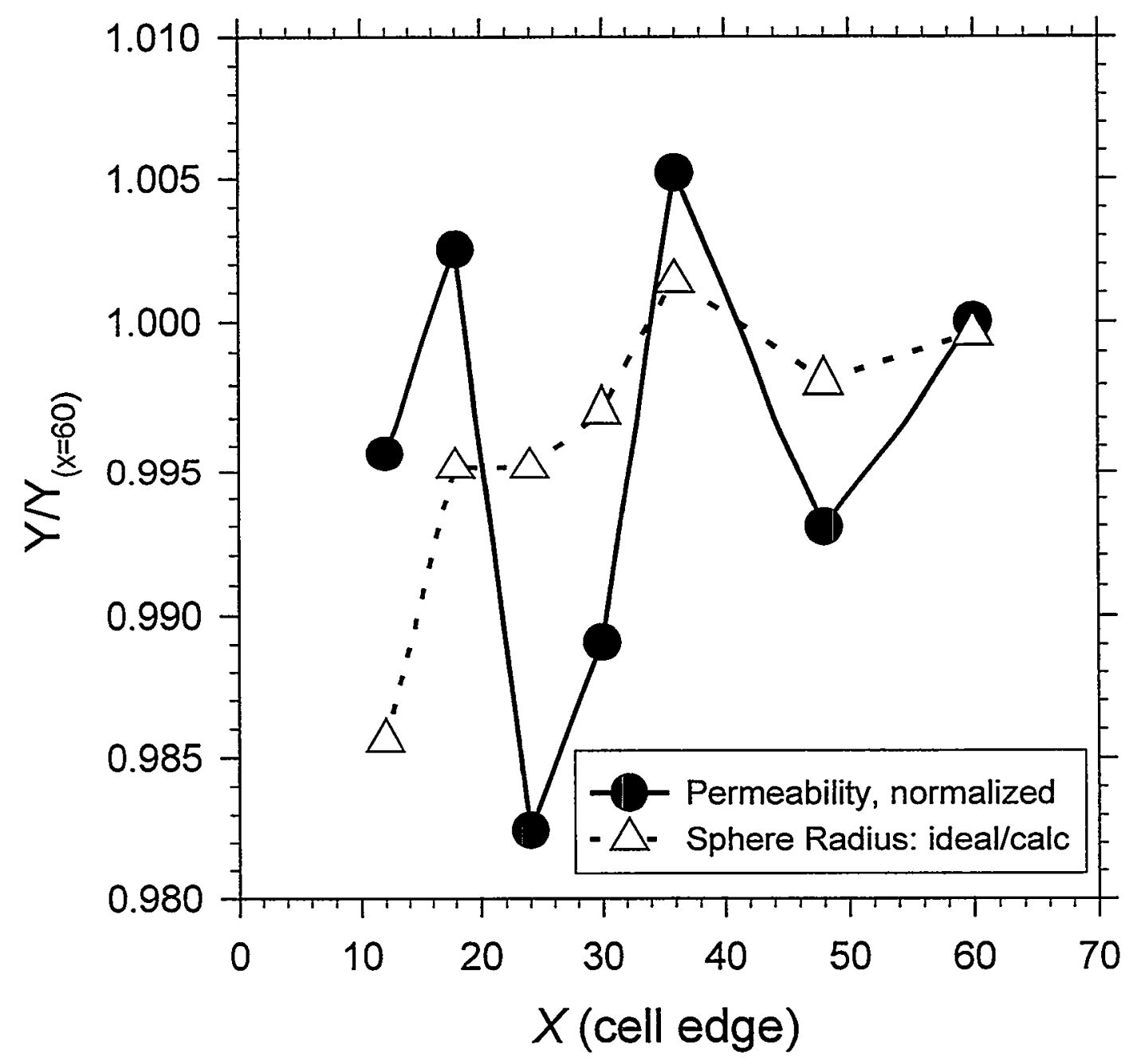

Figure 5. LB normalized permeability and sphere radii for the non-touching spheres, $R=X / 3$.

\subsection{Wall Conditions: Taylor-Aris Dispersion between Infinite Planar Sheets}

The Taylor-Aris problem for parallel plates is perhaps the simplest dispersion benchmark. The test geometry is shown on the left side of figure 6. A slug of solute is injected into an established, left-to-right Poiseuille flow between two parallel plates. Aris ${ }^{15}$ showed that after some characteristic time $t_{c} \sim h y^{2} / D_{m}$ (where $h y$ is the channel width and $D_{m}$ is the molecular diffusion coefficient), the projection of the solute distribution onto the $x$-axis is nearly Gaussian, and the dispersion coefficient $D^{*}$ follows the simple rule:

$$
D^{*} / D_{m}=1+P e^{2} / 210
$$


where the Péclet number $P e \equiv(h y \cdot U) / D_{m}$ ( $U$ is the cross-channel averaged speed). Equation (9) is taken as the predicted $D^{*} / D_{m}$. We can also "measure" $D *$ for our numerical experiment by the method of moments: ${ }^{15}$

$$
D^{*} / D_{m}=1 / 2 d m_{2} / d t / D_{m}
$$

where $m_{2}$ is the second moment, or variance $\left(\sigma^{2}\right)$ of the solute distribution, projected onto the $x$ axis. The difference between equations (9) and (10) is our estimate of error in figure 6 (calculated for a fixed $P e=20$ ). The figure compares results for two different wall collision methods. The distinction between these two methods is described below.

In recent years, much was written on the choice of wall conditions for LB calculations. The basic problem is that after the streaming step, there are $f_{i}$ (equation 1 ) pointing into the solids, but none pointing out from the solids; these missing particle distributions must be created in a consistent manner. For pure flow calculations, the simple bounce-back condition, which fills in the missing $f_{i}$ by inverting particle distributions at the solid wall, can yield acceptable accuracy. To achieve such accuracy with bounce-back, the collision parameter $\tau$ is kept reasonably low, and the wall solid wall is taken to be $1 / 2$ lattice unit out from the solid nodes. ${ }^{16}$ The subterfuge of using the $1 / 2$ location is commonly referred to as "reinterpreted" bounce-back. However, when applied to tracer particles, the justification for re-interpreted bounce-back is less clear, since the tracers have some residence time "behind" the $1 / 2$ wall.

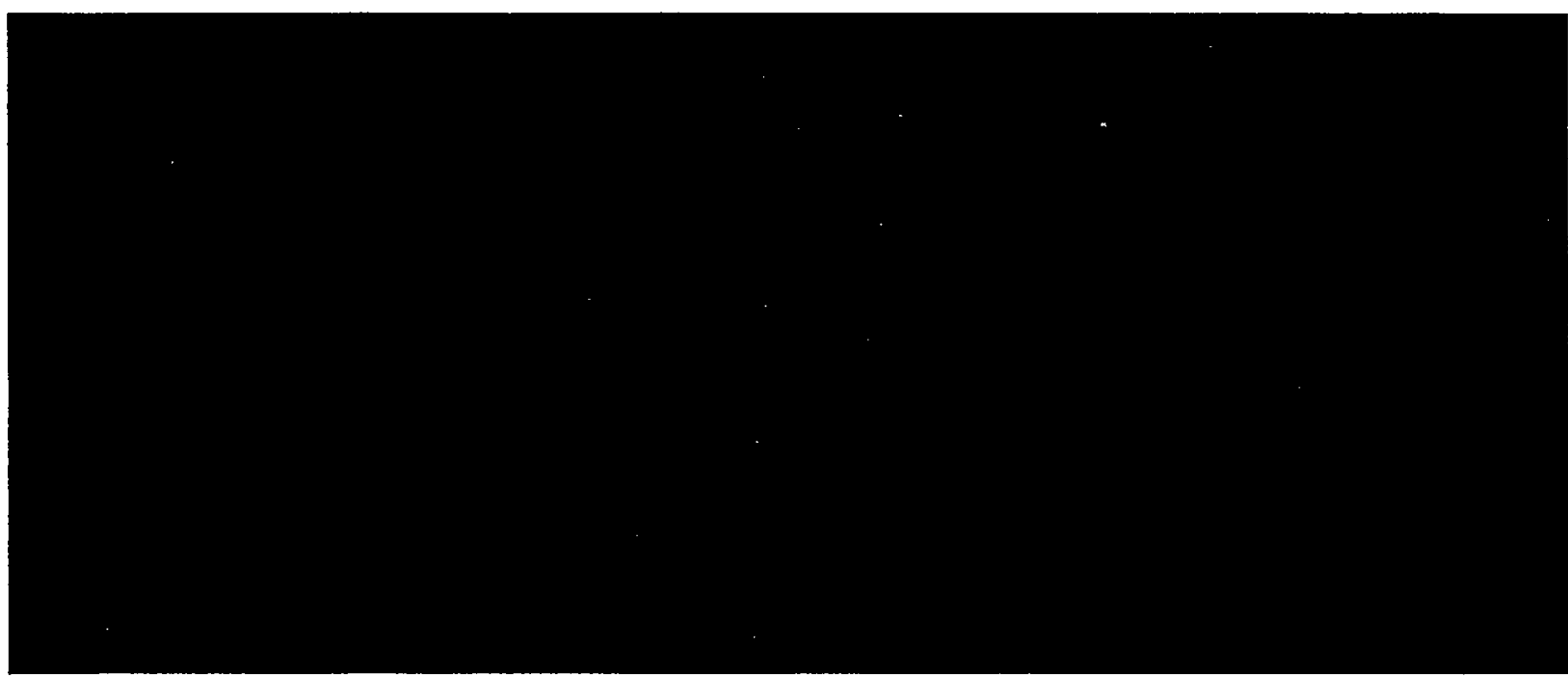

Figure 6. Taylor-Aris dispersion between infinite parallel plates. The test geometry is at left; a slug of solute is injected into an established, left-to-right Poiseiulle flow. As the slug spreads, the projection of concentration onto the $x$-axis eventually becomes Gaussian. At right is the error for two types of wall conditions; traditional bounceback and a no-flux condition (error is difference between equations 9 and 10).

An alternative wall condition for tracer dispersion was suggested by Noble. ${ }^{9}$ In brief, one places the zero-flow position on the wall, by one of the many methods suggested in the literature. ${ }^{16,17}$ Both carrier fluid and tracer particles are allowed to stream along the walls. For tracer particles, the missing part of the distribution is obtained by reflecting across a plane tangent to the wall; in 
effect, this is a slip condition. The tracer distributions then are sent through the relaxation step, in which they inherit the diffusion coefficients typical of the free fluid. For all but the narrowest channels, the method suggested by Noble (figure 6) proves more accurate than the simple bounce-back. The bounce-back method reaches a peak error of about $3.8 \%$, before slowly decreasing. The causes of this non-monotone behavior are not clear, but there are probably several competing effects.

Despite the modest accuracy, there are several good reasons to retain bounce-back for dispersion problems. The first is that the method leads to extremely simple and efficient code. Second, with bounce-back, there is a simple relationship between the number of solid nodes in the system and the volume of solids modeled; the benefits of this simple relationship become more obvious when one attempts to implement dissolution and precipitation, which can create very complicated patterns of dendritic growth. If growth occurs by a transition state rule, ${ }^{18}$ the surface area and volume of each point in the automaton must be consistently known. Third, the use of bounce-back can be justified on a simpler basis: dispersion data in experiments are rarely measured to an accuracy of better than $10 \%$, and theories for predicting dispersion in complex, $3 \mathrm{D}$ geometries rarely come within a factor of 2 of experimental measurements. Consequently, bounce-back walls were used in the following two benchmark exercises.

\subsection{Taylor Dispersion in a Duct with Rectangular Cross-Section}

Consider the 3D duct shown in figure 7 , which extends infinitely along the $x$-axis, but is closed on the other four walls. It is commonly assumed that if $h y / h z<<1$, dispersion in the duct will follow the Taylor-Aris solution (Eq. 5). Surprisingly, as long as the duct has vertical sidewalls, dispersion never approaches the Taylor-Aris solution, regardless of the aspect ratio. As $h y / h z$ $\rightarrow 0$, the solution approaches:

$$
D^{*} / D_{m}=1+7.95 P e^{2} / 210
$$

so for high $P e$, the close-walled duct approaches a dispersion coefficient nearly 8 times theTaylor-Aris value. Figure 7 gives a hint about the origin of this enhanced dispersion; the edges of the solute slug are swept back by the vertical duct walls, since the flow speed must vanish at the walls. 


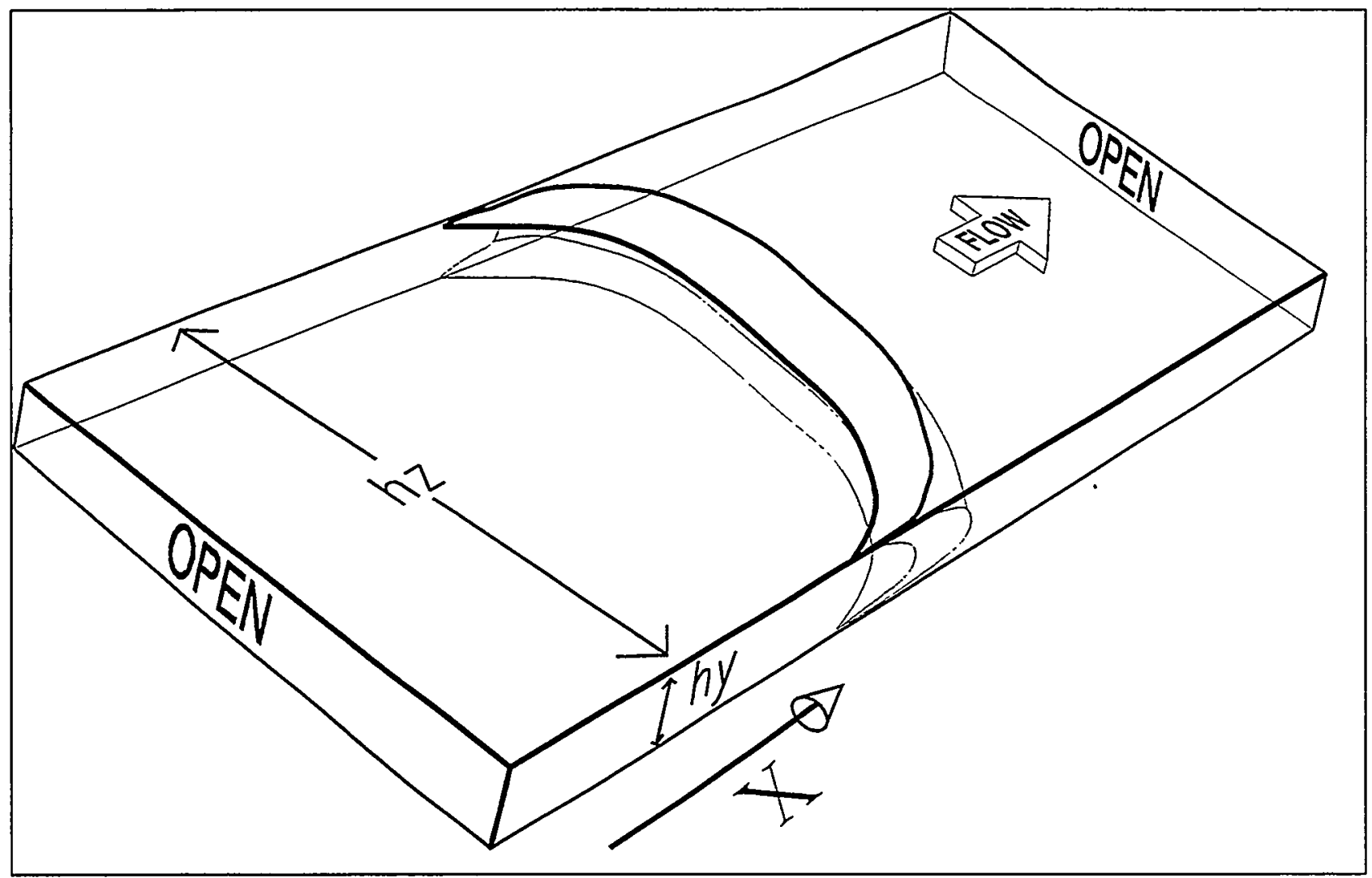

Figure 7. Geometry for dispersion in a rectangular duct.

The time required to reach a dispersive steady state in the duct is $t_{D}=c \cdot h z^{2} / D_{m}$, where the proportionality constant $c$ is $\leq 1 / 2$. Thus the duct must be of length $h x \sim c \cdot U_{x} \cdot h z^{2} / D_{m}$, where $U_{x}$ is the average flow speed in the $x$-direction, to contain the slug on its path toward the outlet. We define the Péclet number relative to the narrow dimension, $h y$, as $P e_{y}=U_{x} \cdot h y / D_{m}$, and the aspect ratio $n=h z / h y$. In general, the DDG problem is studied for a variety of $n \rightarrow \infty$, for as high a $P e_{y}$ as is possible. The total number of sites required for the simulation is $h x \cdot h y \cdot h z \sim c \cdot P e_{y} \cdot h^{3} \cdot h y^{3}$; the total clock time required for a run $\propto t_{D}$ (number of sites)/(MUPs.10 $\left.10^{6}\right)=$ $c^{2} \cdot P e_{y} \cdot n^{5} \cdot h y^{5} / D_{m} /\left(\right.$ MUPs $\left.\cdot 10^{6}\right)$. Thus, for $h y=7$ and $n=7$ and $P e_{y}=14$ achieved by $U_{x}=0.03$ and $D_{m}=0.015$, we require $\sim 0.83$ million sites, and at $2.55 \mathrm{MUPs}$, the calculation takes $\sim 7$ hours. While this amount of time is entirely feasible on a desktop computer, it provides motivation for more efficient methods that do not grid the lattice uniformly. ${ }^{19}$

Figure 8 compares our measurements of the DDG effect with the calculations of Chatwin and Sullivan. ${ }^{20}$ The agreement is reasonably good, given that Chatwin and Sullivan used two methods that sometimes disagreed by $5 \%$. 


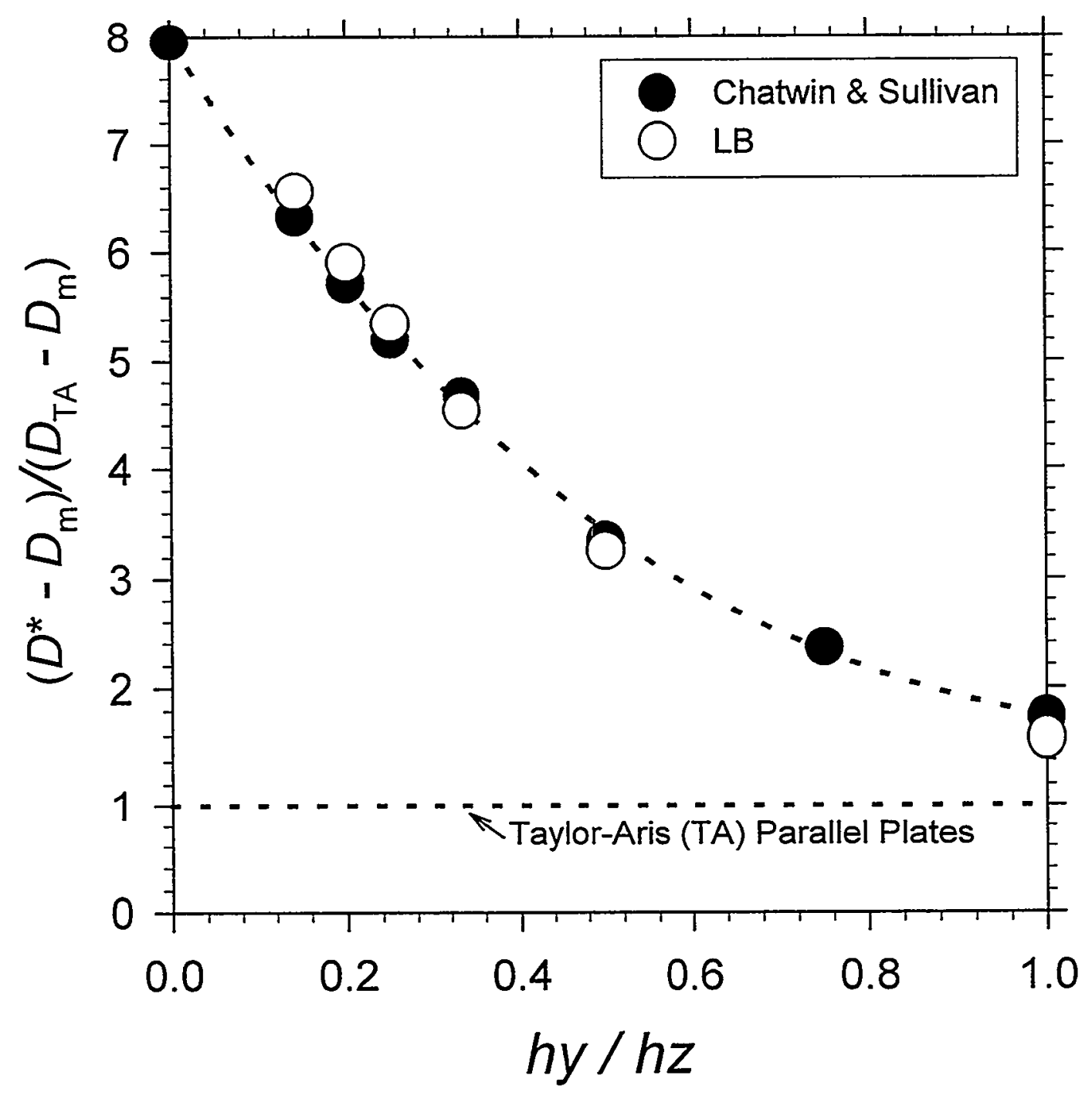

Figure 8. Comparison of LB calculations for dispersion in a rectangular duct, against the predictions of Chatwin and Sullivan. ${ }^{20}$

\subsection{Dispersion in an Infinite Array of SC Spheres}

Dispersion of tracers in porous media is of great interest in chemical engineering, where packed beds are used to effect chromatographic separations, or the solids act as catalysts for chemical reaction. In environmental chemistry and hydrology, there is a great interest in measuring the sorption of tracers in flowing experiments, with eventual application to field conditions. However, in laboratory-scale tests, it is often extremely difficult to distinguish sorption from dispersion, since both phenomena create similar tail effects. ${ }^{3,21,22}$ It is thus useful to have accurate benchmarks for dispersion in porous media analogous to packed beds, so dispersive 
effects can be quantified with reasonable accuracy. Since regular arrays provide the most reproducible and well-defined geometries, they are ideal candidates for benchmark exercises.

Surprisingly, there is little agreement among experimental results, and analytical or numerical solutions, for dispersion in regular arrays. Gunn and $\mathrm{Pryce}^{23}$ measured the dispersivity for argon gas in simple cubic (SC) and rhombohedral packings, using frequency response techniques. Subsequently, Eidsath et al..${ }^{24}$ Koch et al.,${ }^{25}$ Salles et al.,${ }^{26}$ and Lee et al. ${ }^{27}$ modeled these experiments, with limited success. Typically the experimental and modeled coefficients differ by factors of 2 to 7 , at particle Péclet numbers $(P e)$ from 1 to 300 . However, some assumptions made in the models are not wholly consistent with the experiments. For example, the Gunn and Pryce ${ }^{23}$ study achieved a Schmidt number $S c$ of $0.88,(S c \equiv P e / R e)$ and Reynolds numbers $(R e)$ up to 400 ; in contrast, the Koch $e t$ al. $^{25}$ and Lee $e t$ al. ${ }^{27}$ models implicitly assumed Stokesian flow, with $S c \rightarrow \infty$, and the Eidsath et al. ${ }^{24}$ models used $R e$ of 0.006 and 56. Kaviany ${ }^{28}$ and Salles et al. $^{26}$ identified the $R e$ difference as a potential cause of disagreement, but Kaviany also speculated the $\boldsymbol{R} e$ effect might be masked. The calculations by Eidsath et $a .^{24}$ approximated the spheres with 2-dimensional cylinder arrays with porosity $\varepsilon \sim 0.37$, compared to the actual SC value of $\varepsilon \sim 0.48$. The Koch et al. ${ }^{25}$ theory is strictly valid for $\varepsilon \sim 1$ only, assumes the solute is conducted through the solids, and does not completely satisfy the boundary conditions on the particle surfaces; Koch et al. speculated that the latter problem caused their model to underestimate dispersion.

For the present study, dispersion in an SC array was modeled with the LB method. The main goals of the simulation were (1) to determine if inertial effects (i.e. non-Stokesian flow) could be the source of disagreement between experiments and prior analyses, and (2) to provide a dispersion benchmark more complex than simple flow in a rectangular duct, particularly a benchmark capable of capturing tailing effects. Most of the calculations employed a 19-vector Navier-Stokes carrier fluid (as described in section 2), and a single 6-vector tracer. However, a single run was made with a 19-vector tracer for comparison purposes, at $\boldsymbol{R} \boldsymbol{e} \sim 150$. The 19vector and 6 -vector simulations produced dispersion coefficients that agreed within $0.2 \%$, so the 6-vector tracers (which run 3 times faster) were used for the bulk of the calculations.

As in sections 3.2 and 3.3, dispersion coefficients were measured by the method of moments. A slug of solute was placed at the upstream end of the array of spheres. The slug deforms and broadens as it travels down the column, and at steady state, the dispersion coefficient $D^{*}=1 / 2 d m_{2} / d t$, where the second moment $m_{2}=\sigma^{2}$, and $\sigma$ is the standard deviation of the solute distribution along the flow direction. The characteristic time to achieve steady state is $t_{c}=\alpha R^{2} / D_{m}$, where $R$ is the sphere radius. At steady state, $m_{2}$ increases linearly with time, at which point $D^{*}$ is truly constant and well-defined. The magnitude of $\alpha$ is not well established; Eidsath et al $^{24}$ report $0.36 \leq \alpha \leq 0.8$. In the present study, $m_{2}(t)$ was linear (e.g. correlation coefficient $r^{2} \approx 0.99999$ for a first-order fit) for $t \geq 1 / 4 R^{2} / D_{m}$; for the cylindrical geometry, a longer $t$ was required, because this geometry has inherently lower communication between the stagnant pockets and central channel.

Because of the wrap condition, the steady-state flow field $\left(u_{x}, u_{y}, u_{z}\right)$ need be calculated in only one cubic unit cell, containing a single sphere. Based on the results of section 3.1, a sphere of diameter $d=36$ in a cubic cell with edge $X=36$ was chosen, since this configuration yields a permeability accurate to $\sim 1 \%$. For the dispersive part of the problem, a column of spheres is necessary. As with the duct dispersion problem, the length of the column is set by the 
requirement that the slug stay within the column for $t \sim 1 / 4 r^{2} / D_{m}$; for the highest $P e$ studied, this requirement translates to a column of $\sim 60$ spheres.

Figure 9 illustrates the flow fields for the $\boldsymbol{S c}=0.88$ and $\boldsymbol{S c}=32$ cases $\left(u_{x}, u_{y}\right.$ components alone are shown; the $z$ axis points into the page). At the lower $S c$ (higher Reynolds number), there are clear recirculation zones in the lee of the sphere. Compared to the $\boldsymbol{S c}=32$ case, the $\boldsymbol{S c}=0.88$ case has a higher flow rate in the top and bottom regions, given the same Darcy velocity. 


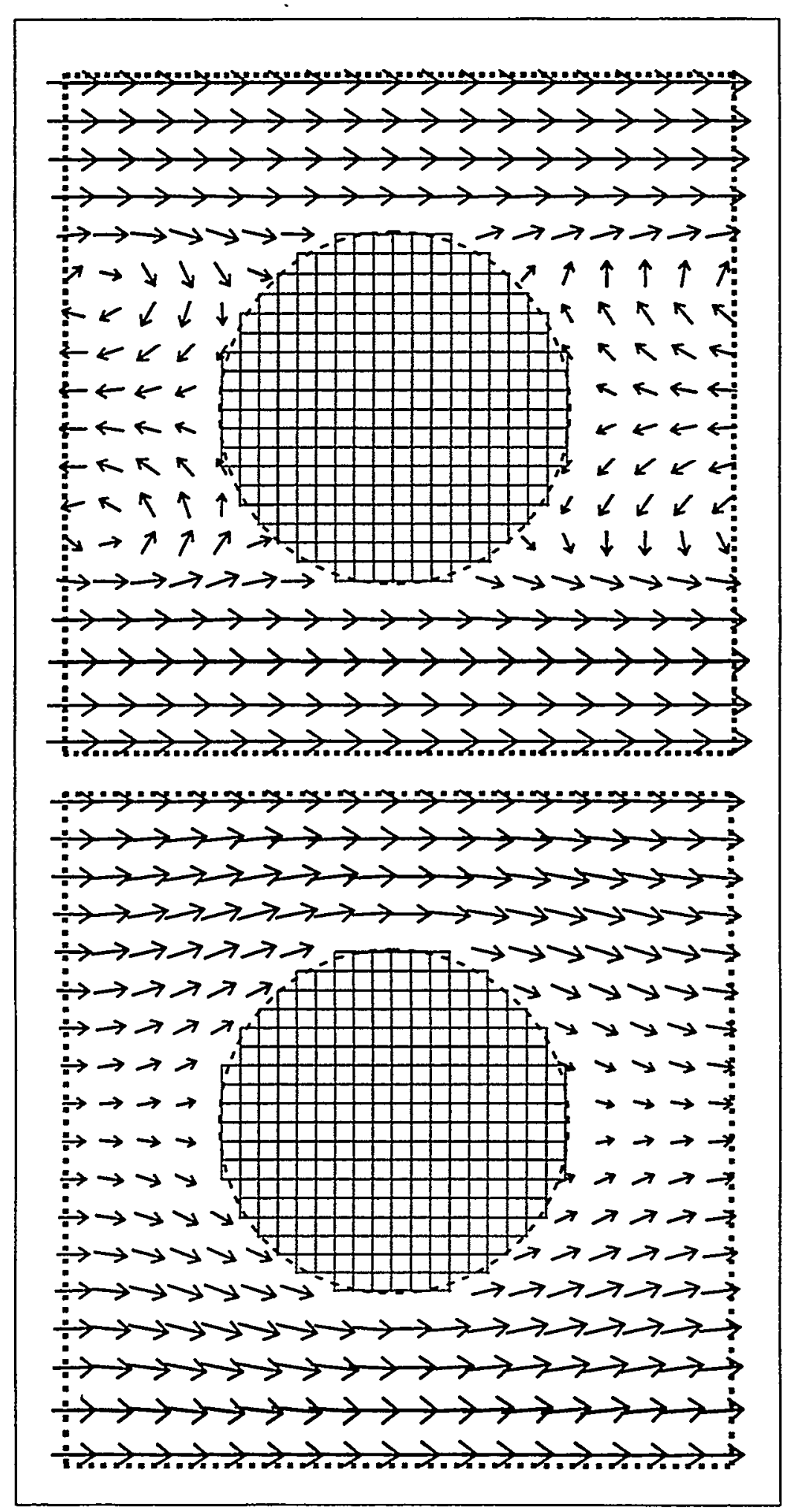

Figure 9. LB flow fields for $R e=150$ (top) and $R e=4.1$ (bottom), for the plane at fractional depth $z=0.0694$ (2.5 lattice units). For $P e=132$, the corresponding $S c$ are 0.88 (top) and 32 (bottom). Vector lengths are $4^{\text {th }}$-root scaled to emphasize regions of low-speed flow. The true distribution of solids is indicated by the cross-hatch pattern; the dashed circle is the ideal spherical section for this $z$ position. 
The patterns of solute are shown, for early time $\left(t / t_{c}<0.3\right)$ and $P e=132$, in figure 10 . For the $S c=0.88$ case (top 4 frames), the solute is more effectively dispersed than in the $S c=32$ case (lower 4 frames). The enhanced dispersion appears to be caused by two effects: (1) greater average fluid speed in the channels between the spheres, at the same Darcy speed; and (2) residence and mixing of solute in the recirculation zones behind the spheres.

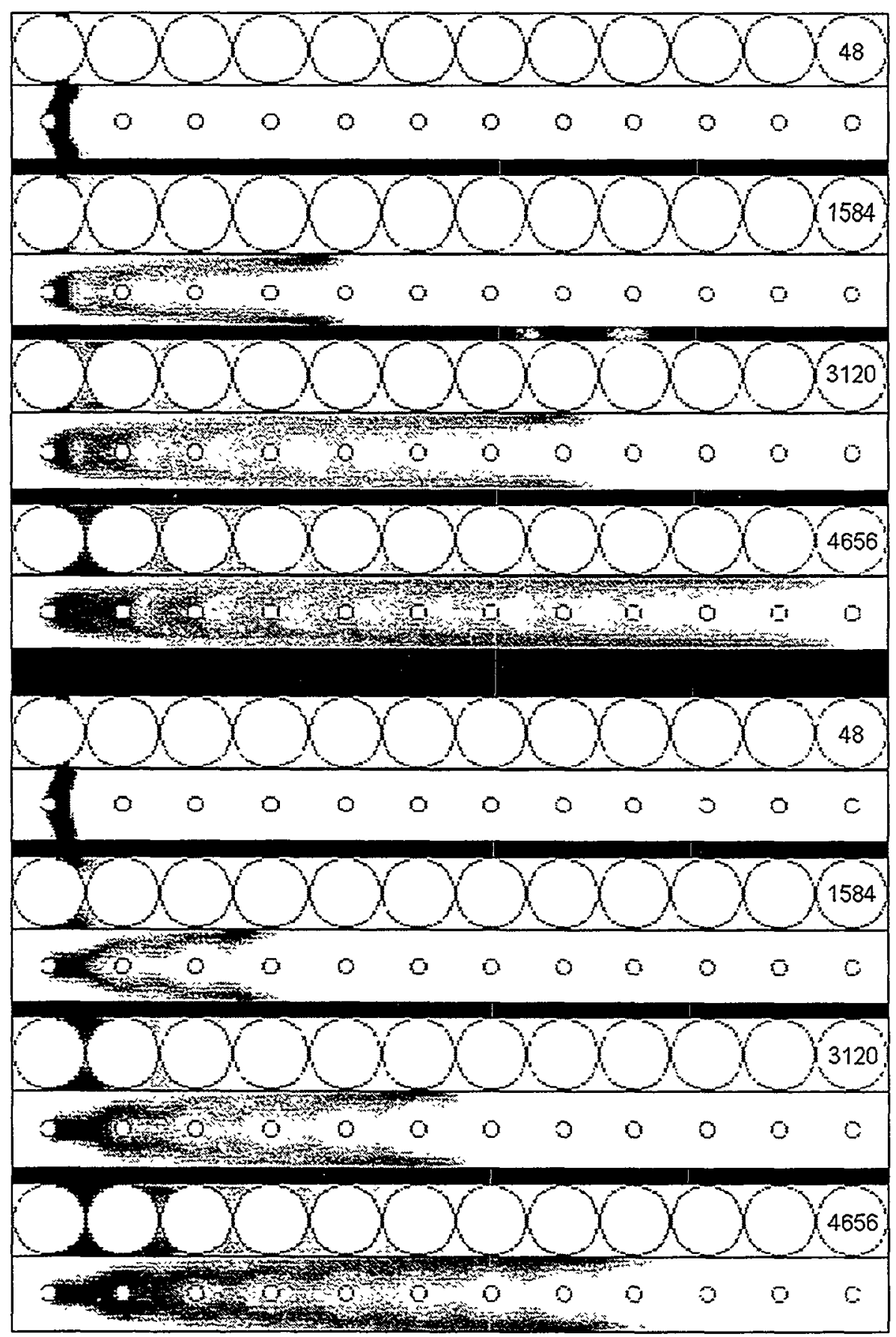

Figure 10. Solute patterns in the early stages $\left(t / t_{c}<0.3\right.$ ) of the LB calculations, for $S c=0.88$ (top) and $S c=32$ (bottom); both are at $P e=132$. The step number is indicated on the upper right of each frame. Each frame shows cuts at fractional depths $z=0$ and $z=1 / 2(0$ and 18 lattice units, respectively). The solute is indicated in black; intensities are square-root scaled to emphasize lower concentrations. The low-concentration ripples in the $t=48$ frames are numerical artifacts that are quickly damped. 
Figure 11 plots the LB-calculated dispersion coefficients against the $P_{e}=U d /\left(D_{m}(1-\varepsilon)\right)$, and compares our results against available experimental data, ${ }^{23}$ the Koch et al. ${ }^{25}$ theory for Stokesian flow past SC spheres, and the Eidsath et al. theory ${ }^{24}$ for flow past 2D cylinders. For the cylindrical geometry, the "effective" particle diameter $d_{p}=1.5 d$ is used, to allow direct comparison with the Eidsath et al. results. Note that the LB cylinder calculations match, within $\sim 10 \%$, the Eidsath et al. finite element results for this geometry. Thus there is some confidence that any differences, between the LB SC sphere results and the Eidsath et al. cylinder study, are not merely due to differences in numerical methods. For the SC sphere array, the LB $S c=0.88$ runs are quite close to the trend of the experimental data. In contrast, the $L B S c=32$ runs are between the Eidsath $e t$ al. and Koch $e t$ al. numerical results. From these comparisons, it seems reasonable to suggest: (1) the difference in $S c$ (and $R e$ ), including the presence of recirculation zones, accounts for some of the discrepancy between the Koch et al. theory and the gas dispersion experiments; and (2) the cylindrical geometry and $\boldsymbol{S c}$ assumed by Eidsath $e$ t $a l$. account for much of their disagreement with the experimental results. Note that with $\varepsilon=0.373$ in the cylindrical geometry, small variations in $\varepsilon$ can cause quite large changes in the measured transport coefficients (e.g. at $\varepsilon=0.215$, there are no gaps between the cylinders). Indeed, Eidsath et al. do not give a clear motivation for choosing $\varepsilon=0.373$, and they also show that rather small variations in the cylindrical geometry affect the measured dispersion coefficient by a factor of two or more. Overall, the LB results suggest the Gunn and Pryce experiments were reasonably accurate and representative for $\boldsymbol{S c}=0.88$. 


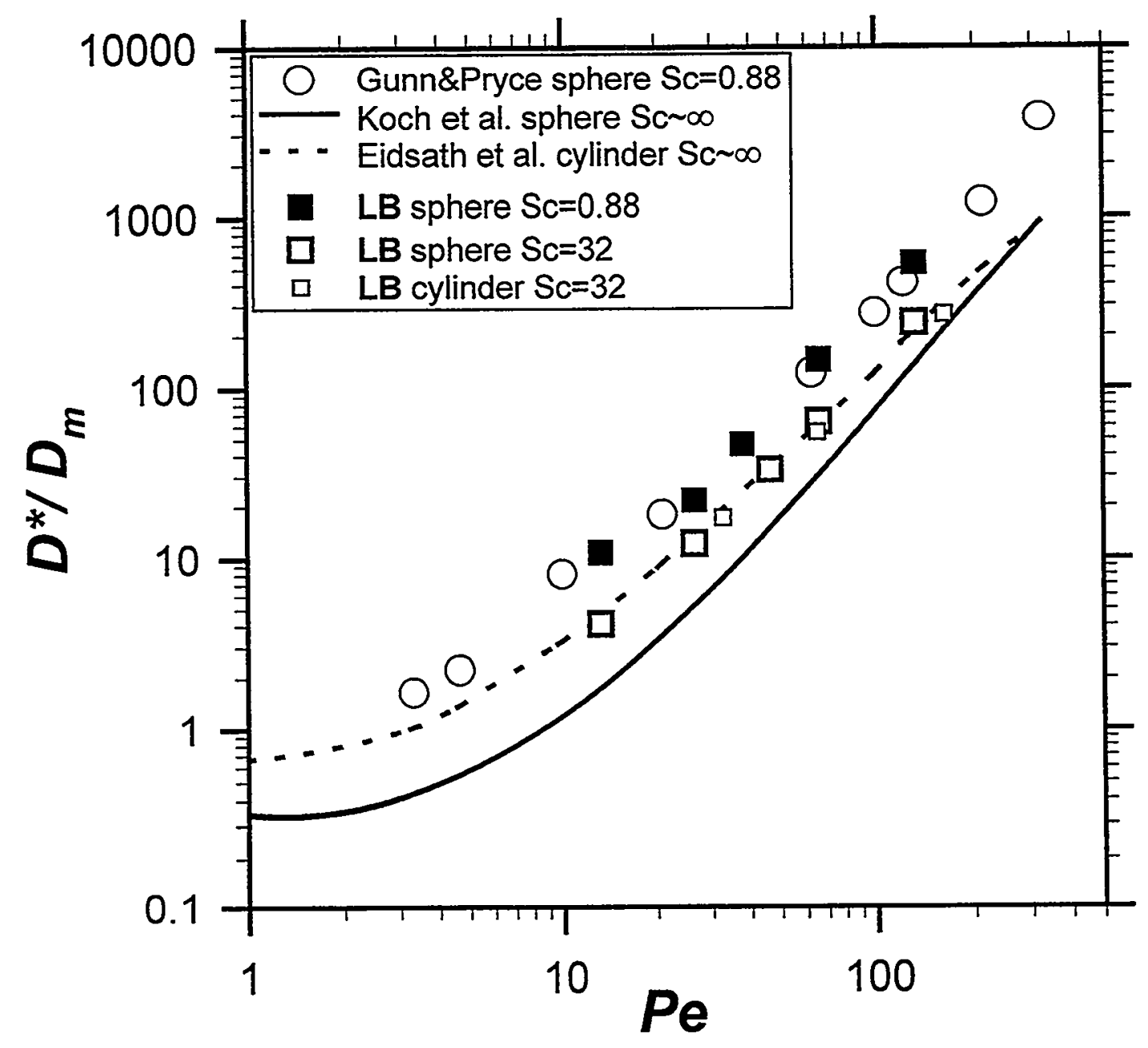

Figure 11. Comparison of LB results with experiments and theory. Gunn and Pryce ${ }^{23}$ points (open circles) represent gas dispersion experiments. 


\section{Code Architecture and Function Descriptions}

This section describes the basic JB_PH.C source for multi-component, 3D LB. Many variations on the basic code exist, better suited for problems involving fracture flow and double-diffusive convection. However, the streaming, collision, gravity-control and I/O algorithms are the same in most versions of the LB codes.

Throughout the listings, ncol, nrow and nlay (or the uppercase versions of these) refer to the sizes of the automaton in the $x, y$ and $z$ directions, respectively; that is, to the numbers of columns, rows and layers. The nveloc [s] refers to the number of velocity vectors for the LB lattice of component $\mathbf{s}$ (19 for the D3Q19 carrier, and 6 or 19 for the tracers), and ncomponent refers to the number of components in the system (always at least 1 , including the carrier).

\subsection{Compiling the Codes}

The codes described herein were all compiled with the Intel Reference Compiler Version 2.4, P97176, hosted from the Microsoft MS C++ 4.0 integrated development environment.

Benchmarks, run as part of this project, showed that the Intel compiler produced LB executables that were at least $30 \%$ faster than those produced by the Microsoft compiler, 3 times faster than the executables produced by Symantec C++ version 6.0, and 4 times faster than those produced by Watcom $\mathrm{C}++9.5$, using all recommended switches for code optimization. The switches used for the Intel compiler were -G6 -Qmem -Qip. The first switch optimizes for the Pentium Pro (and above) processors; the second optimizes memory loads and stores; and the third performs interprocedural optimizations, particularly in-line function expansions. It is notable that the Microsoft compiler produced fast executables only when anti-aliasing pragmas were used to encapsulate the collision functions, but the Intel compiler did not require such non-standard directives.

\subsection{JB_PH Command-Line Switches}

Many aspects of an LB calculation can be passed to JB_PH via command-line switches, which eliminate the need to recompile the code, between runs, simply to make minor adjustments to program size. The available switches can be determined by running the program with only the $-h$ or -? switches; this invocation will list the available switches on the console, and will then terminate the program. The listing will appear as: 


\begin{tabular}{|c|c|c|c|}
\hline FIAAG & ARGUMENT & WEAT IT SETS & IMPLEMENTED? \\
\hline--- & $--\infty--$ & $---n--m---$ & $----n$ \\
\hline$-\mathbf{x}$ & float & XBOdY, X BODY force & $\mathbf{y}$ \\
\hline$-\mathbf{y}$ & float & YBOdY, Y BODY force & $\mathbf{Y}$ \\
\hline$-u$ & float & uxIimit, $\mathbf{x}$ speed limit & $\mathbf{y}$ \\
\hline$-v$ & float & uyLimit, $y$ speed limit & $\mathbf{y}$ \\
\hline$-n$ & float & viscosity component 0 (carrier) & $\mathbf{y}$ \\
\hline$-a$ & float & diffusion coeff. component 1 & $\mathbf{Y}$ \\
\hline$-D$ & Eloat & Diffusion coeff. component 2 & $\mathbf{y}$ \\
\hline$-\mathbf{s}$ & integer & MAXSTEPS, maximum number of steps & $\mathbf{Y}$ \\
\hline$-j$ & integer & JUST_DISPERSE_STEP (flow frozen in) & $\mathbf{Y}$ \\
\hline$-c$ & integer & NCOI, number of columns ( $\max x)$ & $\mathbf{y}$ \\
\hline$-\mathbf{r}$ & integer & NROW, number of rows (max $y$ ) & $\mathbf{y}$ \\
\hline-1 & integer & NLAY, number of layers $(\max z)$ & $\mathbf{y}$ \\
\hline$-i$ & integer & AVELOC, \# velocity vectors for solutes & $\boldsymbol{y}$ \\
\hline$-b$ & integer & Boundary: $0=$ bounceback, $1=$ Ziegler & $\mathbf{n}$ \\
\hline$-g$ & integer & stepstartslug (step tracer slug) & $\mathbf{y}$ \\
\hline$-\mathbf{E}$ & string & filename containing solids geometry & $\mathbf{N}$ \\
\hline-0 & integer & Sphere Radius (for JO.C, JO_SPH.C) & $\boldsymbol{y}$ \\
\hline$-t$ & integer & Times (NTIMES) to multiply geometry & $\mathbf{y}$ \\
\hline 一\# & integer & $x$ and $z$ size alveoli grid (fin to fin) & $\mathbf{Y}$ \\
\hline$-\wedge$ & integer & Y height alveoli grid (inside measure) & $\mathbf{y}$ \\
\hline$-h \mid-?$ & <none> & display this list & $y$ \\
\hline
\end{tabular}

For example, if the program were invoked as:

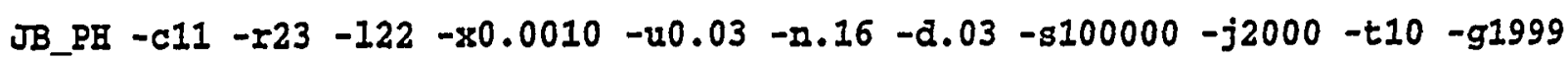

memory would be allocated, initially, for 11 columns (-c11), 23 rows (-r23), and 22 layers $(-122)$, corresponding to $x-y-z$ dimensions of $11 \times 23 \times 22$. A body force of 0.0010 would be applied initially in the $x$-direction ( $-\times 0.0010)$, until the $x$-direction speed across the outlet measuring plane reached 0.03 lattice units per timestep (-u0.03), at which point the AdjustGrav () function would monitor and adjust the body force to maintain a constant fluid flow speed of 0.03 in the $x$-direction. The viscosity of the system would be set to 0.16 (lattice units) $)^{2} /$ timestep $(-n .16)$, and the diffusion coefficient of the one tracer component would be set to 0.03 (lattice units) 2 $^{2}$ timestep (-d.03) (if we had specified $-D .06$ as well, the program would have automatically allocated space for another component with diffusion coefficient of 0.06). The program would run for a maximum of 100000 timesteps (-s100000); at step 1999, a slug of solute would be injected at the upstream end (-g1999). At step 2000 $(-j 2000)$, the program would enter JustDisperse mode, freezing in the velocity distribution, and running the much faster (fixed flow field) tracer translation and collision functions. Since the $-t 10$ switch is also specified, at the time given by the $-j$ switch, the automaton would replicate the solids geometry 10 times in the $\mathrm{x}$-direction, allowing a much longer dispersion simulation (this was the method used to run the rectangular duct and SC sphere dispersion benchmarks, described in sections 3.3 and 3.4). To allow the maximum size calculation, JB_PH would first deallocate the memory used for the 19-vector carrier fluid, and reallocate this memory to new tracer arrays spanning the entire length of the automaton. The 
velocity field would not be replicated explicitly, but would use the MOD approach to pick local velocities, as described in section 2.

By default, JB_PH uses the 19-vector D3Q19 lattice for the carrier, and 6-vector lattices for tracers. However, by specifying -i19, the program will force use of 19 -vector lattices for the tracers.

\subsection{Character-Mode Display}

After JB_PH is invoked in a console (e.g., a DOS-box under Win95/NT, or an xterm under Unix/X-Windows), it provides a simple, character-mode graphics representation of the LB update process. The following discussion will focus on the Win95/NT version of the display. Currently, the display is set to refresh every 32 steps. The character-mode display is intended to provide a minimal capability, easily ported to diverse systems. The two sections below describe the development of a more sophisticated display capability.

The display has two basic modes: a normal $24 \times 80$ site "window" into the automaton, which can be panned over the automaton with the cursor keys; and a "Zoom" mode that zooms out to display the entire automaton, scaled to fit in the $24 \times 80$ view port. The modes are toggled with the " $Z$ " key. The display shows an $x-y$ plane at a fixed depth $z$ (layer); the displayed layer is changed with the PageUp and PageDown keys. The component displayed can be changed by pressing the "S" key; component 0 (the carrier fluid) displays the average fluid speed, whereas all other components display tracer concentrations. The "Esc" (escape) key or "Q" keys terminate the program.

\subsection{Passing Data to Asynchronous Graphics Programs: Plotfile.bin}

In addition to the simple character-based display, JB_PH "silently" generate a binary file that allows more flexible and colorful, "real-time" displays of automaton calculations. The plotfile.bin file is generated by the function MakePlot () (Appendix, listing 1) every $\mathbf{n}$ steps ( $\mathrm{n}$ is currently set to 32 , but it would be trivial to alter the source for variable intervals). In the next sub-section, an example will be given for a program that launches JB_PH, then operates asynchronously, displaying real-time images of the automaton progress.

The formats of plotfile.bin are specified in the "include" file plothead.h (Appendix, listing 2). The file is designed so its creation will impose a minimum performance penalty. As an test of the CPU burden, plotfile.bin was written every 32 steps when running the fastest LB tracer modes (JustDisperse ( ), 2.55 MUPs on a PII-400); the effect on performance was $<1 \%$. The first 4 bytes of the file contain the array PH_MACHINE [4], which specifies whether the machine that produced the file is big-endian or little-endian, and specifies the size (in bytes) of integers and floats on the creator. Thus, it is possible to read and interpret a plotfile.bin produced on a completely different architecture. The file then contains the LB step number, the size of the PLOTHEAD structure, and the PLOTHEAD structure itself. The latter structure specifies the type of data "slice" contained by the file: an $x-y$ slice; an $x-z$ slice; a $y-z$ slice; or a full $x-y-z$ "snapshot" of the time step. The structure contains some information on average speeds and fluxes in the system, along with scaling information useful for producing 
color images. After the structure, the tracer concentration or speed data are written in a manner consistent with the slice type.

\subsection{Example of Plotfile.bin Usage: a Win95/NT Interface}

Listing 3 (in the Appendix) gives a short Win95/NT program that launches JB_PH

asynchronously, awaits the creation of plotfile.bin, and displays the plotfile any time it changes. The program, pix_test.c $c$, passes any command-line switches on to JB_PH. To prevent pix_test from using too many system resources, the program uses timers, and checks for a new plotfile every 3 seconds. To ensure a speedy update of the display, pix_test writes its pixels to a Windows DIBSection, then flashes the DIBSection into the video buffer. The function GetNewPlot () (Appendix, listing 4) reads the plotfile into the PBUF buffer, and StretchPBUF2DIB () maps the data into the display buffer.

\subsection{JB_PH Data Structures}

To understand the algorithms used in JB_PH (Appendix, listing 5), it is necessary to review the storage of the particle distributions, flow speeds, and other quantities used in the calculations. The precision of the particle distribution arrays ( $F F$ or $f f$ ) and the velocity components ( $U$ or $\mathrm{uu}$ ) is controlled through the type specifications at the top of the program:

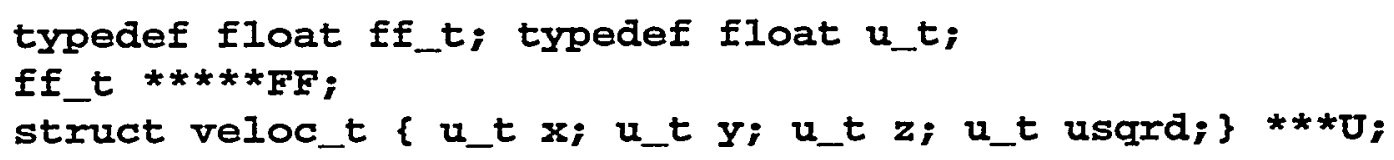

Thus by typing $f \_t$ and $u_{-} t$ as double, one can propagate increased precision throughout the program.

Like most arrays in JB_PH, FF and $\boldsymbol{U}$ are allocated dynamically. Thus there is no need to recompile the program to handle a larger problem size; however, dynamic allocation requires some special consideration for achieving efficiency, especially in light of C's well-known alias problems. As described in section 2, the FF array is allocated as FF [s] [i] [z] [y] [x], where $\mathbf{s}$ is the component index, $i$ is the vector number $(i=0,1, \ldots 18$ in D3Q19), and the remaining indices are the Cartesian coordinates of each spatial point in the LB lattice. The $U$ is allocated as an array of veloc_t $t$, so the $x, y$ and $z$ components at a spatial point $x_{1}, y_{1}, z_{1}$ are accessed as $\mathrm{U}[\mathbf{z} 1][\mathrm{y} 1][\mathrm{x} 1] \cdot \mathrm{x}, \mathrm{U}[\mathbf{z} 1][\mathrm{y} 1][\mathrm{x} 1] \cdot \mathrm{y}$ and $\mathrm{U}[\mathbf{z} 1][\mathrm{y} 1][\mathrm{x} 1] \cdot \mathbf{z}$.

Anyone attempting to modify JB_PH should be aware of a peculiarity in the handedness of the $x, y, z$ spatial grid, vs. the handedness of the velocity components. For historical reasons, the positive spatial $y$-axis points down in JB_PH, making a left-handed coordinate system; this is an artifact of the derivation of much of the code structure from earlier lattice gas codes, where the $y$ spatial axis was mapped directly into the rows of the video buffer. However, the velocity components are right-handed, with the positive $u_{\mathrm{y}}$ pointing "up", and all force and buoyancy components follow a right-handed system. 
Two other important structures are flags_struct and gravity. The former holds the flags that are parsed from the command line by SetFlags (). For each flag, the structure holds two components: an integer with a name like use_xxx, which specifies if the flag will be used; and the variable $\operatorname{sox}$, the value of the quantity. The use_mox is set to 1 by SetFlags () if the quantity is found on the command line, and its value falls within accepted limits; however, use_ $20 x$ may be reset to 0 by subsequent logic, since the main flags structure is global. The gravity structure begins with do_x, do_y and do_z; these are flags $(0$ or 1$)$ that determine if the body forces in the Cartesian directions will be adjusted to maintain the speed limits specified in uxximit, uyLimit and uzLimit. Currently, only uxsimit and uyLimit can be set by the command line (via the $-u$ and $-v$ switches). The XBody, yBody and $z B O d y$ specify the initial components of the body force; if the do_ $\mathbf{x}$, do_y or do_z are set, these are target values, i.e. once the speed limit in a particular Cartesian direction is reached, the body force may be reduced. The true body force at a given time step is given by txxBody, tyBody and tzBody; these values are set by the AdjustGrav () function. At each timestep, AdjustGrav () checks the average flow speeds on 1 to 3 control planes; if the average flow speeds on those planes differ from the speed limits, the corresponding $t$ ?Body are incremented up or down to approach the target speed. Each of the control planes has five coordinates to specify its position and extent; e.g., the control plane perpendicular to the $x$-axis (associated with the $x$-component of body force and the $u_{x}$ speed limit) has coordinates $x \mathrm{Ux}, \mathrm{y} 1 \mathrm{Ux}, \mathrm{y} 2 \mathrm{Ux}, \mathbf{z 1} \mathrm{Ux}$ and $\mathbf{z 2 U x}$.

In a complex geometry, it is very difficult to adjust the body forces to maintain a pre-specified flow speed. The problem is most difficult when there are large variations in the effective channel width (as in the SC flow problems described in sections 3.1 and 3.4), and when more than one velocity component is controlled. In such circumstances, there may be small oscillations in the flow speed, as AdjustGrav () tries to alternately increase and decrease the body forces, against an out-of-sync response in the flow speed. In such circumstances, it is useful to have means to "clamp" the body forces to fixed values, then "turn off" the AdjustGrav() algorithm. The gravity structure contains two parameters, stepstartclamp and stepstopclamp, that specify an interval to measure the average body forces and flow speeds before imposing the clamp. Optimal use of the clamping capability requires some foreknowledge of the flow problem; by default, JB_PH first checks the step specified by the $-j$ flag (if used), then checks the specified maximum step to determine clamping limits.

The xBuoy, yBuoy and zBuoy in gravity give the part of the information needed to calculate a position- and concentration-dependent buoyancy force. For example, the $x$-direction buoyancy force is $x$ Buoy $\cdot\left(\rho_{R E F}+\sum_{s=1} \alpha_{s} \cdot\left(\rho_{s}-\rho_{R E F}\right)\right)$ where the $\alpha_{s}$ is the molar expansivity of the sth component, and $\rho_{R E F}$ is a reference density. The $\alpha_{s}$ are stored in the alpha [MAXCOMPONTS] member of gravity.

\subsection{JB_PH Program Structure}

In this section, the basic structure of JB_PH is presented in a pseudocode outline. The actual source (Appendix, listing 5) contains many commented blocks that reflect experimental 
capabilities and functions. At the core of the program is the loop over update steps; each update is further divided into translation and collision functions, and forcing adjustments and imposition of boundary conditions.

defines

\section{JB_PH Pseudocode}

structure and type definitions;

function declarations;

Main \{

Open 1b_log.txt to log errors and unusual conditions;

Call setflags() to parse command line and set FlaGs structure values;

Adjust \# components, viscosities and diffusion coefficients for components, automaton size, disperse step, slug step, maximum steps, number of replications from flags;

Set default slug size, set Character display defaults;

Allocate and initialize FF (particle distributions), SOIID, D (array of velocity structures);

Initialize soIID by reading from file or by internal algorithm, and call setsolidType() to determine if solids are internal or bounding; Initialize the gravity structure $G ;$

Convert special flags (e.g. viscosity or diffusion) to $\tau$, and initialize TAO and RHO;

Perform initializations of fluids for special geometries;

select a dispersion mode, and point to the collision function;

Open the lb_data.txt file, to monitor program performance;

IOOP OVer STEP to MAXSTEP \{

Iook for keyboard input, and change display if required; iE (STEP==JUST_DISPERSE_STEP) \{

Recycle() memory if required, allocate new tracer units; \}

if (STEP<JUSM_DISPERSE_STEP) Translate() all components, including carrier;

else just Translate() tracers;

if (STEP==stepstartslug) \{

Either use setslug(), or read slug geometry from bitmap; save graphics of flow field (Savevect(), SaveUPpm()); \}

if (STEP<UUST_DISPERSE_STEP)

Use the normal Collide() function;

else Use the function JustDisp_ptr points to;

if (STEP<JUST_DISPERSE_STEP) \{

Use normal concentration boundaries (e.g. Xsetconc()); Apply AdjustGrav() to control fluid speed to limits; \}

else Use "JB" versions of concentration boundaries;

if ( (STEP \& PLOT_ANDER $)==0)\{/$ * PLOT_ANDER==31 currentIy */ Calculate and display ux average (UxAvg()); MakePlot() for plotfile.bin for asynch graph program; if(other special conditions) Write ppm files; \}

$3 \quad / *$ END LOOP */

Write a ux_uy.txt speed summary for debugging;

close log files, lb_data.txt file, any ppm list pointer files;

\} $/$ END Main */ 
Eunctions;

\subsection{Translation (Streaming) Algorithm}

As described in the section on efficiency, this version of JB_PH uses particle distribution arrays of the form $f f[s][i][z][y][x]$, with $s$ selecting the component, $i$ selecting the vector ( $i=0, \ldots, 18$ in D3Q19, or $i=0, \ldots, 5$ in the Cartesian 6-vector tracer lattice), and the $\mathbf{z}, \mathbf{y}, \mathbf{x}$ selecting the spatial position in the lattice. While this format may seem peculiar, it allows great simplification of the translation function, so the translation can be done "in-place" with minimal buffering. It also allows the tracers to mix 6-vector and 19-vector lattices with little effect on alignment and efficiency. After the program enters JustDisperse mode, only the tracers are translated.

The vector numbering scheme is given at the top of the JB_PH source, and is reprised below. The positive $u_{x}$ axis points to the right, and the positive $u_{y}$ axis points toward the top of the page, with the positive $u_{z}$ axis pointing out of the page ("above").

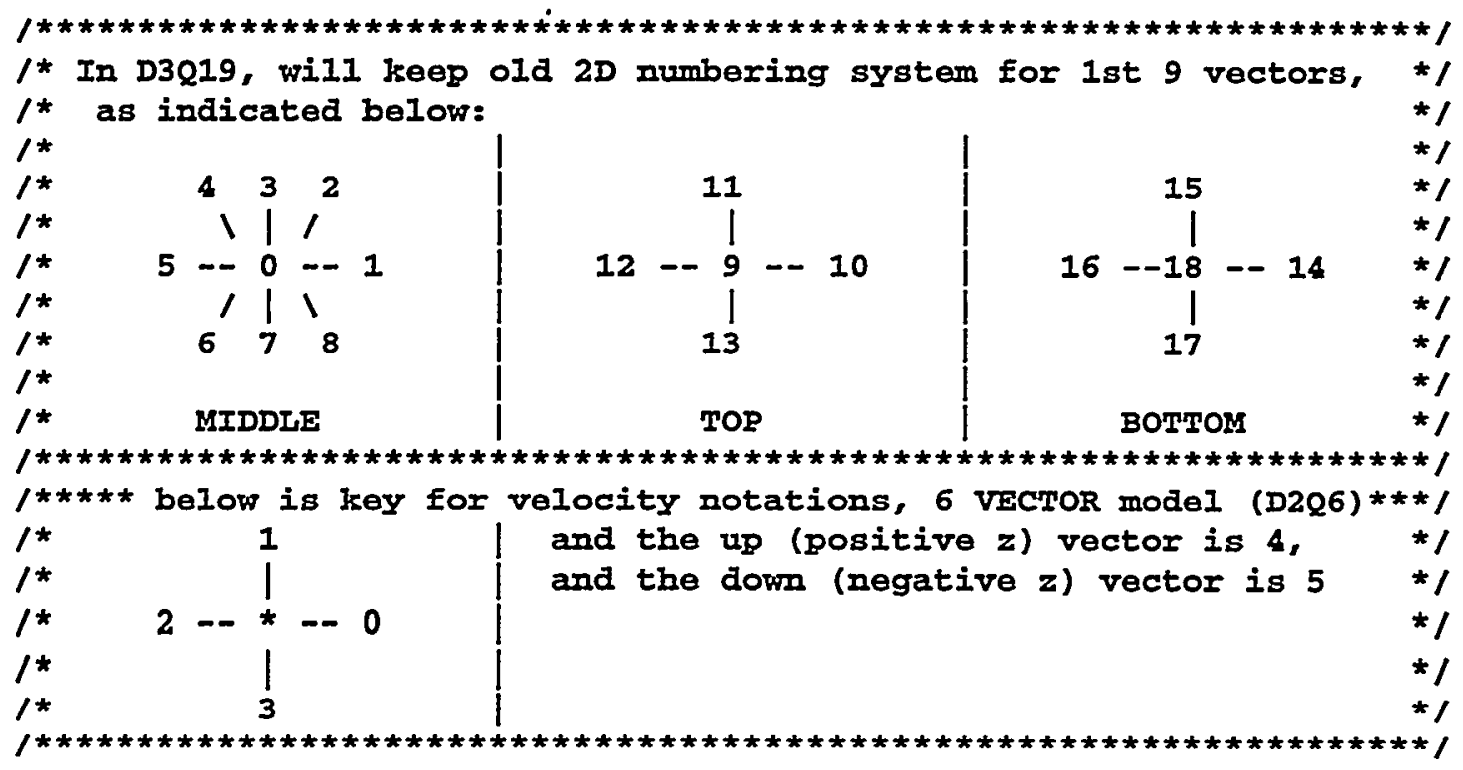

The function:

int Translate (ff_t $t * * * *$ const $f f$, int ncomponent, int nveloc[], int nlay, int nrow, int ncol, int sstart),

has an outer loop over s, the component index; withing this loop, the translation block for 19vector or 6-vector lattices is determined from the contents of nveloc [s]. For both lattice types, the boundaries wrap left-to-right, top-to-bottom, and side-to-side; e.g., a "particle" leaving the right side of the automaton at $\mathbf{x}=\mathrm{ncol}-1$ will be reinjected at ncol=0 with the same velocity.

The basic strategy of Translate () is to decompose each vector translation into a pointer swap and a physical movement in memory that references only one index. Some translations 
require only pointer swaps, and some require only physical movements in memory. Typically, at most only one pointer and one particle distribution need be buffered to effect a translation.

The character-mode function:

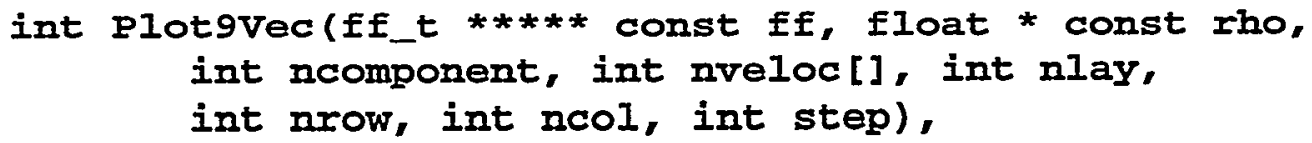

is useful for debugging the translation step. This simple routine prints the vectors in an $8 \times 8$ set of sites to the display, broken down into vectors in the $x-y$ plane, and vectors pointing below and above the plane. The Plot9vec () function is typically used in isolation, with the collision and concentration functions commented out, so one may track that the translations and boundary wraps are properly executed.

\subsection{Collision Algorithms}

The JB_PH source contains several collision functions (other specialized versions of the collison function are available from the author). The most general is Collide (), which can operate on an arbitrary number of components, with a non-steady $\mathbf{u}$ field, and with buoyancy. The code contains "hooks" for the imposition of complex wall collisions (in the form of the ziegler(), NoFlux () and NoFlux6 () calls ). However, Collide () has several features that reduce its efficiency: for example, the function applies buoyancy to an arbitrary number of components, requiring pre-calculation of densities, and the function requires a large number of indirections to fetch data from 5-dimensional arrays.

Much faster collision functions can be created for special conditions. Such conditions include: when there are only one or two tracers; when the tracers are 6-vector; or when the flow-field is steady state. For the last condition, JB_PH recognizes a special mode called JustDisperse. In this mode, only the tracer components are translated, and the function pointer JustDisp_ptr is used to select the dispersion function. In this version of JB_PH, two such functions are available: JustDisperse3D() and JustDisperse3D_A (). The former will disperse and arbitrary number of tracers, and per tracer, is up to 12 times faster than collide () (when collide () is running both the carrier and a single tracer). The JustDisperse3D_A () version is specially optimized for just one tracer. Because of memory limits, these two dispersion functions have nearly the same speed on the Intel $x 86$ chips; that is, the routines are both so efficient, that their speed is controlled by the RAM streaming limit of main memory.

\subsection{Controlling Fluid Speed and Gravity Adjustments}

As described above, the function AdjustGrav () allows one to keep the automaton at a specified average flow speed in a given direction, to simplify the attainment of pre-selected $P \boldsymbol{P}$ or $\boldsymbol{R} \boldsymbol{e}$. The function operates on a given Cartesian speed component (e.g. $u_{x}$ ) only if the flag for that component is set (e.g. do_ $\mathbf{x}>0$ ), and only before reaching the value of the stepstopclamp member of the gravity structure. The following discussion is restricted to the $x$-component; the treatment of the other directions is analogous. The average speed (ux) is measured on the 
specified control plane, and if this speed is greater or less than the uxiimit member, the txBody member is correspondingly adjusted. There is some logic to adjust the body force by small or large increments, to avoid generating undamped oscillations. After performing the adjustments, the function checks to see if the step is between the clamping limits, and if it is, the ux and txBody are added to a running average. At the end of the clamping period, if the average $u x$ (over the clamping period) is significantly different from the uxIimit, the average value of txBody is multiplied by uxIimit/(average ux over clamp period).

As with most discrete lattice methods, it is generally desirable to keep the maximum flow speed substantially below the numerical speed of sound in the automaton. We typically keep $U$ less than 0.01 to 0.05 for the D3Q19 carrier fluid.

\subsection{Concentration Boundaries}

Several functions are capable of imposing concentration boundaries on the system. The most common use of these boundaries is to inject a slug of solute, or to impose a particle sink, to prevent particles from leaving the outlet of a long channel, with subsequent reinjection at the inlet. The desired concentrations are passed to the functions by the array of double ConcList [MAX_COMP], where MAX_COMP is defined as the maximum number of components allowed in the system (currently 10). If a concList [i] $<0$, the ith entry is skipped by the called function.

The function:

int Setslug(ff_t $* * * * * f f$, char $* * *$ solid, int ncomponent, int nveloc[], int nlay, int nrow, int ncol, int $x 1$, int $\times 2$, int $y^{1}$, int $y^{2}$, int $z 1$, int $z 2$, double *conchist),

sets the ncomponent components of $f f[s][i][z][y][x]$ to the values specified in conchist, over the spatial domain within $\mathrm{x} 1$ to $\mathrm{x} 2, \mathrm{y} 1$ to $\mathrm{y} 2$, and $\mathrm{z} 1$ to $\mathrm{z2}$. Typically, SetSlug ( ) is called once, at the step specified by the $-g$ flag, to set up a Taylor-Aris dispersion problem. There are variants of this function, such as:

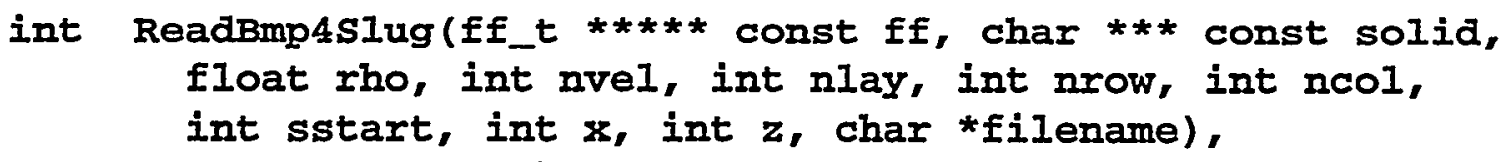

that read the slug geometry from a Windows bitmap (specified in filename), allowing one to inject slugs of nearly any geometry (figure 12 ). 


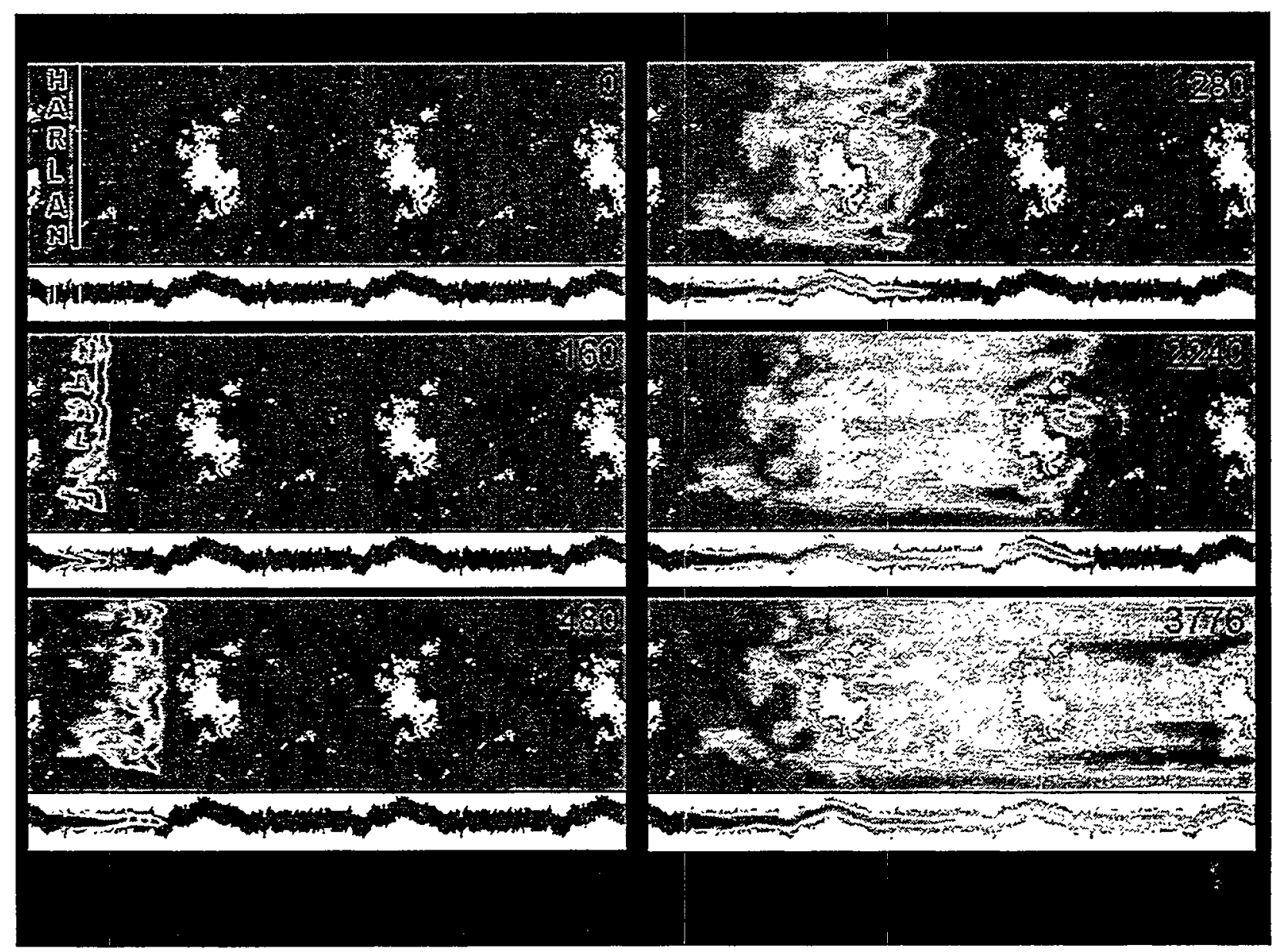

Figure 12. A slug of solute injected between walls of a rough fracture. Each frame shows a single timestep (indicated in black in the upper right corner), and two cuts through the fracture. The solids are in white; the initial carrier fluid is red; and the initial slug is blue fluid. At the top of the frame is a sub-horizontal cut, midway between the bounding surfaces, at $y=n$ row $/ 2$; at the bottom of each frame is an orthogonal cut, at $z=n$ lay/2. The slug was read from a bitmap via the ReadBmp4SIug () function, and the walls of the fracture were read with the Fractalsurface () function.

The function:

int Xsetconc(ff_t $* * * * *$ const ff, char $* * *$ const solid, int ncomponent, int nveloc[], int nlay, int nrow, int ncol, int $x$, int $y^{1}$, int $y^{2}$, int $z 1$, int $z 2$, double * const conclist),

sets the concentrations on the 2D rectangular section (perpendicular to the $x$-axis) specified by $\mathbf{x}$, $y 1$ to $y 2$, and $z 1$ to $z 2$; there are analogous functions for the $y$-and $z$-axis. The XsetConcJB () versions are used when the program is in JustDisperse mode (as determined by the $-j$ switch). 


\subsection{Measuring Velocities and Fluxes}

The function:

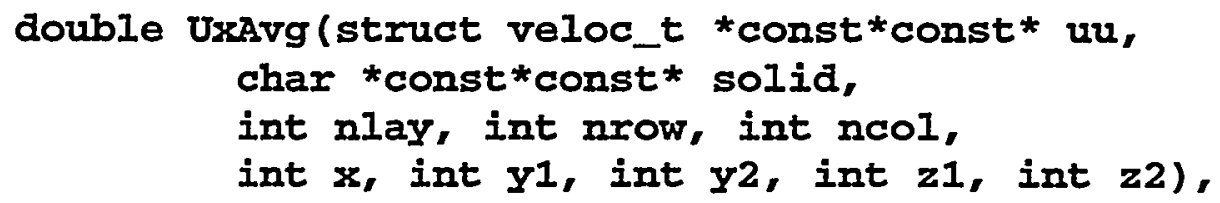

measures the $x$-direction component of the velocity field $\mathbf{u}$ over the rectangular region, perpendicular to and intersecting the $x$-axis at $x$, and bounded by $y 1$ to $y 2$, and $z 1$ to $z 2$. The function returns the average value of the fluid speed for open spaces only. That is, the function does not return the superficial (Darcy) velocity, though it could be modified easily to do so. The functions UyAvg ( ) and UzAvg ( ) perform similar services for the $y$-and $z$-components of velocity.

Similarly, the function:

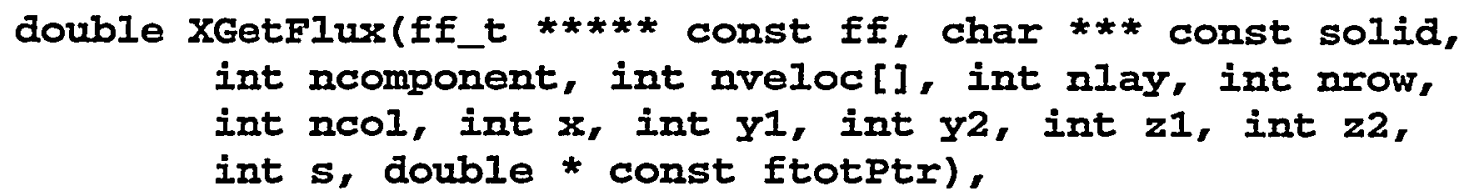

returns the fluence of the component s through the specified rectangular region, inclusive of the endpoints. Specifying $s==0$ effectively returns a net velocity. The total amount of the component in the rectangle is returned in ftotPtr. To obtain true flux, the returned fluence must be divided by $(|y 2-y 1|+1) \cdot(|z 2-z 1|+1)$. There are $y$ - and $z$-analogues for this function as well.

\subsection{Generating PPM, Vector, and Movie Files}

Several functions in the JB_PH source can generate graphics files for subsequent display or incorporation into MPEG movies. Any function whose name contains "Ppm" generates portable pixel maps, as defined in the Unix utilities "pbmplus". These files have an extremely simple format, and are easily converted to Windows bitmap files by programs such as Lview Pro. The beginning of each ppm is written by a statement similar to:

fprintf (fp, "P6 $1012 \% d \% d \backslash 012255 \backslash 012 \pi$, columns, rows);

where $\mathrm{fp}$ points to the open binary ppm graphics file. This statement writes the magic string "P6", followed by a Unix newline, then the number of columns and rows in the map, separated by a space and followed by a newline, then the maximum color per byte ( 255 in this case), followed by a newline. Immediately following, the RGB data are written ( 3 bytes to an RGB pixel), row by row, for a total of 3 *columns * rows bytes of pixel map data.

Each ppm file has a name Cddhhnn.ppm, where where $\mathbf{C}$ is a character denoting the ppm file type (currently $B, U$ or $\mathbf{T}$, corresponding to $B=b i t m a p, ~ U=$ flow speed, or $T=$ three-dimensional), 
dd is the day of the month, $\mathrm{hh}$ is the hour the list file was created, and $\mathrm{nnn}$ is $\mathrm{s}$ number from 000 to 999 . With the exception of SaveuPpm (), each ppm function returns a pointer with a name like (FIIE *) b_list_ptr; this pointer denotes a file that contains a list of all ppm files generated during the run. The list file helps one sew the ppm files together into an MPEG movie, via utilities like the freeware program cmpeg. The name of the list file will be Cddhh. Ist. If more than $1000 \mathrm{ppm}$ files are created in a run, a new list file is created with the new current day and hour.

Currently, the following ppm-producing functions are available in the JB_PH source:

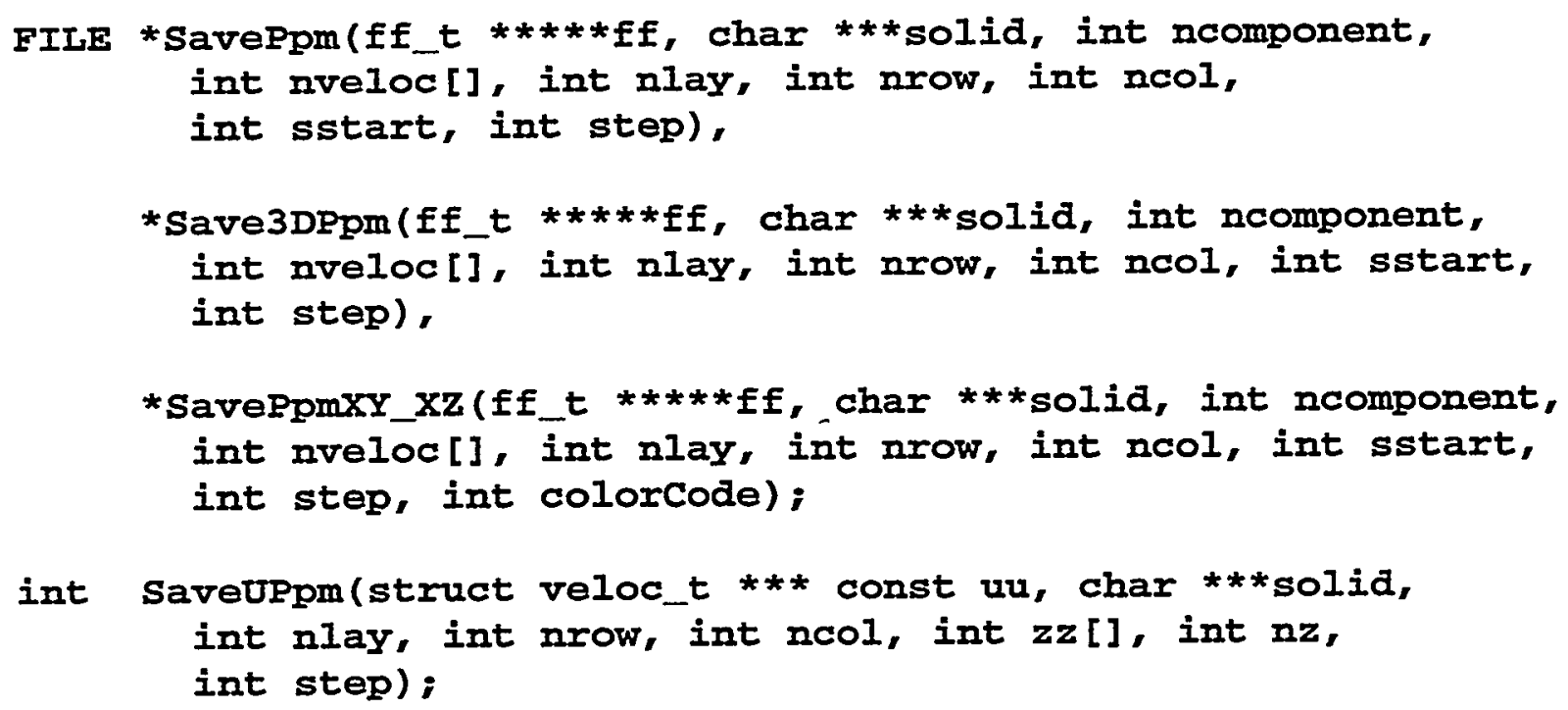

The differences among the first three are in the manner of portraying "cuts" through the $x-y-z$ space, and in the options for color schemes; all plot the concentration of the tracer with index sstart. The first function takes cuts at planes $z \sim n l a y / 2$ and $x \sim n c o l / 2$, and arranges them side-by-side on the page; the second plots the concentrations on the $x=0, y=0$ and $z=0$ faces, for a semi-3D appearance arranged in an isometric perspective (figure 13). The third makes cuts at planes $z \sim n l a y / 2$ and $y \sim n r o w / 2$, arranging the latter above the former on the page. The SavePpmXY_Xz ( ) function is most useful for portraying dispersion and reaction in long, flat channels, with dimensions like figure 12 . 


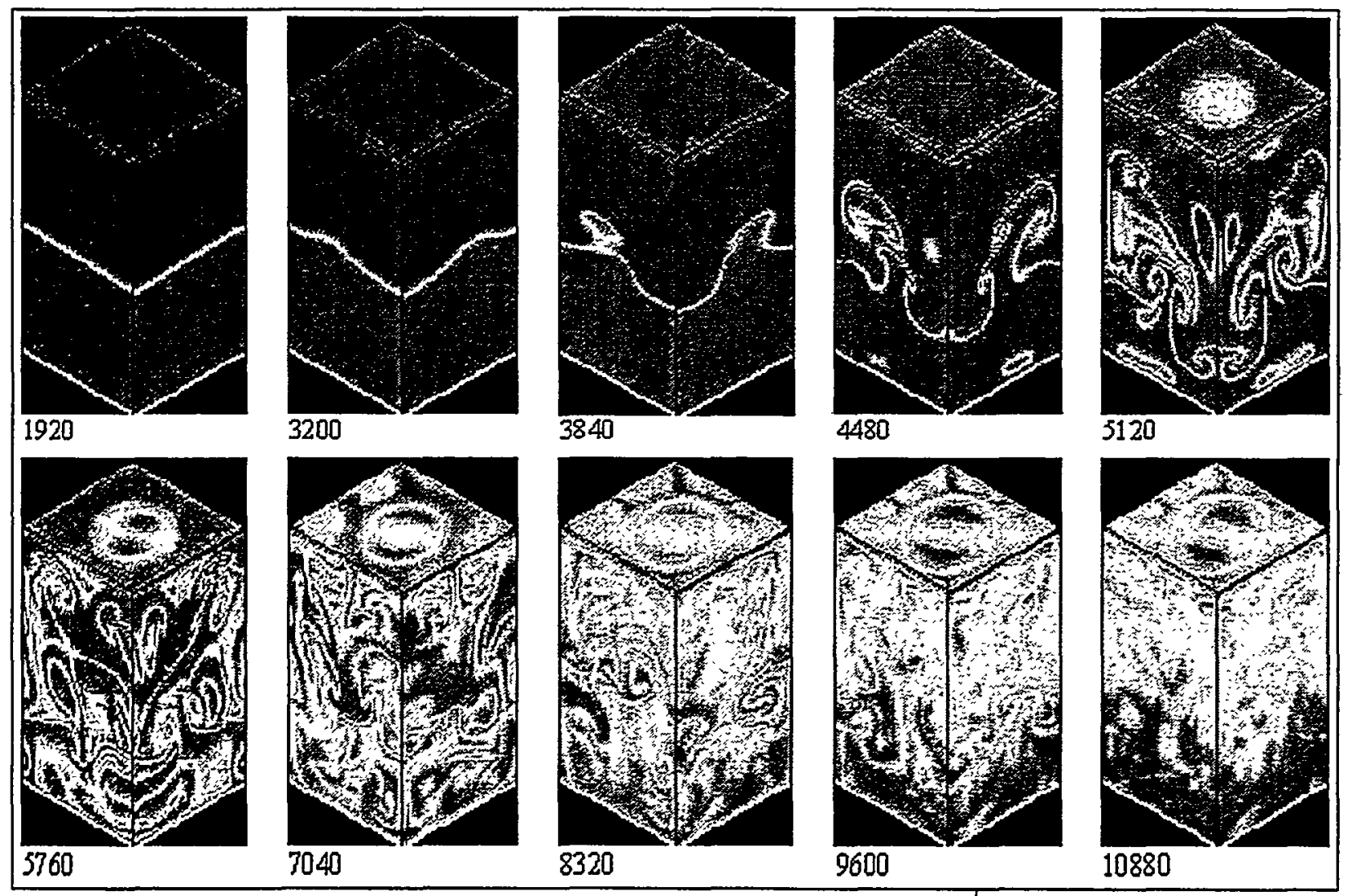

Figure 13. Images of the Rayleigh-Taylor instability, plotted with the function Save3DPpm (). A denser blue fluid initially overlies a red fluid; the two overturn in mushroom-shaped patterns. The timestep is shown at the bottom of each frame.

The function SaveUPpm () does not return a file pointer, and is designed to give a velocity snapshot at each of the NZ planes specified as z-indices in the Zz [] array. The snapshot is typically performed once, just before freezing in a flow pattern for the JustDisperse modes. The NZ images are arranged vertically on the page, with higher $z$ values successively farther down-page.

The function:

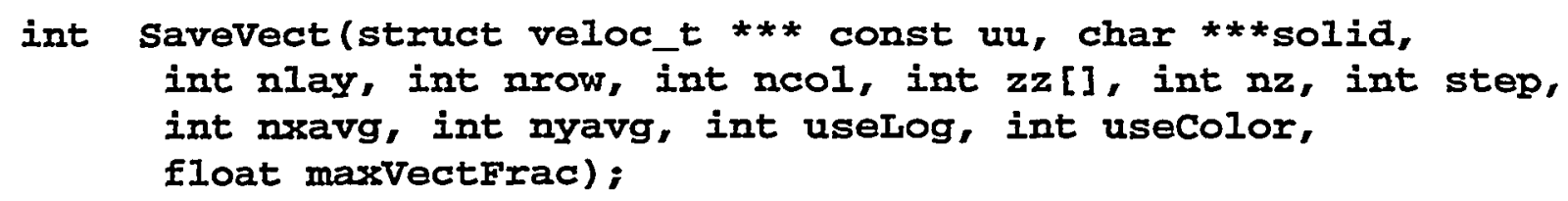

is analogous to SaveUPpm (), except it produces vector plots (like those in figure 9) instead of ppm bitmaps. The vector plots are written in the Hewlett-Packard graphics language (HPGL), and the file names have the form vadhhnnn.zzz, where $v$ denotes vector (and does not change), dd is the day of the month, $h \mathbf{h}$ is the hour, $n$ nn is the number of the file in the sequence, and $\mathbf{z z z}$ is the $z$ position of the cut in the lattice. Unlike SaveUPpm(), 
Savevect () creates a separate file for each cut, since the vector plots are each sufficiently complicated as to swamp many conversion or import utilities in word processors. Current versions or Microsoft Office (after 1997) do not have built-in HPGL import utilities. However, the files can be converted to PostScript with the free hp $2 \times x$ package, or with commercial conversion utilities. In principal, it should be simple to change the source code to write Windows metafiles instead of HPGL. The nxavg and nyavg tell the routine how many pixels to average (in $x$ - and $y$-directions), and the uselog chooses linear (uselog $=0$ ) or pseudologarithmic scaling (useLog $>0$ ) for vector lengths (the latter is actually a fourth-root scaling, and is useful to emphasize flow in small recirculation zones at modest $\boldsymbol{R e}$ ). The usecolor parameter determines if the function will color the vectors to correspond to their lengths (with shortest vectors black, and longest red), and maxvectFrac () gives the fraction the fraction of the largest LB array dimension (e.g. ncol, number of columns) that will be allowed for the longest vector in the plot.

A current limit of the Ppm functions is that they typically allot a maximum RGB_MAX_SIZE of 8192 pixels to represent a row. For creating larger plots, this size may simply be increased, or logic added to not plot beyond the end of the array. Alternatively, the arrays could be allocated dynamically and deallocated with each call.

\subsection{Measuring Dispersion: The Mean_std.txt File}

To measure dispersion by the method of moments ${ }^{15}$ [EQ], it is necessary to calculate the moments of the solute distribution as functions of time. The function Meansta () calculates the total solute in the automaton, and the $1^{\text {st }}$ moment and standard deviation of the solute distributions in the $x$ - $y$ - and $z$-directions at the specified time increments, and writes them to an ASCII file called mean_sta.txt. The latter file is opened and closed with every call, so one can make and edit complete copies of the file while JB_PH is running in another window. The file is always opened in "append" mode, and should be pruned periodically. With each JB_PH run, a header is written to Mean_std.txt, containing the date, executable name and path, the component used for the moment calculations, and the size of the automaton. Then a tabbed header is written with the column titles, and each subsequent line of the file contains the parameters: step, fSum, xMean, xStd, yMean, yStd, zMean, and zStd, which correspond to the step number, total sum of solute for the component selected in the call, mean position ( $1^{\text {st }}$ moment) in the $x$-direction, standard deviation in the $x$-direction, etc. It must be emphasized that xsta, $\mathbf{y s t a}$ and zstd are standard deviations, not variances, and must be squared to obtain the second moments necessary for equation (10). The fsum is useful for determining if a slug of solute has begun to "fall off the ends" of the automaton at the end of the run, or if there is something amiss with the boundary conditions. Typically this quantity stays constant to the $5^{\text {th }}$ decimal place for the bulk of a Taylor-Aris dispersion run.

\subsection{Reading Solids Geometries from Miscellaneous Files}

It is quite simple to read a solid geometry into JB_PH, particularly if the input file is a bitmap or is otherwise organized in $x-y-z$ voxels. In general, the process involves initializing the SOLID [z] [y] [x] array to 0 , then reading in the geometry file, and placing a -1 in the SOIsD array wherever a solid occurs in the geometry file. Then the SOIID array is passed to the 
function SetSolidType (), which determines if solids are at solid-fluid interfaces, or are completely internal.

In some cases, the solid geometry is specified as bounding surfaces, or surfaces that need to be scaled before the mapping into the SOLID array. An example is given in the function:

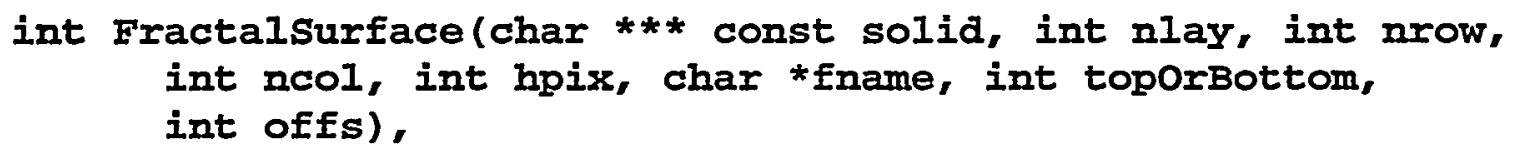

which takes fractal surface files created by Stephen Brown's fractal . for program, ${ }^{29,30}$ and draws the surface into the SOIID array. The parameter hpix is the number of pixels (rows) that correspond to the maximum relief in the fractal file; hpix should generally be less than (nrow - offs). The solids are drawn from offs into the site volume, parallel to the $y$ direction. If toporBottom is 0 , the surface is taken as the "bottom" of the fracture, and solids are filled from the fracture surface down to offs; if toporBottom $>0$, the surface is taken as the top face of a fracture, and solids are filled from offs down to the surface. Thus by pairing the calls to Fractalsurface (), one can create complicated and asymmetric fractures. The last call should be followed by a call to setSolidType ().

\subsection{Generating Pseudo-Random Spherical Packings}

The function:

\section{int setFCCSolids(char ***solid, int nlay, int nrow, int ncol, int zrep, int yrep, int xrep, float rslop, float cslop, float rbias, int seed),}

fills the solid array with a set of slightly randomized, quasi-FCC (face centered-cubic) spheres. The zrep, yrep and xrep are the number of FCC cell reps per nlay, nrow and ncol, respectively; there are 4 complete points per FCC cell. rslop is the fractional, random slop applied to the grain radius; cslop is the slop applied to the $x, y, z$ position of a grain center. Both rslop and cslop should be between 0.0 and 0.5. The rbias is the bias applied toward smaller grain diameters, vs. larger diameters; it is a float from 0.5 to 1.0 , such that 0.5 provides even distribution of radii above and below mean, and 1.0 biases all radii to be below the ideal radius. The seed argument is an integer seed used to initiate the random number generator. If Mrand () is used, seed is a positive integer from 1 to $2^{31}-1$ (seed CANNOT be 0 ). Note this function call must be followed by a call to SetSoliatype (). SetFCCSolids () was used to generate the solids pattern in figure 14. 


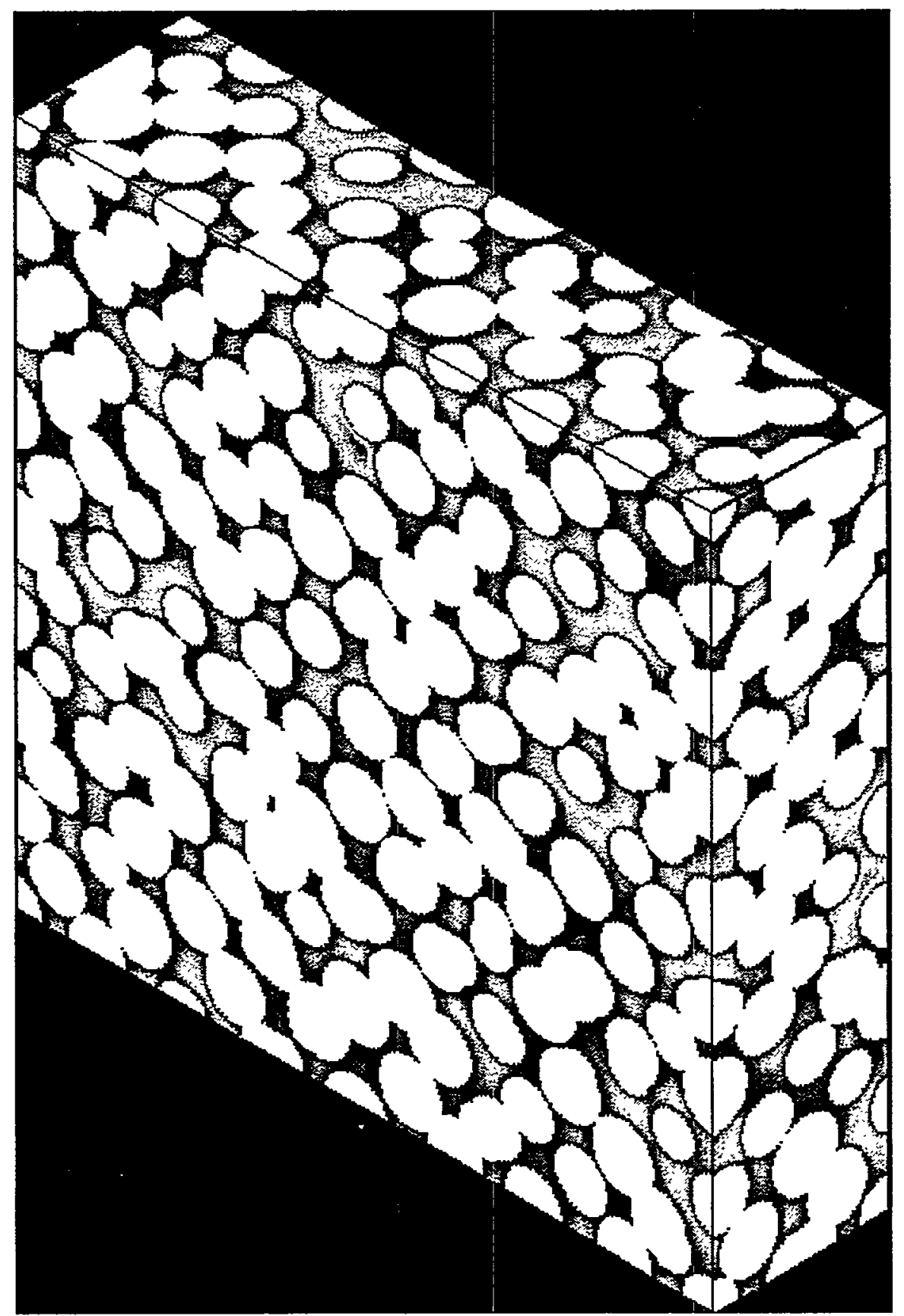

Figure 14. A pseudo-randomized solids pattern created by the SetFCCSolias () function. The solids are in white, and the colors show an equilibrium flow field, with the lowest flow speeds corresponding to the "coolest" colors (e.g. black), and the warmest colors (red) corresponding to highest flow speeds.

\section{History of LB Code Development}

The JB_PH code draws on numerous sources. Under the Department of Energy's Basic Energy Sciences (BES) program, the author developed lattice gas codes from 1991-1995. Several algorithms in JB_PH, such as the Ppm functions and AdjustGrav ( ), are modifications of older LGA routines. Under a BES program to study double-diffusive convection (1996-1998), 
the author wrote several 2D LB codes, then the program 3D.c, which included most of the functionality of JB_PH, including the 3D Collide () and Translate () functions.

However, the design of 3D.c benefited from the authors' experience working on 2D codes under a Sandia National Laboratories LDRD program (principally from March 1997 to March 1998). In particular, the LDRD program allowed the author to test accuracy and boundary conditions with rigor, and to optimize and fine-tune the routines. Interactions with David Noble, under the LDRD program, allowed development of the reduced-vector methods for efficient modeling of tracer dispersion and reaction. Finally, the concept of recycling memory and cloning the tracer geometries was developed under BES, in support of the double-diffusive convection program, but was fine-tuned and tested in part under the LDRD. 


\section{Conclusions}

With simple optimizations, the BGK LB method is fast enough, on a single-CPU computer, for modeling complex solids geometries containing millions of nodes. However, care is required to prevent numerical anomalies (such as denormal generation) from reducing overall performance. Good agreement was obtained with four benchmark problems. Simple bounce-back wall conditions are less accurate than other methods, ${ }^{9}$ but yield acceptable errors $(\leq 4 \%)$, given the inherent uncertainty of dispersion experiments in complex geometries.

\section{References}

${ }^{1}$ H.W. Stockman, A Lattice-Gas Study of Retardation and Dispersion in Fractures: Assessment of Errors from Desorption Kinetics and Buoyancy, in Water Resources Res., vol. 33 (\#8), pp.1823-1832, 1997.

${ }^{2}$ H.W. Stockman, C. Li and J.L. Wilson, A Lattice-Gas and Lattice Boltzmann Study of Mixing at Continuous Fracture Junctions: Importance of Boundary Conditions, in Geophys. Res. Lett. vol. 24, pp. 1515-1518, 1997.

${ }^{3}$ C. Wels, L. Smith and T.T. Vandergraaf, Influence of Specific Surface Area on Transport of Sorbing Solutes in Fractures: An Experimental Analysis, in Water Resources Res., vol. 32, pp. 1943-1954, 1996.

${ }^{4}$ A. Tsuda, W.J. Federspiel, P.A. Grant, Jr. and J.J. Fredberg, Axial dispersion of inert species in alveolated channels, in Chem. Engng. Sci., vol. 46, pp. 1419-1426, 1991.

5 H. Wong and T.-Y. T. Lee, Thermal Evaluation of a PoerPC 620 Microprocessor in a Multiprocessor Computer, in IEEE Trans. on Components, Packaging, and Manufacturing Tech. Part A, vol. 19, pp 469-477, 1996.

6 Y.H. Qian, D. d'Humières P. Lallemand, Lattice BGK Models for Navier-Stokes Equation, in Europhys. Lett., vol. 7(6 BIS), pp 479-484, 1992.

${ }^{7}$ N. Martys and H. Chen, Simulation of Multicomponent Fluids in Complex Three-Dimensional Geometries by the Lattice Boltzmann Method, in Phys. Rev., vol. E 53, pp. 743-750, 1996.

${ }^{8}$ E.G. Flekk $\varnothing y$, U. Oxaal, T. Feder and T. Jøssang, Hydrodynamic Dispersion at Stagnation Points: Simulations and Experiments, in Phys. Rev., vol. E 52, pp. 4952-4962, 1995.

9 D.R. Noble, Lattice Boltzmann Study of the Interstitial Hydrodynamics and Dispersion in Steady Inertial Flows in Large Randomly Packed Beds. PhD thesis, University of Illinois Urbana-Champaign, 1996. 
${ }^{10}$ D. Wolf-Gladrow, A lattice Boltzmann Equation for Diffusion, in J. Stat. Phys. vol. 79, pp. 1023-1032, 1994.

${ }^{11}$ D. Muders, Three-Dimensional Parallel Lattice Boltzmann Hydrodynamic Simulations of Turbulent Flows in Interstellar Dark Clouds, Ph.D. thesis, Universität Bonn, 1995.

${ }^{12}$ S.P. Morse, E.J. Isaacson and D.J. Albert, The 80386/387 Architecture. John Wiley \& Sons, New York, 1987.

13 A.A. Zick and G.M. Homsy, Stokes Flow through Periodic Arrays of Spheres, in J. Fluid Mech. vol. 115, pp. 13-26, 1982.

${ }^{14}$ R.B. Saeger, L.E. Scriven and H.T. Davis, Transport Processes in Periodic Porous Media, in J. Fluid Mech. vol. 299, pp 1-15., 1995.

${ }^{15}$ R. Aris, On the Dispersion of a Solute in a Fluid Flowing through a Tube, in Proc. Royal Soc. vol. 235A, pp. 67-77, 1956.

${ }^{16}$ S.J. Chen, D. Martínez and R. Mei, On Boundary Conditions in Lattice Boltzmann Methods, in Phys. Fluids vol. 8, pp. 2527-2535, 1996.

17 L. Ginzbourg and D. d'Humières, Local Second-Order Boundary Methods for Lattice Boltzmann Models, in Jour. Stat. Phys., vol. 84, pp. 927-971, 1996.

18 A.C. Lasaga, in Kinetics of geochemical processes, Rev. in Mineralogy 8, ed. A.C. Lasaga et al. (Mineralogical Soc. America, Washington DC, 1981) p. 135.

${ }^{19}$ X. He and L.-S. Luo, Theory of the Lattice Boltzmann Method: From the Boltzmann Equation to the lattice Boltzmann Equation, in Phys. Rev. E, vol. 56, pp. 6811-6817.

${ }^{20}$ P.C. Chatwin and P.J. Sullivan, The Effect of Aspect Ratio on Longitudinal Diffusivity in Rectangular Channels, in J. Fluid Mech, vol. 120, pp. 347-358, 1982.

${ }^{21}$ T.T. Vandegraaf, D.J. Drew, D. Archambault and K.V. Ticknor, Transport of Radionuclides in Natural Fractures: Some Aspects of Laboratory Migration Experiments, in Jour.

Contaminant Hydrology, vol. 26, pp. 83-95, 1997.

${ }^{22}$ Y. Fujikawa, F. Masami, D.J. Drew and T.T. Vandegraaf, Analysis of the Migration of Instantaneously Injected Cesium in Artificial Fractures of Lac du Bonnet Granite, Manitoba, in Jour. Contaminant Hydrology, vol. 14, pp. 207-233, 1993.

D.J. Gunn and C. Pryce, Dispersion in Packed Beds, in Trans. Inst. Chem. Engrs., vol. 47, pp. T341-T350, 1969.

${ }^{24}$ A. Eidsath, R.G. Carbonell, S. Whitaker and L.R. Herrmann, Dispersion in Pulsed Systems -- 
III Comparison between Theory and Experiments for Packed Beds, in Chem. Engng. Sci., vol. 38, pp. 1803-1816, 1983.

25 D.L. Koch, R.G. Cox, H. Brenner and J.F. Brady, The Effect of Order on Dispersion in Porous Media, in J. Fluid Mech., vol. 200, pp. 173-188, 1989.

26 J. Salles, J.-F. Thovert, R. Delaney, L. Prevors, J.-L. Auriault and P.M. Adler, Taylor Dispersion in Porous Media. Determination of the Dispersion Tensor, in Phys. Fluids, vol. 5(10), pp. 2348-2376, 1993.

27 C.K. Lee, C.-C. Sun and C.C. Mei, Computation of Permeability and Dispersivities of Solute and Heat in Periodic Porous Media, in Int. J. Heat Mass Transfer, vol. 39, pp. 661-676, 1996.

${ }^{28}$ M. Kaviany, Principles of Heat Transfer in Porous Media. Springer-Verlag, New York, pp 178-179, 1991.

29 S.R. Brown, Simple Mathematical Model of a Rough Fracture, in J. Geophys. Res., vol. 100, pp. 5941-5952, 1995.

${ }^{30}$ S.R. Brown and R.L. Brubn, Formation of Voids and Veins During Faulting, in J. Struct. Geol, vol. 18, pp. 657-671, 1996. 


\section{Appendix: Code Listings}

\section{Listing 1. Makeplot().}

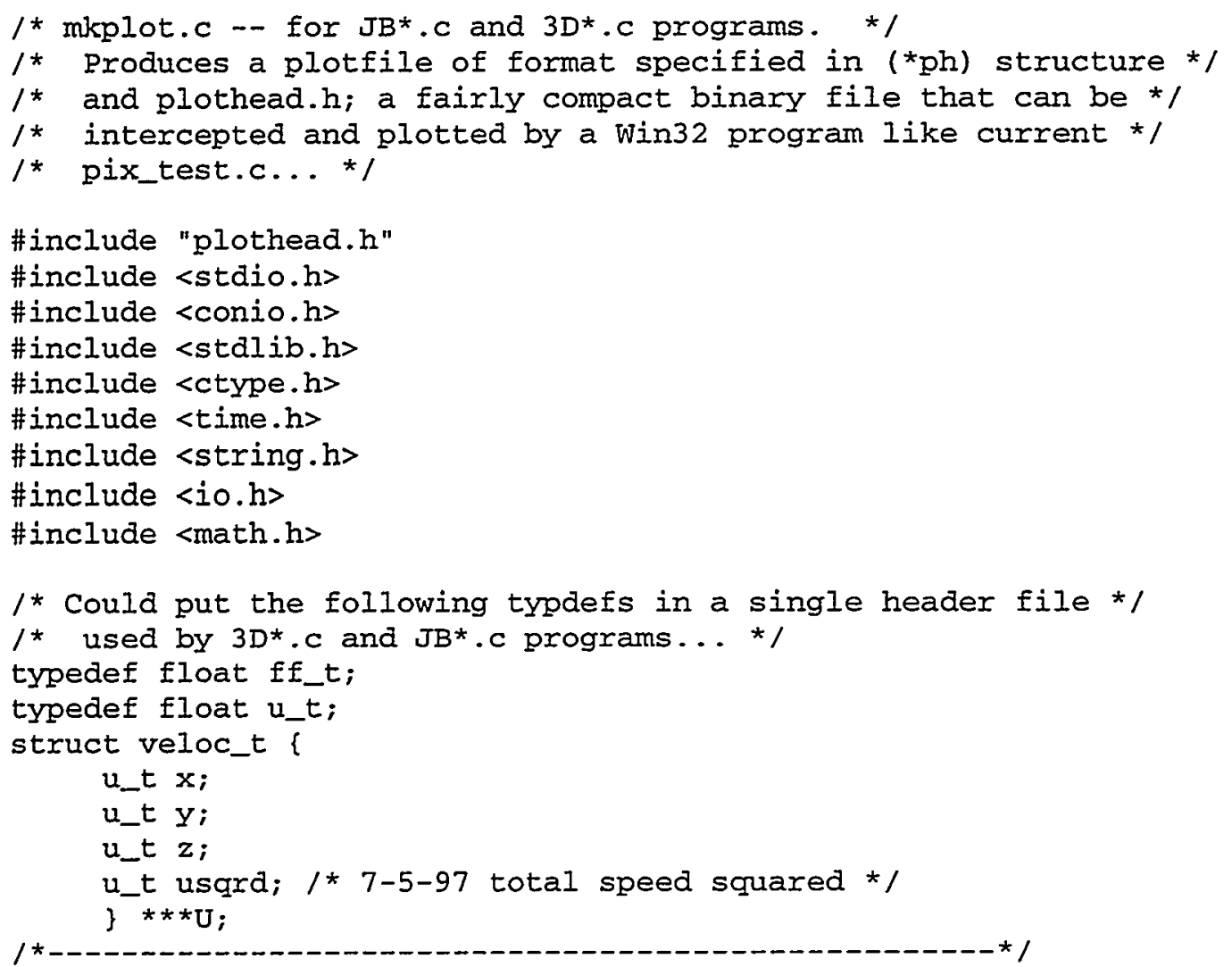


int nlay, int nrow, int ncol, int ncol_old, int step, struct PLOTHEAD *ph, int sliceType, int sizeofDatum,

\{ int iComponent, int index)

static float *pfBuf=NULI; 1 * holds a complete slice, densities or speeds */ static unsigned char *puBuf=NULI; $/$ * holds one "line" of data to write, used */ $1 *$ only if this is an unsigned char type file (ph->sizeofDatum=1) */

static int nfBuf=0, nuBuf=0; $/ *$ size of buffers above IN BYTES * static char *fname = "plotfile.bin";

int $i, k, x, y, z, s, x b, y b, z b, b C o l, b R o w, b l a y ; / *$ latter are into buffer */

int nfBufold, nuBufold;

int nvel;

ff_t $* * * * f f s$;

int ioffs, iioffs; $/$ * used to speed indexing into pfBuf */

float rho, rhomin, rhomax, fMul, fDiv;

/* rho must be same type as PH_FSOLID !! */

float fVal; /* 12-21-97 */

FILE * fp;

int istatus;

int $x x$, iindex; $l^{*} x x$, index for ncol_old stuff $* /$

int sizeofPlotHead;

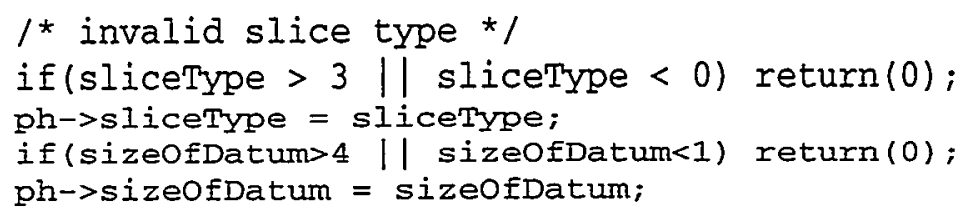


break;

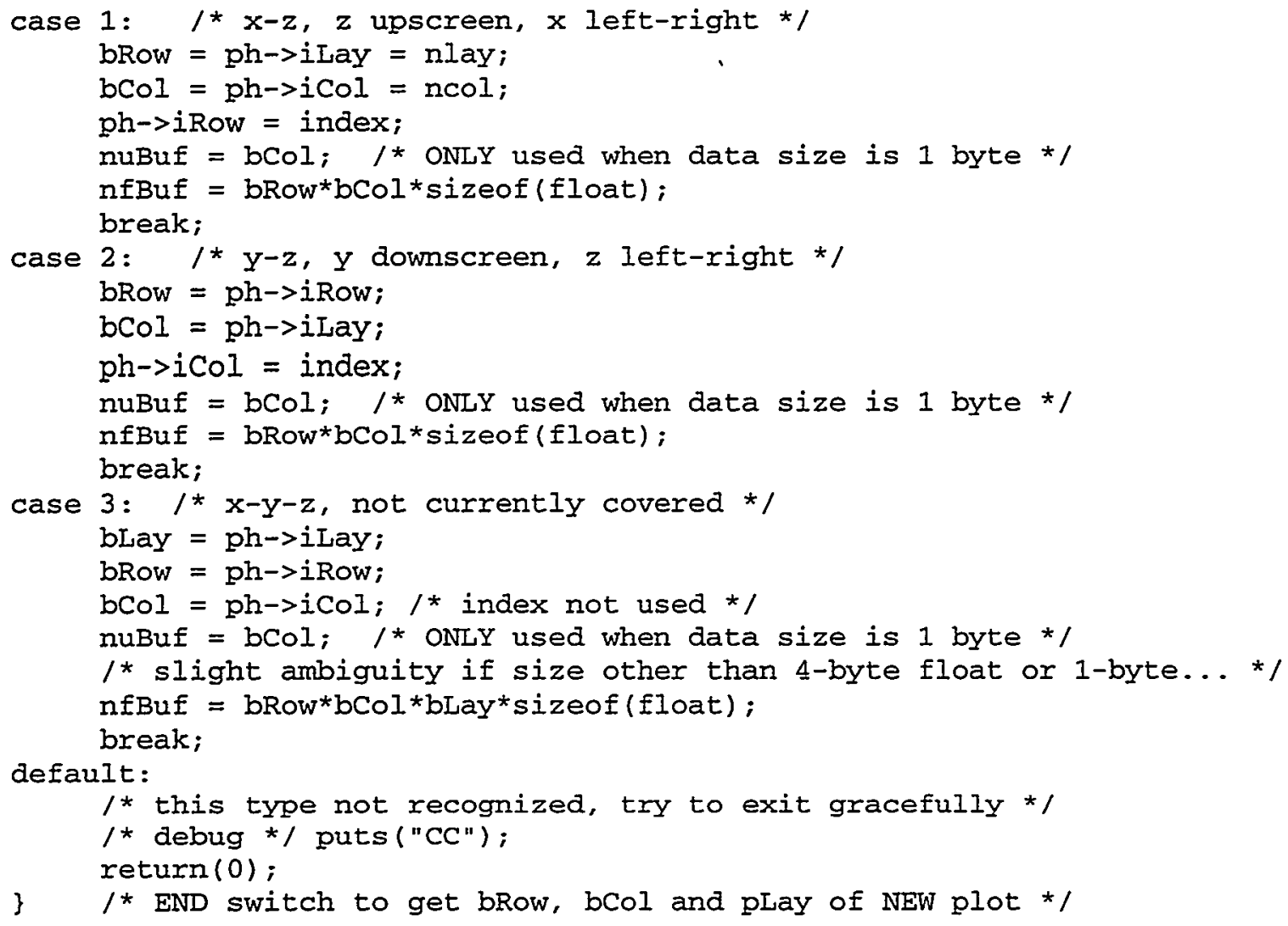

I* Next see if we need to reallocate. The internal buffers are $1 D * /$

$1 *$ arrays. The unsigned char array is used only if the sizeodDatum */

$1 *$ is 1 ; in that case, the normalized density buffer nfBuf is */

1 * renormalized to 1 byte values bRow by bRow, and written to disk... */

if (puBuf==NULL || pfBuf==NULL || nuBuf>nuBufold || nfBuf>nfBufold) \{

if(puBuf) Eree(puBuf); if (pfBuf) free(pfBuf);

puBuf $=\operatorname{malloc}($ nuBuf); pfBuf $=\operatorname{malloc}($ nfBuf)$;$

if(!nuBuf) $\{$ if(nfBuf) free(pfBuf); return(0);\}

if(!nfBuf) \{ if(nuBuf) free(puBuf); return(0);\}

if $(s)$ s 3

/* Process case of component $>0$; i.e. a concentration, not */

$1 *$ a speed. For slicetype $=0,1,2$, index specifies the fixed index. */ rhomin $=1.0 \mathrm{e} 30$; rhomax $=-1.0 \mathrm{e} 30$;

switch (sliceType) \{

case $0:$ /* xy slice $000000000000000000000000000000000000000000000 * /$

for $\left(y=0 ; y<\right.$ nrow; $\left.\mathrm{y}^{++}\right)$\{

ioffs $=y^{*}$ bCol

for $(x=0 ; x<n c o l ; x++)\{$

if (solid [index] $[y][x])$ rho $=$ PH_FSOLID;

else \{

for (rho=0.0f, $i=0 ; i<$ nvel; $i++)\{$

rhot= ffs $[i][$ index $][y][x]$;

\}

if (rho<0.0f) rho $=0.0 f$;

if ( $r$ hos=rhoMax) rhoMax = rho;

if (rho<=rhomin) rhomin = rho;

\} 


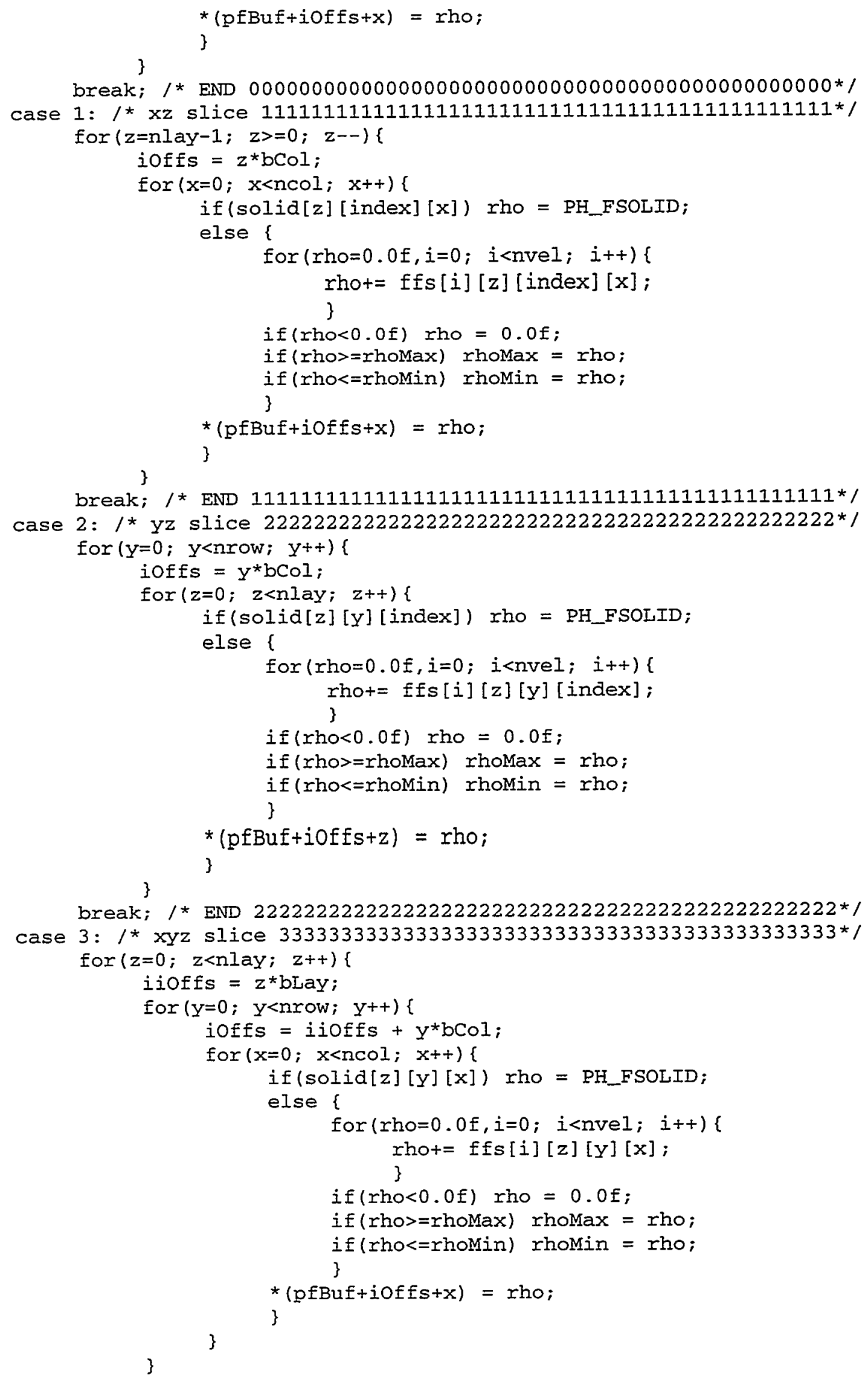



else \{

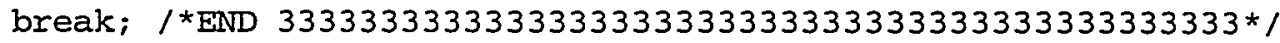

1* don't need default, filtered out above */

\}$/ *$ END switch() on sliceType */

\} $/ *$ END ifl) on $s>0$ (concentration, not speed) */

I* Process case of component $==0$; i.e. a speed squared. */

$1 *$ For slicetype $=0,1,2$, index specifies the fixed index. */

1* Remember that only the ncol_old part of uu array exists, so *

$1 *$ we must implicitly mod $x$ by ncol_old... */

1 * NOTE can't be negative... */

rhomin $=1.0 \mathrm{e} 30 ;$ rhomax $=-1.0 \mathrm{e} 30$;

switch (slice'Type) (

case $0: 1^{*} \mathrm{xy}$ slice $000000000000000000000000000000000000000000000 * /$

for $(y=0 ; y<$ nrow; $y++)\{$

ioffs $=y^{*}$ bCol

for $(\mathrm{xx}=\mathrm{x}=0 ; \mathrm{x}<\mathrm{ncol} ; \mathrm{x}++, \mathrm{xx}++)\{$

if $\left(x x==n c o l \_o l d\right) \quad x x=0$;

if (solid[index] $[y][x]$ ) rho = PH_FSOLID;

else \{

rho $=$ uu $[$ index $][y][x x]$.usqrd;

if (rho>=rhomax) rhomax = rho;

if (rho<=rhomin) rhomin = rho; \}

$*($ pfBuf + iOffs $+x)=$ rho;

\}

\}

break; /* END 0000000000000000000000000000000000000000000000 /

case 1: /* xz slice 111111111111111111111111111111111111111111111*/

for $(z=$ nlay $-1 ; z>=0 ; z--)\{$

iOffs $=z^{*}$ bCoI;

for $(\mathrm{xx}=\mathrm{x}=0 ; \mathrm{x}<\mathrm{ncol} ; \mathrm{x}++, \mathrm{xx}++)\{$

if $(\mathrm{xx}==\mathrm{ncol}$ old $) \mathrm{xx}=0$;

if (solid $[z][$ index $[x])$ rho = PH_FSOLID;

else \{

rho $=u$ u $[z]$ [index] $[\mathrm{xx}]$.usqrd;

if (rho>=rhoMax) rhoMax = rho;

if (rho<=rhomin) rhomin = rho; \}

*(pfBuftioffs+x) =rho;

\}

break; / * END 1111111111111111111111111111111111111111111111* /

case $2: I^{*} \mathrm{yz}$ slice 222222222222222222222222222222222222222222222 */

iindex = index of ncol_old;

for $\left(\mathrm{y}=0 ; \mathrm{y}<\right.$ nrow $\left.; \mathrm{y}^{++}\right)\{$

iOffs $=\mathrm{Y} * \mathrm{bCOl}$;

for $(z=0 ; z<$ nlay; $z++)\{$

if (solid $[z][y][$ index $])$ rho = PH_FSOLID;

else \{

rho $=u u[z][y][i$ index] .usqrd;

if (rhos=rhomax) rhomax = rho;

if (rho<=rhomin) rhomin = rho; \}

*(pfBuf+ioffs $+z)=$ rho;

\}

\}

break; /* END 2222222222222222222222222222222222222222222222*/ 


\section{Listing 2. plothead.h}

1* plothead.h -- add $\mathrm{PH}$ _ below to cut down possible conflicts in future, and */

$1 *$ set a value to denote the solid */

\#define PH_MAXTRY 10

\#define PH_NRESERVED 16

\#define PH_USOLID $255 /$ * for unsigned char plotfile, sizeofDatum $=1$ */

\#define PH_FSOLID -999999.0f $/$ * for float plotfile, sizeOfDatum $=4$ *

1 * for the 1-byte unsigned char data type: * 1

\#define PH_MAX1BYTE 235.0f / allow 256 minus 20 system colors... */

\#define PH_MIN1BYTE $0.0 \mathrm{f}$

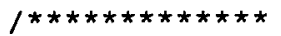

the plot files have following format:

char PH_MACHINE[4]; 4 byte-sized integers:

$\ldots$. 1st is 0 if little-endian like $x 86,1$ if big-endian like most RISC;

$\ldots$ 2nd is sizeof(int) on the system that wrote file;

... 3rd is sizeof(float) on the system that wrote file;

... 4th is 0 (for now);

int step; ...normally 4 -byte

int sizeofPlothead $=$ sizeof (PLOTHEAD); ...normally 4 -byte

struct plothead; ...the structure below

[...data...] ...then the data, according to sliceType type

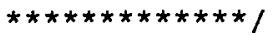

1 * note for $x 86$ every datum is 4 -byte * $/$

struct PLOTHEAD \{

int sliceType; $l^{*} x y=0, x z=1, y z=2, x y z=3 * /$

int iComponent; $/ *$ component number *

int iCol; $\quad l^{*}$ if sliceType $==0,1,3$ this in ncol; if sliceType==2, this is $x$ of slice *

int iRow; $\quad l^{*}$ if sliceType $==0,2,3$ this in nrow; if sliceType= $=1$, this is $y$ of slice *

int ilay; $\quad \quad^{*}$ if sliceType $==1,2,3$ this in nlay; if sliceType= $=0$, this is $z$ of slice $* /$

float ux; $l^{*}$...then the ux, uy, uz speed averages at the speed control slices *

Eloat uy;

float uz;

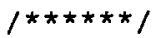

float $f x ; \quad l^{*}$...then the $x, y, z$ fluxes through the flux measurement slices */

float fy;

float fz;

$1 * \star \star \star \star \star * /$

float minDatum; $/ *$ minimum datum written to file, converted to float */

float maxDatum; $/ *$ maxiumum datum written to file, converted to float */

float cofmin; $/ *$ the ff[] value corresponding to minimum datum (useful if 1-byte ints

written) */

float cofMax; /* the ff[] value corresponding to maximum datum (*n" " ") */

float reserved[PH_NRESERVED]; $/$ * for future values; currently garbage */

int sizeofDatum; 1 * size of each item (bytes) in data to follow */

\};

1 * add: for $x 86$ * $/$

static char PH_MACHINE [4] $=\{0,4,4,0\}$; 


\section{Listing 3. pix_test.c}

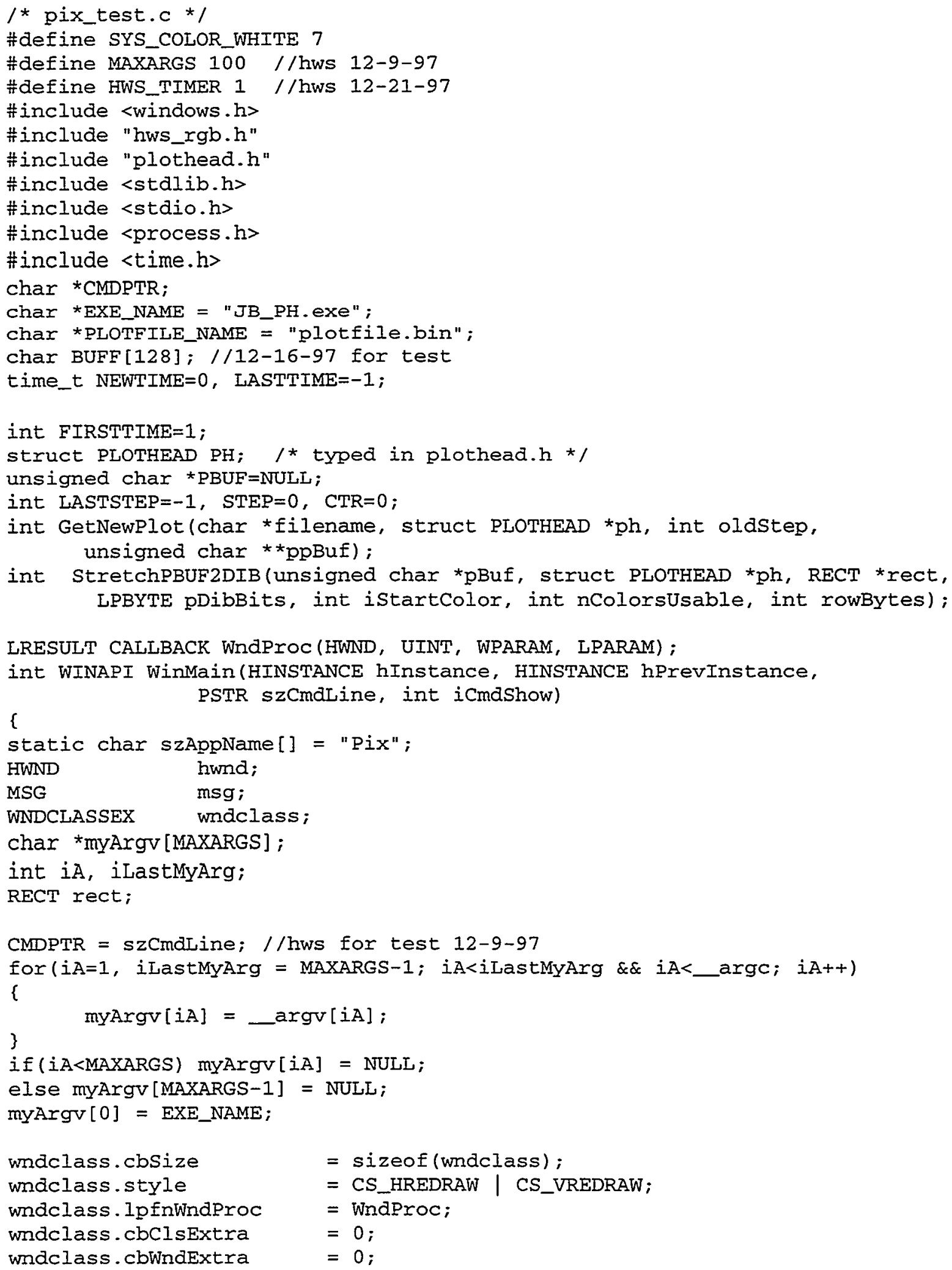




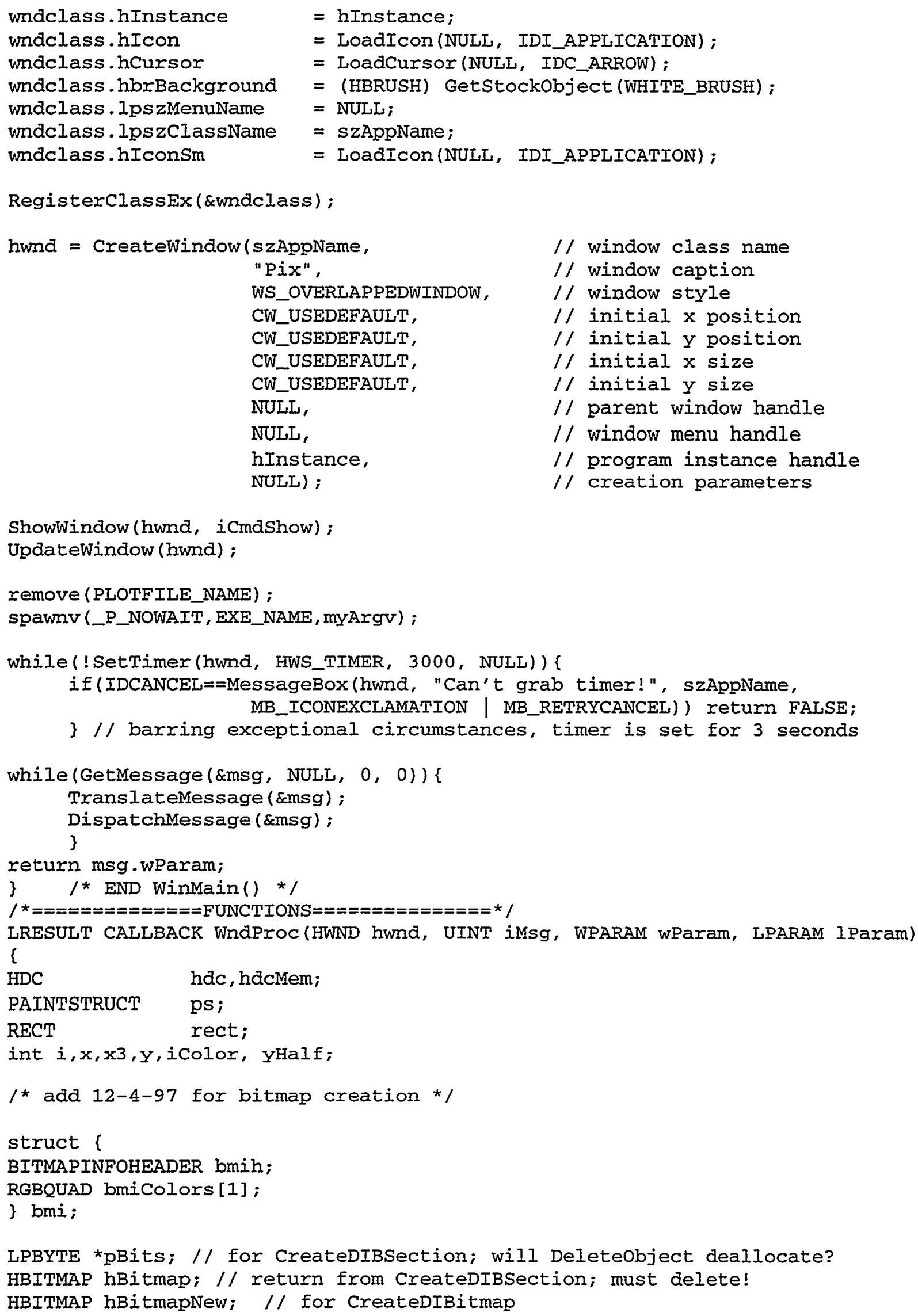

Showwindow (hwnd, iCmashow) ;

Updatewindow (hwnd) ;

remove (PLOTFILE_NAME);

spawnv (_P_NOWAIT, EXE_NAME, myArgv) ;

while(!SetTimer (hwnd, HWS_TIMER, 3000, NULL)) \{ if (IDCANCEL==MessageBox (hwnd, "Can't grab timer!", szAppName, MB_ICONEXCLAMATION | MB_RETRYCANCEL)) return FALSE;

\} // barxing exceptional circumstances, timer is set for 3 seconds 
int ioffs,ii; // account for 3 bytes/row and dword boundary

int ilines;

static int rowBytes;

int $x$ PixperMeter, $y$ PixPerMeter;

LPBITMAPINFOHEADER PDib;

LPBYTE pDibBits;

HDC hdcRef;

HBITMAP hBitmapDIB, hbmold;

HDC hdcBitmap;

RGBQUAD * rgbPtr;

int nColors; / / hws

int istartColor $=10$, nColorsUsable; //hws 12-17-97

switch (iMsg) \{

case WM_CREATE:

return (0);

case WM_TIMER:

STEP = GetNewPIot (PLOTFILE_NAME, \&PH, LASTSTEP, \&PBUF);

if ( STEP>IAASTSTEP \&\& PBUF!=NULL) \{

FIRSTTIME $=0$;

LASTSTEP=STEP;

CTR++;

InvalidateRect (hwnd, NULL, FALSE);

\}

return $(0)$;

case WM_PAINT:

hdc = BeginPaint (hwnd, \&ps);

GetClientRect (hwnd, \&rect);

if (FIRSTTIME) \{

DrawText (hdc, "Waiting for LB data", -1, \&rect, DT_SINGLELINE | DT_CENTER | DT_VCENTER);

EndPaint (hwnd, \&ps); return (0);

\}

// hws should make the 256 more general below

pDib = (LPBITMAPINFOHEADER) GlobalAlloc (GPTR, (sizeof (BITMAPINFOHEADER) + 256 * sizeof(RGBQUAD)));

pDib->bisize = sizeof (BITMAPINFOHEADER);

pDib->biWidth = rect.right;

pDib->biHeight $=$ rect .bottom;

pDib->biPlanes $=1$;

pDib->biBitCount $=8$;

$/ /$ below hws presumes biBitcount $=8$ !

rowBytes $=($ DDib->biWidth +3$)$ \& 0xFFFC;

nColors $=1 \ll$ pDib->biBitcount; $/ /$ hws for future

nColorsusable $=$ nColors $-20 ; / /$ hws 12-17-97

hdCRef $=\operatorname{GetDC}(0)$;

hBitmapDIB $=$ CreateDIBSection (hdcRef, (BITMAPINFO *) pDib,DIB_RGB_COLORS, (VOID **) \&pDibBits, NULL, 0);

hdcBitmap = CreateCompatibleDC (hdcRef);

rgbPtr $=($ RGBQUAD $*)($ pDib +1$) ; / /$ hws

GetSystemPaletteEntries (hdcRef, 0, 10,rgbPtr); 
//hws -- add in some more colors

// 12-15-97 try other palette

for $(i=10, i i=0 ; i<n$ Colors $-10 ; i++, i i++)\{$

$($ rgbPtr+i) $\rightarrow$ rgbBlue $=$ rgbHWSO $[i i]$. rgbBlue;

(rgbPtr+i)->rgbGreen = rgbHWSO [ii].rgbGreen;

(rgbPtr+i) $\rightarrow$ rgbRed = rgbHWSO [ii].rgbRed;

$($ rgbPtr $+i) \rightarrow$ rgbReserved $=0$;

\}

ReleaseDC (0, hacRef);

hbmold = Selectobject (hdcBitmap, hBitmapDIB);

SetDIBColorTable (hdcBitmap, 0 , nColors, (RGBQUAD *) (pDib + 1));

StretchPBUF2DIB (PBUF, \&PH, \&rect,

pDibBits, istartColor, nColorsusable, rowBytes);

$/ /$ hdc $=$ GetDC (hwnd);

BitBlt (hdc, 0,0,pDib->biWidth,pDib->biHeight, hdcBitmap, 0, 0, SRCCOPY) ;

//ReleaseDC (hwnd, hdc);

// 12-16-97 test

sprintf (BUFF, "STEP=ơd, CTR=ơd", STEP, CTR);

DrawText (hdc, BUFF, -1 , \&rect,

DT_SINGLELINE | DT_CENTER | DT_VCENTER);

Selectobject (hdcBitmap, hbmold);

Deleteobject (hBitmapDIB);

Deleteobject (hbmold);

GlobalFree (pDib) ;

Globalfree (pDibBits);

EndPaint (hwnd, \&ps) ;

return (0);

case WM_DESTROY:

PostQuitMessage $(0)$;

return $(0)$;

3 / * END big message switch */

return DefWindowProc (hwnd, iMsg, wParam, lParam);

$3 \quad 1 *$ END WndProc ()$* 1$

$1 * \ldots+\ldots$

/* Routines to interpret PBUF and map to pDibBits; for now */

/* we'll just handle sizeofDatum $=1 \ldots$ * $/$

int stretchPBUF2DIB (unsigned char *pBuf, struct PLOTHEAD *ph, RECT *rect,

LPBYTE pDibBits, int istartColor, int nColorsusable, int rowBytes) (

int $x, y$, ioffs, iColor, $x p, y p ; ~ / *$ latter are into the pBuf * $/$

int ipRow, ipCol, iplay;

int ipoffs;

float xMul, yMul, cMul, cDelta;

unsigned char cVal;

if $(\mathrm{ph} \rightarrow>$ sizeofDatum $>$ 1) return $(0) ; / *$ change when we have float handler *

I* No matter what, need to know the correspondence of iRow, iCol and */

/* ilay stored in new file with logical coordinates in pBuf... */

/* From sliceType, determine the mapping of file rows into logical */

1 * screen rows... fisrt do the setup: */ 


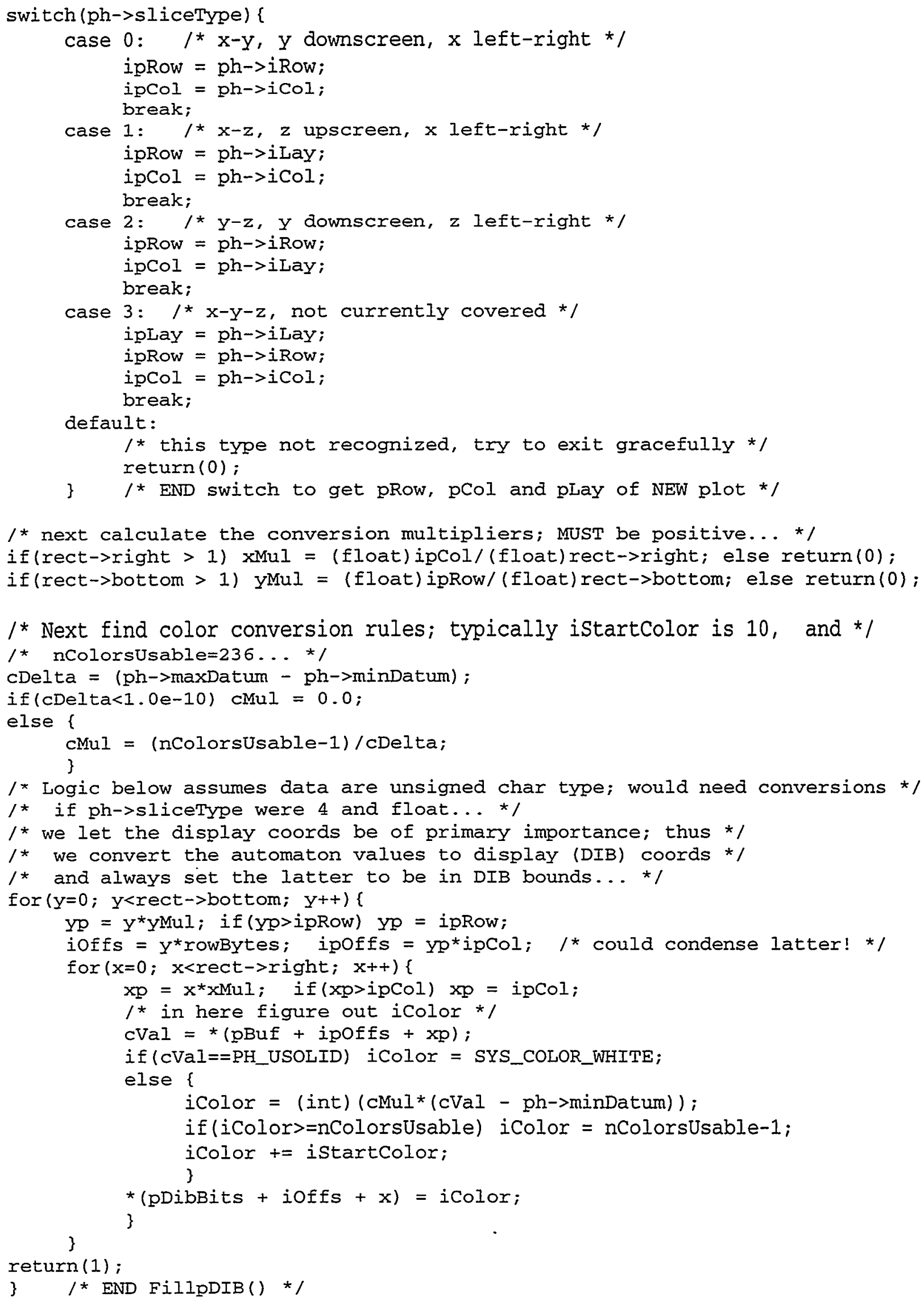




\section{Listing 4. GetNewP1ot()}

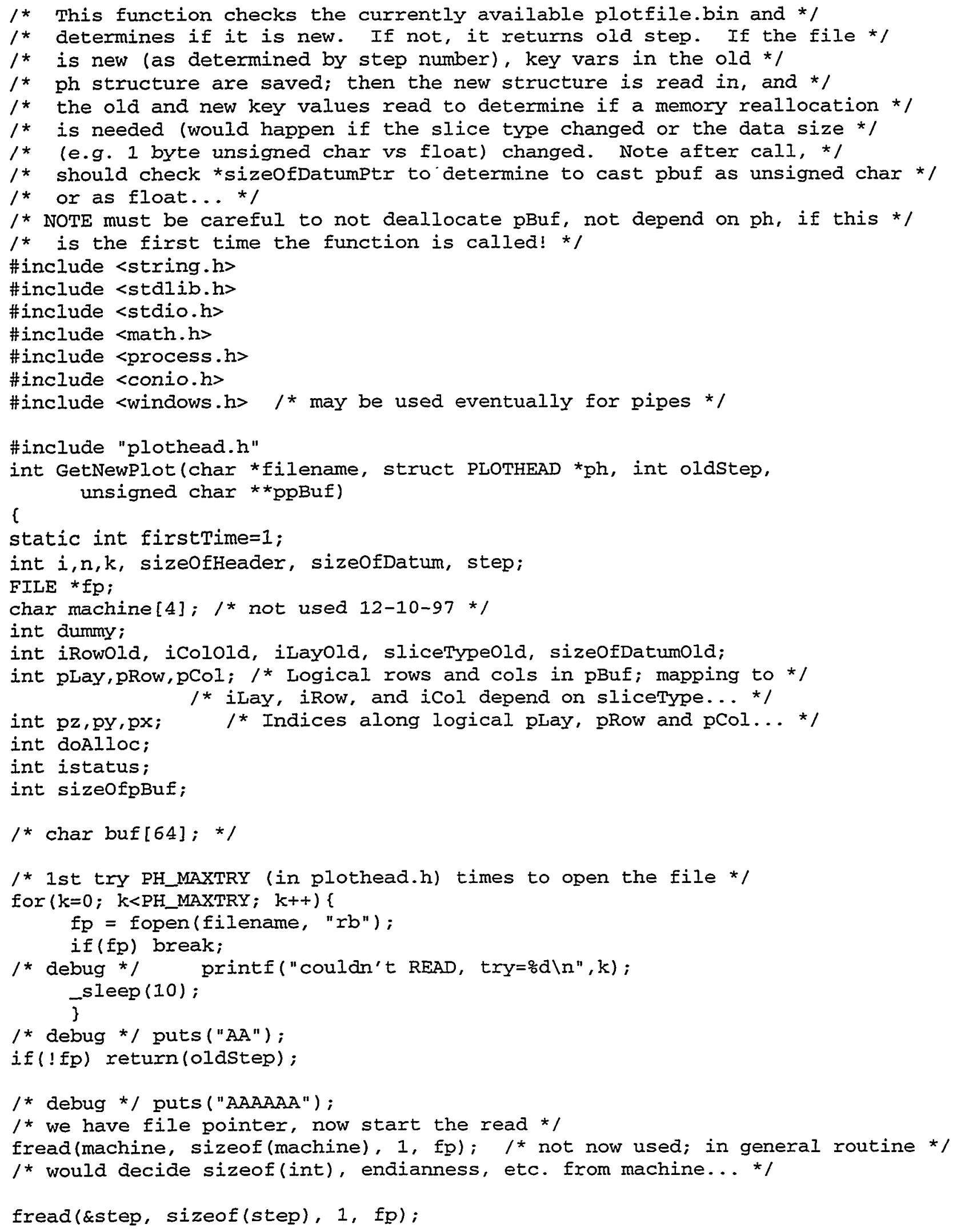




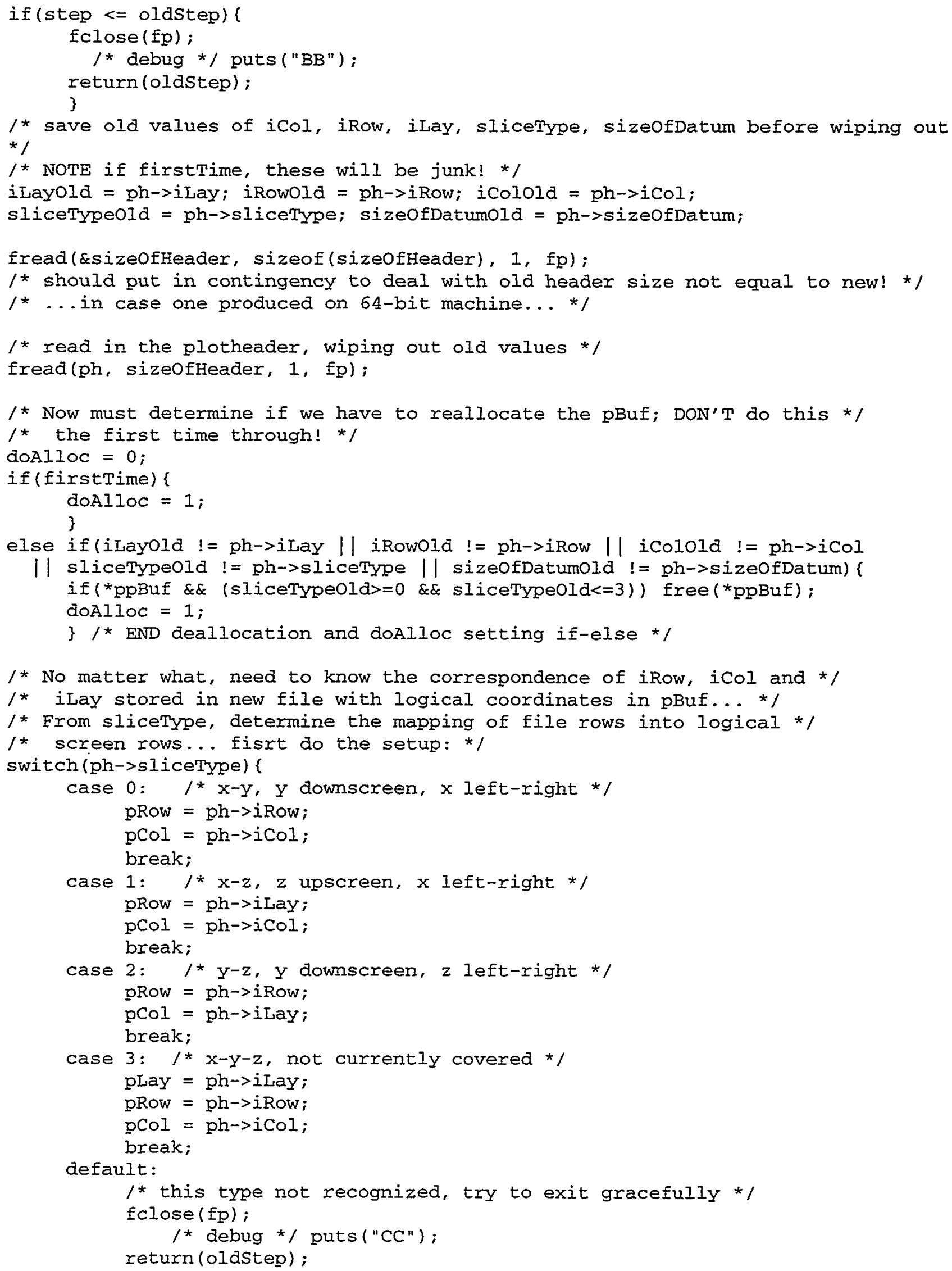


3 /* END switch to get pRow, pCol and pLay of NEW plot */

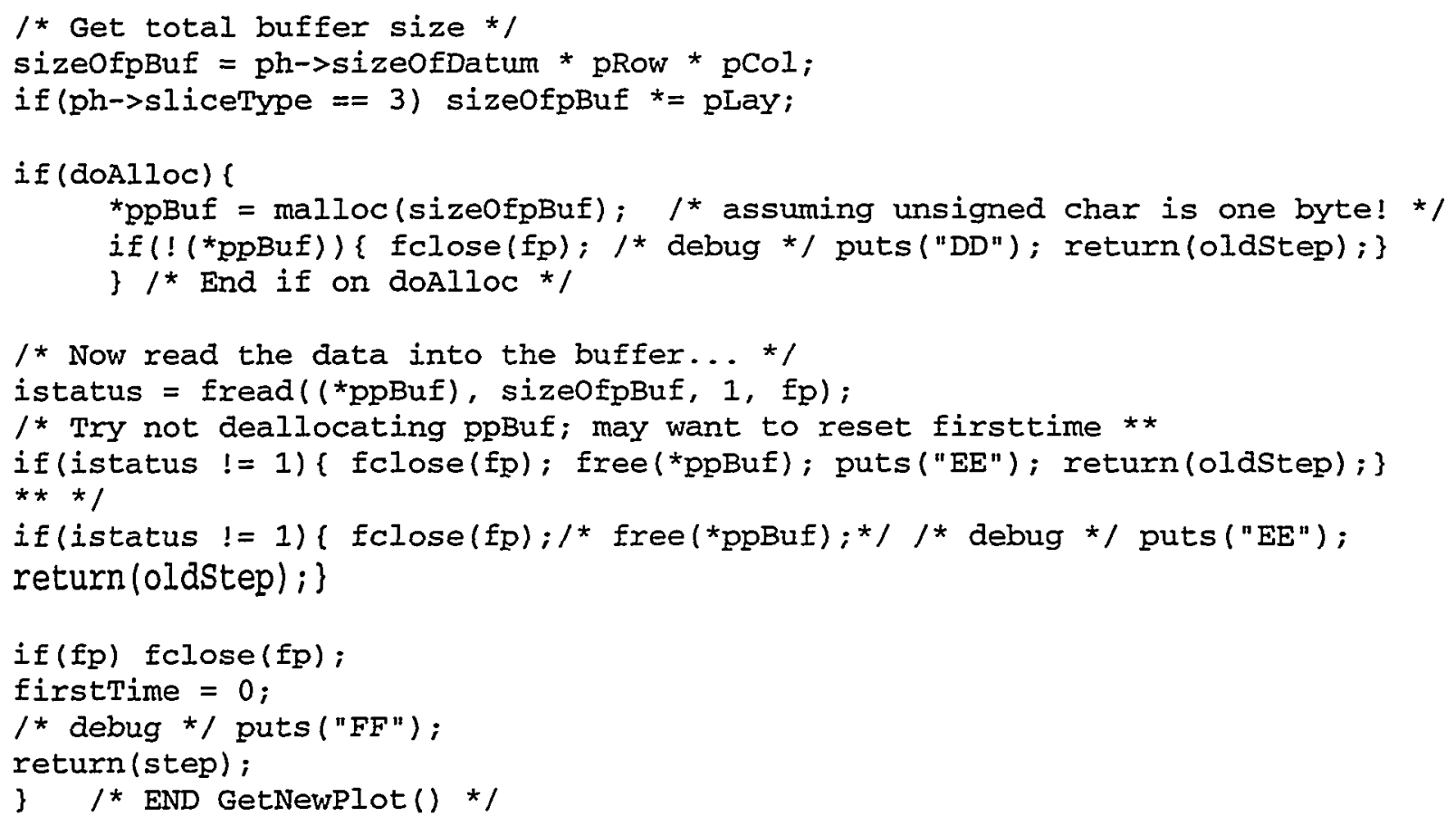




\section{Listing 5. JB_PH.C}

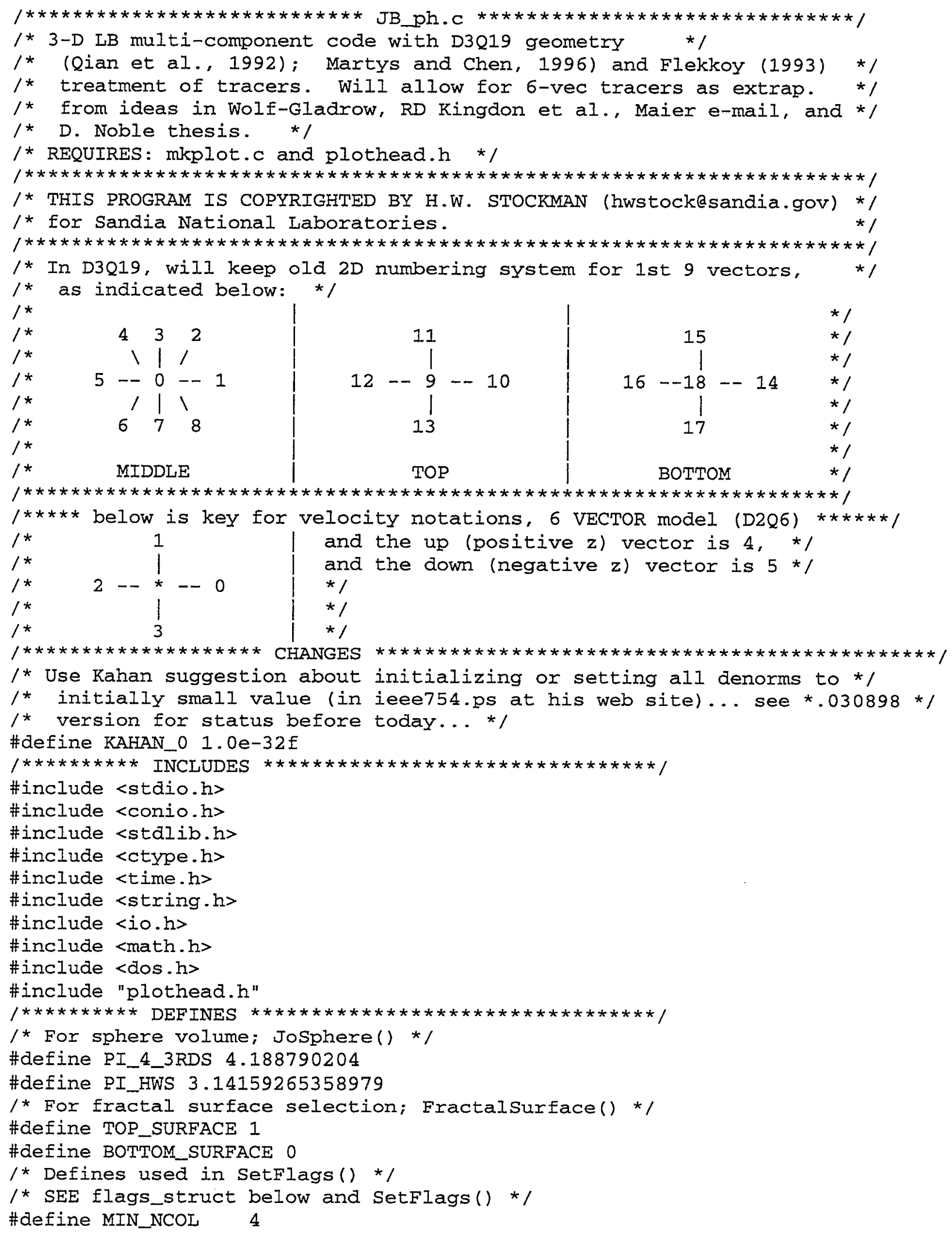




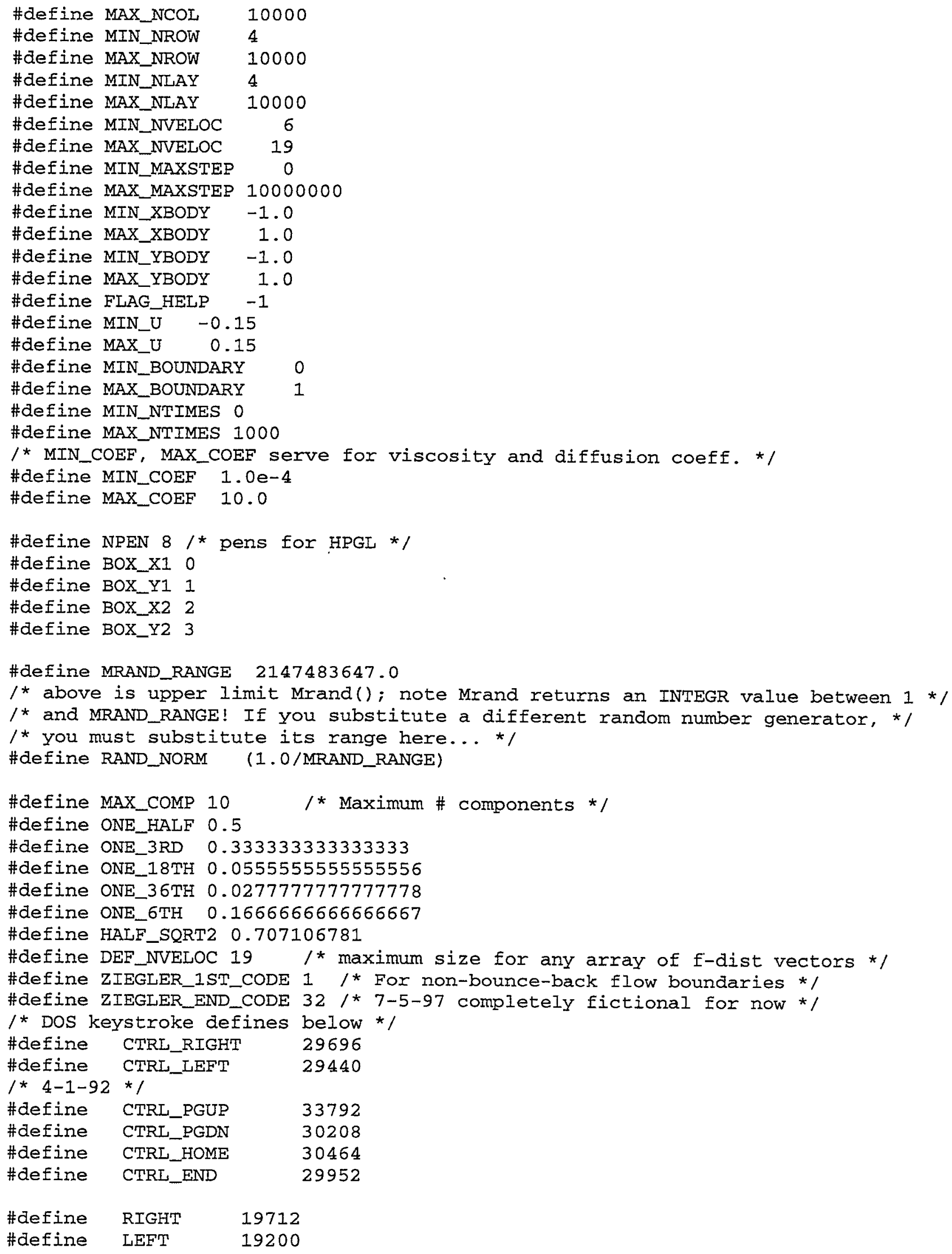




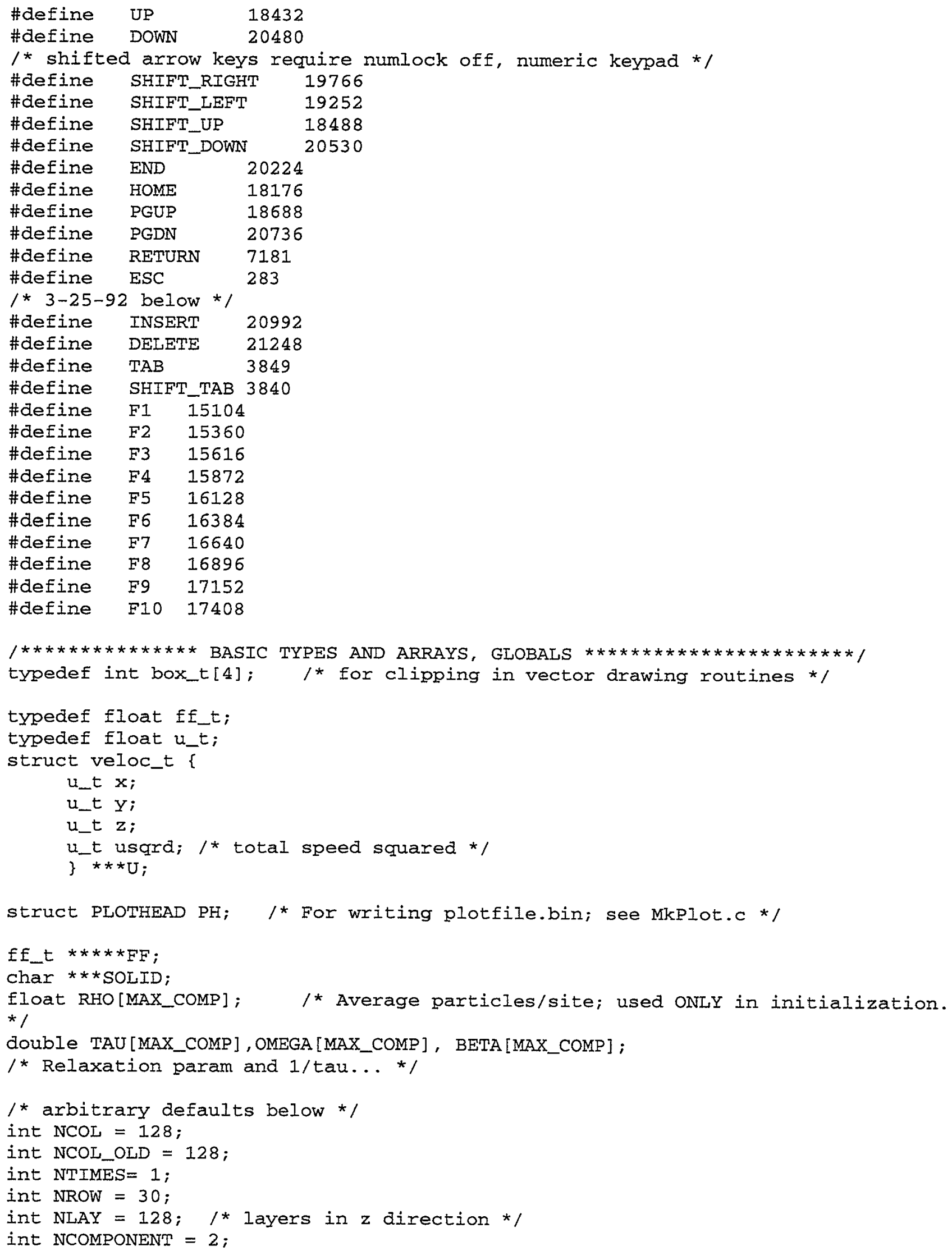


int NVELOC [MAX_COMP] $=\{19,6,6,6,6,6,6,6,6,6\} ; /$ * for now, set all tracers $=6 * /$ int STEP $=0$, MAXSTEP $=10000$;

int RADIUS $=0 ; 1 * 10-29-97$ sphere radius for jo.c */

int JO_AND_STEP $=31 ; 1$ * 10-29-97 for jo.c only */

int JO_SOLIDS $=0 ; /$ * 10-29-97 for jo.c only * $/$

int USE_ALVEOLI $=0, X Z A L V=2, Y A L V=1$;

int $\mathrm{XSCR}=79, \mathrm{YSCR}=24 ; / * \max$ number rows, columns in screen *

int SOLIDS_SET $=0$;

int SOLIDS_ENCLOSED $=0$;

int JUST_DISPERSE_STEP $=$ MAX_MAXSTEP;

1 * List of $z$ values to pass to SaveUPpm(), SaveVect () */

\#define NZ 4

int $\mathrm{ZZ}[\mathrm{NZ}]$;

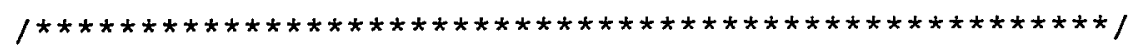

$1 *$ Set up an array for ideal equil distribution in a 19-vector */

/* carrier fluid... */

double TT $[19]=$

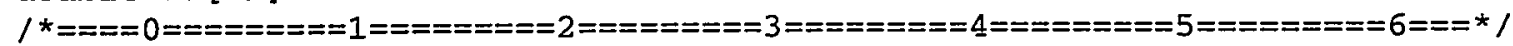

$\{$ ONE_3RD , ONE_18TH, ONE_36TH, ONE_18TH, ONE_36TH, ONE_18TH, ONE_36TH,

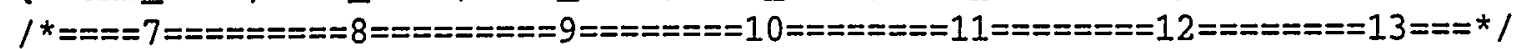

ONE_18TH, ONE_36TH, ONE_18TH，ONE_36TH，ONE_36TH，ONE_36TH，ONE_36TH，

$l^{*}===14========15========16========17=========18====*$ /

ONE_36TH, ONE_36TH, ONE_36TH, ONE_36TH, ONE_18TH\};

1* Arrays for coord deltas in $x, y, z$ directions, corresponding */

$1 *$ to the notation at top of file; should ease logic in functions */

$1 *$ and should eventually be incorporated in an */

/* include file (*.h)... Given to help other programers; currently unused. */

int $\mathrm{DX}[19]=$

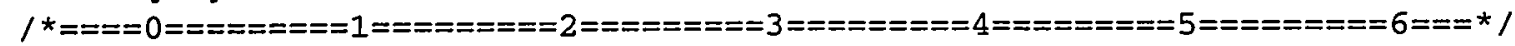

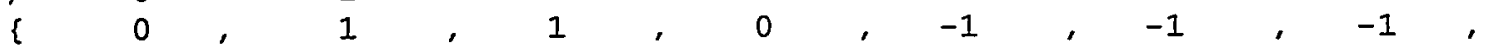

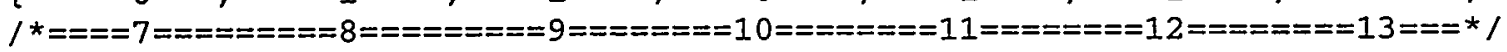

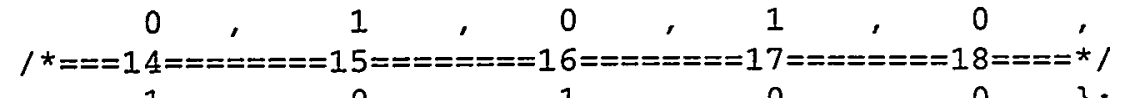

int $D Y[19]=$

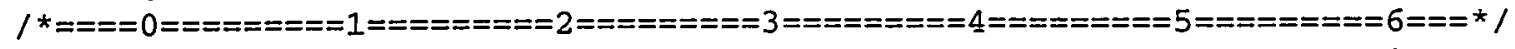

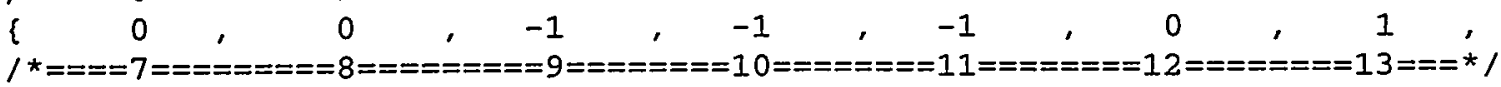

$1,1,0,0,-1,0,1$,

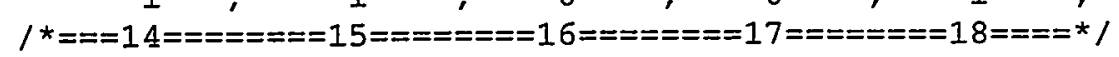

$0,-1,0,1,0$;

int $\mathrm{DZ}[19]=$

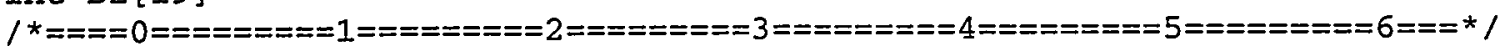

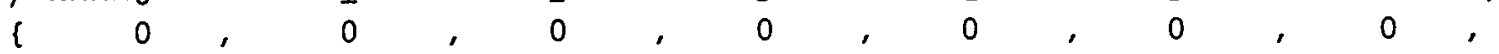

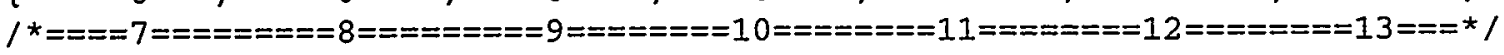

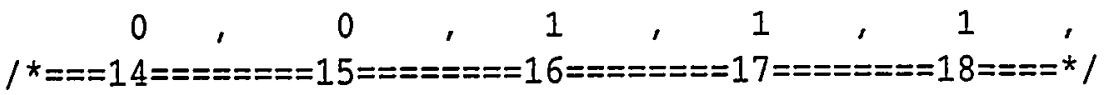

$-1,-1,-1,-1,-1\}$;

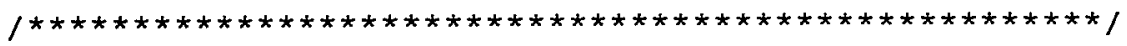




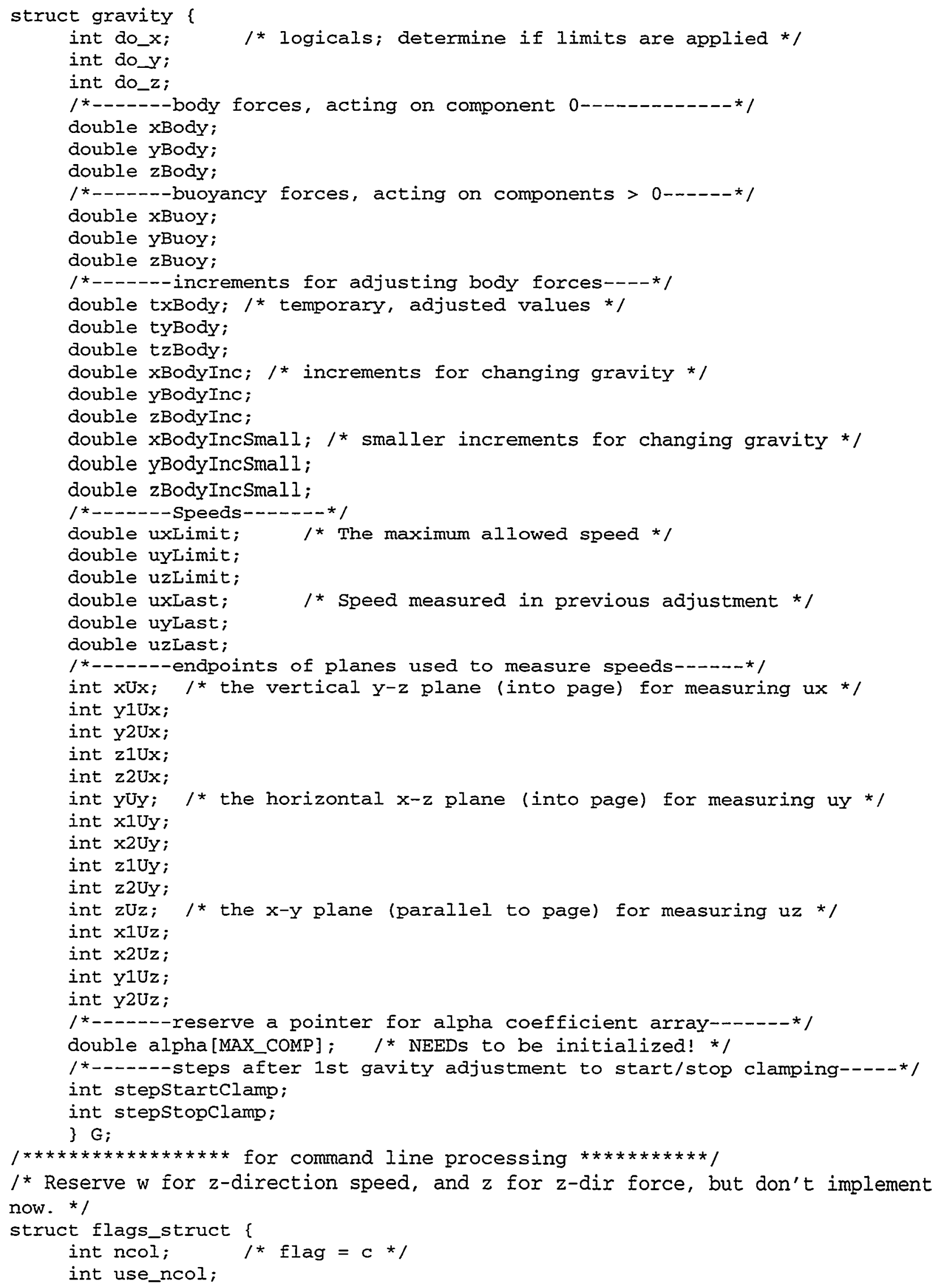




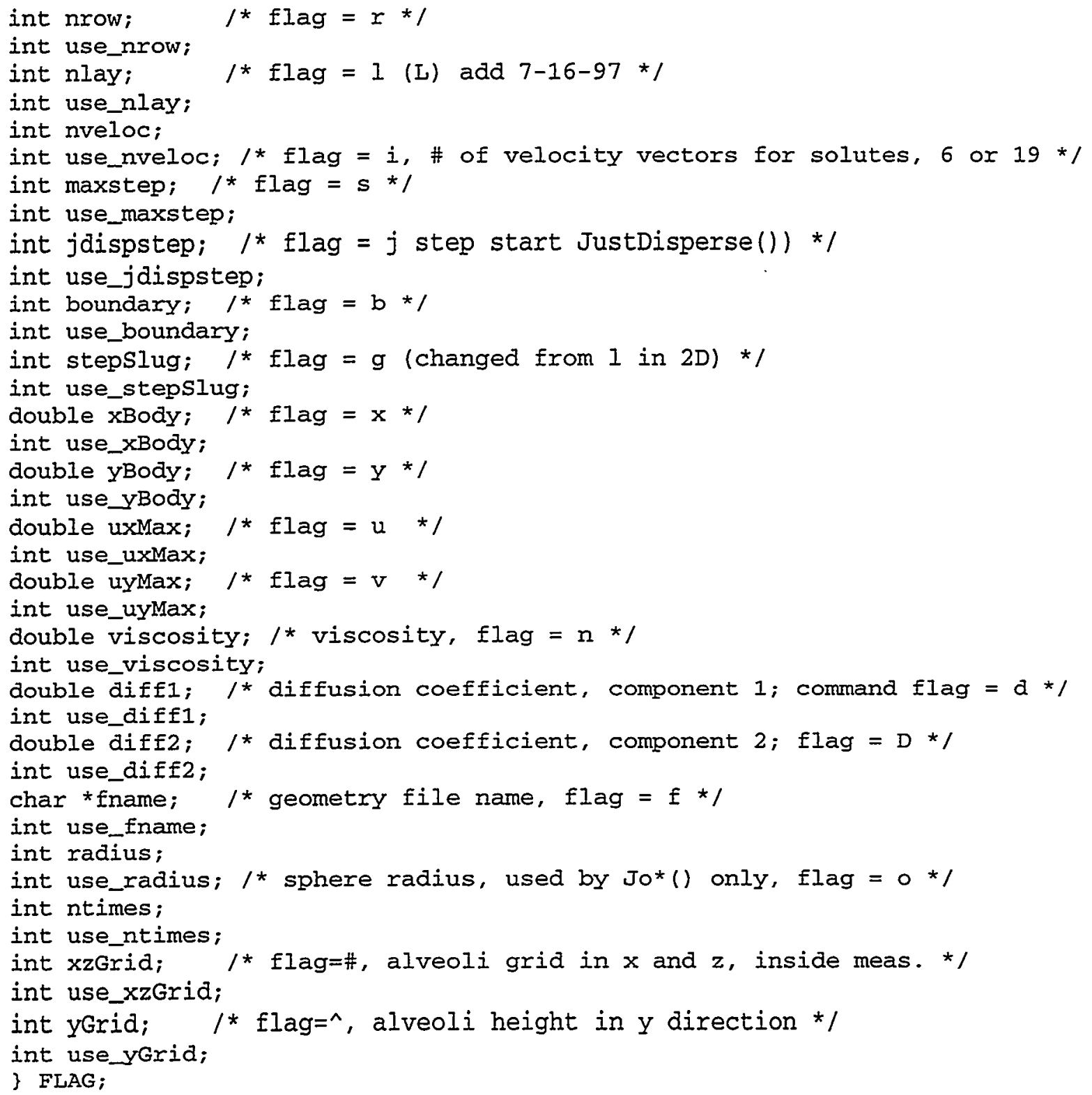


struct gravity * const gp, char *const*const* solid,

int ncomponent, int nveloc[], int nlay, int nrow, int ncol),

Plot9Vec(ff_t $* * * * *$ const ff, float * const rho, int ncomponent, int nveloc[], int nlay, int nrow, int ncol, int step),

PlotConcs (Ef_t *const*const*const*const* ff, char * const* const* solid, float * const rho, struct veloc_t *const*const* uu, int ncomponent, int nveloc[], int nlay, int nrow, int ncol, int ncol_old, $/$ * 10-30-97 add ncol_old */

int step, int $s$, int xStart, int ystart, int $z z$, int zoomMode),

SetSlug(ff_t $* * * * * f f$, char $* * *$ solid, int ncomponent, int nveloc[], int nlay, int nrow, int ncol, int $x 1$, int $x 2$, int $y 1$, int $y^{2}$, int $z 1$, int $z 2$, double * conclist),

SetSolidType(char ***solid, int nlay, int nrow, int ncol),

Mrand (int z),

SetFCCSolids(char $* * *$ solid, int nlay, int nrow, int ncol, int zrep, int yrep, int xrep, float rslop, float cslop, float rbias, int seed),

AdjustGrav(struct veloc_t *const*const* uu, char *const*const* solid,

int nlay, int nrow, int ncol, struct gravity * const gp),

Xsetconclff_t $* * * * *$ const ff, char $* * *$ const solid, int ncomponent, int nveloc[], int nlay, int nrow, int ncol, int $x$, int $y 1$, int $y^{2}$, int $z 1$, int $z 2$, double * const conclist),

YSetConc(ff_t $* * * * *$ const ff, char $* * *$ const solid, int ncomponent, int nveloc[], int nlay, int nrow, int ncol, int $y$, int $x 1$, int $x 2$, int $z 1$, int $z 2$, double * const conclist),

SaveUPpm (struct veloc_t *** const uu, char ***solid, int nlay, int nrow, int ncol, int $z z[]$, int $n z$, int step),

SaveVect (struct veloc_t $* * *$ const uu, char $* * *$ solid, int nlay, int nrow, int ncol, int $z z[]$, int nz, int step, int nxavg, int nyavg, int uselog, int usecolor, float maxvectFrac),

Clipline (float *x1p, float *y1p, float *x $x$, float ${ }^{*} y 2 p$, box_t box),

Clipt (double $p$, double $q$, double *t1p, double *t $2 p$ ),

DrawBox(float $x 1$, float $x 2$, float $y 1$, float $y^{2}$, box_t clipbox, FILE *fp, int color),

SetFlags (int argc, char *argv[], struct flags_struct *fp),

Fractalsurface (char $* * *$ const solid, int nlay, int nrow, int ncol, int hpix, char *fname, int toporBottom, int offs), 
JustDisperse3D (ff_t ${ }^{*}$ const* const* const* const* ff, double * const tau, double * const beta, double *const omega, struct veloc_t * const*const* uu, char *const*const* solid, int ncomponent, int nveloc[], int nlay, int nrow, int ncol, int ncol_old),

/* Next two: add capability to select simpler function */

JustDisperse3D_A(ff_t * const* const*const*const* ff, double * const tau, double * const beta, double *const omega, struct veloc_t *const*const* uu, char *const*const* solid, int ncomponent, int nveloc[], int nlay, int nrow, int ncol, int ncol_old),

(*JustDisp_ptr) (ff_t *const*const*const*const* ff, double * const tau, double * const beta, double *const omega, struct veloc_t *const*const* uu, char *const*const* solid, int ncomponent, int nveloc[], int nlay, int nrow, int ncol, int ncol_old),

ReadBmp4Slug(ff_t $* * * * *$ const ff, char $* * *$ const solid, float rho, int nvel, int nlay, int nrow, int ncol, int sstart, int $x$, int $z$, char *filename),

YSetConcJB(ff_t $* * * * *$ const ff, char $* * *$ const solid, int ncomponent, int nveloc[], int nlay, int nrow, int ncol, int $y$, int $x 1$, int $x 2$, int $z 1$, int $z 2$, double * const conclist),

XSetConcJB(ff_t $* * * * *$ const ff, char $* * *$ const solid, int ncomponent, int nveloc[], int nlay, int nrow, int ncol, int $x$, int $y 1$, int $y_{2}$, int $z 1$, int $z 2$, double * const conclist),

Recycle(ff_t $* * * * *$ const ff, char $* * *$ const solid, int ncomponent, int nveloc[], int nlay, int nrow, int ncol, int ntimes, int xRight),

JoSphere(char ***solid, int nlay, int nrow, int ncol, int radius),

/* For Eidsath et al. (1983) test */

JoDisk(char $* * *$ solid, int nlay, int nrow, int ncol, int radius),

MakePlot(ff_t $* * * * *$ const ff, struct veloc_t $* * *$ const uu, char $* * *$ const solid, int nveloc [], int ncomponent, int nlay, int nrow, int ncol, int ncol_old, int step, struct PLOTHEAD *ph, int sliceType, int sizeofDatum, int iComponent, int index);

/* The 1st three are dumy placeholders for non-bounce-back walls. */

double NoFlux (double $f[]$, int solidcode, int mode), NoFlux6(double $f[]$, int solidcode, int mode), ziegler (double $f[]$, int solidcode, int mode),

UxAvg (struct veloc_t ${ }^{*}$ const* const* uu, char * const* const* solid, int nlay, int nrow, int ncol, int $x$, int $y 1$, int $y 2$, int $z 1$, int $z 2$ ),

UyAvg (struct veloc_t * const*const* uu, char * const* const* solid,

int nlay, int nrow, int ncol, int $y$, int $x 1$, int $x 2$, int $z 1$, int $z 2$ ),

UzAvg (struct veloc_t * const*const* uu, char *const* const* solid,

int nlay, int nrow, int ncol, int $z$, int $x 1$, int $x 2$, int $y 1$, int $\left.y_{2}\right)$, 
XGetFlux(ff_t $* * * * *$ const ff, char $* * *$ const solid, int ncomponent, int nveloc[], int nlay, int nrow, int ncol, int $x$, int $y 1$, int $y 2$, int $z 1$, int $z 2$, int $s$, double * const ftotPt $x$,

YGetFlux(ff_t $* * \star * *$ const ff, char $* * *$ const solid, int ncomponent, int nveloc[], int nlay, int nrow, int ncol, int $y$, int $x 1$, int $x 2$, int $z 1$, int $z 2$, int $s$, double * const ftotptr),

DrawArrow(float $x \times 1$, float $y y 1$, float $x \times 2$, float $y y 2$, float factor, float mul, int color, FILE *hpgl_fp, box_t box),

Meanstd(ff_t $* * * * *$ const ff, char $* * *$ const solid, int ncomponent, int nveloc[], int nlay, int nrow, int ncol, int sstart, int step, char *pathname, int use_z);

FILE *SavePpm(ff_t $* \star \star \star \star f f$, char $* * \star$ Solid, int ncomponent, int nveloc[], int nlay, int nrow, int ncol, int sstart, int step),

*Save3DPpm (ff_t $* \star * \star * f f$, char $* * *$ solid, int ncomponent, int nveloc[], int nlay, int nrow, int ncol, int sstart, int step),

*SavePpmXY_XZ(ff_t $* * \star * * f f$, char $* * \star s o l i d$, int ncomponent, int nveloc[], int nlay, int nrow, int ncol, int sstart, int step, int colorCode);

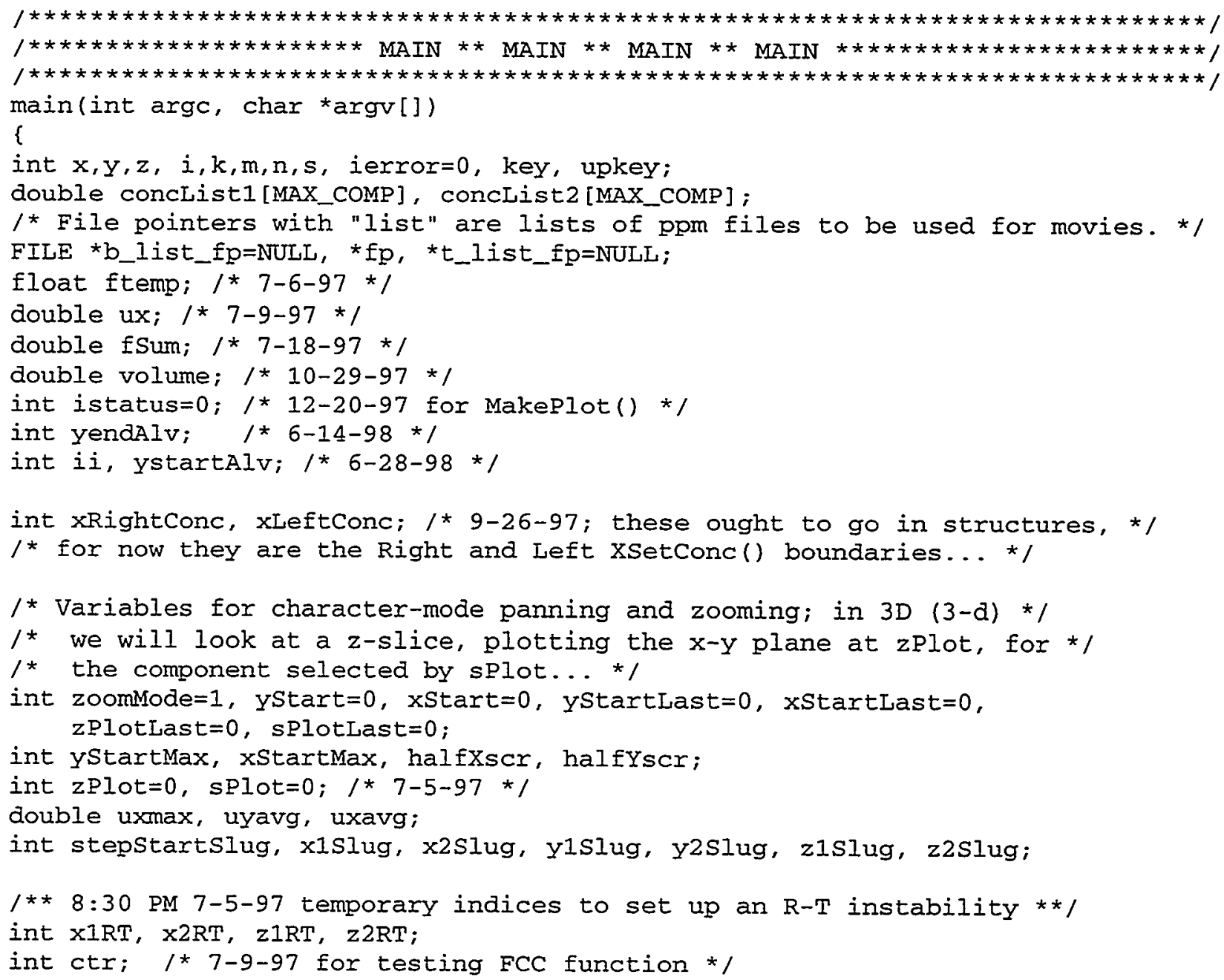




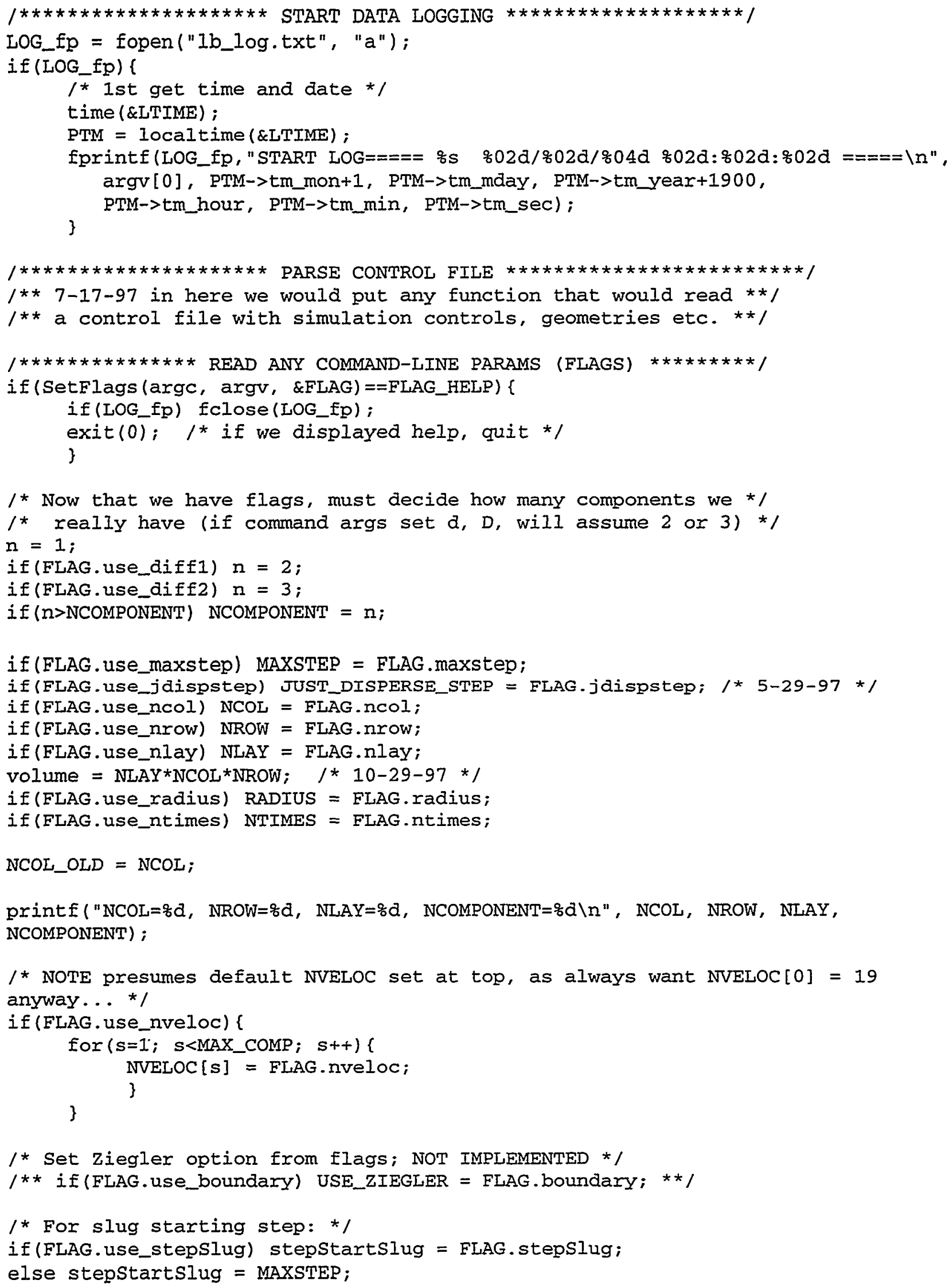


/ 7-18-97 test zero-speed case, so change 1st line below temporarily */

$1 *$ 12-26-97 modify xlslug below to get after 1st sphere-- copied from *

$1 *$ ljb_sphereljb_l.c*/

$1 * * 2-28-98$ comment out sphere-slug for Eidsath et al. Disk test $* * /$

$x$ lslug $=($ NCOL $>>1) ;$

$x 2$ slug $=x 1$ Slug $+($ NCOL $>>2) ;$

$\mathrm{Y} 1$ Slug $=0 ; \mathrm{Y} 2$ Slug $=$ NROW-1;

zISlug $=0 ;$ z2Slug $=$ NLAY $-1 ;$

$1 * * 2-27-98$ end comment out **1

$1 * 6-14-98$ comment out below */

$1 * * 2-28-98$ more appropriate slug for Eiasath et al. test $* *$ $x$ ISlug $=2 *_{\text {RADIUS }-1 ;}$

$\mathrm{x} 2$ slug $=$ NCOL -2 ;

$\mathrm{y} 1 \mathrm{slug}=0 ; \mathrm{y} 2 \mathrm{Slug}=\mathrm{NROW}-1 ;$

z1Slug $=0 ; z 2$ Slug $=$ NLAY $-1 ;$

** 2-28-98 END more appropriate slug for Eidsath et al. test **/

/* 7-11-97 for SaveUPpm(), 7-14-97 Savevect() */

$\mathrm{k}=\mathrm{NLAY} / \mathrm{NZ}$;

for $(i=0 ; i<N Z ; i++) \quad Z Z[i]=k * i$;

/* 2-24-98 KLUDGE for JFM article: */

$\mathrm{ZZ}[1]=1 ; \operatorname{ZZ}[2]=2 ; \mathrm{ZZ}[3]=3$;

/* Set up plotting constraints for character-mode (console) */

/* display via PlotConc()...*/

1 * last xstart before abutting end of ...* $/$

xStartMax = NCOL-1-XSCR; yStartMax = NROW-1-YSCR;

halfXscr $=$ XSCR > $>$; halfYsCr $=$ YSCR $\gg 1$;

sPlot $=0 ; 1 * 7-9-97$ for testing * $/$

1 * primary memory allocation; start over sigmas */

$F F=\operatorname{malloc}\left(\right.$ sizeof $\left(f f \_t * * * *\right) *$ NCOMPONENT);

if(!FF) goto error_primary_alloc;

for $(s=0 ; s<$ NCOMPONENT; $s++)\{1$ * 5-22-97 note NVELOC now array *

$F F[s]=\operatorname{malloc}\left(\right.$ sizeof $\left(f f \_t * * *\right) *$ NVELOC $\left.[s]\right)$;

if(!FF[S]) goto error_comp_alloc;

for $(i=0 ; i<$ NVELOC $[s] ; i++)\{$

$F F[s][i]=\operatorname{malloc}\left(\operatorname{sizeof}\left(f f_{-} t * *\right) *_{\text {NLAY }}\right)$;

if (!FF[s] [i]) goto error_veloc_alloc;

for $(z=0 ; z<N L A Y ; z++)\{$

$F F[s][i][z]=\operatorname{malloc}\left(\right.$ sizeof $\left.\left(f f \_t *\right) * N R O W\right) ;$ if (!FF[s][i][z]) goto error_z_alloc;

for $(\mathrm{y}=0 ; \mathrm{y}<\mathrm{NROW} ; \mathrm{y}++)\{$

$F F[s][i][z][y]=\operatorname{malloc}\left(\operatorname{sizeof}\left(f f_{-} t\right) * \mathrm{NCOL}\right)$;

if(!FF[s][i][z][y]) goto error_row_alloc;

\}

\}

\}

printf("Completed allocations! $\backslash n ") ; / * 5-22-97$ change below */

1 * initialize the FF to 0.0 out of paranoia */

for $(s=0 ; s<$ NCOMPONENT; $s++)\{$

for $(i=0 ; i<\operatorname{NVELOC}[s] ; i++)\{$ 


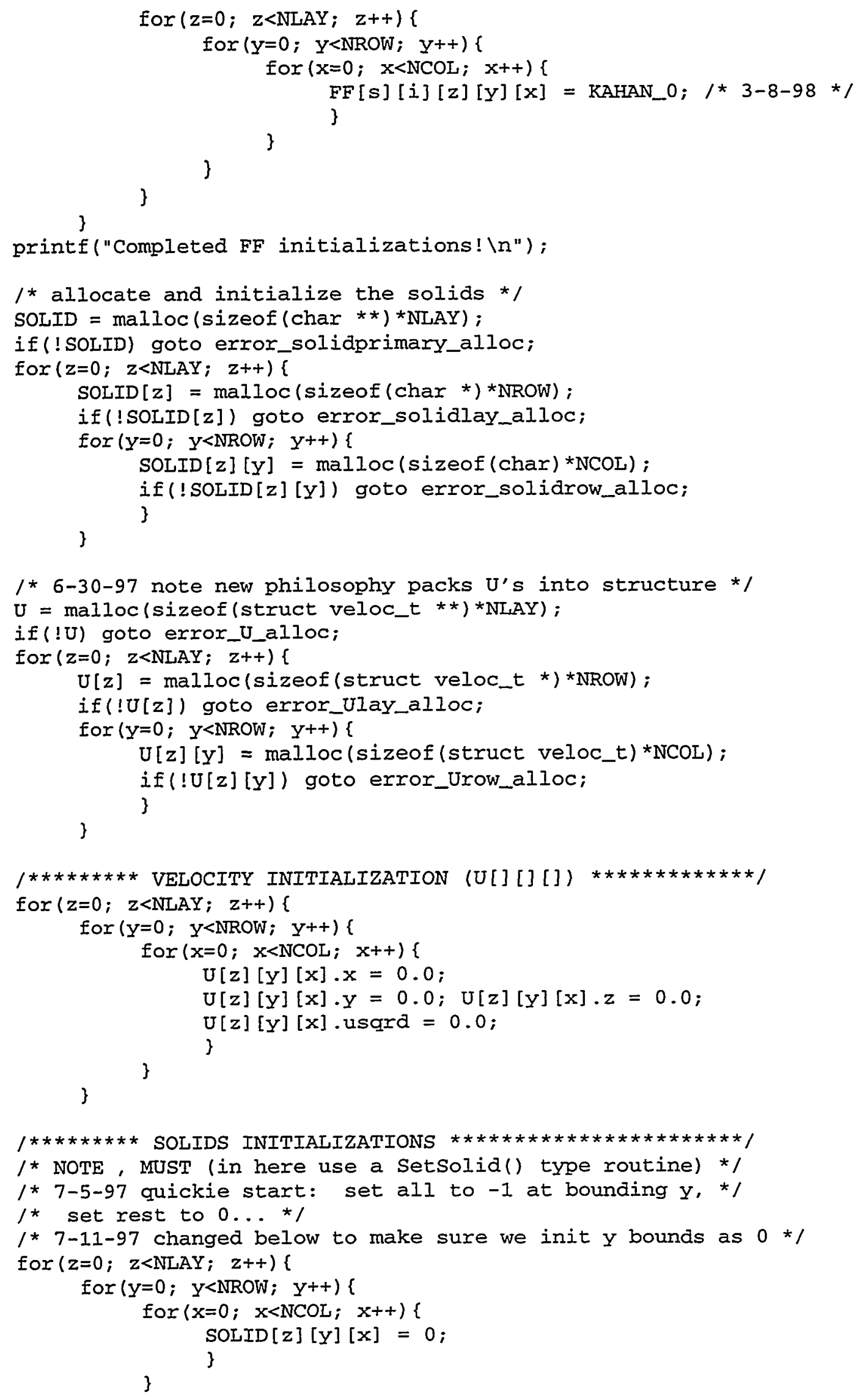




\section{\}}

/ * 8-1-98 bounding planes back in for T-A at other viscosities */

1 * 6-21-98 back in for bounding planes * 1

$/ *$ 10-29-97 comment out bounding solid planes **

for $(z=0 ; z<N L A Y ; z++)\{$

for $(x=0 ; x<N C O L ; x++)\{$

$\operatorname{SOLID}[z][0][x]=-1$;

$\operatorname{SOLID~}[z][N R O W-1][x]=-1 ;$

\}

\}

** end 10-29-97 comment out **/

1 ** 6-23-98 TEMPORARY front wall and top wall for DDG test **

for $(y=0 ; y<N R O W ; y++)\{$

for $(x=0 ; x<N C O L ; x++)\{$ SOLID $[0][y][x]=-1 ;$

)

EOr $(z=0 ; \quad z<$ NLAY; $z++)\{$

for $(x=0 ; x<N C O L ; x++)\{$ $\operatorname{SOLID}[z][0][x]=-1 ;$

\}

** END 6-23-98 TEMPORARY front wall and top wall for DDG test **/

1* 9-17-97 read in steve Brown's fractal geometry files... */

/* for now, just hardwire the names... 9-23-97 hpix from 10 to $16 \ldots$ * /

$1 * *$ 10-29-97 comment out fractal read **

ierror = FractalSurface (SOLID, NLAY, NROW, NCOL, 20, "srb1.txt",1,1);

printf("at Fractal read, ierror $=$ od $\backslash n$ ", ierror);

ierror = Fractalsurface (SOLID, NLAY, NROW, NCOL, 20, "srb2.txt",0,1);

printe("at Fractal read, ierror $=8 d \backslash n "$, ierror);

** end 10-29-97**/

/* 7-9-97 test the FCC algorithm */

$1 * * * * * 7-18-97$ comment out to do Taylor-Aris $* * * * *$

ctr $=$ SetFCCSolids (SOLID, NLAY, NROW, NCOL, 2, 2, 2, 0.20, 0.20, $0.75,178923)$;

printf ("\# spheres $=8 d \backslash \mathrm{n} "$, ctr) ;

$* * \star * *$ END 7-18-97 comment out to do Taylor-Aris $* * * * * /$

$1 * * 2-27-98$ comment out sphere test: $* * /$

if (RADIUS) \{

JO_SOLIDS = JoSphere (SOLID, NLAY, NROW, NCOL, RADIUS);

printf ("** Number of solids is $\% d \backslash n "$, JO_SOLIDS);

printf("*** actual solid/volume is $8 f \backslash n "$, JO_SOLIDS/volume);

printf ("*** predicted solid/volume is $8 f \backslash n "$,

(RADIUS*RADIUS*RADIUS*PI_4_3RDS) /volume) ;

if (LOG_fp) \{

fprintf(LOG_fp, "** Number of solids is sd\n", JO_SOLIDS);

fprintf(LOG_fp, $" * * *$ actual solid/volume is $8 f \backslash n "$, JO_SOLIDS/Volume);

fprintf(LOG_fp,"*** predicted solid/volume is $8 f \backslash n "$, \} (RADIUS*RADIUS*RADIUS*PI_4_3RDS) /volume); 


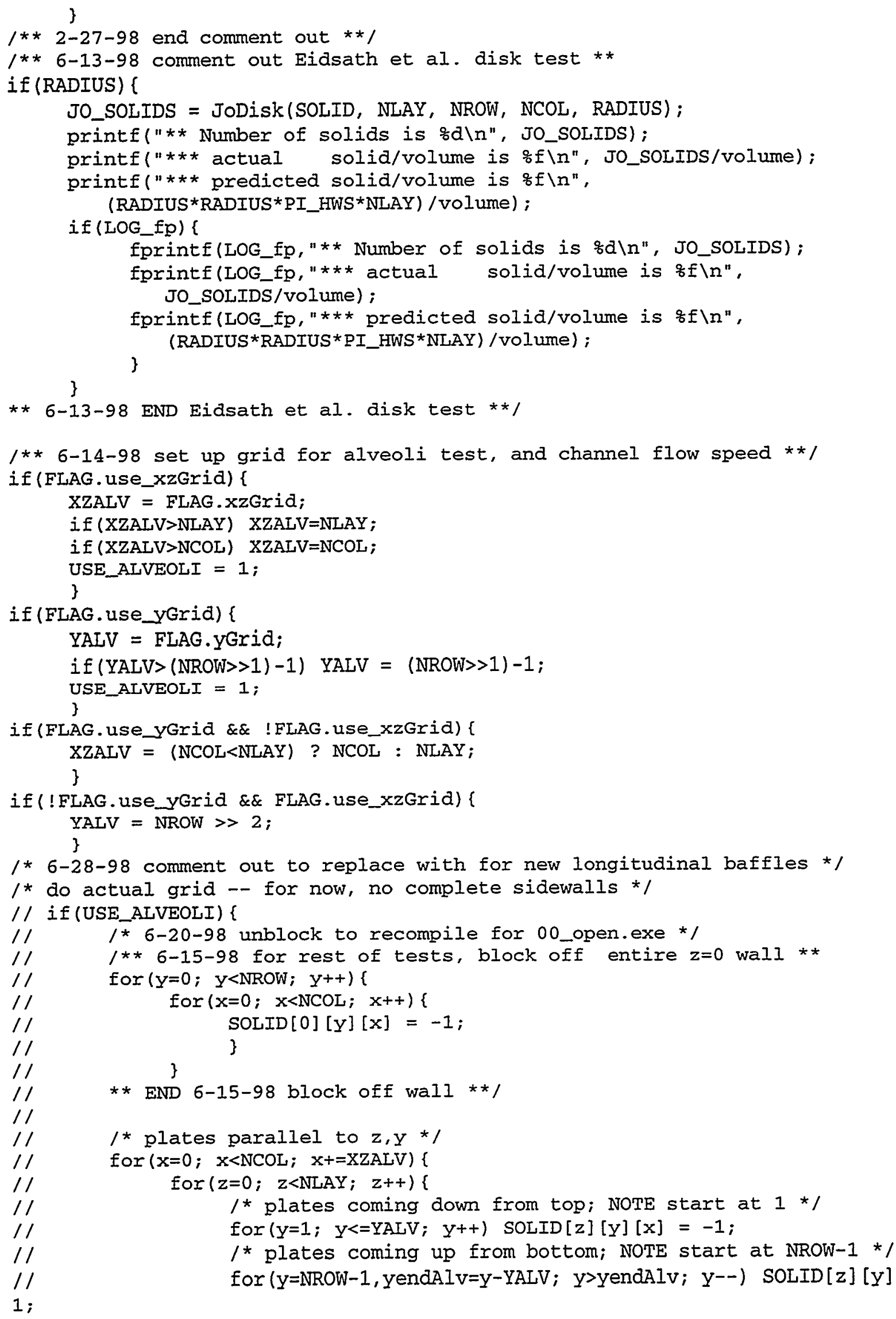




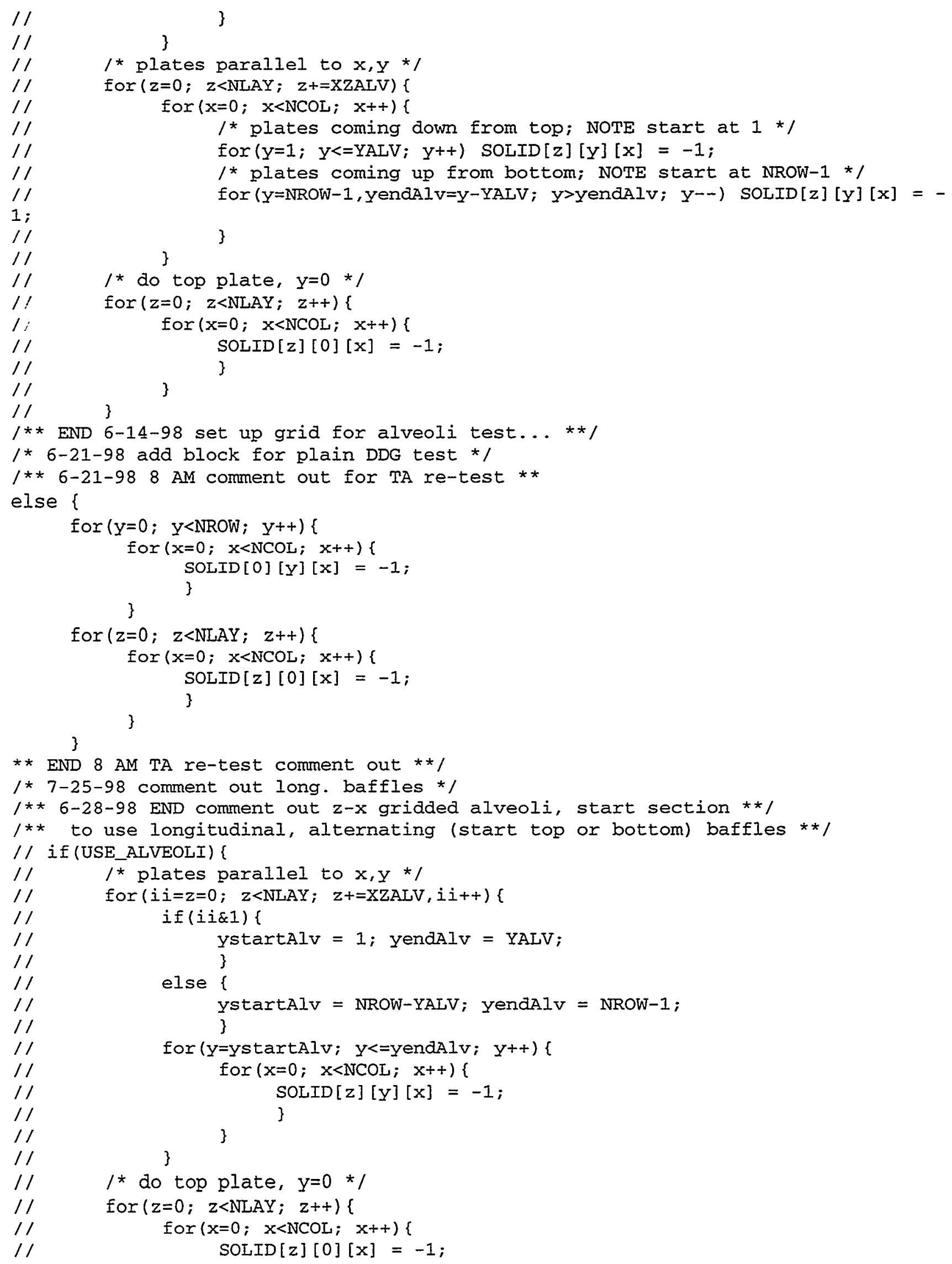




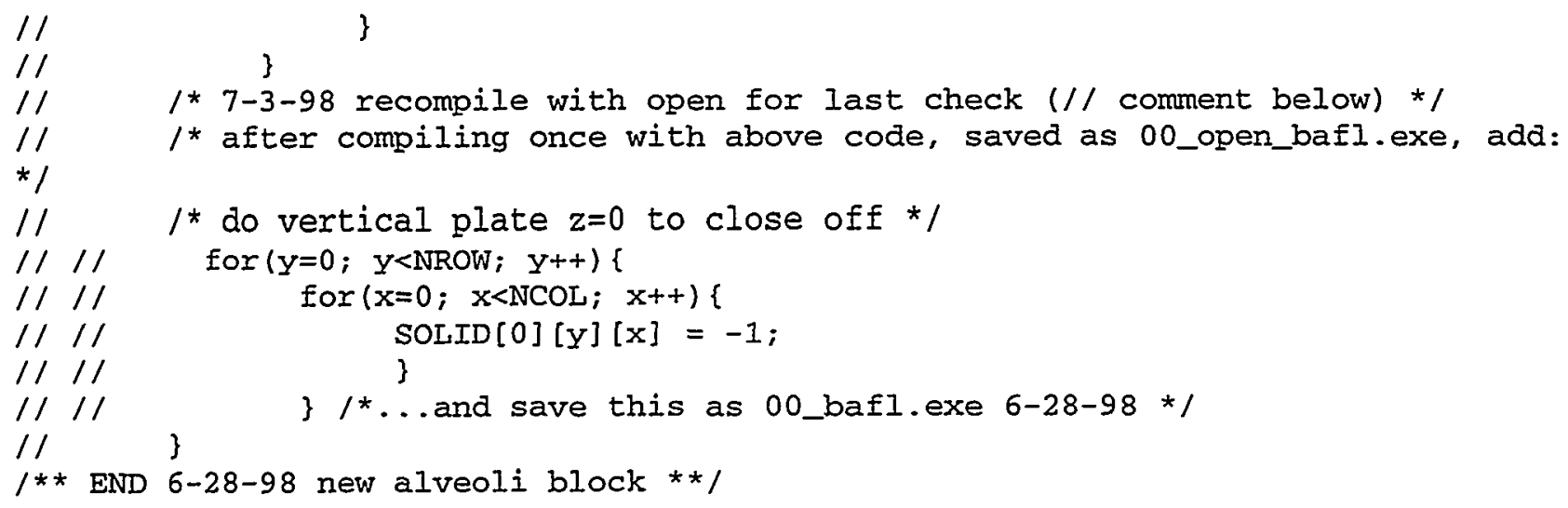


/* 7-9-97 add some inits of the UxAvg() params */

/* 6-14-98 change G.xUx to 0 (from NCOL-1) to capture between-solids Ux * /

$G \cdot x U x=0 ; \quad G \cdot y 1 U x=0 ; G \cdot y 2 U x=N R O W-1 ; G \cdot z 1 U x=0 ; G \cdot z 2 U x=N L A Y-1 ;$

G.YUY $=$ NROW-1; G.X1UY $=0 ; G \cdot x 2 U Y=N C O L-1 ; G \cdot z 1 U Y=0 ; G \cdot z 2 U x=$ NLAY $-1 ;$

G.zUz $=$ NLAY-1; G.Y1Uz $=0 ; G \cdot Y 2 U z=N R O W-1 ; G \cdot X 1 U z=0 ; G \cdot X 2 U z=N C O L-1 ;$

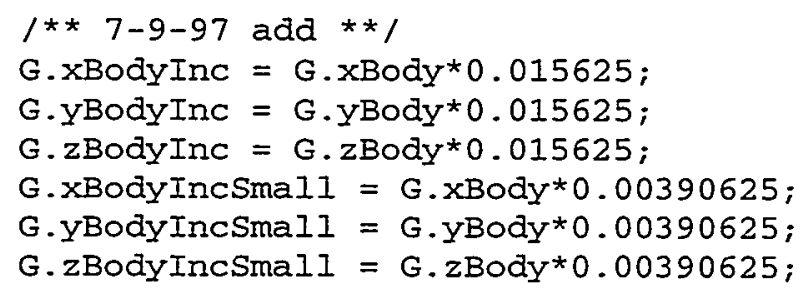




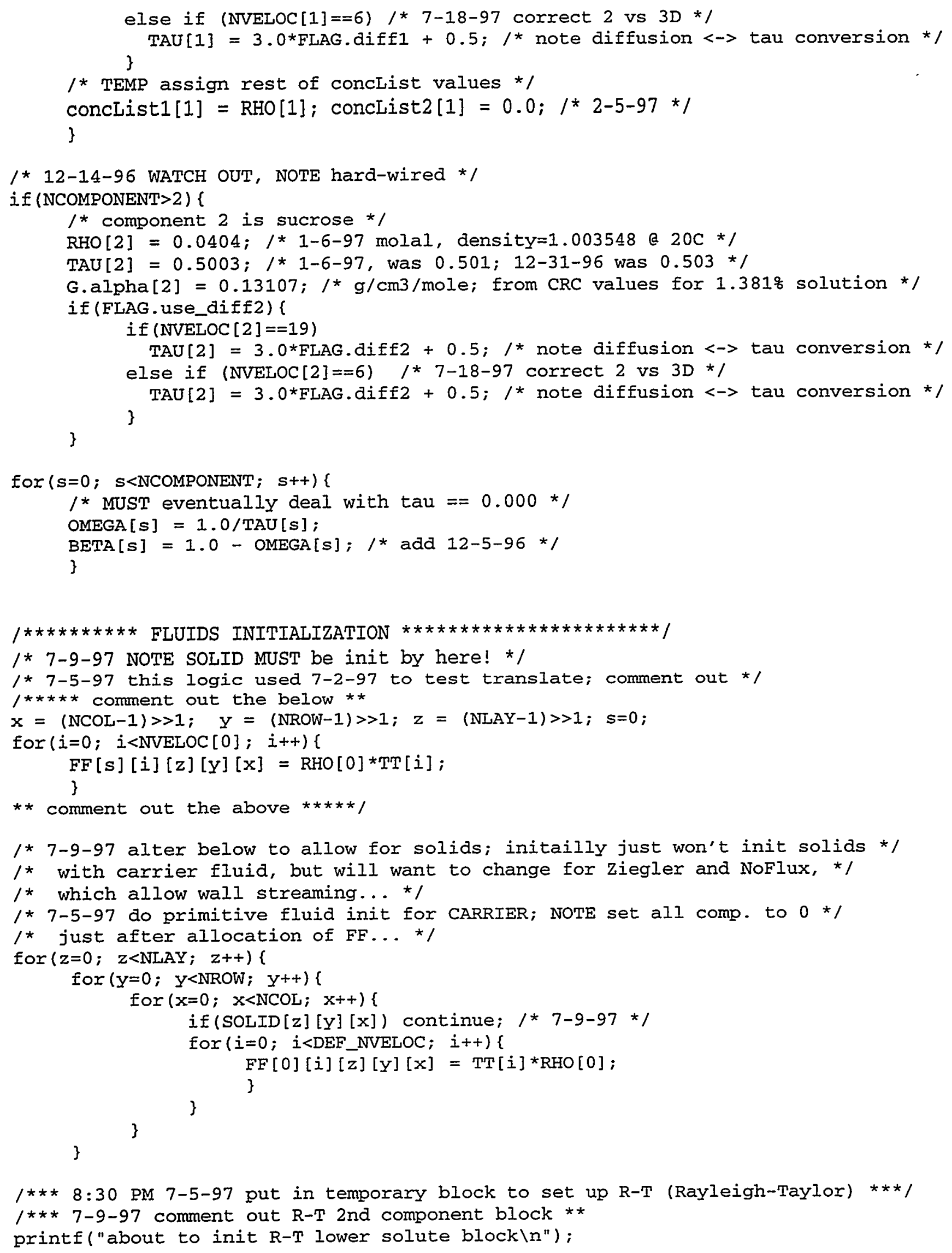




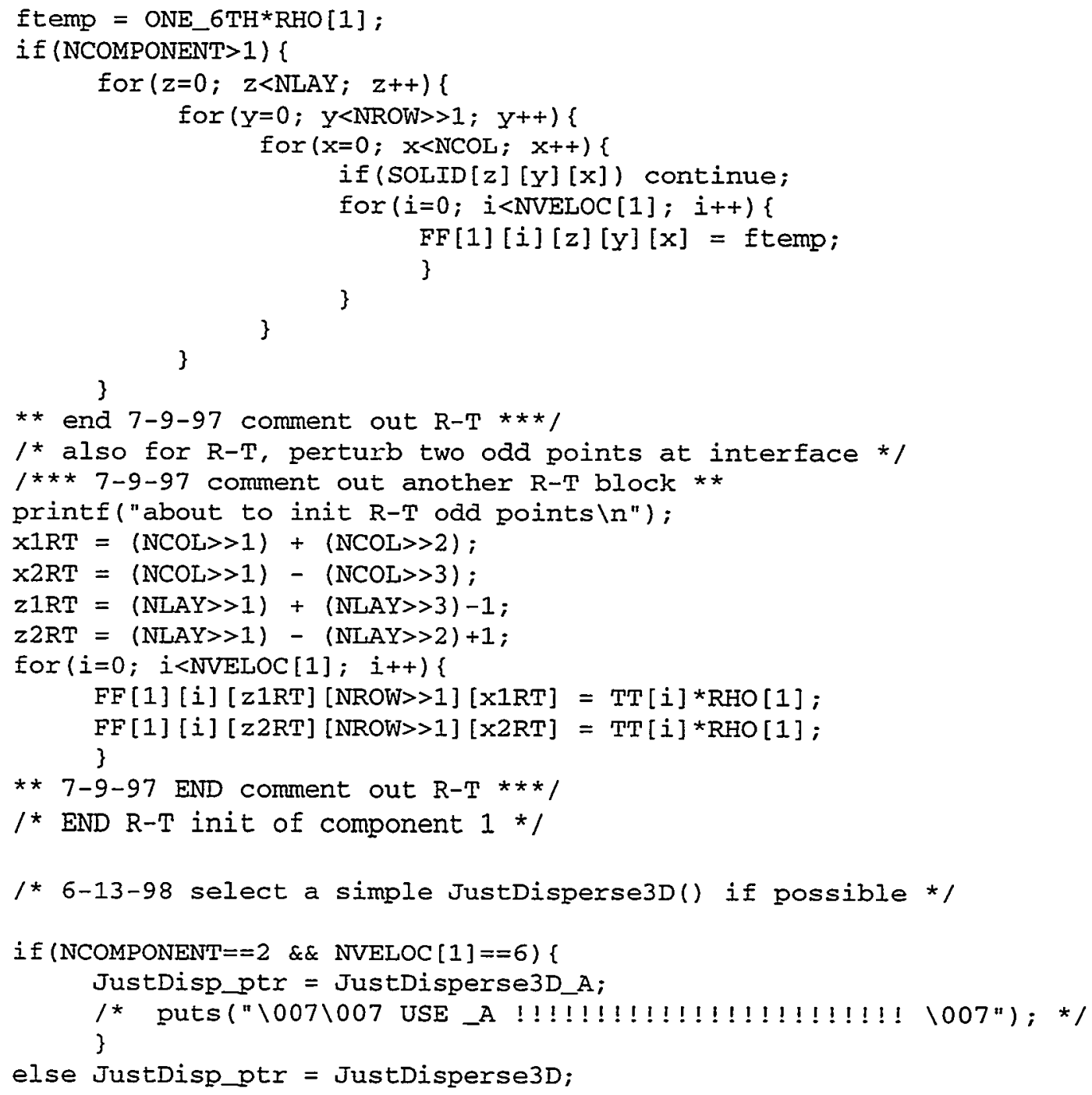




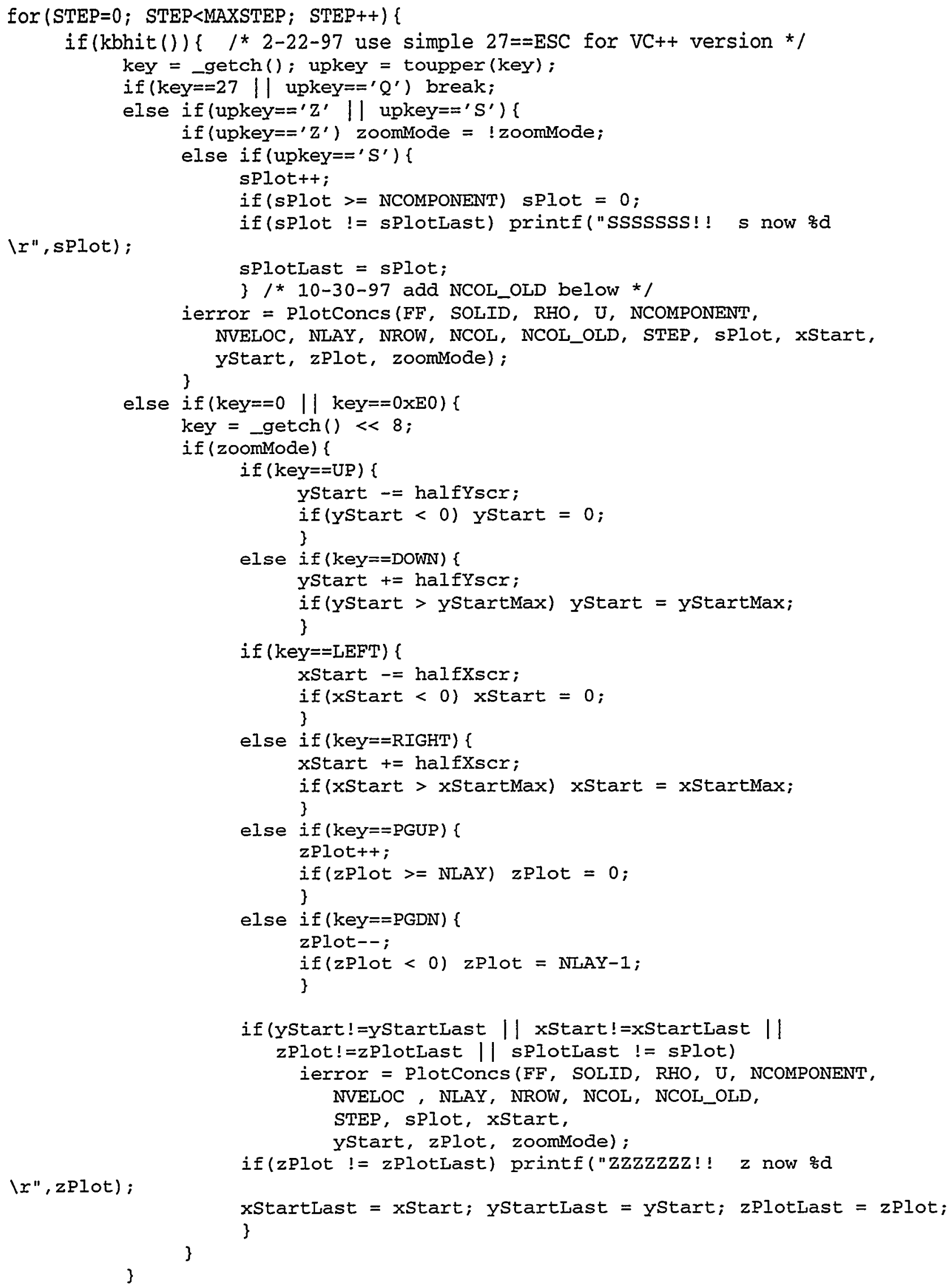


1 * Plot9Vec() call below typically used in isolation to test translate step; */

$1 *$ requires getch() to halt for inspection... */

$/ *$ ierror $=$ Plot9Vec (FF, RHO, NCOMPONENT, NVELOC, NLAY, NROW, NCOL, STEP); $/$

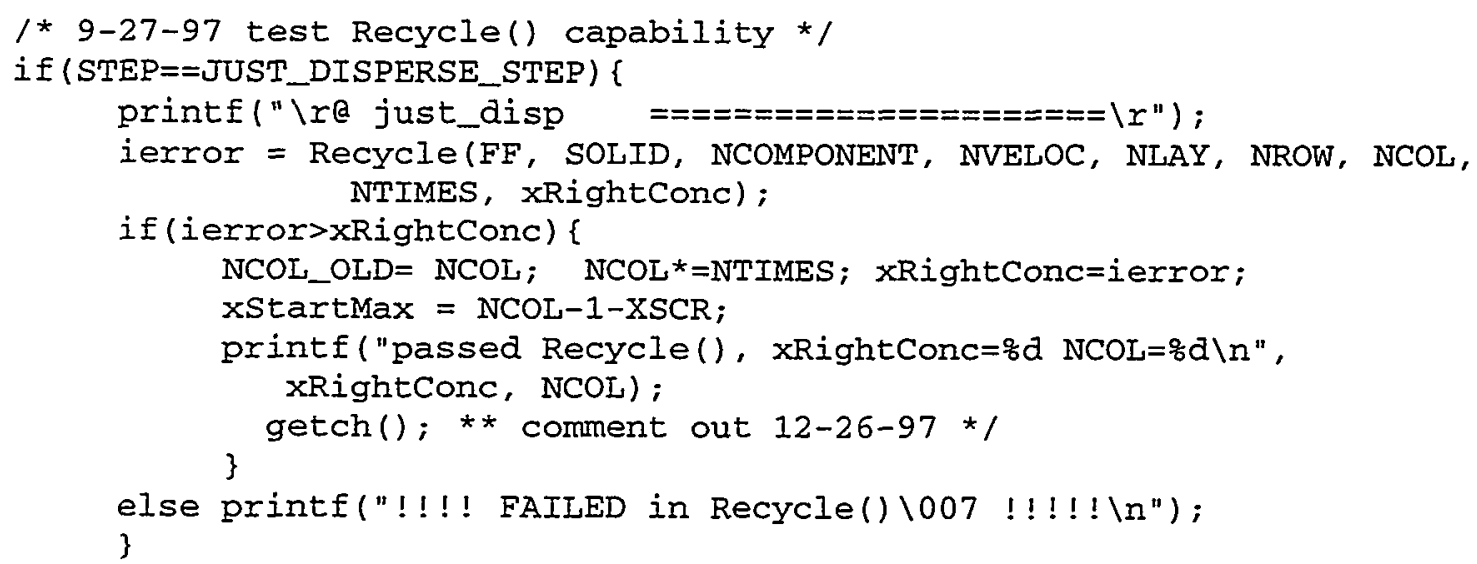




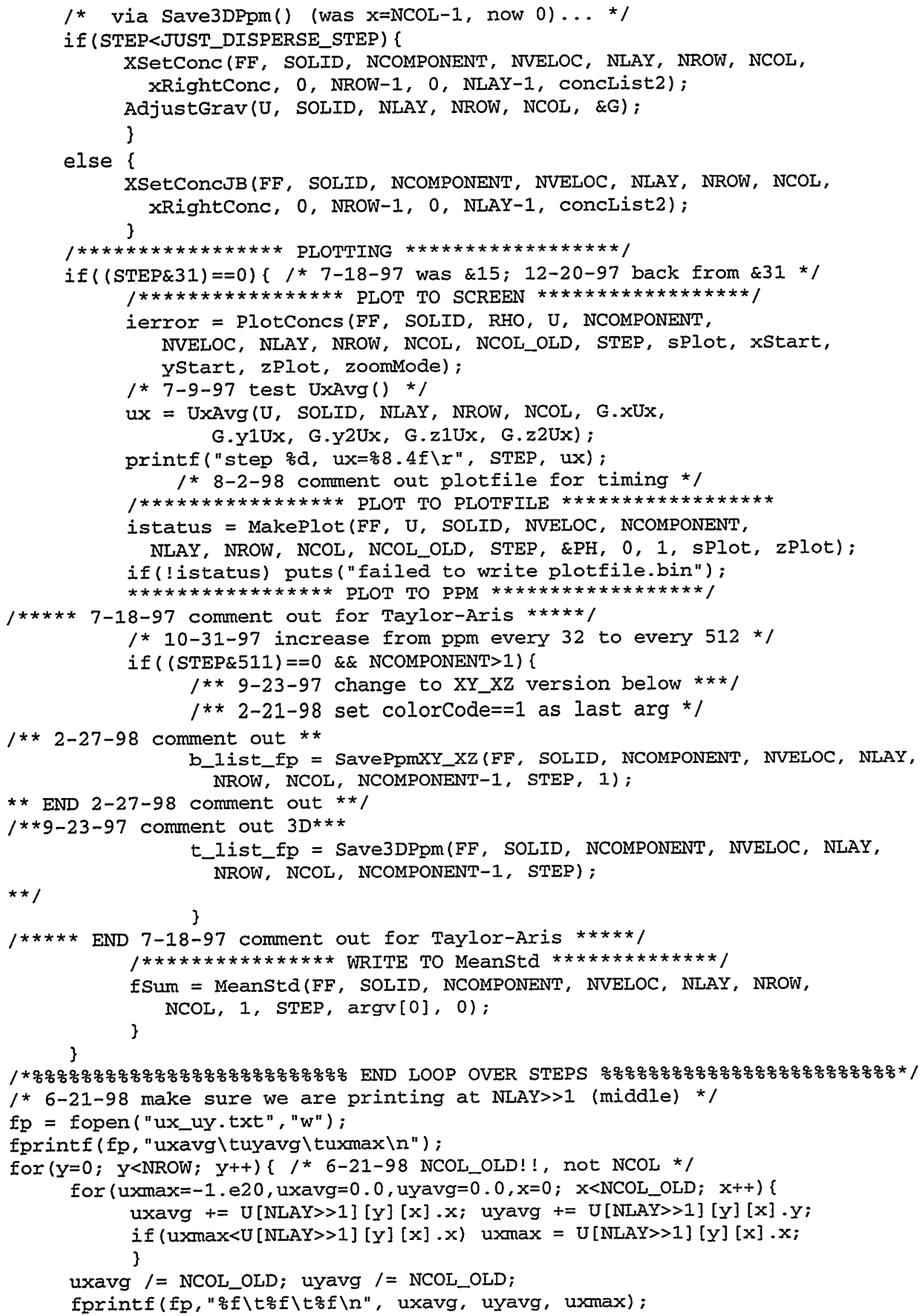




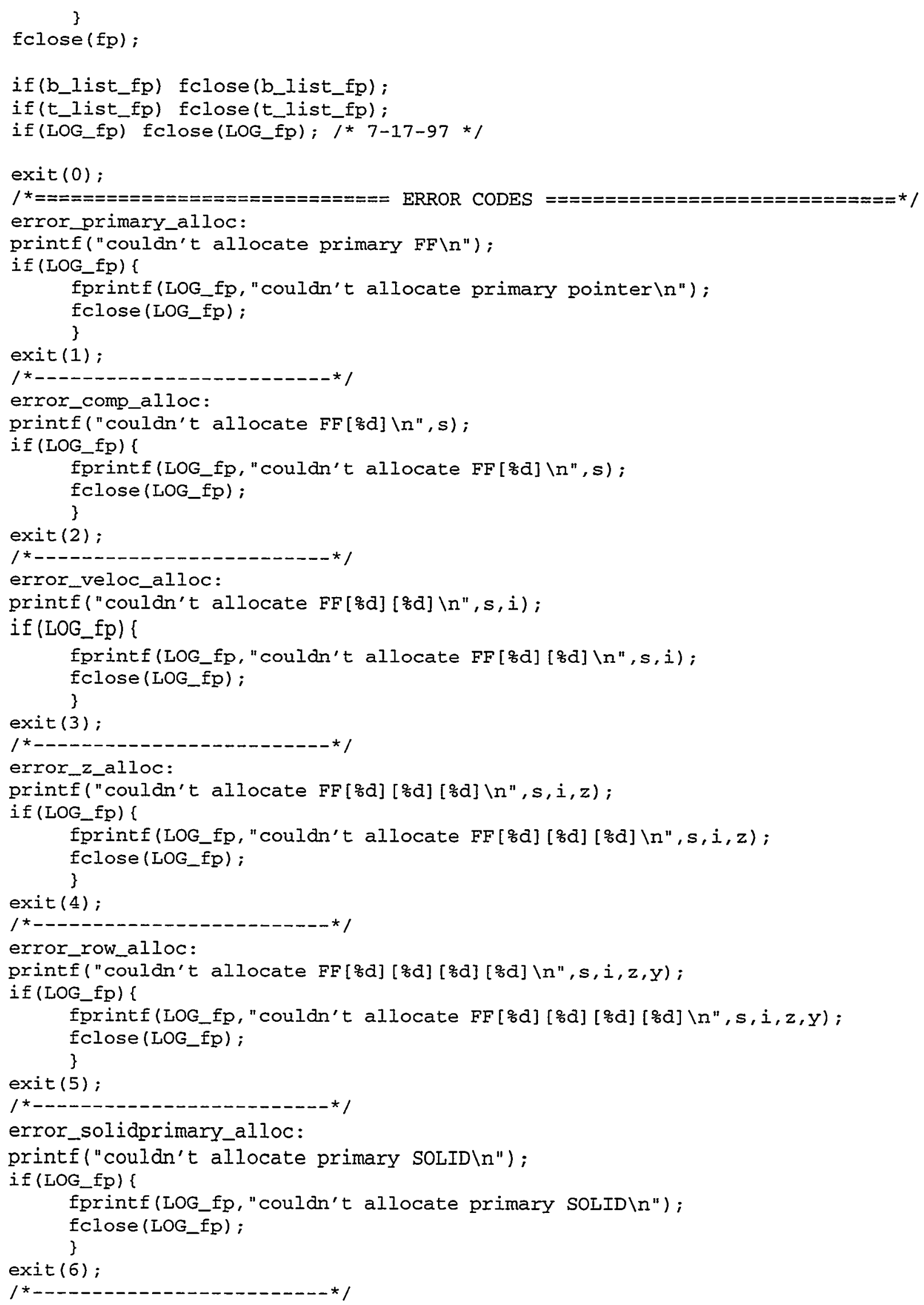




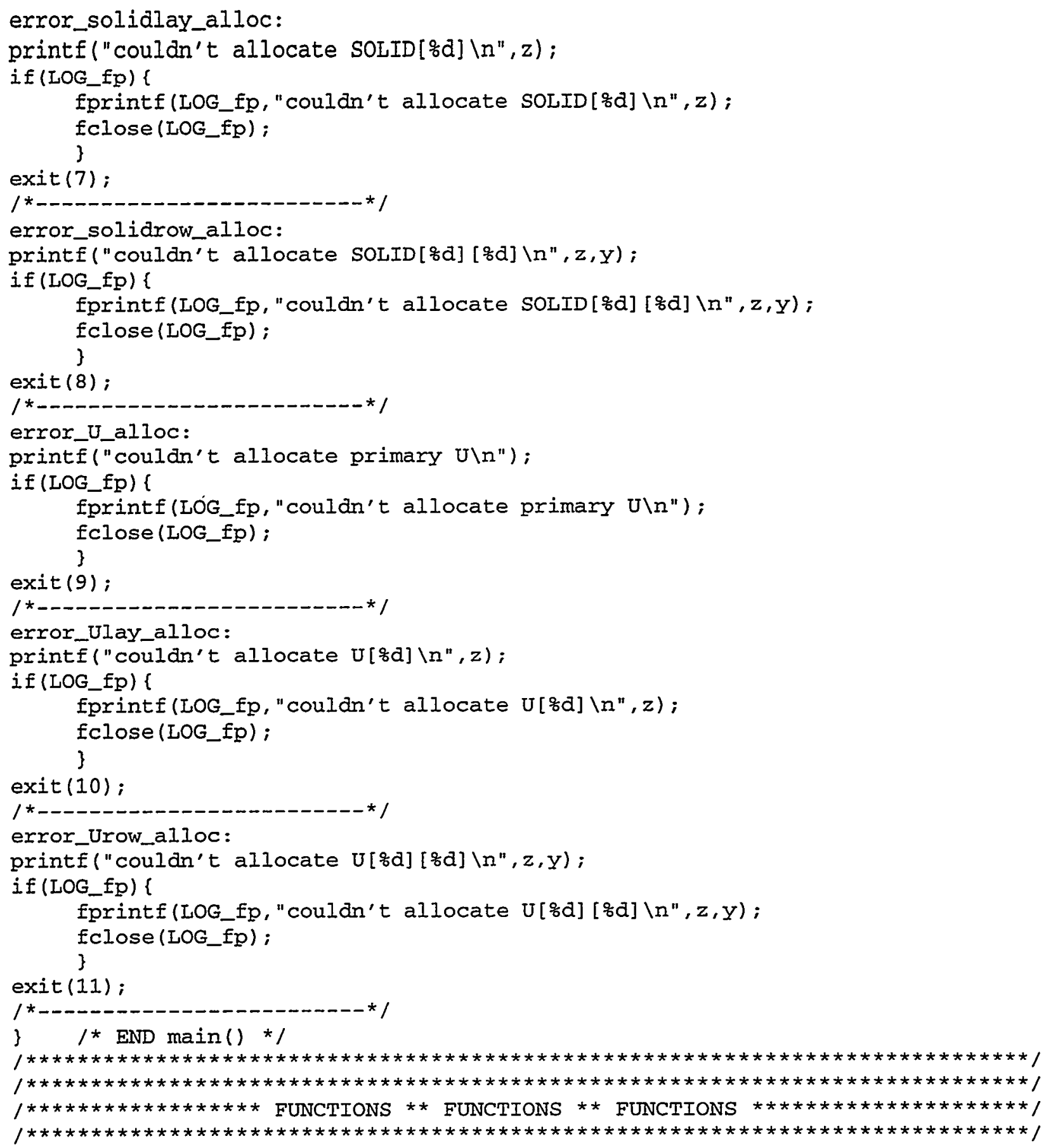




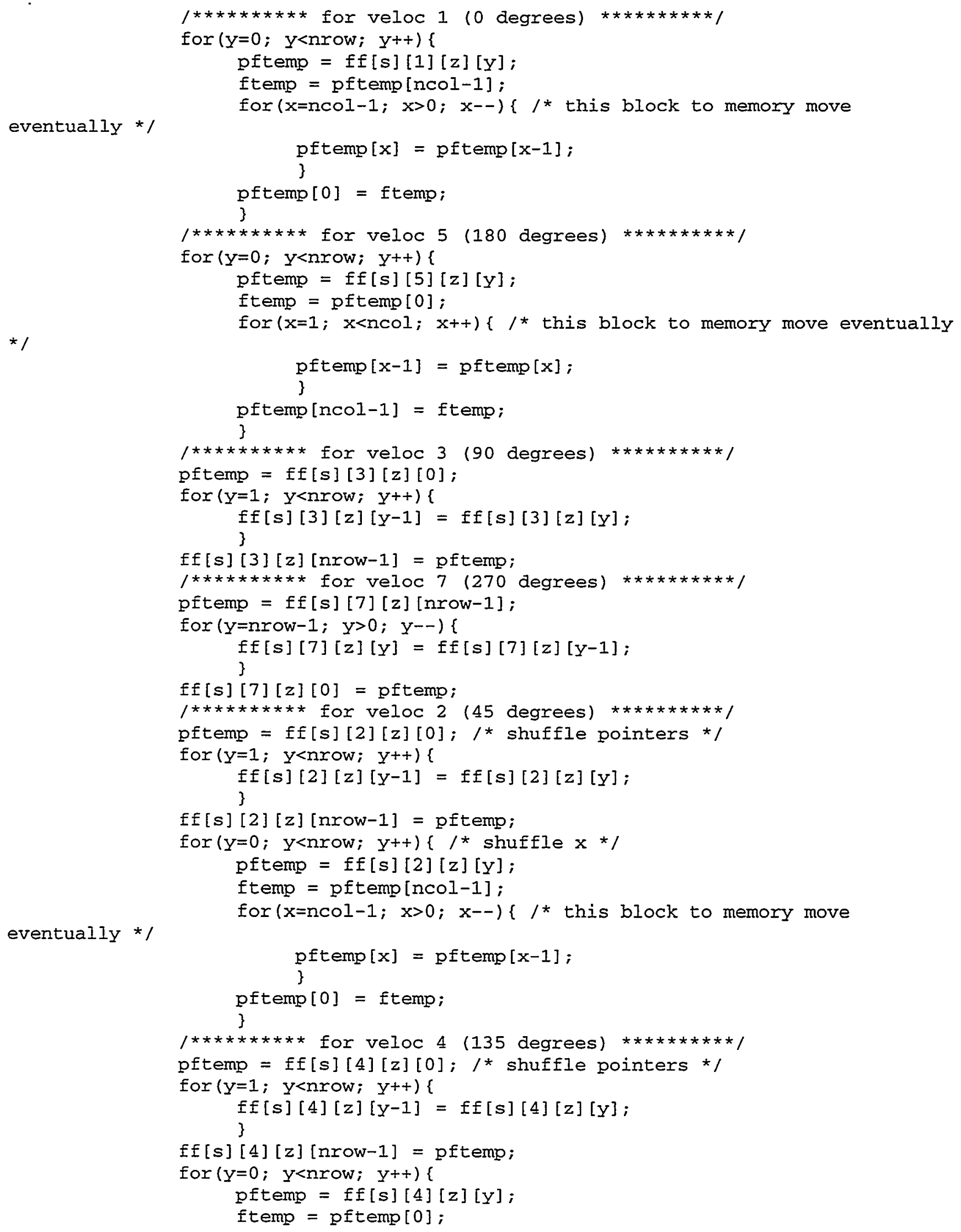




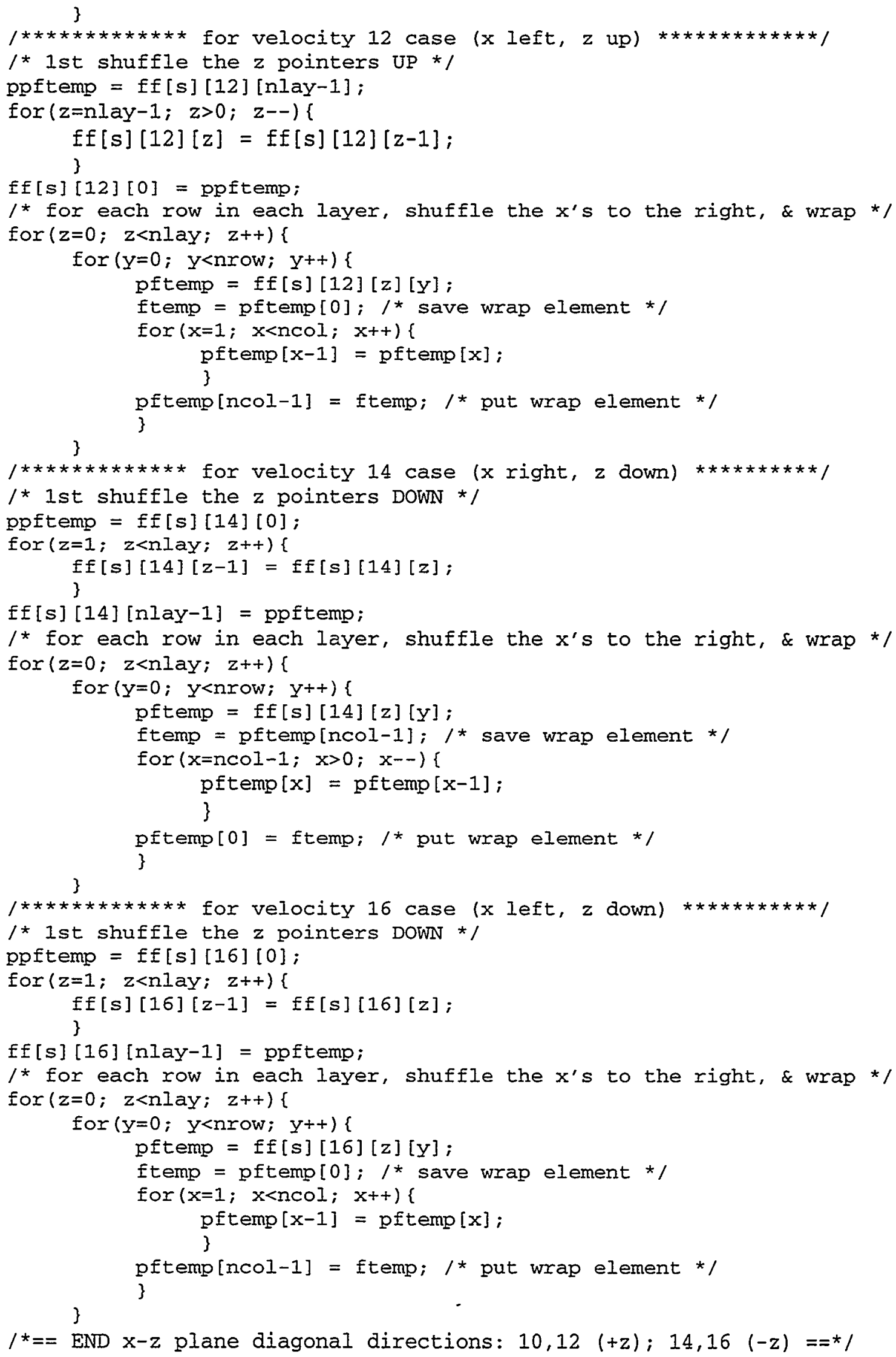




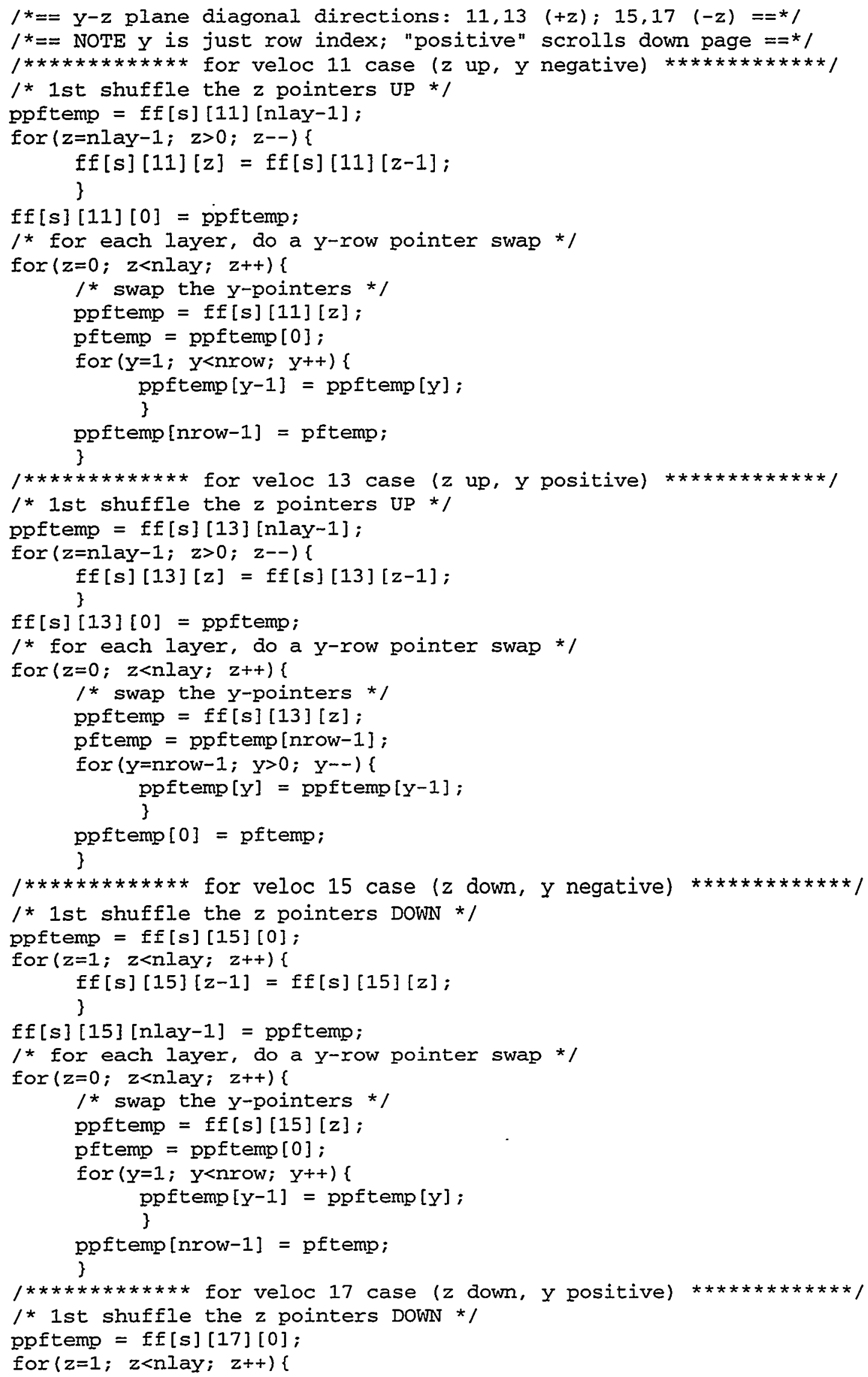




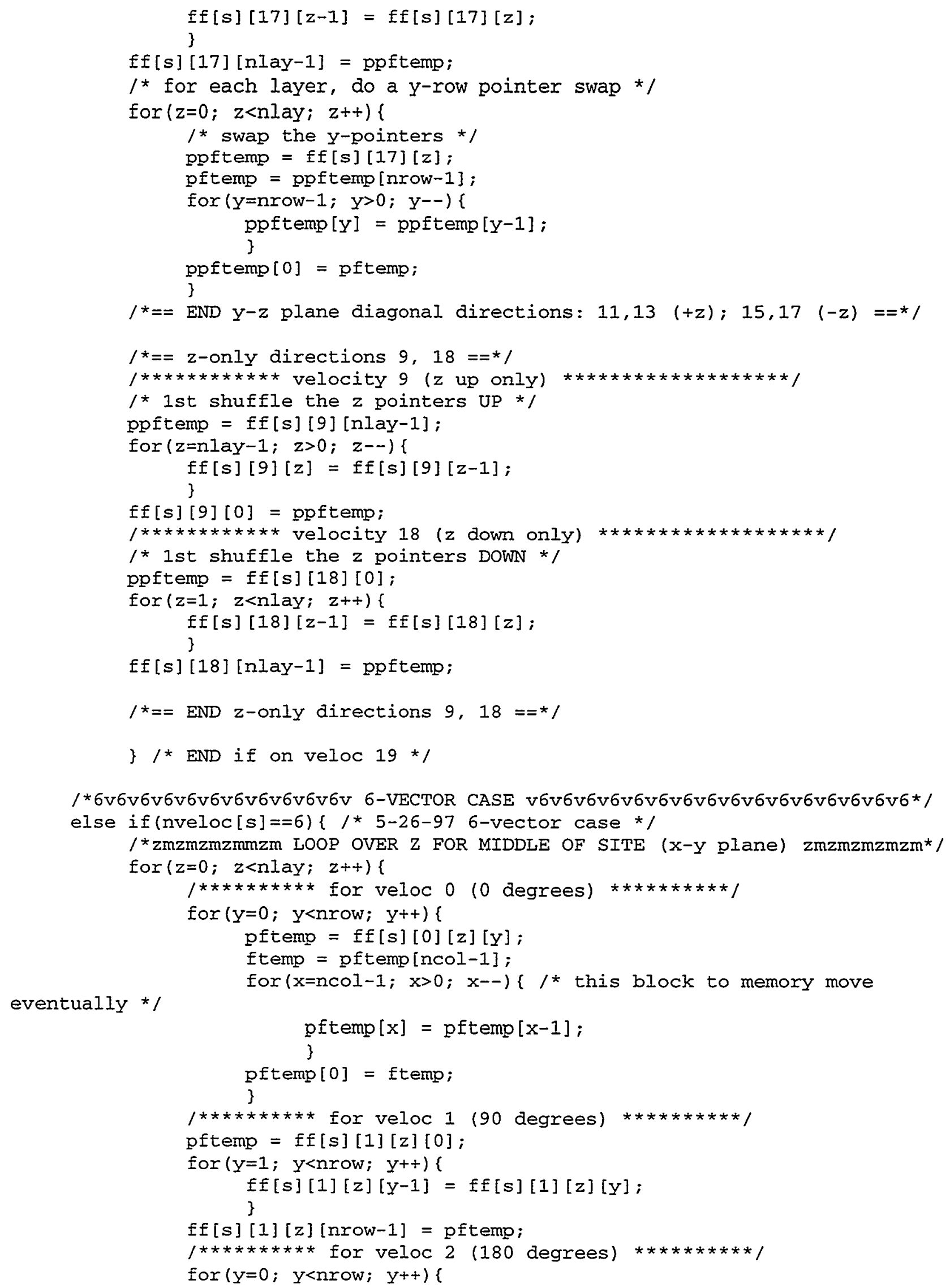




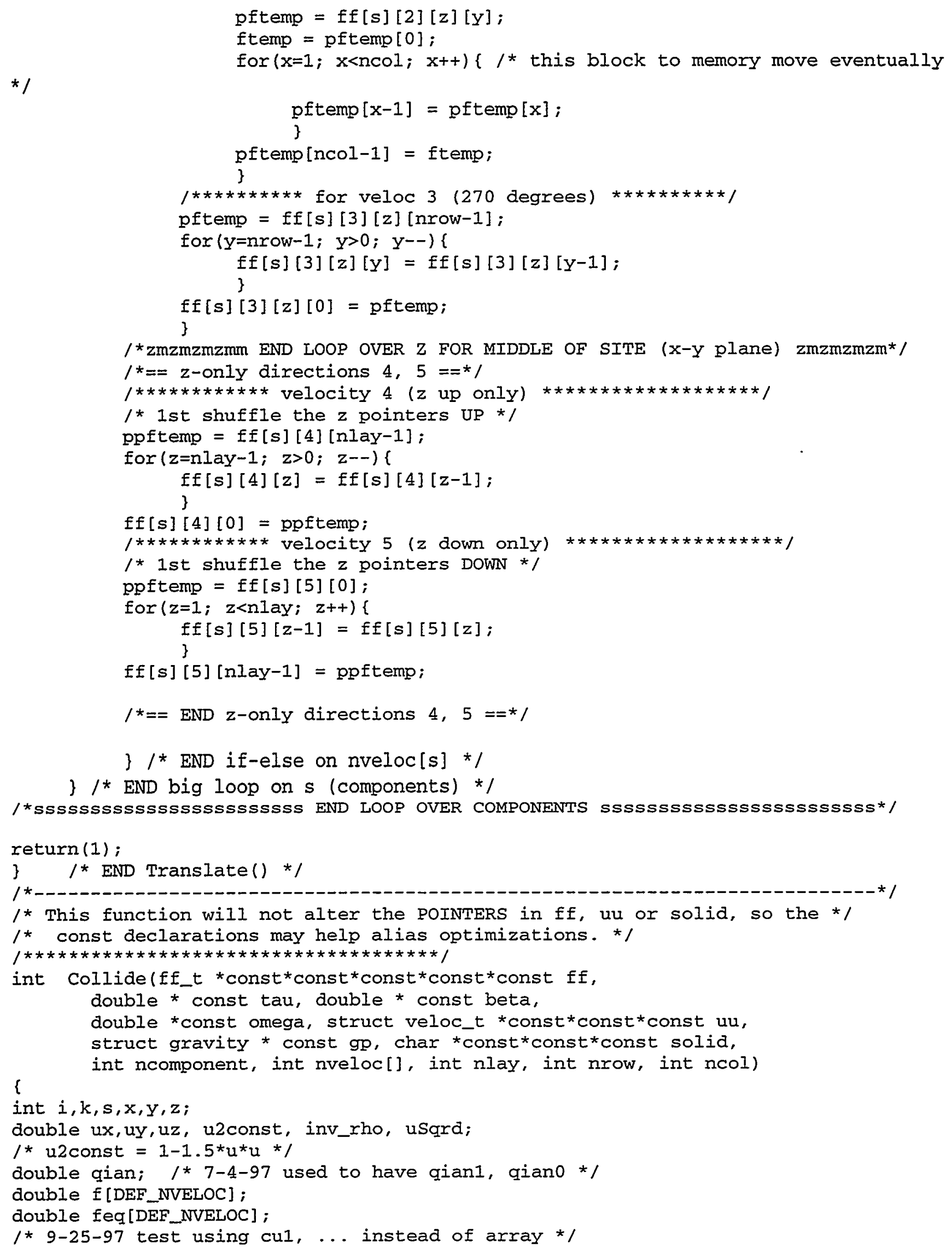


1 * double cu[DEF_NVELOC]; * 1

double cu1, cu2, cu3, cu4, cu5, cu6, cu7, cu8, cu9, cu10, cu11, cu12, cu13, cu14, cu15, cu16, cu17, cu18;

/*9-25-97*/

double mux, muy, muz;

$1 * 9-25-97 * 1$

ff_t ${ }^{*}$ const* const*const* ffs, *const*const*Const* ffo;

double tauo;

int solidcode;

double dux, duy, duz; $\quad /$ * gravity increments in ux, uy, uz */

double rho [MAX_COMP];

double del_density; $/ *$ true difference in physical density, between */

1 * reference solution and local solution at $x, y, z$ *

double omega_s, beta_s; $/ * 7-5-97 * /$

int doBounce, doziegler;

int nvel;

int do_buoy;

/* 7-5-97 allow us to skip buoyancy calc.; presume fabs() takes double */

do_buoy $=($ fabs (gp- $>x B u o y)<1 . e-30$ \&\& fabs (gp->yBuoy) $<1 . e-30$

$\& \&$ fabs (gp->zBuoy) $<1 . e-30)$ ? $0: 1$;

tauo $=\operatorname{tau}[0]$

ffo $=\mathrm{ff[0]:}$

for $(z=0 ; z<$ lay; $z++)\{$

for $(\mathrm{y}=0 ; \mathrm{y}<$ nrow $; \mathrm{y}++)$ \{

for $(\mathrm{x}=0 ; \mathrm{x}<\mathrm{ncol} ; \mathrm{x}++)\{$

solidcode $=$ solid $[z][y][x]$

/* 7-3-97 MUST classify solids before calling collide()! */

1 * solidcode -2 means completely surrounded by solids */

if ( solidcode $==-2$ ) continue;

1 * We calculate these only once */

doBounce $=$ (solidcode \&\& !USE_ZIEGLER) ||

(USE_ZIEGLER \&\& (solidcode<0 || solidcode>ZIEGLER_END_CODE));

doZiegler = USE_ZIEGLER \&\& solidcode>=ZIEGLER_IST_CODE

\&\& Solidcode<=ZIEGLER_END_CODE;

1 * first do the carrier fluid (first index $s=0$ ) */

/* make local copies of all component 0 (fluid) vectors */

rho $[0]=f[0]=f f 0[0][z][y][x]$

rho[0] $+=f[1]=f f o[1][z][y][x]$;

rho[0] $+=f[2]=f f 0[2][z][y][x]$;

$r h o[0]+=f[3]=f f o[3][z][y][x] ;$

rho[0] $+=f[4]=$ ffo[4][z][y] $[x]$

rho $[0]+=f[5]=f f 0[5][z][y][x] ;$

rho[0] $+=f[6]=f f o[6][z][y][x] ;$

rho[0] $+=f[7]=f f 0[7][z][y][x]$;

rho[0] $t=f[8]=f f 0[8][z][y][x]$

rho[0] $+=f[9]=$ ffo[g][z][y] $[x]$

rho[0] $t=f[10]=f f 0[10][z][y][x]$;

rho[0] $t=f[11]=f f 0[11][z][y][x] ;$

rho[0] $t=f[12]=$ ffo[12][z][Y] $[\mathrm{x}]$;

rho[0] $t=f[13]=f f 0[13][z][y][x] ;$

rho[0] $t=f[14]=f f o[14][z][y][x] ;$

rho[0] $t=\mathrm{f}[15]=$ ffo[15][z][y][x]; 
rho[0]+=f[16] $=$ ffo[l16][z][y][x];
rho[0]+=f[17] = ffo[17][z][y][x];
rho[0]+=f[18] $=$ ffo[18][z][y][x];

if (doziegler) rho[0] = Ziegler (E, solidcode, 1 );

if $(r h o[0]<1.0 e-30)$ continue; $/$ * denotes a problem */

/* get rho other components-- */

/* 7-3-97 NOTE we should have functions without buoyancy, and with only */

/* 2 or 3 components; without buoy, we would eliminate the double read */

$1^{*}$ of $\mathrm{ff}[\mathrm{s}]$ (which may pre-cache, so could be OK)...*/

for $(s=1 ; s<$ ncomponent; $s++)\{$

ffs $=f f[s] ;$

rho[s] = ffs $[0][z][y][x]$

rho[s] $t=$ ffs $[1][z][y][x]$;

rho[s] $+=$ ffs [2][z] $[y][x]$;

rho[s] $+=$ ffs[3][z] $[y][x]$;

rho[s] $+=$ ffs [4][z][y][x];

rho[s] $+=$ ffs [5] [z] [y] [x];

if (nveloc $[s]>6)\{/ *$ kludgey, 5-26-97 */

rho $[s]+=$ ffs $[6][z][y][x]$;

rho[s] $+=$ ffs $[7][z][y][x]$;

rho[s] $+=$ ffs $[8][z][y][x]$;

rho[s] $+=$ ffs $[9][z][y][x]$;

rho[s] $+=$ ffs $[10][z][y][x]$;

rho[s] $+=$ ffs $[11][z][y][x]$;

rho[s] $+=$ ffs [12][z] $[y][x]$;

rho[s] $+=$ ffs [13][z] [y] [x];

rho[s] $+=$ ffs [14][z] $[y][x]$;

rho[s] $+=$ ffs $[15][z][y][x]$;

rho $[s]+=$ ffs $[16][z][y][x]$;

rho[s] $+=$ ffs [17] [z] $[y][x]$;

rho[s] $+=$ ffs $[18][z][y][x]$;

\}

\}

/* simple bounceback block */

if (doBounce) \{

$\mathrm{uu}[z][y][\mathrm{x}] \cdot \mathrm{x}=0.0 ; \quad \mathrm{uu}[z][y][\mathrm{x}] \cdot \mathrm{y}=0.0 ;$

uu $[z][y][x] . z=0.0 ;$ uu $[z][y][x]$.usqrd $=0.0 ;$

BounceBack $(f)$;

for $(i=1 ; i<$ nveloc $[0] ; i++)$ ffo[i] $[z][y][x]=f[i] ;$

\}

else

inv_rho $=1.0 /$ rho $[0]$;

$u x=$ inv_rho* $(f[1]+f[2]+f[8]+f[10]+f[14]$

$-f[5]-f[4]-f[6]-f[12]-f[16]) ;$

$u y=i n v \_r h o *(f[2]+f[3]+f[4]+f[11]+f[15]$

$-f[6]-f[7]-f[8]-f[13]-f[17]) ;$

$u z=$ inv_rho* $(f[9]+f[10]+f[11]+f[12]+f[13]$

- $f[14]-f[15]-f[16]-f[17]-f[18])$;

* 7-4-97 SHOULD put in logic to skip buoyancy if not needed */

$1 * * * * * *$ forcing, buoyancy block $* * * * \star * /$

dux $=$ gp->txBody; duy = gp->tyBody; duz = gp->tzBody;

if (do_buoy) \{

for (del_density $=0.0, k=1 ; k<$ component; $k++$ ) \{ del_density $t=r h o[k] *(g p->a l p h a[k])$; \}

$/ * *$ 1-17-97 kludgey, use a global here **/ 
if (rho[0]>1e-10) del_density $*=$ RHO [0]/rho[0]; del_density $-=$ DEL_DENSITY_REF;

dux $+=$ del_density* (gp->xBuoy);

duy $+=$ del_density* (gp $>$ yBuoy);

duz $+=$ del_density* (gp->zBuoy); \}

ux $+=$ dux*tau0; uy $+=$ duy*tau0; uz $+=$ duz*tau0;

1 * store for later *I

usqrd $=u x^{\star} u x+u y^{\star} u y+u z^{\star} u z$;

uu $[z][y][x] \cdot x=u x ; \quad u u[z][y][x] \cdot y=u y ;$

$u u[z][y][x] . z=u z ; \quad u u[z][y][x]$.usgrd = usqrd;

u2 const $=1.0-1.5 *$ usgrd;

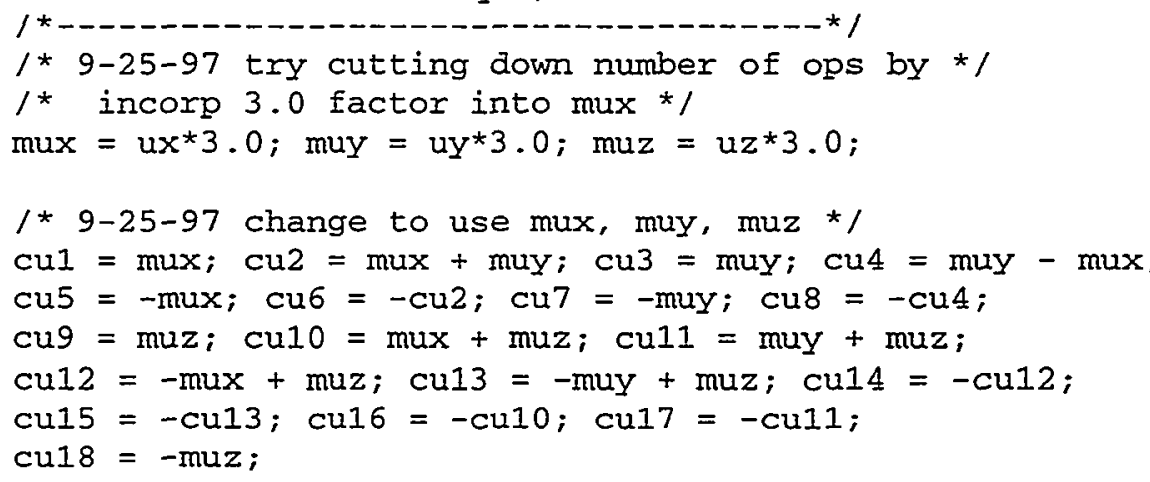

$I^{*}$ when we have debugged, factor below with simpler */

1 * expressions or consider eliminating cu array, and *

1 * calc each cu[] in place ... * $/$

feq $[0]=$ ONE_3RD*rho $[0]{ }^{*} \mathrm{u} 2$ const;

qian $=r h o[0] * O N E \_18 T H$;

$f * 7-4-97$ old had giano $=$ rho $[0] * \mathrm{ONE} \_36 \mathrm{TH} ; * /$

/* the Cartesian directions */

feq $[1]=$ qian* $\left(u 2\right.$ const + cul $\left.+0.5^{\star} \mathrm{cu} 1{ }^{\star} \mathrm{cu} 1\right)$;

feq $[3]=$ qian $*\left(u 2\right.$ const + cu $\left.3+0.5^{*} \operatorname{cu} 3 * \operatorname{cu} 3\right) ;$

feq $[5]=$ qian* (u2const + cu $5+0.5 *$ cu $5 *$ cu 5$)$;

feq $[7]=$ qian $*(u 2$ const $+\operatorname{cu} 7+0.5 * \operatorname{cu} 7 * \operatorname{cu} 7) ;$

feq $[9]=$ qian* $\left(u 2\right.$ const + cug $+0.5^{*}$ cug*cug);

feq $[18]=$ qian* $($ u2const + cul $18+0.5 * \operatorname{cu} 18 *$ cu18);

$I *$ the off-axis, diagonal directions * $/$

qian ${ }^{*}=0.5 ; I^{*}$ may have to go back to qian 1 , qiano */

feq $[2]=$ qian* $(u 2$ const $+\mathrm{cu} 2+0.5 * \operatorname{cu} 2 * \mathrm{cu} 2) ;$

feq $[4]=$ gian* (u2const + cu $4+0.5 *$ cu $4 *$ cu 4$)$;

feq $[6]=$ qian* $(u 2$ const + cu $6+0.5 *$ cu $6 *$ cu 6$)$;

feq $[8]=$ qian* $(u 2$ const + cu $8+0.5 *$ cu $8 *$ cu 8$)$;

feq $[10]=$ qian $*\left(u 2\right.$ const + cu10 $+0.5 *$ cul $1{ }^{*}$ cu10 $) ;$

feq $[11]=$ qian* $(u 2$ const $+\operatorname{cul1}+0.5 * \operatorname{cul1} 1 *$ cul1 $)$;

feq $[12]=$ gian $*(u 2$ const + cul2 $+0.5 * \operatorname{cul} 1 * \operatorname{cu} 12)$;

feq $[13]=$ gian $*\left(u 2\right.$ const $\left.+\operatorname{cul} 13+0.5 * \operatorname{cul} 1{ }^{*} \operatorname{cu} 13\right)$;

feq $[14]=$ qian* $\left(u 2\right.$ const $\left.+\operatorname{cul} 4+0.5^{*} \operatorname{cu} 14 * \operatorname{cu} 14\right) ;$

feq $[15]=$ qian* $(u 2$ const $+\operatorname{cu} 15+0.5 * \operatorname{cu} 15 * \operatorname{cu} 15) ;$

feg $[16]=$ qian* $(u 2$ const $+\operatorname{cu} 16+0.5 * \operatorname{cu} 16 *$ cul 6$) ;$

feq $[17]=$ qian* $(u 2$ const $+\operatorname{cul} 1+0.5 * \operatorname{cu} 17 * \operatorname{cu} 17)$;

\section{if (doziegler) \{}

Ziegler ( $f$, solidcode, 0$)$;

Ziegler(feq, solidcode, 0); 


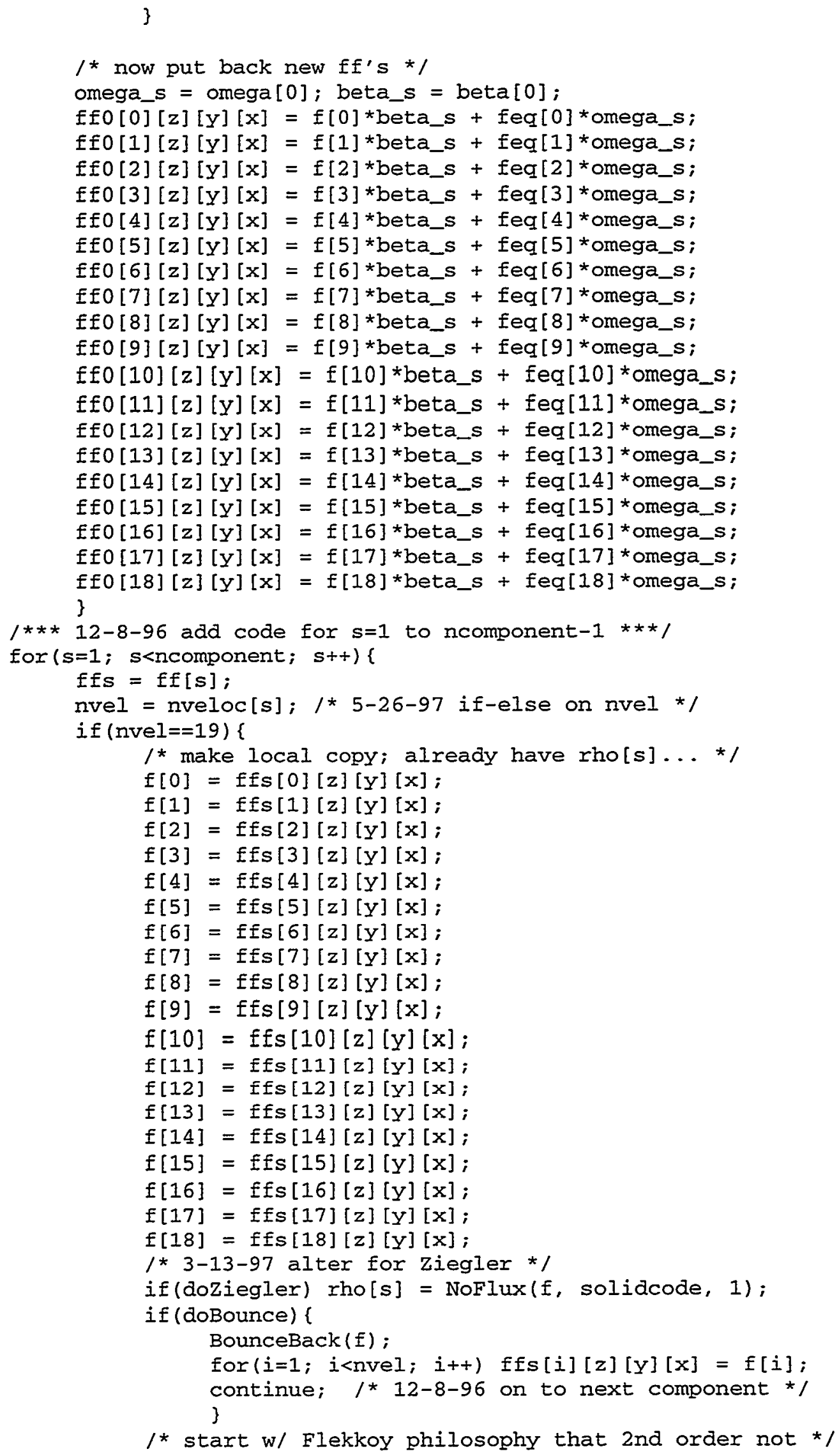




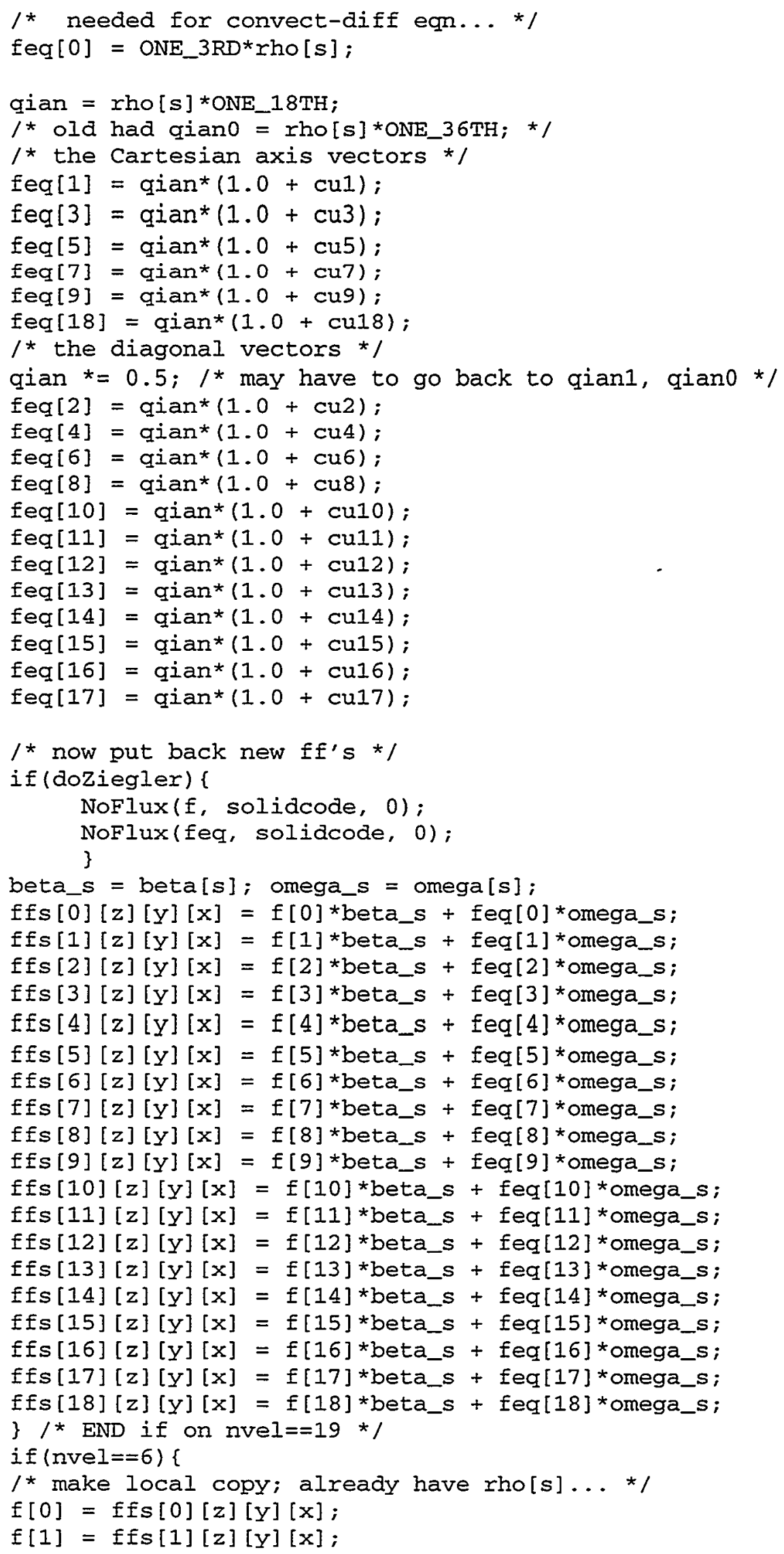




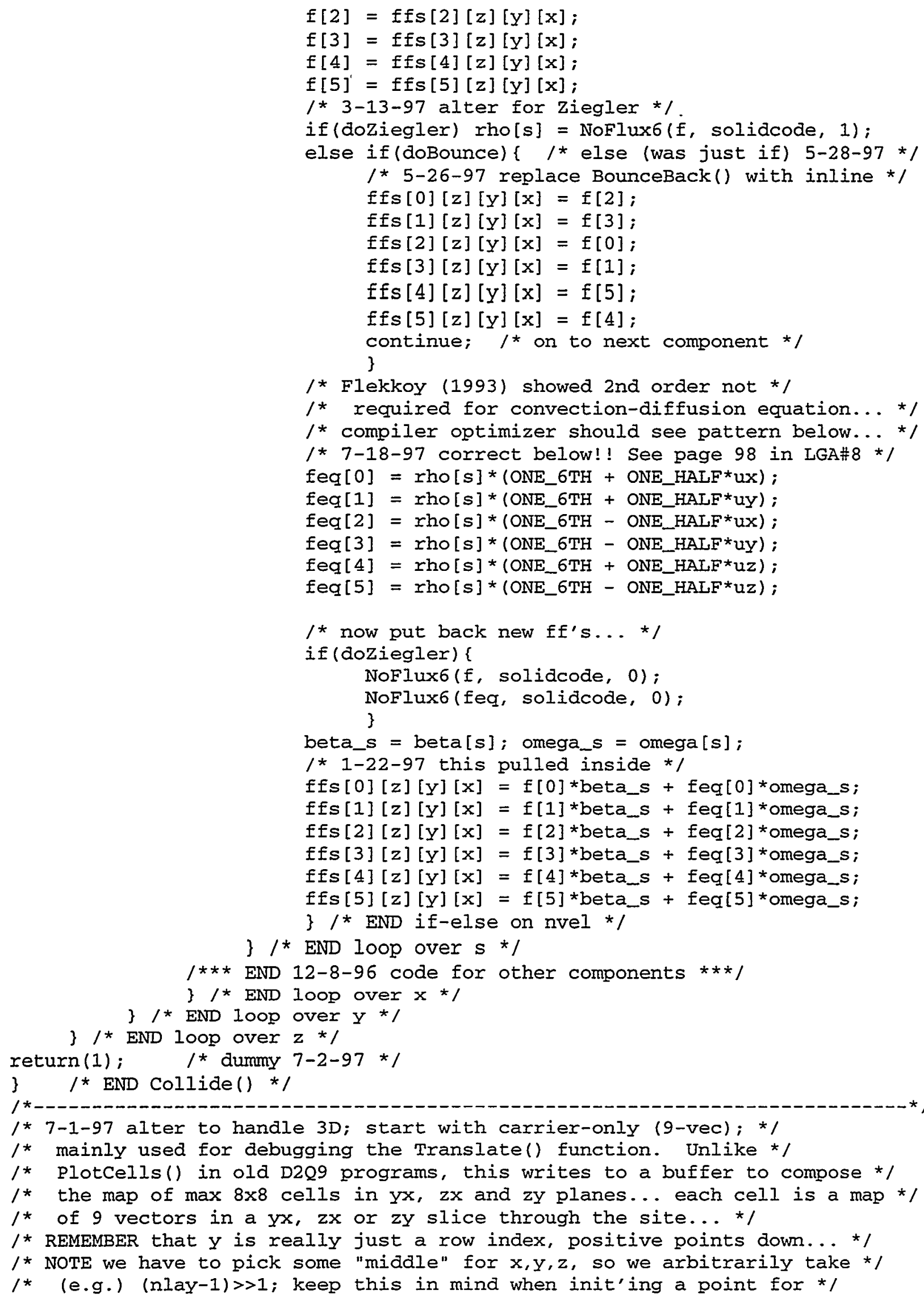




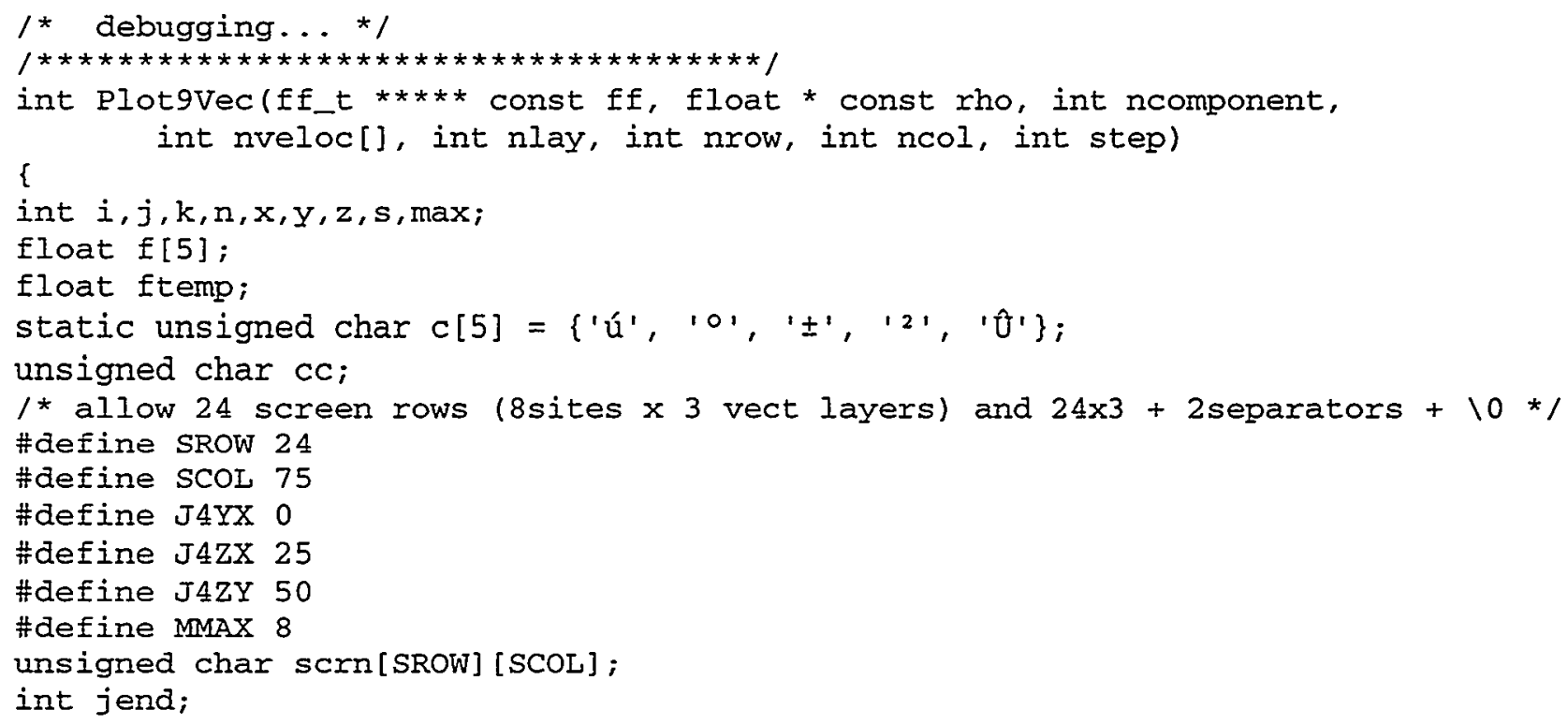




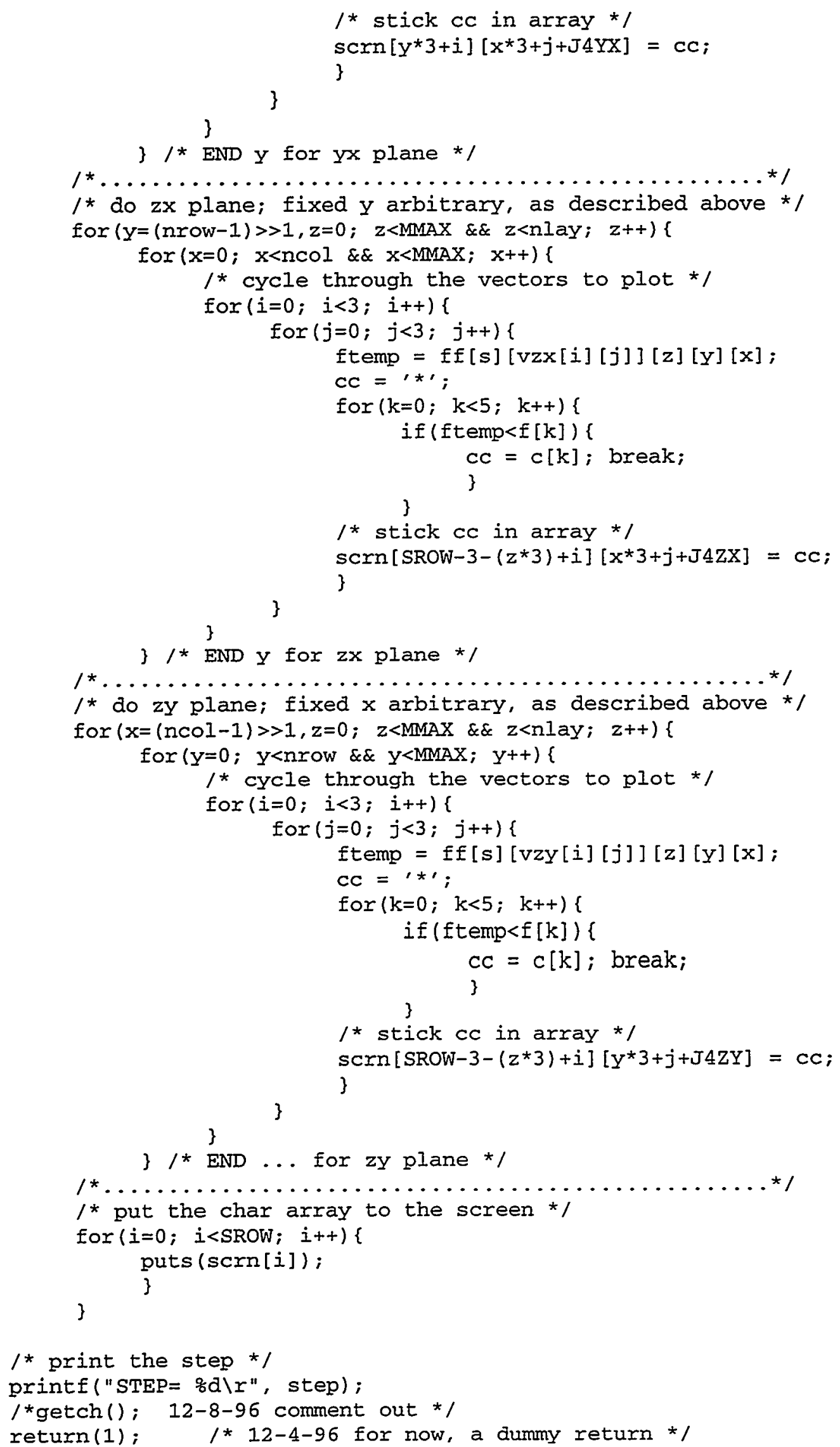




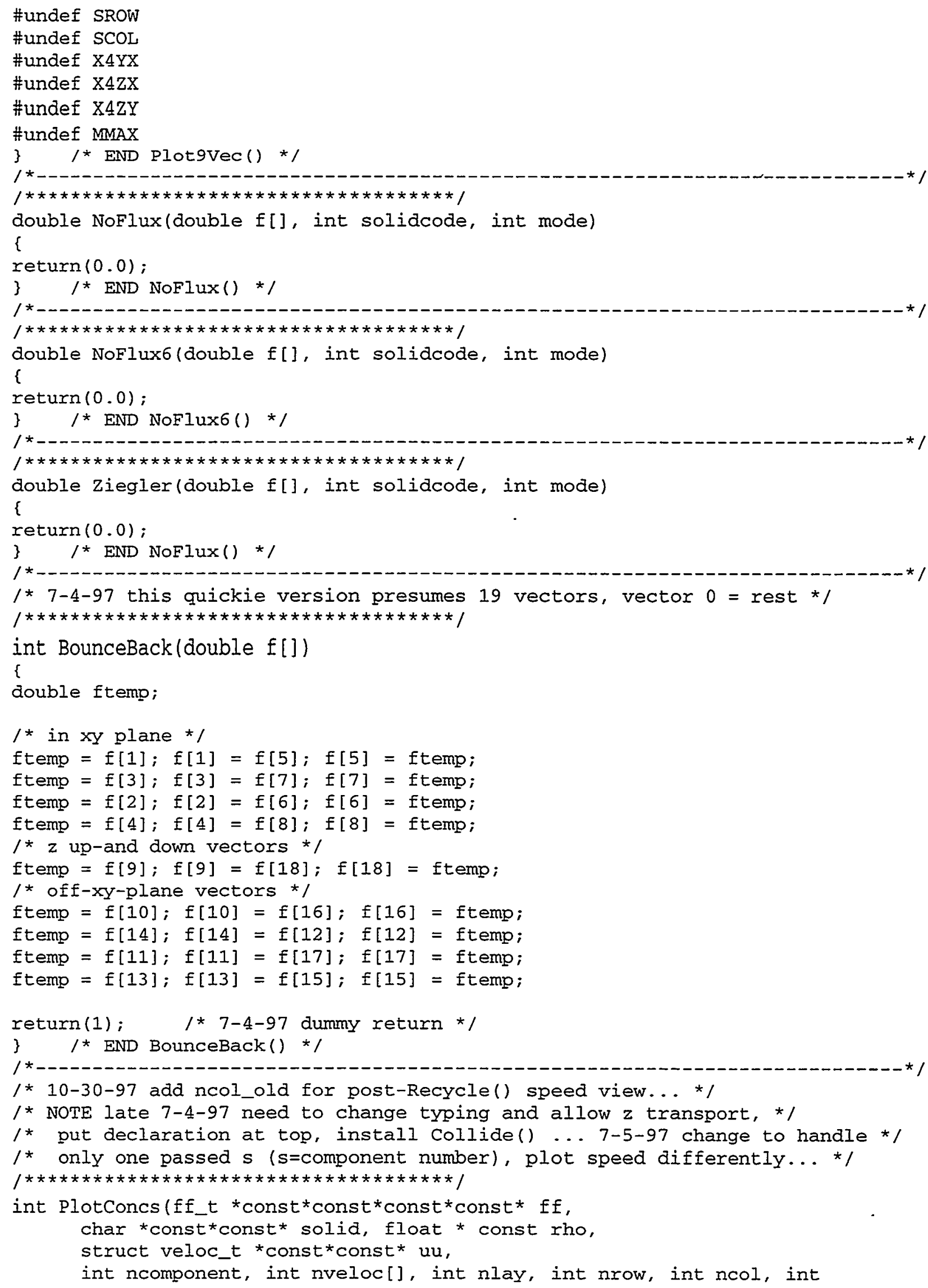


ncol_old, int step, int $s$, int xStart, int ystart, int $z z$, int zoomMode)

\{

int $i, k, n, x, y, z, x P e r P i x, y P e r P i x, x 0, y 0, x x, y y$;

float $f[5]$;

ff_t $t * * * * f f_{\text {; }}$

float ftemp, umax;

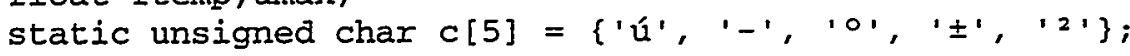

unsigned char cc;

int $\mathrm{xxx} ; \quad$ * 10-30-97 for modding after Recycle() */

if $(z z<0|| z z>=n l a y)$ return(0);

if (! zoomMode) (

xPerPix $=($ XSCR<NCOL $) ?($ NCOL $+($ XSCR $>1)) /$ XSCR : 1 ;

yPerPix $=($ YSCR<NROW $) ?($ NROW $+($ YSCR $>1)) /$ YSCR : 1;

$\mathrm{x} 0=0 ; \mathrm{y}^{0}=0 ;$

else \{

$x \operatorname{PerPix}=$ yPerPix $=1$;

$\mathrm{x} 0=\mathrm{xStart} ; \mathrm{y} 0=\mathrm{yStart} ;$

\}

$\mathrm{ffs}=\mathrm{ff}[\mathrm{s}]$

system("cls"); /* Unix: system("clear"); Unix || Mac: printf("\f"); */

if $(s>0)\{1$ * treat solutes *

/* Set up levels of gradation (if-else) */

if (nveloc [s] $==19$ )

$f[4]=r h o[s] * O N E \_3 R D ;$

$E[2]=x h o[s] * \mathrm{ONE} \_6 \mathrm{TH} ;$

$f[0]=r h o[s] * \mathrm{ONE} \_36 \mathrm{TH}$;

else \{

\}

$f[4]=r h o[s] ; f[2]=r h o[s] * O N E \_6 T H ; f[0]=r h o[s] * 0.05 ;$

$f[1]=(f[0]+f[2]) * 0.5$;

$f[3]=(f[2]+f[4]) * 0.5 ;$

for $(n=0 ; n<5 ; n++) f[n] *=0.9$;

for $(y=0, y Y=y 0 ; y<n$ row\&\&y<YSCR; $y++, y Y+=y$ Per $P i x)\{$

for $(x=0, x x=x 0 ; x<n C o l \& \& x<x S C R ; x++, x x+=x P e r P i x)\{$

if(solid $[z z][y y][x x]) \quad c c=' s '$;

else \{

if (nveloc [s]==19) ftemp = ffs [0] [zz] [yy] $[\mathrm{xx}]$;

else $\{/$ * 5-22-97; 5-27-97 remove 0.25* below */

ftemp $=(\mathrm{ffs}[0][z z][y y][\mathrm{xx}]+\mathrm{ffs}[1][z z][\mathrm{yy}][\mathrm{xx}]+$ ffs [2][zz][yy][xx]+ffs[3][zz][yy][xx]+ ffs $[4][z z][y y][x x]+$ ffs $[5][z z][y y][x x])$;

$\mathrm{cc}=$ ' ' $\hat{\mathrm{U}}$ ';

for $(k=0 ; k<5 ; k++)\{$

if ( ftemp<f $[\mathrm{k}]$ ) \{

$\mathrm{cc}=\mathrm{c}[\mathrm{k}]$; break;

\}

\}

3

putchar (cc);

\}

putchar (' $\backslash \mathrm{n}$ ') ; 


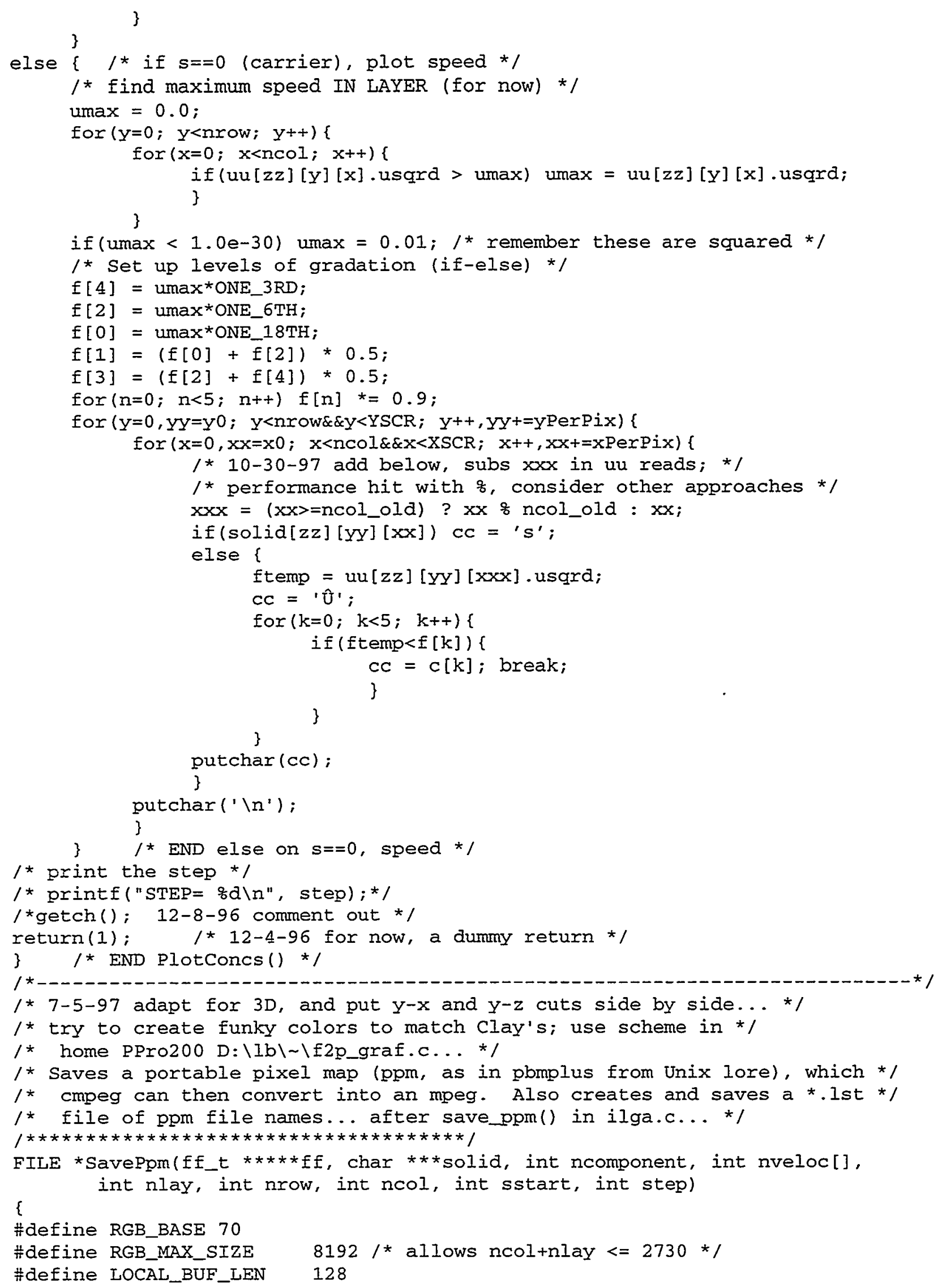


static unsigned char rgb_row[RGB_MAX_SIZE+4] ;

1* for mpeg writes, 3* max ncol */

static int filenum $=0$, firsttime=1, rgb_len;

static FILE * $f p, * b \_l i s t \_f p ;$

int $i, i i, x, y, z$;

unsigned char $r, g, b$;

double c, $c \max =-1.0 \mathrm{e} 200, \mathrm{cmin}=1.0 \mathrm{e} 200$;

double slopel, slope2, delta, chalf, craw, cpt75;

struct tm *ptm;

static int day $=0$, hour $=0 ; /$ because localtime destroys single internal tm *

time_t It;

char buf[LOCAL_BUF_LEN]; $/$ * for filenames * $/$

int xhalf, zhalf;

if (filenum $>=999)$ \{

firsttime $=1$;

filenum $=0$;

if (b_list_fp) fclose(b_list_fp);

\}

if (firsttime)

/* ppm format has thre byte rgb values for each pixel *I

time (\&lt);

ptm = localtime $(\& I t)$;

1 * 9-12-94 for list file */

day $=$ ptm->tm_mday, hour $=$ ptm->tm_hour;

sprintf (buf, "B\%02d\%02d.Ist", day, hour) ;

buf [LOCAL_BUF_LEN-1] = 0;

b_list_fp $=$ fopen (buf, "w");

firsttime $=0$;

\}

/* 9-27-97 moved out of firsttime block */

rgb_len $=($ ncol + nlay +1$) * 3$;

/ * 7-5-97 allow placing $y-x$ and $y-z$ cuts side-by-side */

xhalf $=$ ncol $>1 ;$ zhalf $=$ nlay $>1$;

/* find the maximum and minimum conc values */

1 * NOTE we initialized cmax, cmin in declarations, above */

for $(y=0 ; y<n r o w ; y++)\{$

for $(x=0 ; x<n c o l ; x++)\{$

if (solid[zhalf] [y] $[\mathrm{x}])$ continue; $/ * 7-12-97 * /$

for $(i=0, c=0.0 ; i<$ nveloc [sstart $] ; i+t)\{$

$c+=f f[$ sstart] [i] [zhalf] $[\mathrm{y}][\mathrm{x}]$;

\}

if $(c>=c \max ) \quad c \max =c$;

if $(c<=c \operatorname{cmin}) \operatorname{cmin}=c$;

\}

for $(z=0 ; z<$ nlay; $z++)\{$

if (solid[z] [y] [xhalf]) continue; $1 * 7-12-97 * /$

for $(i=0, c=0.0 ; i<$ nveloc [sstart] ; $i++)\{$

$c+=$ if[sstart][i][z][y][xhalf];

\}

if $(c>=c \max ) \quad c \max =c$;

if $(c<=c \operatorname{cmin}) \mathrm{cmin}=\mathrm{c}$;

\} \} 
1 * Prevent funny colors due to small oscillations */

if $(\mathrm{cmin}<0.0) \mathrm{cmin}=0.0$;

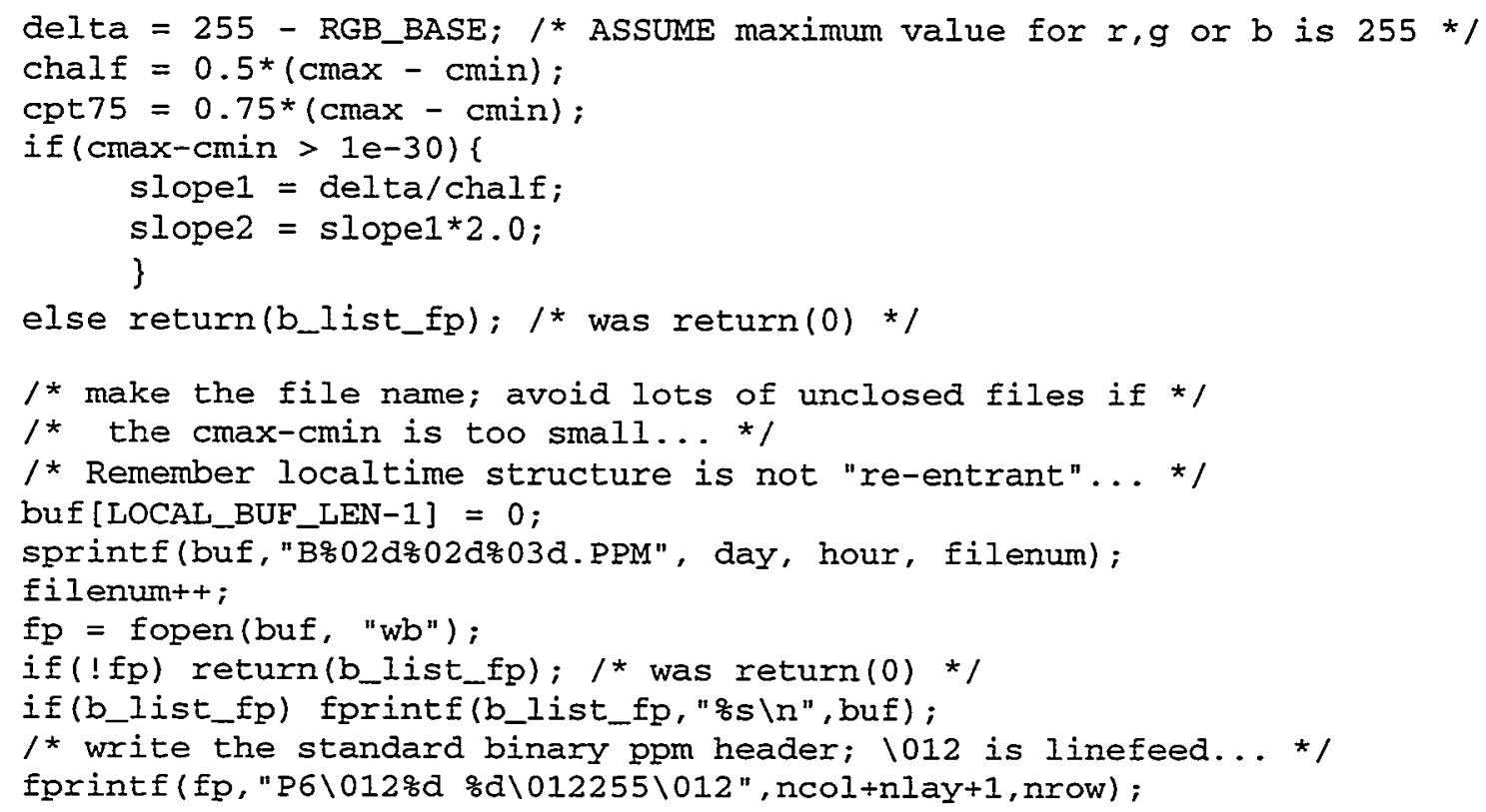




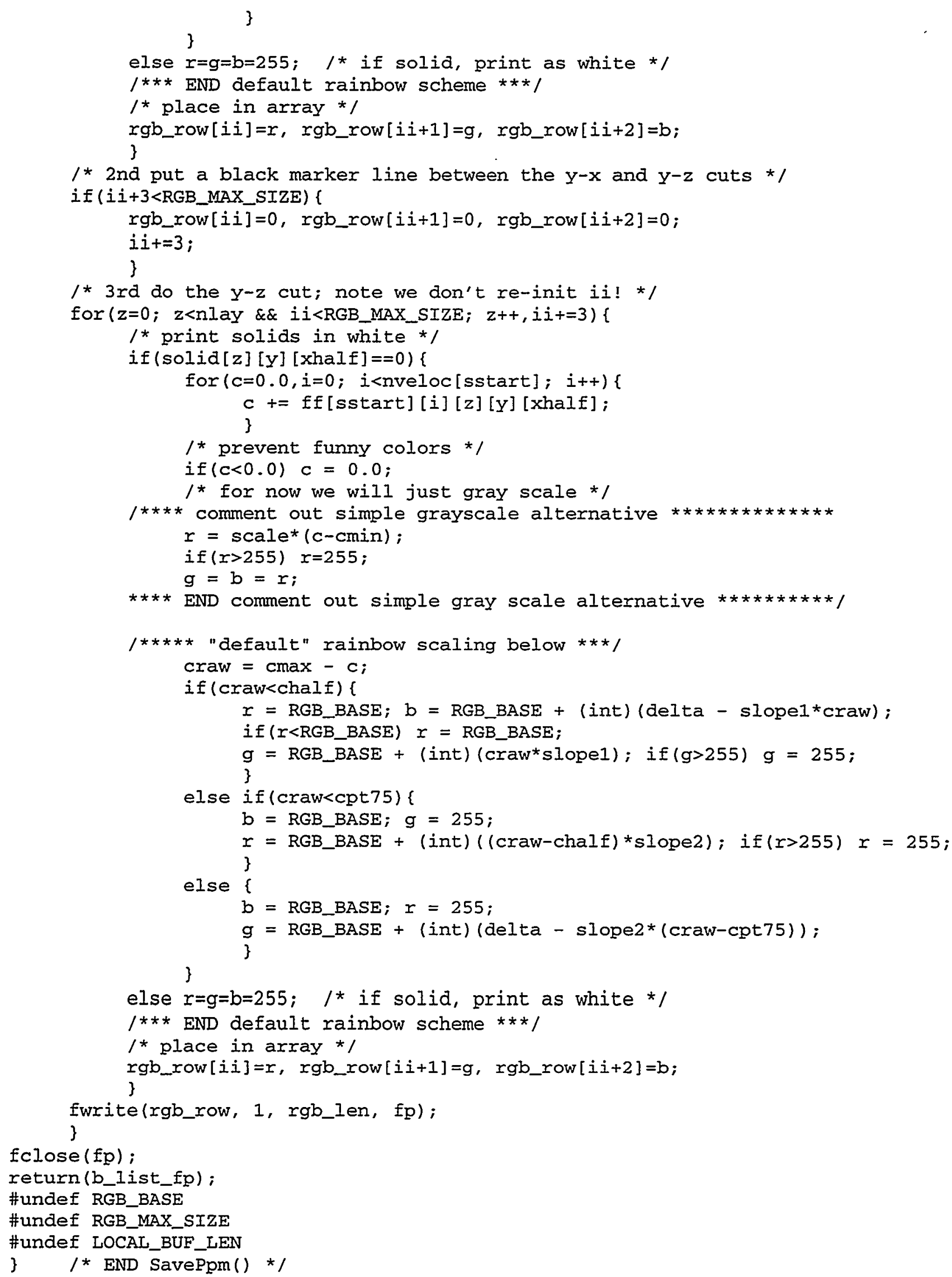




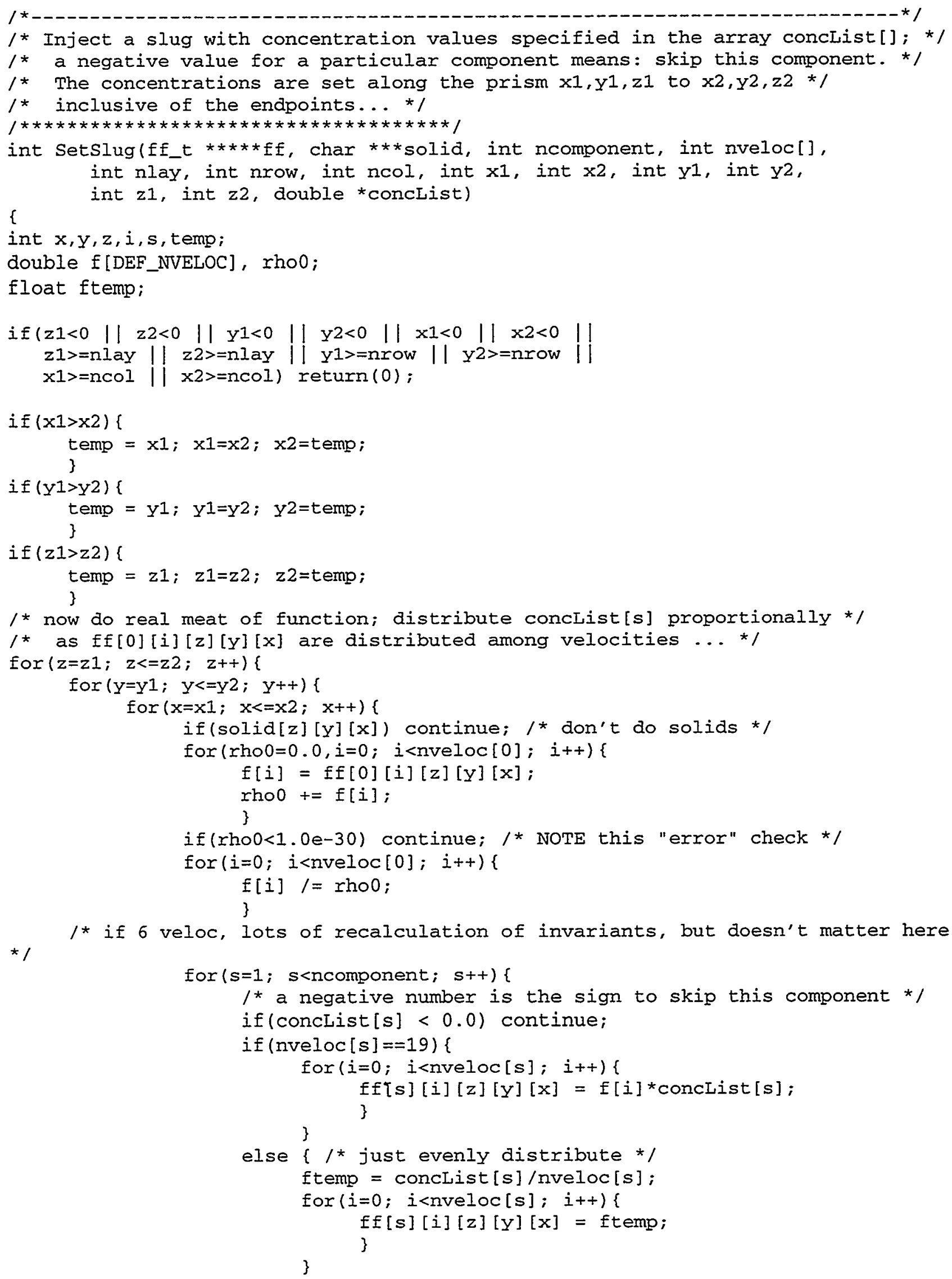




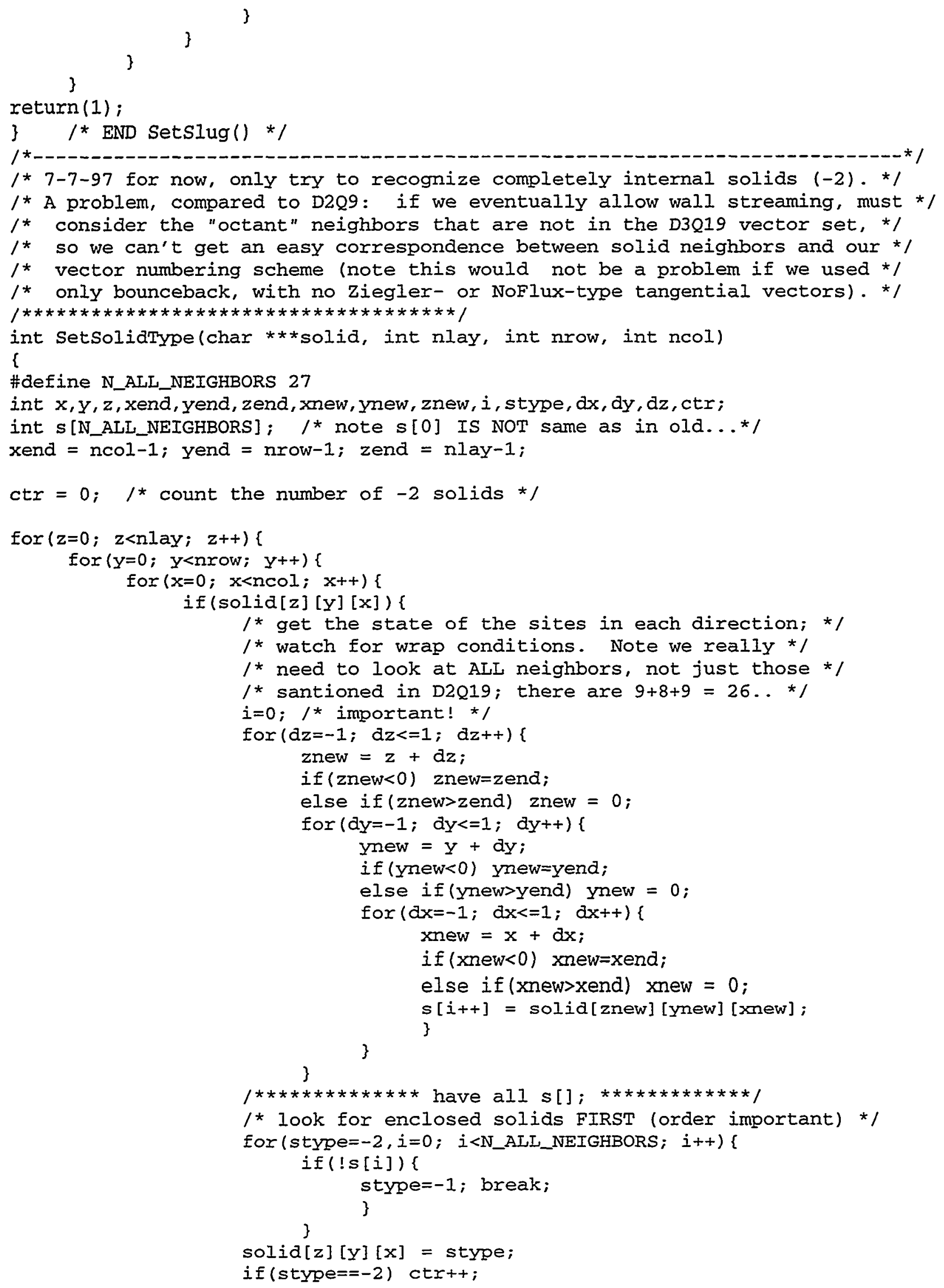




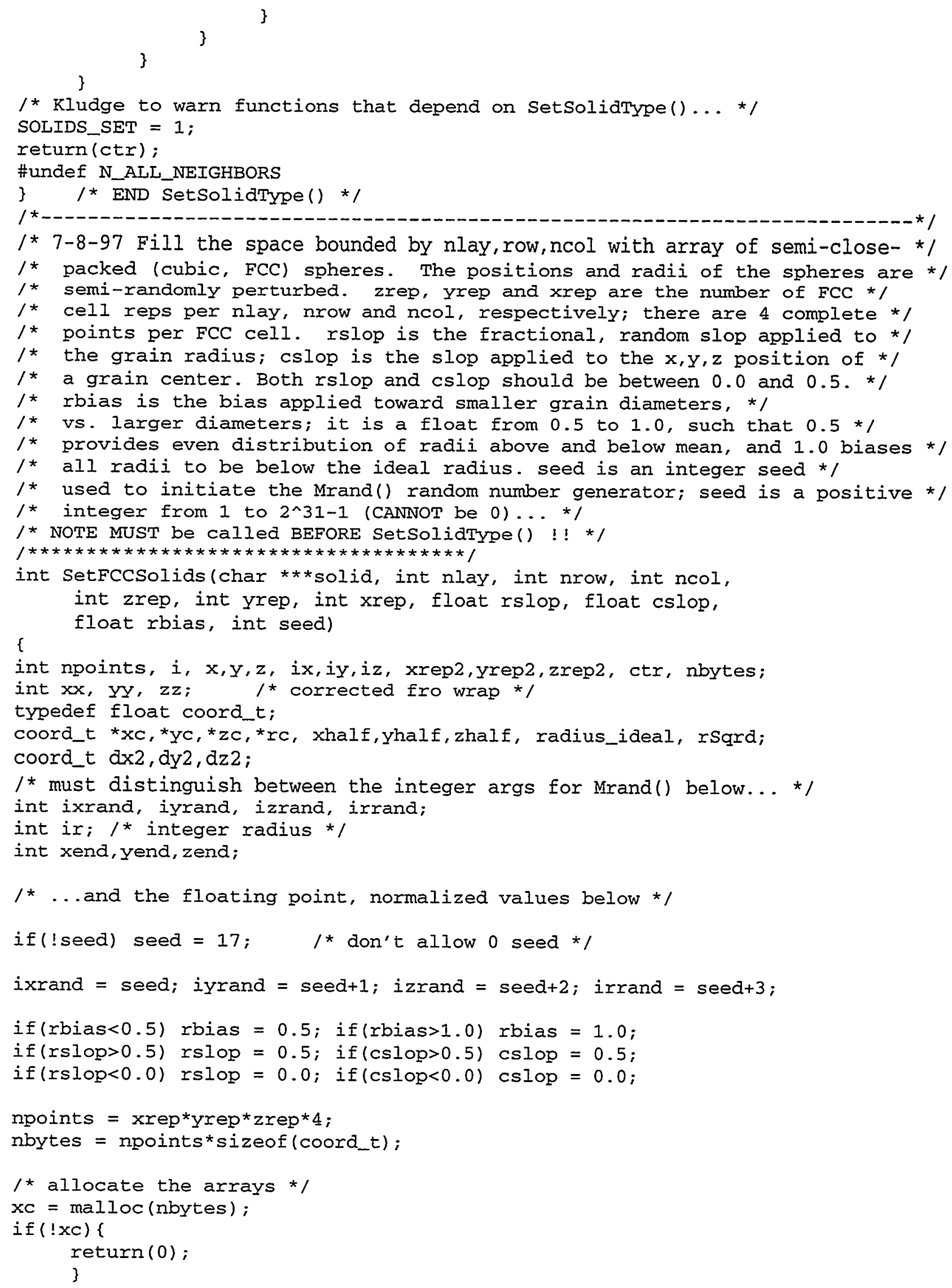




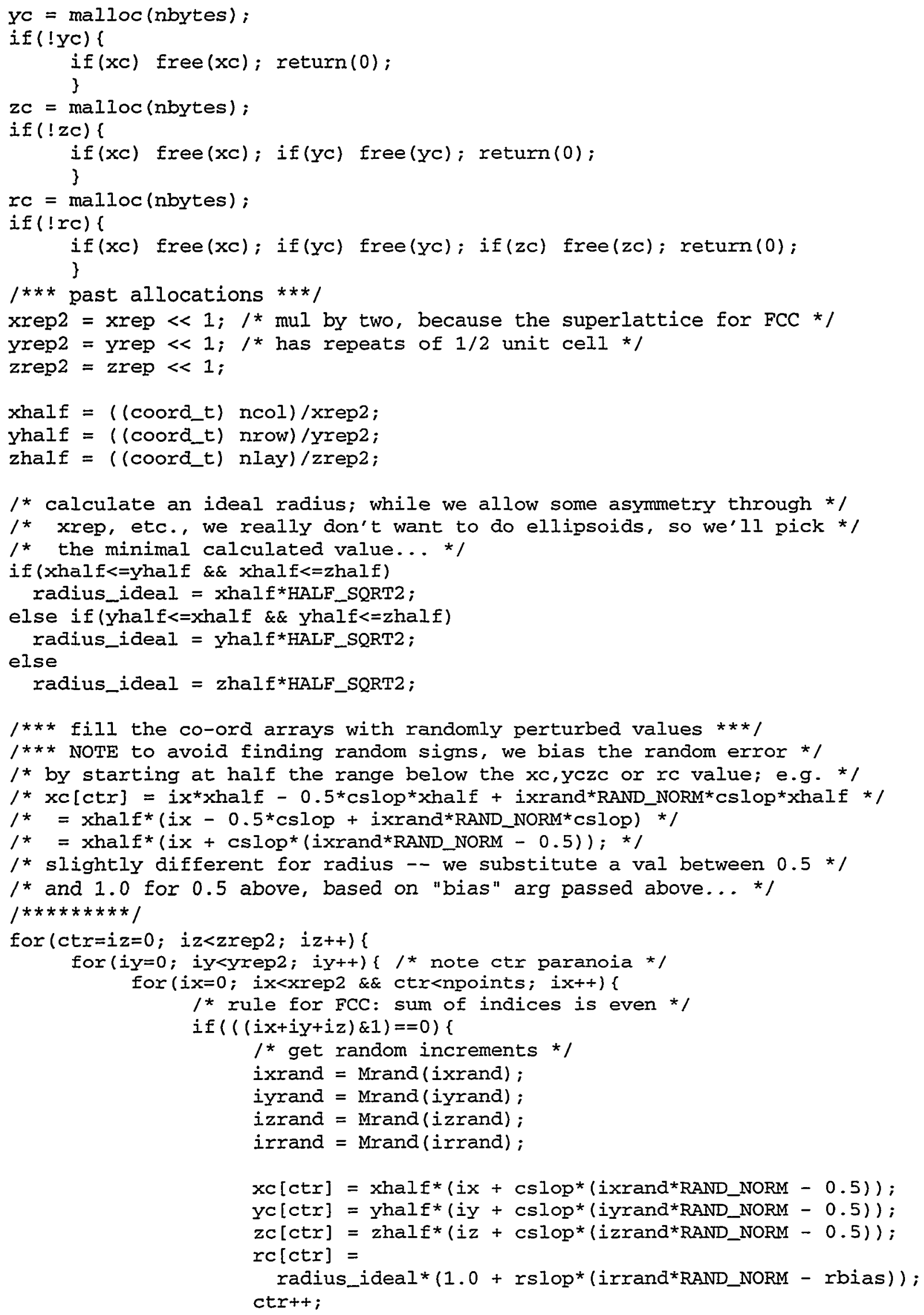




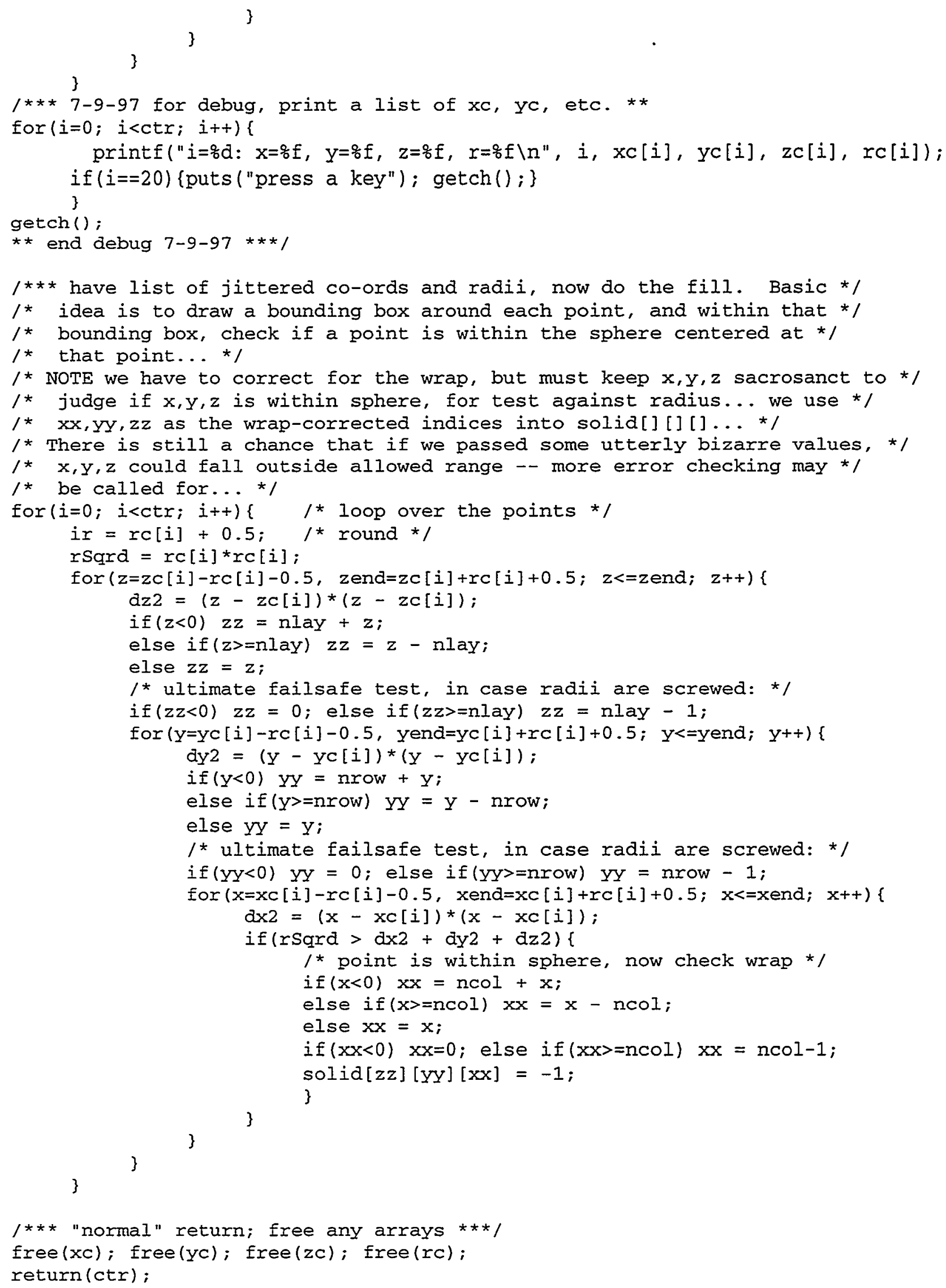


\} $\quad /$ END SetFCCSolids () */

/*---

/* 6-13-97 adapted from home GW2K PPro200 c: \ilga lasm_lga.asm; */

/* uses GGUBS type PRNG; avoids interger division by using * /

/* "simulated" division described in Knuth "Seminumerical..." */

/* NOTE unlike rand(), does not require seed - - always gives */

$1 *$ back the next arg in sequence after $z$. Usual procedure is */

/* to keep reusing $z$, so $z$ itself should NOT be altered between */

/* calls. Iower bits are supposedly nice and random. */

1* Unix, Iinux or alpha NT users: the PRNG is: */

* new $\mathrm{Z}=(16807 * \mathrm{Z})$ MOD 0x7FFFFFFF, sometimes called "GGUBS". The */

1 * range is obviously 1 to 0x7FFFFFFF, so don't use $\mathrm{z}=0$. The */

/* modded value is $2^{\wedge}\{31\}-1$, a Mersenne prime number. If you have */

/* fast integer division, code can be simplified; if you have 64-bit */

$1 *$ integers, code is trivial. If your system has random(), it is */

/* probably at least as good a substitute (MS VC++ is supplied with */

$1 *$ only rand(), which gives back just 15 bits). */

/* NOTE if you subs a different random number generator, you should change */

$1 *$ MRAND_RANGE to the new generator's range! */

/*************************************/

int Mrand (int $z$ )

\{

_asm

\{

mov eax, 16807

mul z

shld edx, eax, 1

and eax, 7FFFFFFFH

add edx, eax

jns replacez

sub edx, 7FFFFFFFH

replacez: mov eax, edx

; return in eax..

\}

\} $/ *$ compiler may complain about no return value -- it is implicitly in eax */

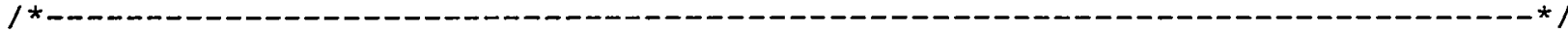

/* 5-21-97 from Ib01.C, modify to use UxAvg() and UyAvg() */

/* 2-16-97 comment out Inc adjustment, just reset Body forces */

$l^{*}$ and return -1 , so main can turn off access to this function... */

/* 2-15-97 add code to chech gotHit and do clamping */

/* 2-4-97 adjust the gravity (xbody, ybody) if required to keep */

$1^{*}$ below or at a constant average speed on the Ux and UY 1 ines... */

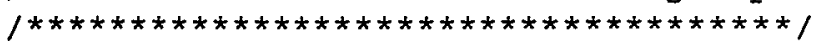

int Adjustgrav(struct veloc_t * const*const* uu, char * const*const* solid,

int nlay, int nrow, int ncol, struct gravity * const gp)

\{

double ux, uy, uz;

double uxLow, uyLow, uzLow;

static int stepcounter $=0$, gotHit $=0$, done=0;

static double ExSum=0.0, fySum=0.0, fzSum=0.0, uxSum=0.0, uysum=0.0, uzSum=0.0；

double uRatio;

int $d t$;

if (done==-1) return $(-1) ; /$ * 2-16-97 failsafe */

uxLow $=0.99 *$ gp->uxLimit; 


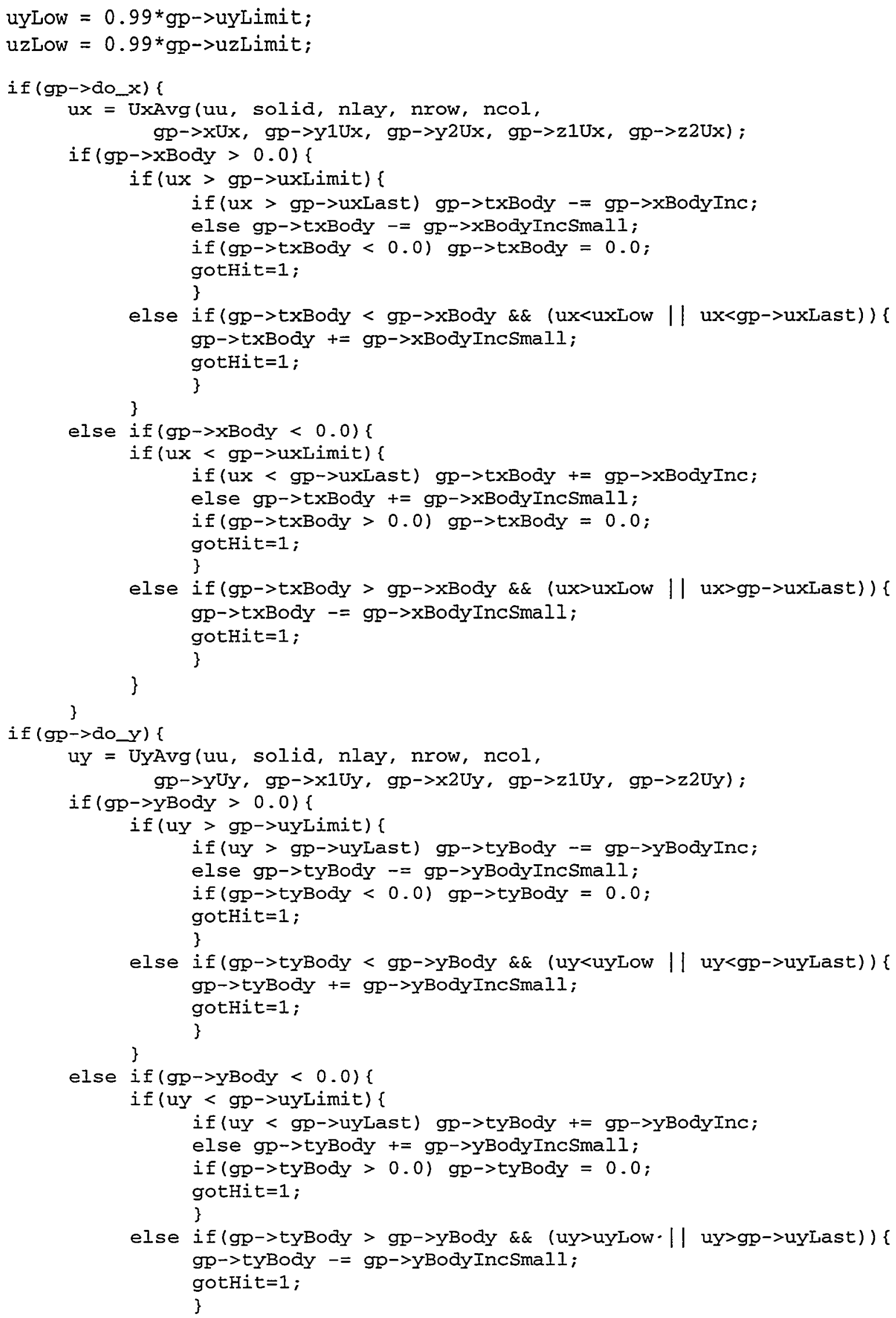




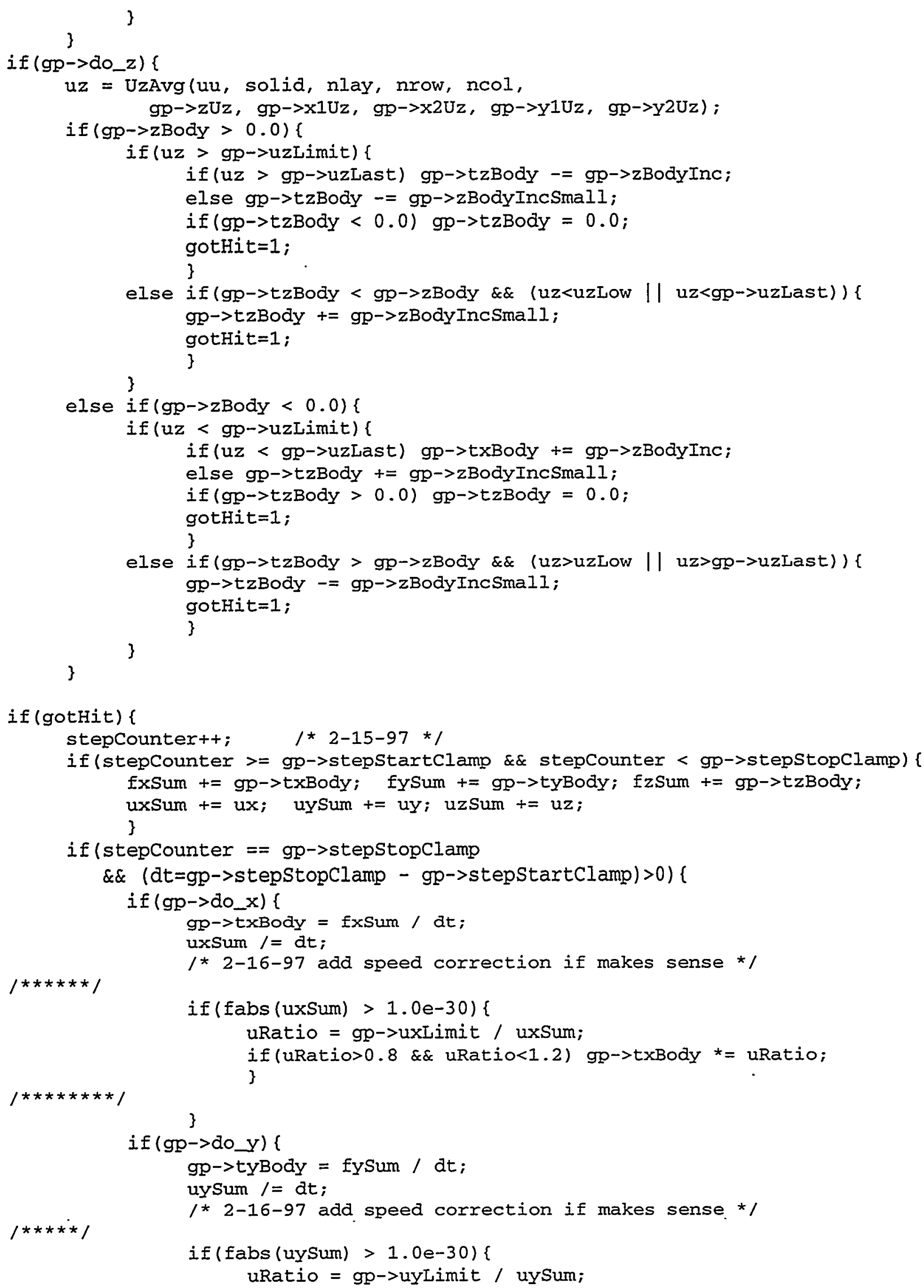




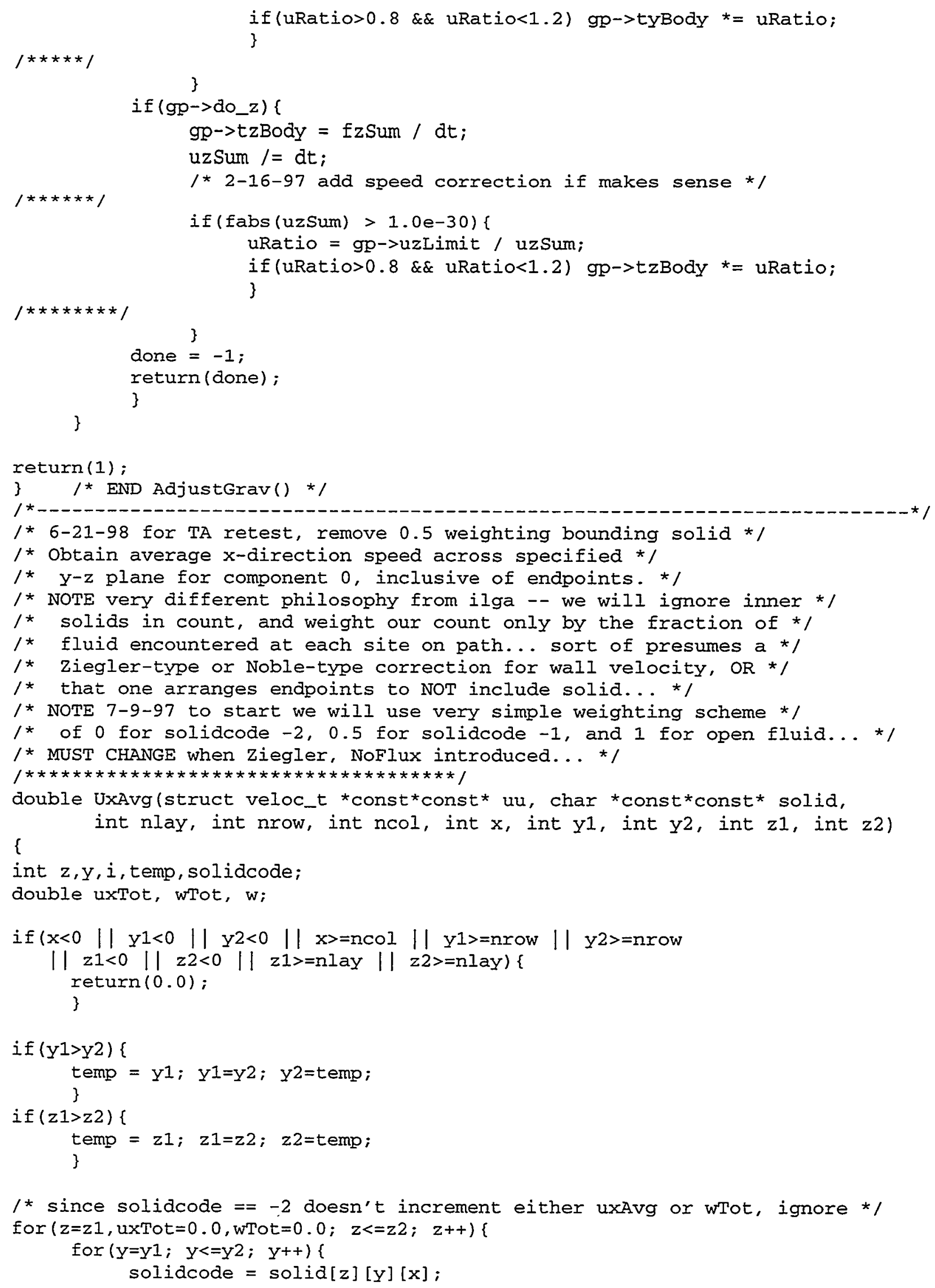




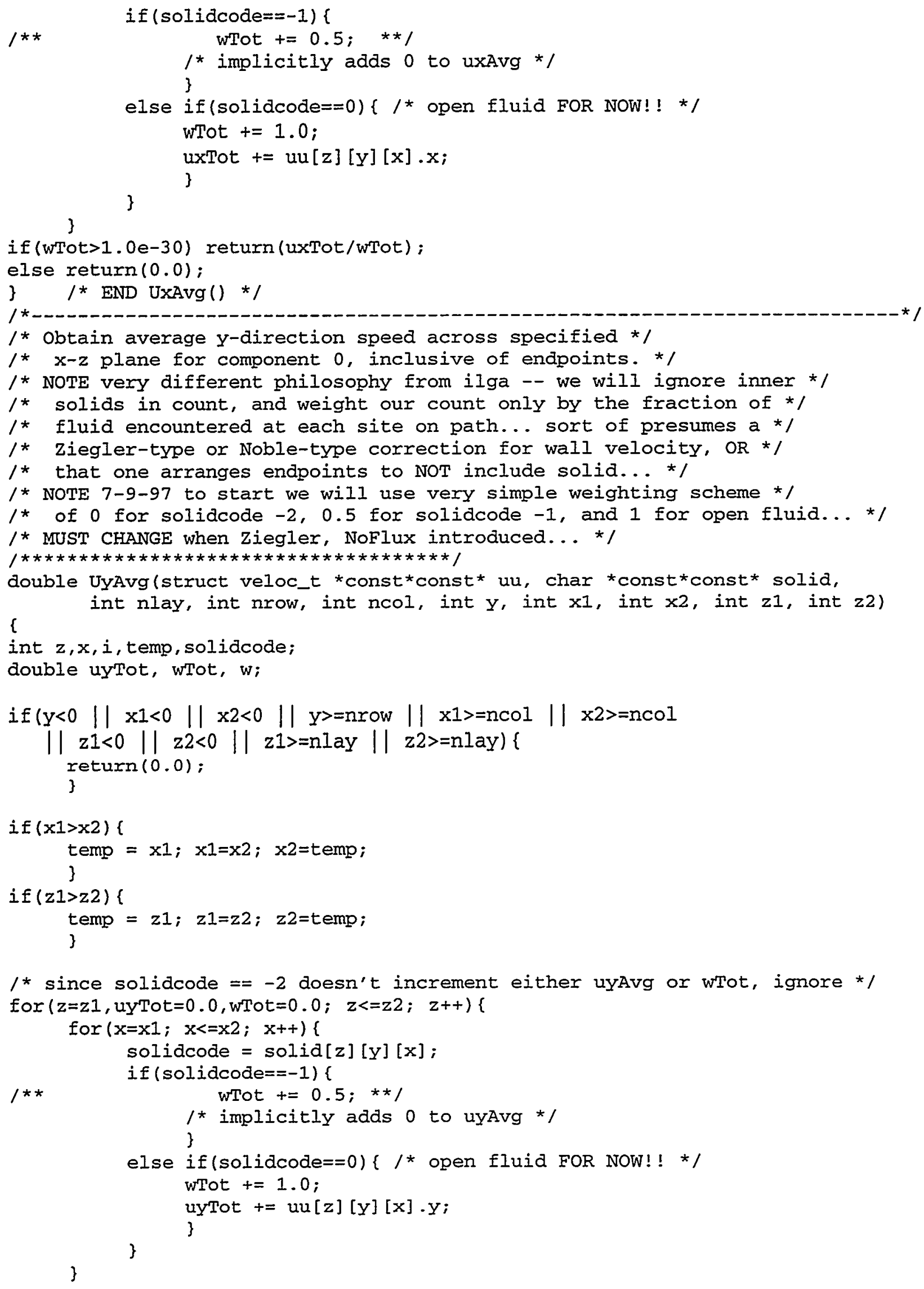


if (wTot>1.0e-30) return(UyTot/wTot);

else return (0.0);

3 $/ *$ END UyAvg ()$* /$

$/ *$ Obtain average $z$-direction speed across specified */

/* $x-y$ plane for component 0 , inclusive of endpoints. */

1 * NOTE very different philosophy from ilga -- we will ignore inner */

$1 *$ solids in count, and weight our count only by the fraction of *

$1 *$ fluid encountered at each site on path... sort of presumes a */

/* Ziegler-type or Noble-type correction for wall velocity, OR */

$1 *$ that one arranges endpoints to NOT include solid... */

1 * NOTE 7-9-97 to start we will use very simple weighting scheme */

$l^{*}$ of 0 for solidcode $-2,0.5$ for solidcode -1 , and 1 for open fluid... *

$1 *$ MUST CHANGE when Ziegler, NoFlux introduced... */

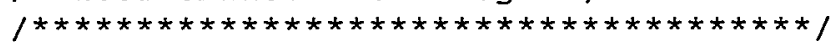

double UzAvg(struct veloc_t *const*const* uu, char *const*const* solid, int nlay, int nrow, int ncol, int $z$, int $x 1$, int $x 2$, int $y 1$, int $y_{2}$ )

\{

int $x, y, i$, temp, solidcode;

double uzTot, wTot, w;

if $\left(z<0|| y 1<0|| y^{2}<0|| z>=n l a y \| y 1>=\right.$ nrow $\| y^{2}>=$ nrow

|| $x 1<0|| \times 2<0|| x 1>=n c o l|| \times 2>=n \operatorname{col})\{$ return (0.0);

\}

if $\left(y 1>y^{2}\right)\{$

temp $=\mathrm{y} 1 ; \mathrm{y} 1=\mathrm{y}^{2} ; \mathrm{y} 2=$ temp;

\}

if $(x 1>x 2)\{$

temp $=\mathrm{x} 1 ; \mathrm{x} 1=\mathrm{x} 2 ; \mathrm{x} 2=$ temp;

\}

/ since solidcode $==-2$ doesn't increment either uzAvg or wTot, ignore * for $(y=y 1$, uzTot $=0.0$, wTot $=0.0 ; y<=y 2 ; y++)($

for $(\mathrm{x}=\mathrm{x} 1 ; \mathrm{x}<=\mathrm{x} 2 ; \mathrm{x}++)\{$ solidcode $=$ solid $[z][y][x] ;$

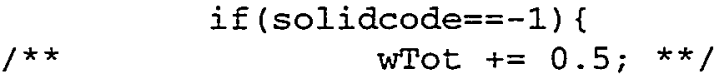

* implicitly adds 0 to uzAvg */

\}

else if(solidcode==0)\{/* open fluid FOR NOW!! */

wTot $+=1.0$;

uzTot $+=$ uu $[z][y][x] \cdot z$;

\}

\}

if (wTot>1.0e-30) return(uzTot/wTot);

else return $(0.0)$;

3 $\quad /$ END UzAvg ()$* /$

$1 * \ldots+\ldots$

/* 9-26-97 NOTE for the JB code, AFTER Recycle() we use XSetConcJB(), */

$1 *$ (below) NOT these funcs! */

/* Set the concentrations to the values specified in the array concList[], */

$l^{*}$ along the plane of constant Y... similar to HSetConc ( $)$ in $2 \mathrm{D}$ models... * $/$

/* a negative value for a particular component means: skip this component. */

1 * The concentrations are set along the plane with opposite corners */ 
$1 * x 1, y, z 1$ to $x 2, y, z 2$, inclusive of the endpoints...*

/* 7-11-97 redesign to: scale */

1 * 6-vector to the corresponding cartesian vactors in 19-vector carrier... */

$1 *$ this method should prevent the bleed-through on edges we possibly got */

/* with some of the fracture $x$ (intersection) calcs done in april 97... */

/*************************************/

int YSetConclff_t $* * * * *$ const ff, char $* * *$ const solid,

int ncomponent, int nveloc[], int nlay, int nrow, int ncol,

int $y$, int $x 1$, int $x 2$, int $z 1$, int $z 2$, double * const conclist)

\{

int $x, z, i, s, t e m p, i s_{-} v 19$, is_v6, nvel;

1 * 7-11-97 the "is_*" above test is any of the solutes are 19 or 6 vector */

$1 *$ types; would be more efficient done just once outside, but seems safer */

1* to do this simple test internally, since it will be dwarfed by fetching */

$l^{*}$ and computation time in rest of function, and we may some day reallocate */

1 * a 19-vector solute among 6 vectors after equilibration.... */

double $f\left[D E F \_N V E L O C\right]$, rhoo, conc;

double fv6[6], rho0v6; $/ * 7-11-97$, for 6-vector methods */

double inv_rhoo, inv_rho0v6;

if $(y<0|| x 1<0|| x 2<0|| y>=$ nrow ||$x 1>=n c o l|| x 2>=n c o l$

|| $z 1<0|| z 2<0|| z 1>n l a y|| z 2>n l a y)$ return $(0)$;

if $(x 1>x 2)$ i

temp $=x 1 ; \quad x 1=x 2 ; x 2=$ temp;

\}

if $(z 1>z 2) \mathfrak{c}$

temp $=z 1 ; z 1=z 2 ; z 2=$ temp;

\}

/* test for need to do 19 or 6 vector scaling */

for (is_v19=is_v6=0, $s=1 ; s<$ ncomponent; $s++$ ) \{

if(concList [s]>=0.0)\{ $/ *$ recall we don't do entries with negative... * /

if (nveloc $[s]==6$ ) is_v6 $=1$;

else if (nveloc[s]==19) is_v19=1;

\}

\}

I* now do real meat of function; distribute concList[s] proportionally */

$I^{*}$ as $\mathrm{ff}[0][\mathrm{i}][\mathrm{z}][\mathrm{y}][\mathrm{x}]$ are distributed among velocities ... */

for $(z=z 1 ; z<z 2 ; z++)\{$

for $(x=x 1 ; x<=x 2 ; x++)\{$

if (solid[z][y][x]==-2) continue; $/$ * don't do internal solids */

if(is_v19) \{ $/$ * if we found ANY solute is 19-vector model: * $/$

for (rhoo $=0.0, i=0 ; i<$ nveloc $[0] ; i++)\{$

$\mathrm{f}[\mathrm{i}]=\mathrm{ff}[0][\mathrm{i}][\mathrm{z}][\mathrm{Y}][\mathrm{x}]$;

rhoo $t=f[i]$;

\}

if $(r h o 0<1.0 e-30)$ continue; $/$ * NOTE this "error" check */

inv_rhoo $=1.0 /$ rhoo;

for $(i=0 ; i<$ nveloc $[0] ; i++)\{$

$f[i] *=$ inv_rhoo;

\}

\}

if (is_v6) $\{/ *$ if ANY solute is a 6-vector model */ 


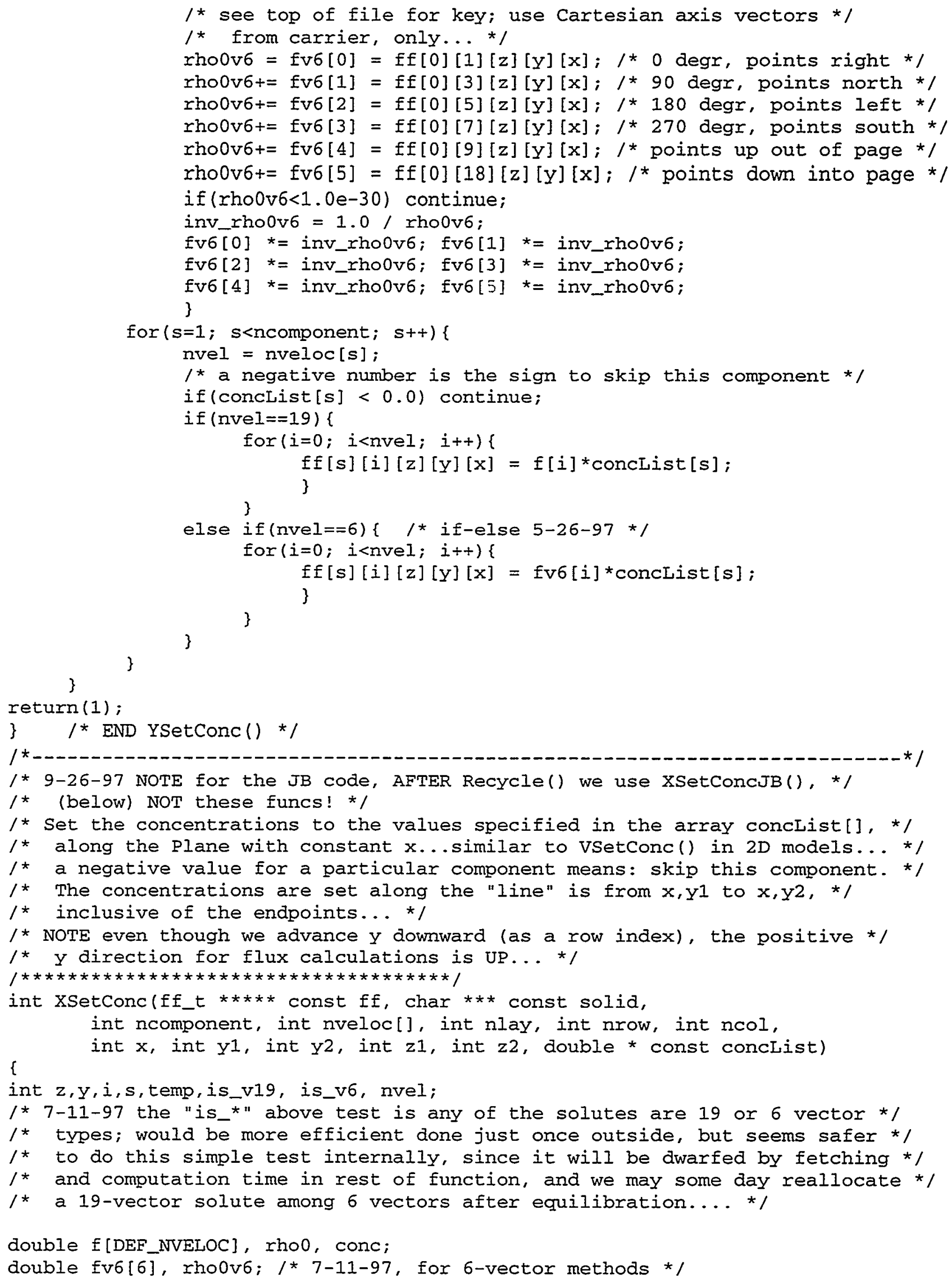


double inv_rhoo, inv_rhoov6;

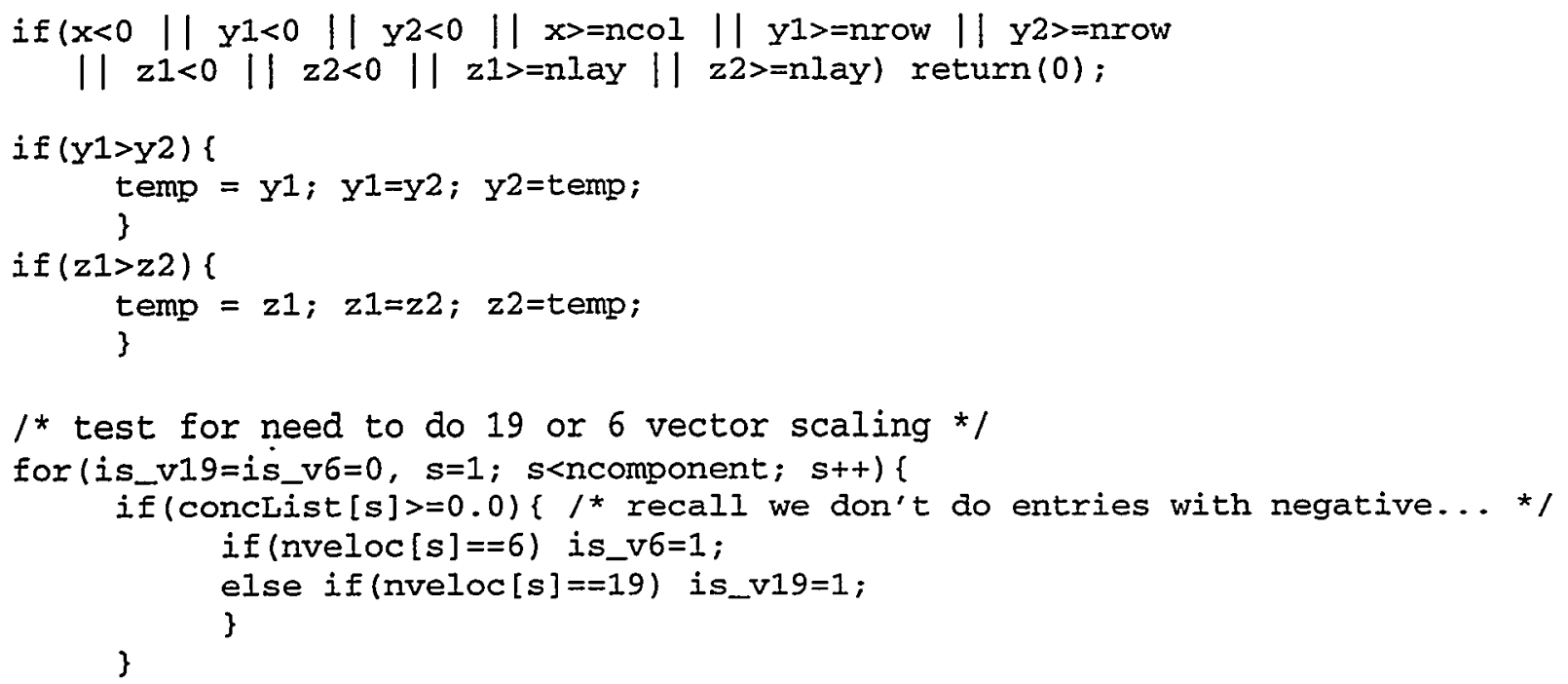




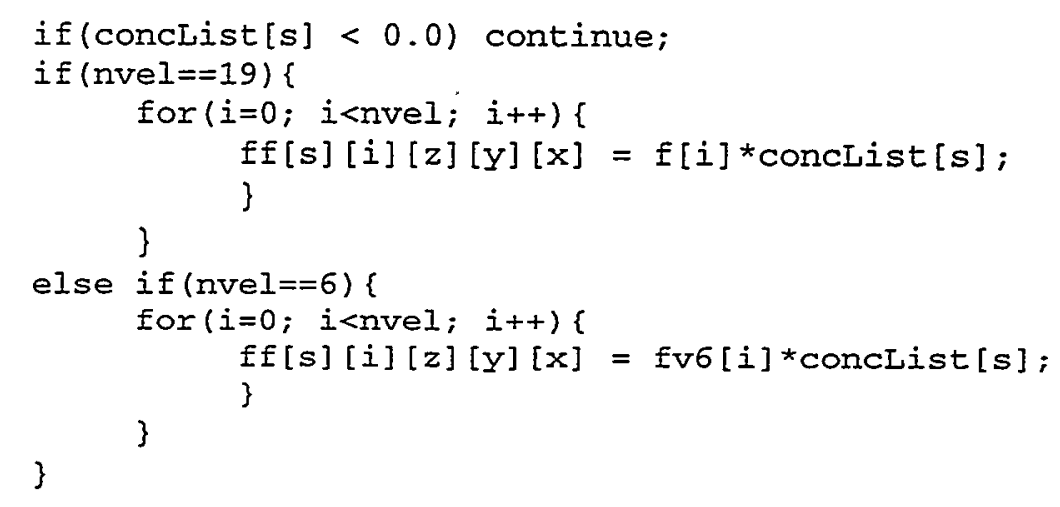




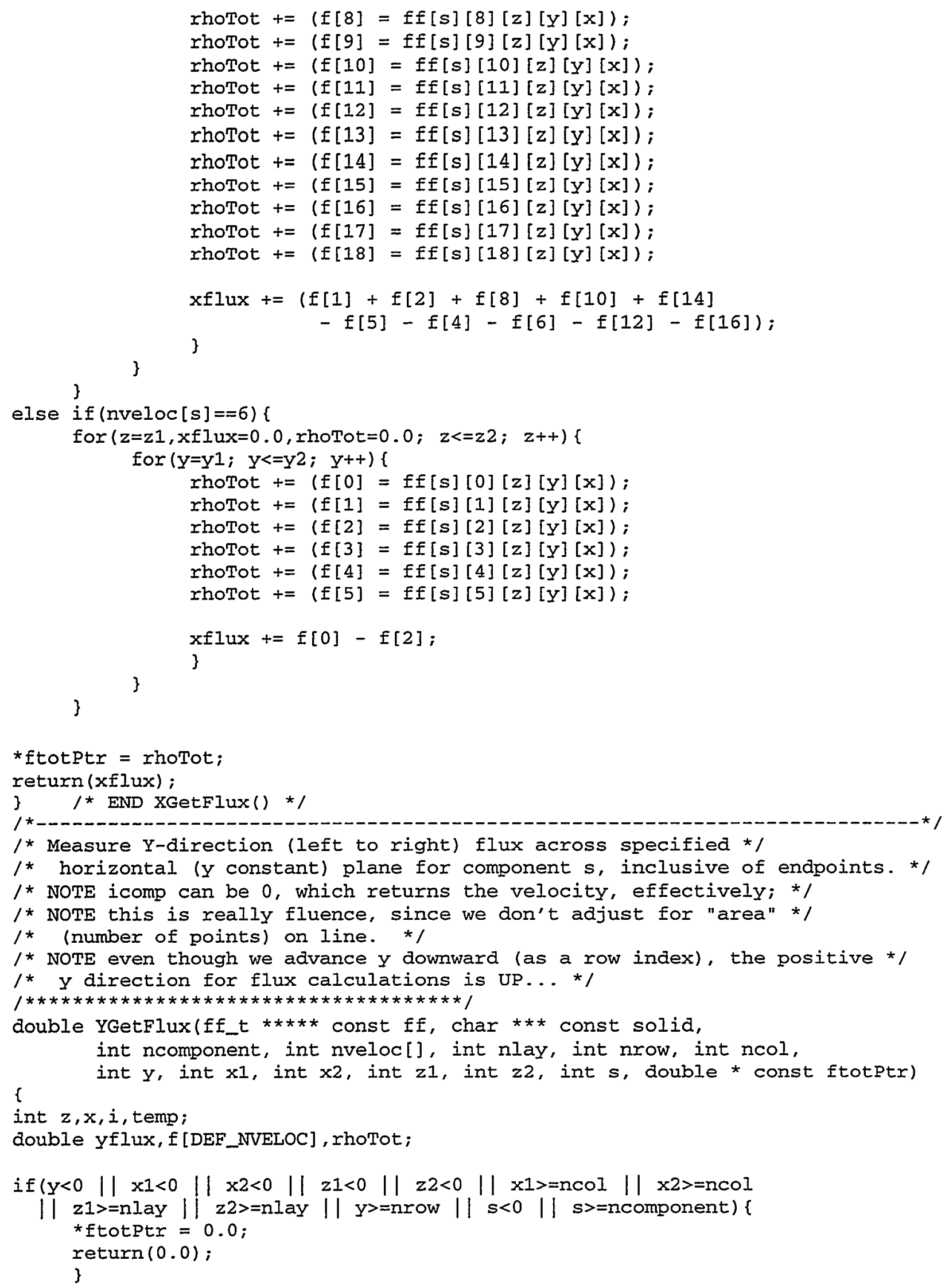




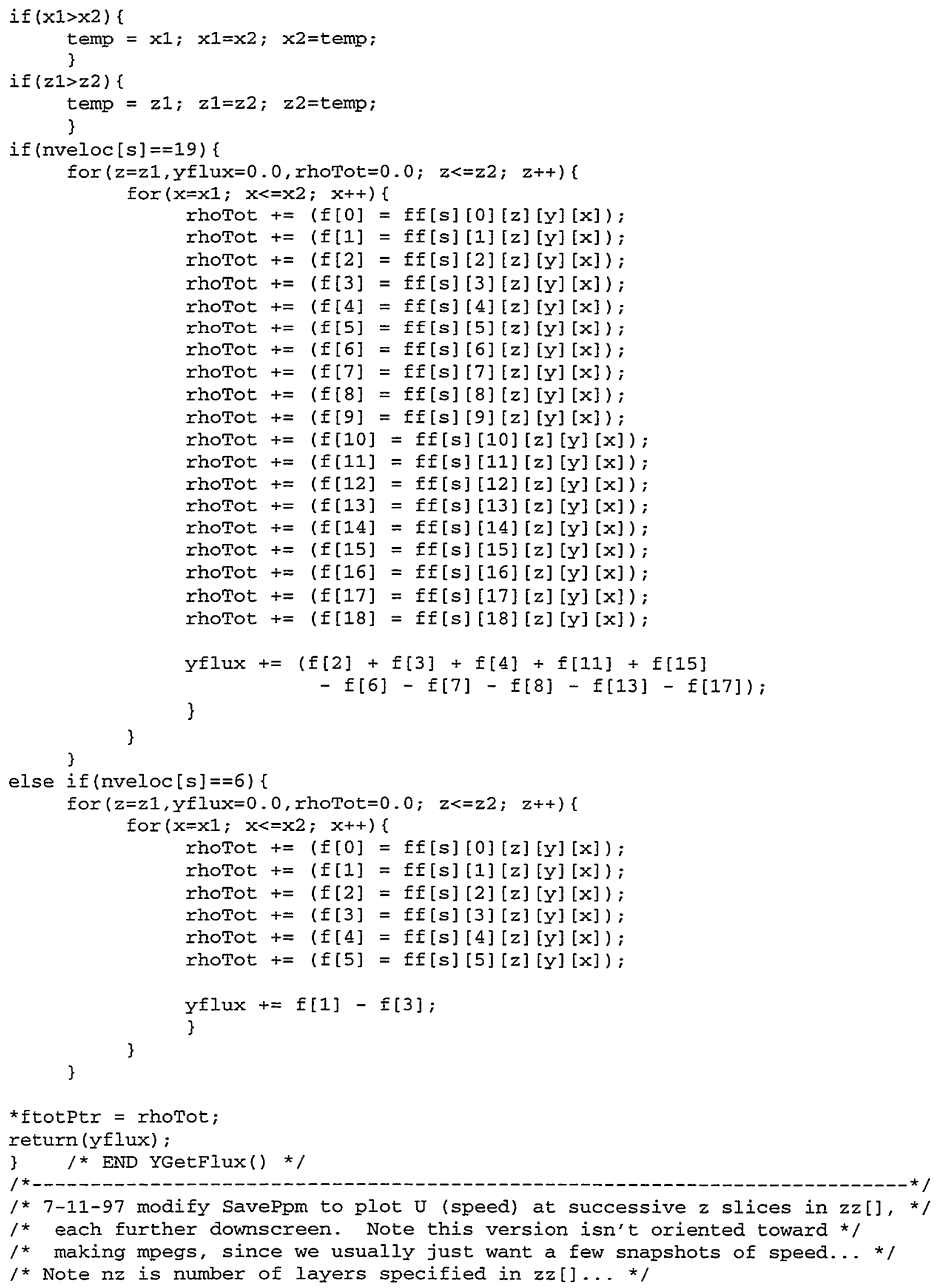




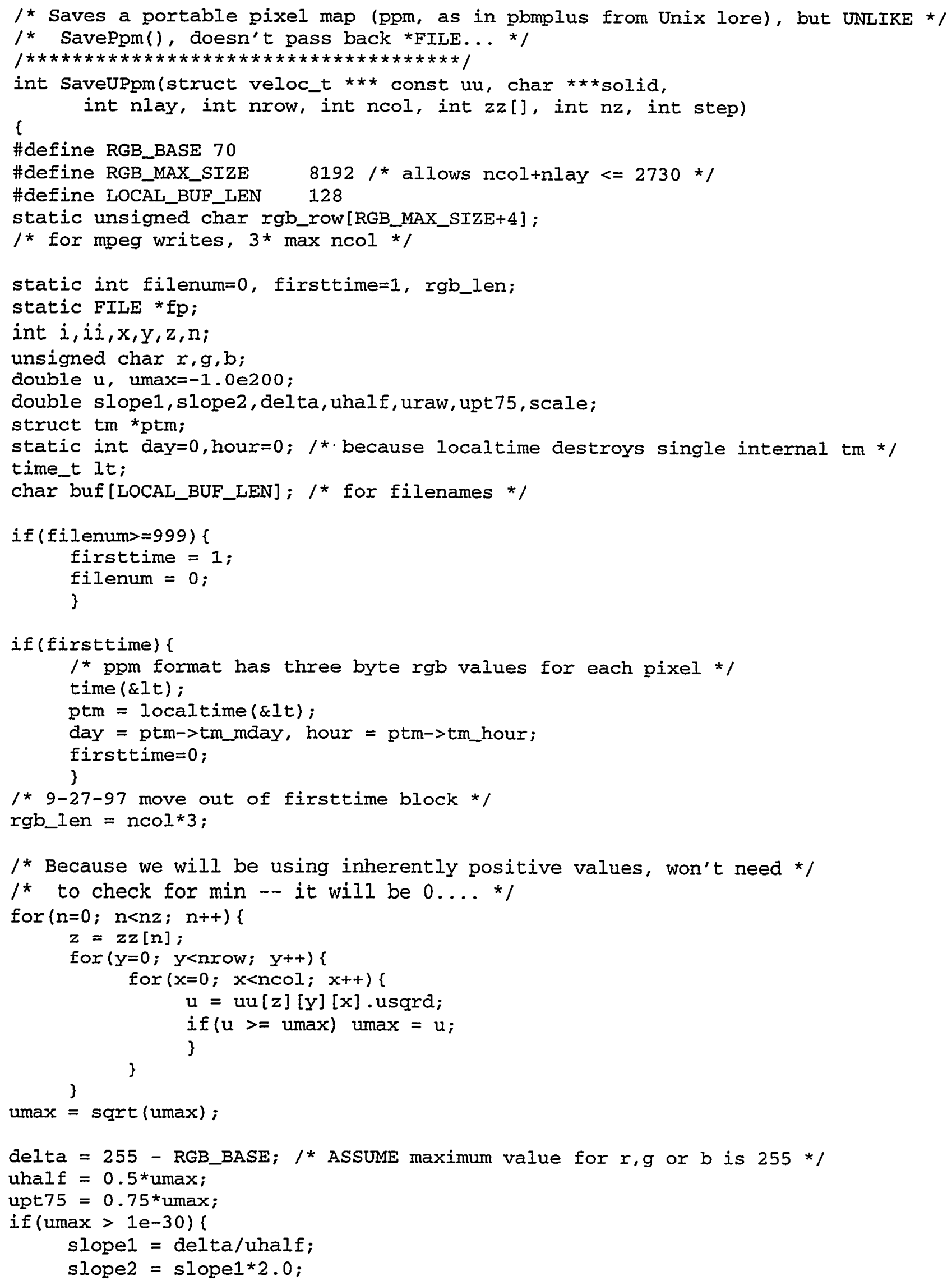




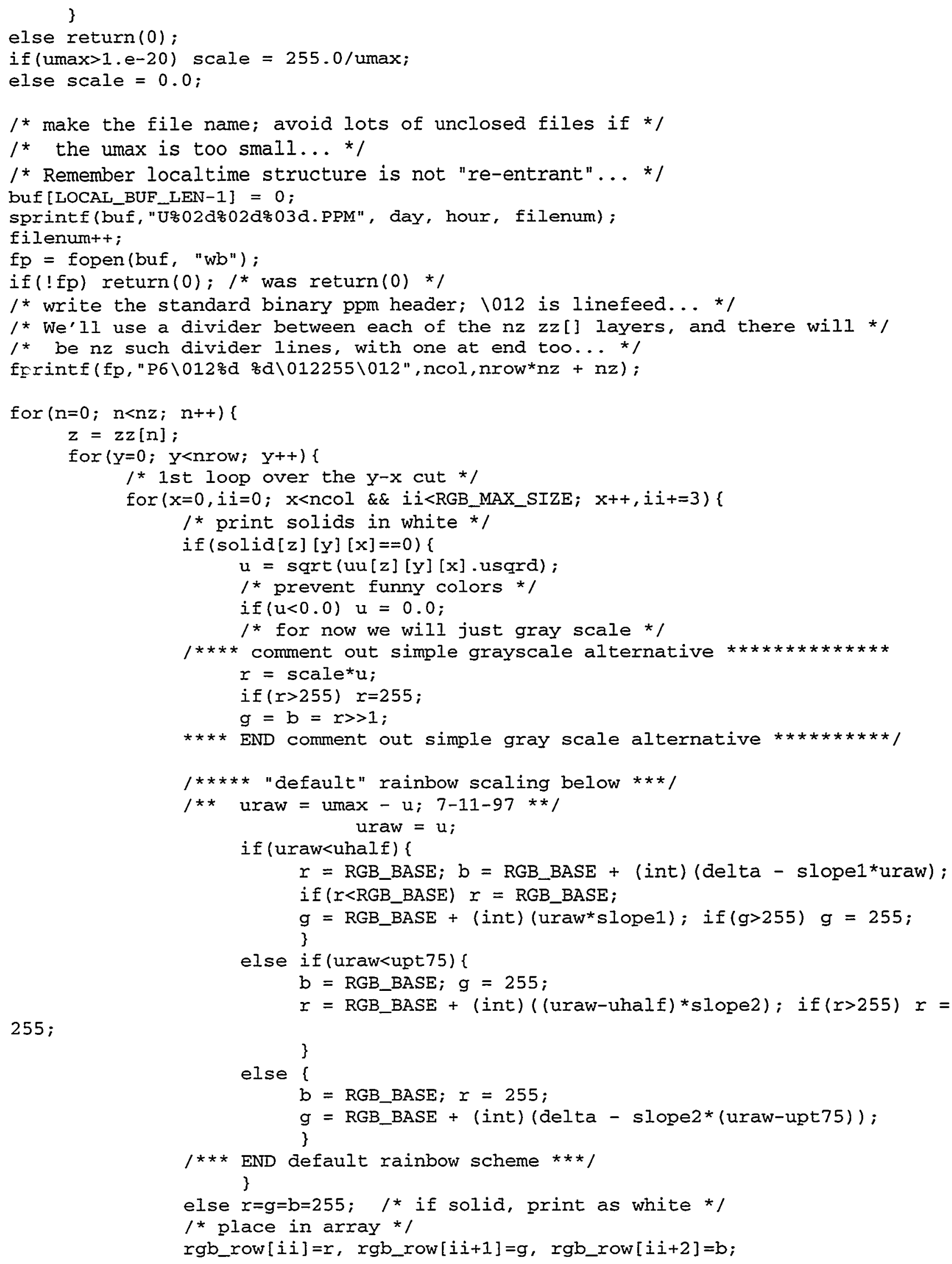




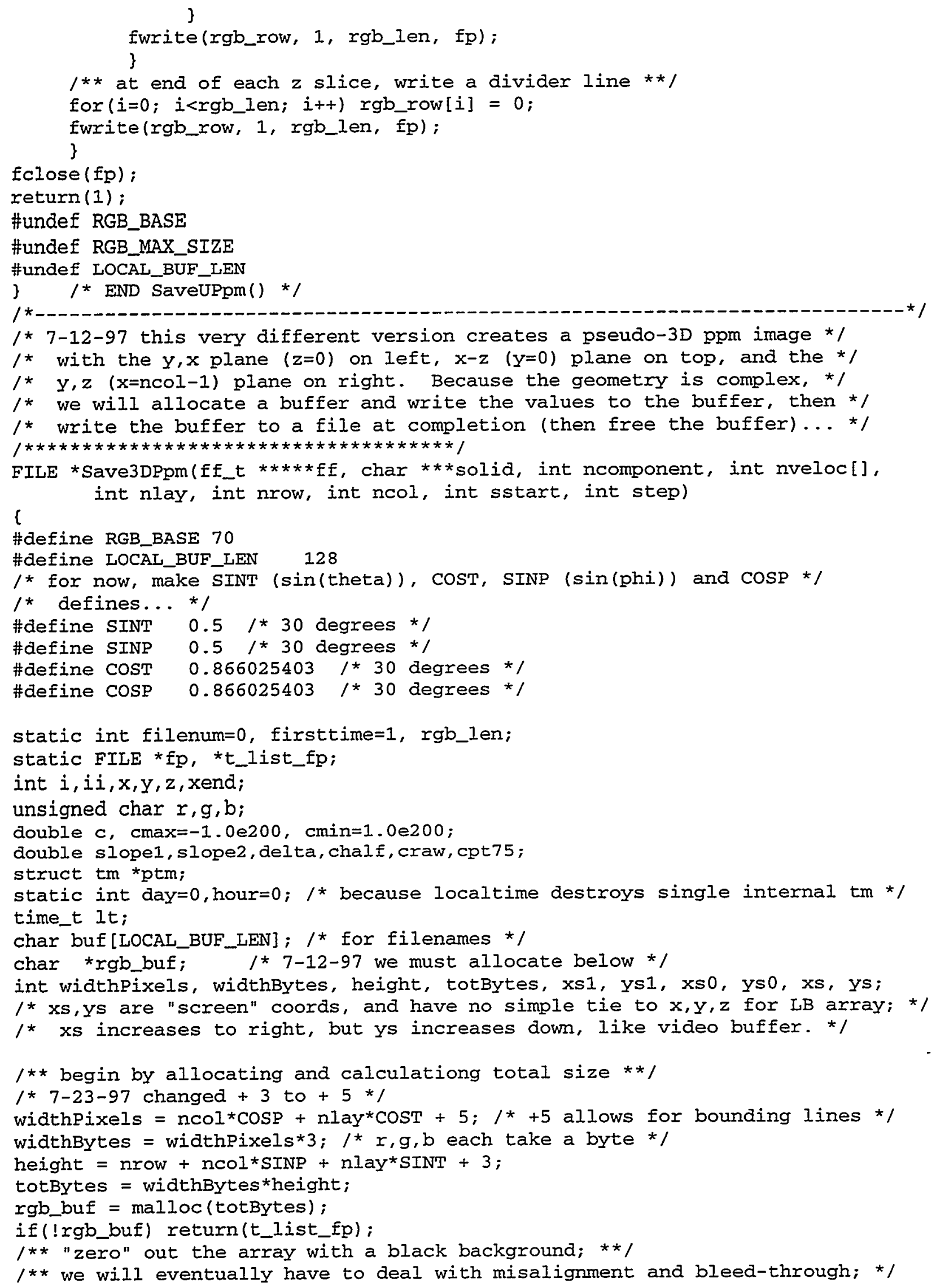




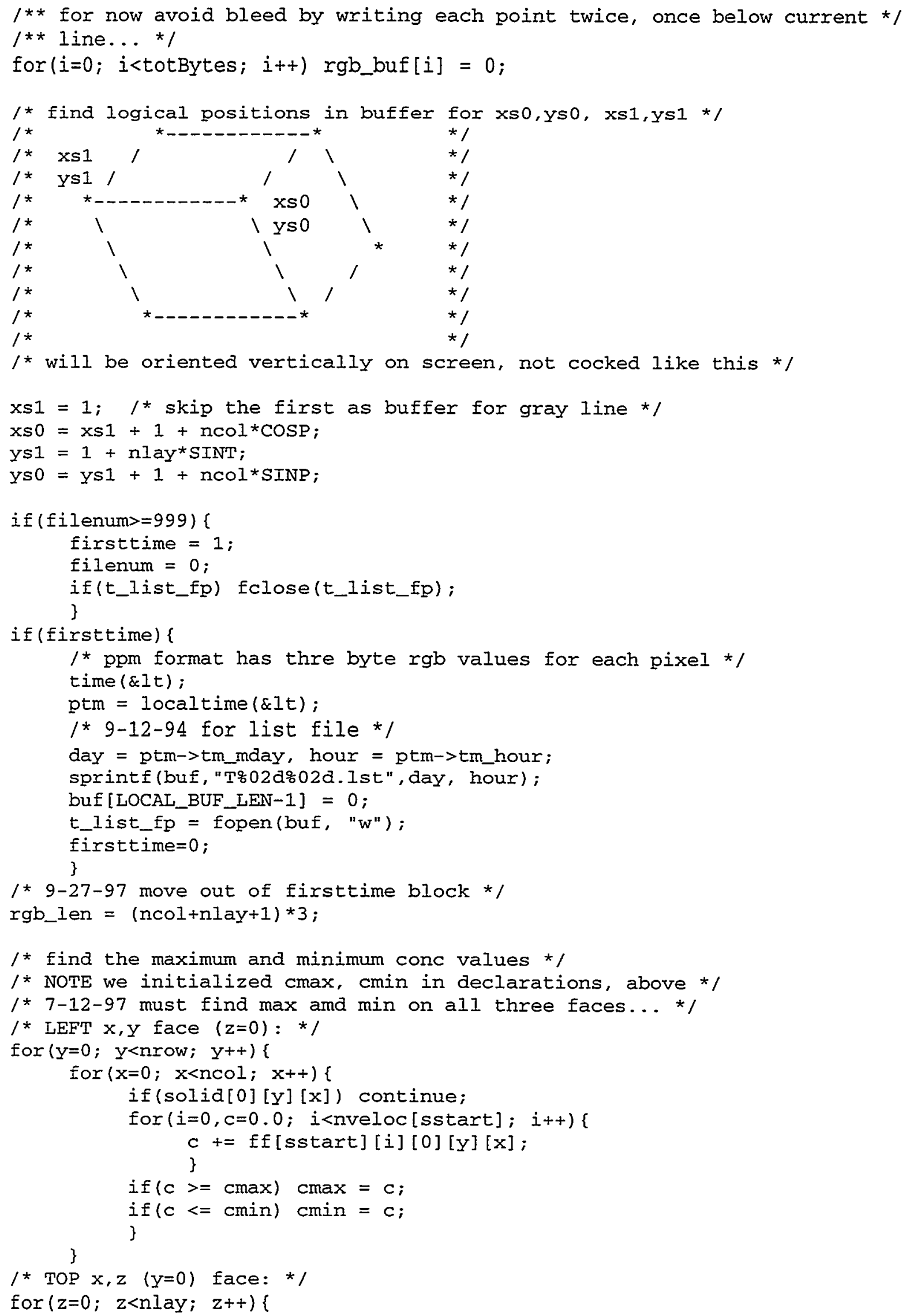




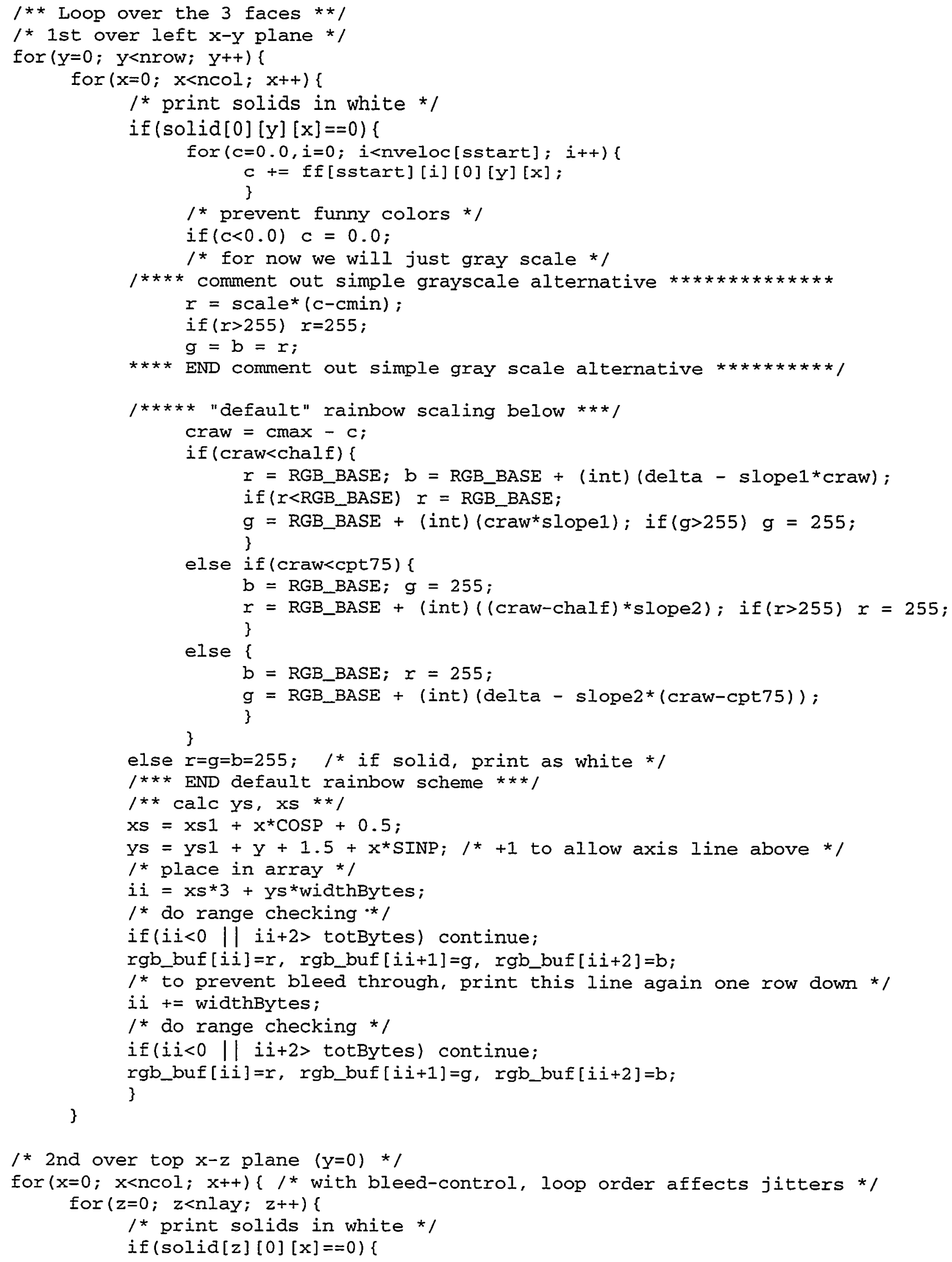




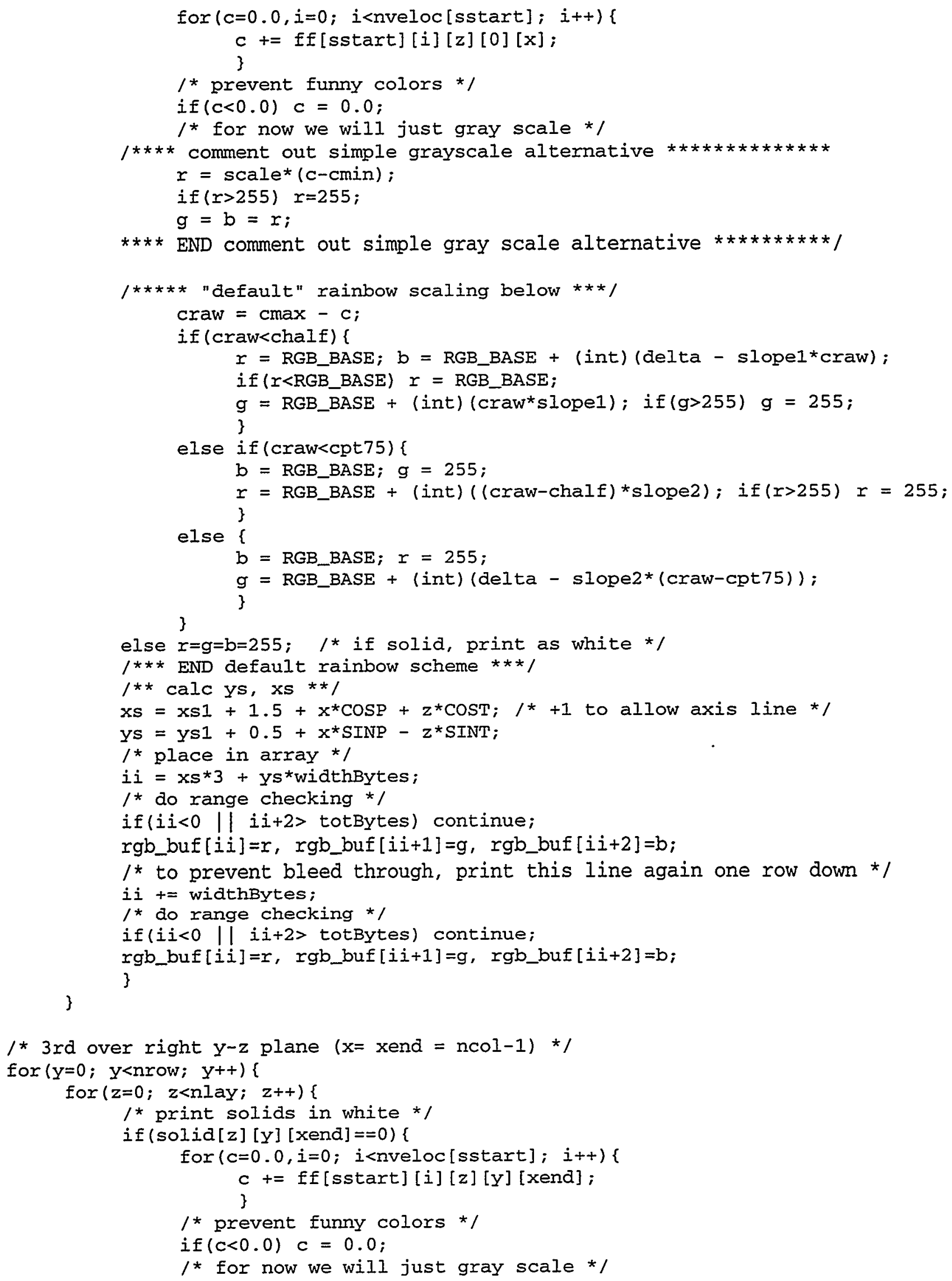




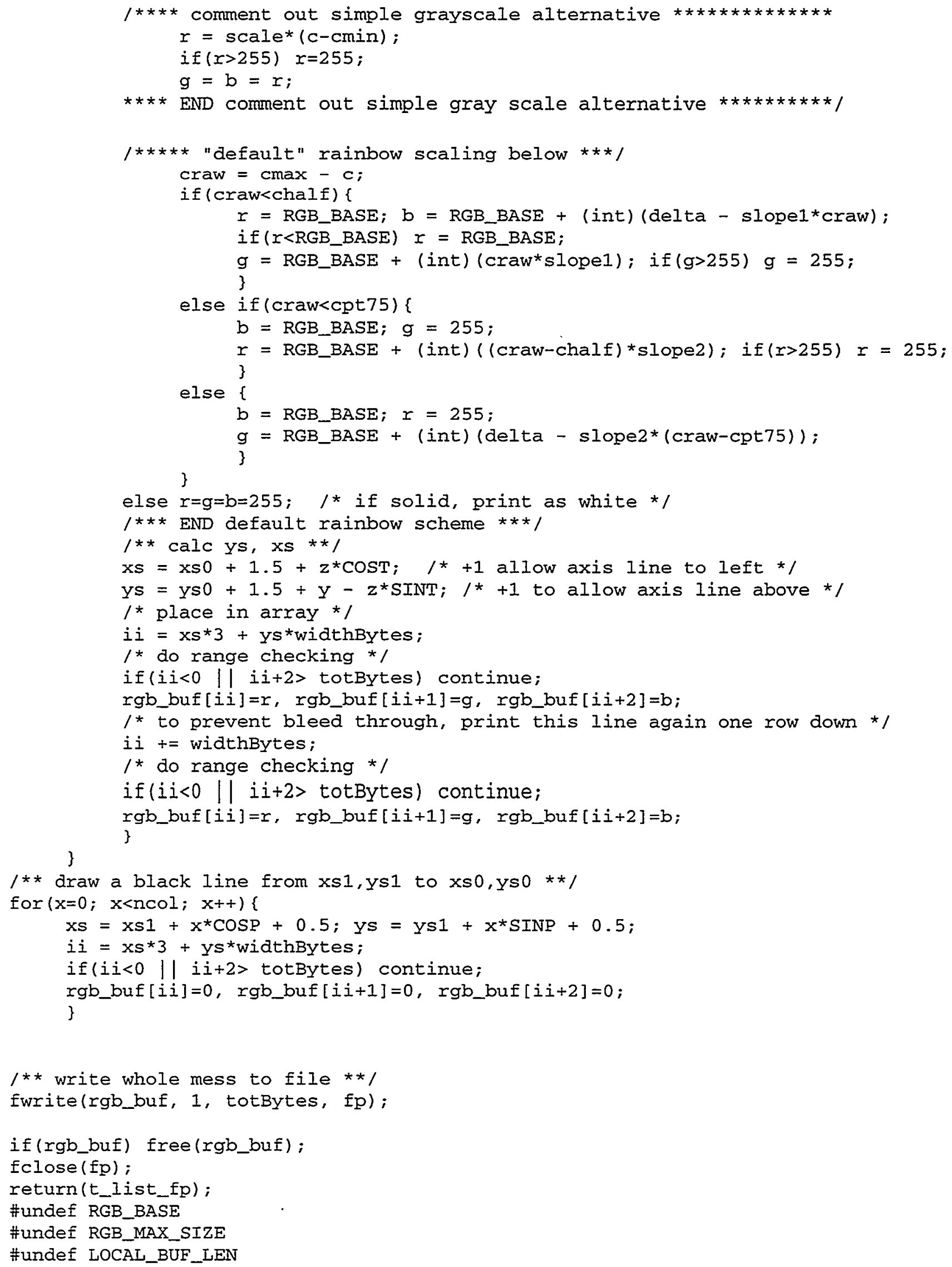


\} $\quad$ * END Save3DPpm() */

$1 *$ Modify SaveUPpm() to save an HPGL vector file, of the 4 layers...* $/$

$1 *$ navg tells how many points in a line to include in an average, */

1* nskip tells how many points to skip between plots/averages, */

/* maxVectFrac gives the fraction of the largest dimension (e.g. ncol */

1 * or nlay) to make the largest vector... */

$1 *$ 2-21-98 modify to (1) allow "log" (really fourth root) scaling), and */

$I^{*}$ (2) put the average of two cells in between points averaged... */

1 * Change to pass nxavg, nyavg and NOT use nskip, ADD useLog, useColor * $/$

1 * 7-15-97 modify to save separate files for each $z z[n]$.. HPGL just doesn't * $/$

$1 *$ handle the resizing well...* $/$

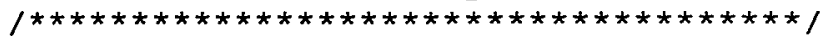

int SaveVect/struct veloc_t $* * *$ const uu, char $* * *$ solid,

int nlay, int nrow, int ncol, int $z z[]$, int $n z$, int step,

int nxavg, int nyavg, int useLog, int usecolor, float maxVectFrac)

\{

\#define LOCAL_BUF_LEN 256

static int filenum $=0$, firsttime $=1$;

static FILE * fp;

int $i, i i, x, y, z, n$, xend, yend; $/ * 2-21-98$ xend, yend *

$1 *$ All coords below are in SCREEN coords, and the vertical (y) screen coords *

/* have opposite sense as $y$ index into rows... */

/* $\mathrm{x} 0$ and $\mathrm{y} 0$ are offsets from page edge, for each frame; */

float $x 0, y 0$, yinc;

$1 *$ yinc is the increment in $y 0$ from frame to frame... */

float $x c, y c, d x, d y, x 1, y 1, x 2, y 2$;

$I^{*} \mathrm{xc}, \mathrm{yc}$ is the center point of each arrow; the arrow shafts will be */

I* symmetrically disposed about xc,yc. */

double maxVectScreenUnits, uLengthscale, colorScale;

box_t clipBox;

/* 2-21-98 add these: *I

int $\mathrm{xx}, \mathrm{yy} ; \quad /$ for incrementing in an average block * $/$

float $x A v g I n c$, YAvgInc; $/$ * these are offsets form $x, y$ for average of points * double uLogLengthScale;

int doAvg; $1 *$ set if EITHER nxavg OR nyavg==0.. */

$1 *$ pointers to save time: */

struct veloc_t $* *$ uuz;

char ** solidz;

int nopen; $I^{*}$ in averaging, the number of open (non-solid) points in block */

int pagewidth, pageHeight, color;

unsigned char $r, g, b$;

double u, u2, u2max $=-1.0 e 200$, umax, ux, uy, mLog; $/$ * 2-21-98 mLog */

struct tm *ptm;

static int day $=0$, hour $=0 ; /$, because localtime destroys single internal tm *

time_t 1t;

char buf[LOCAL_BUF_LEN]; /* for filenames */

$1 *$ if we want to write directly to printer in future, should */

$1 *$ add $1033 \% 0 \mathrm{~B} \backslash \mathrm{n}$ at beginning of file, and $1033 \%$ at end * $/$

static char *initCode="IN;RO90;IP;IW; SC\%d, 8d, 8d, 8d;FT1;PA; SP1;PW0.2; In",

*endCode="PU;",

*beforeSolids="PU; PU ; PU ; PU ; PU ; PU ; PU; PU ; PU ; PU ; PWO . 5 ; In" ;

$/ *$ beforesolids allows us to find and remove solids block, and * $/$

$1 *$ overlay with bitmap if we want... */ 


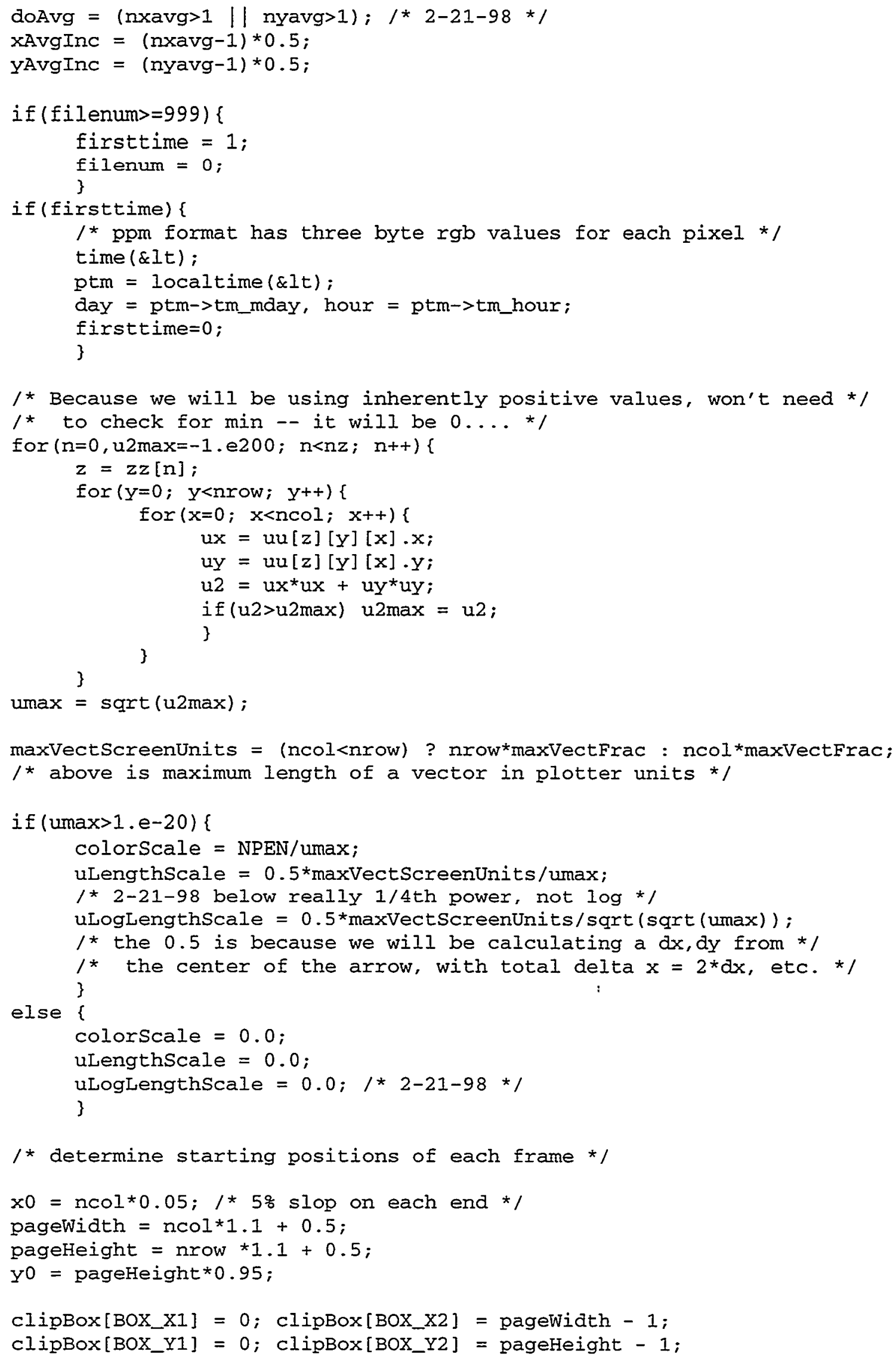


/* first loop over all the vectors, so we can print solids separately, and */ 1 * remove them later if we want to overlay a bit map */

for $(n=0 ; n<n z ; n++)\{$

$\mathrm{z}=\mathrm{zz}[\mathrm{n}]$;

uuz $=$ uu $[z] ;$ solidz $=$ solid $[z] ; / * 2-21-98 * /$

$1 *$ make the file name; avoid lots of unclosed files if */

$1 *$ the umax is too small... * $/$

/* Remember localtime structure is not "re-entrant"... */

buf [LOCAL_BUF_LEN-I] $=0$;

sprintf(buf, "V\&02d\%02d\%03d.803d", day, hour, filenum, z);

fp = fopen (buf, " $w ") ; / *$ open as ascii */

if(!fp) return (0); $/$ * was return(0) */

$1^{*}$ write HPGL header to define page size 7-15-97 move here *

fprintf(fp, initCode, 0 , pagewidth, 0 , pageHeight);

I* draw a box around the current $z$ slice 7-15-97 $+/-0.5$ added */

DrawBox $(x 0+0.5, x 0+n c o l-0.5, y 0$-nxow $+0.5, y 0-0.5$, clipBox, $1 p, 1)$;

for $(y=0 ; y<$ nrow; $y+=$ nyavg) \{

for $(\mathrm{x}=0 ; \mathrm{x}<\mathrm{ncol} ; \mathrm{x}+=\mathrm{nxavg})\{$

if (doAvg) $\{\quad 1 * 2-21-98$ averaging block */

for ( $u x=u y=0.0, y y=y, y e n d=y+n y a v g$, nopen $=0 ; y y<y$ end; $y y++)\{$

for $(\mathrm{xx}=\mathrm{x}$, xend $=\mathrm{x}+\mathrm{nxavg} ; \mathrm{xx}<\mathrm{xend} ; \mathrm{xx}++)\{$

if (solidz [yy] $[x x])$ continue;

$u x+=u u z[y y][x x] \cdot x$;

$\mathrm{uy}+=\mathrm{uuz}[\mathrm{Yy}][\mathrm{xx}] \cdot \mathrm{y}$;

nopen++;

\}

\}

if (nopen) $\{\mathrm{ux} /=$ nopen; uy/=nopen; $\}$

else continue; /* don't plot a pure solid vector */

$\mathrm{xc}=\mathrm{x} 0+\mathrm{x}+\mathrm{xAvgInc}+0.5$

$y c=y^{0}-y-y$ AvgInc $-0.5 ;$

else \{

if(solidz $[y][x])$ continue;

$u x=u u z[y][x] \cdot x$;

$u y=u u z[y][x] \cdot y ;$

$x c=x 0+x+0.5 ; y c=y 0-y-0.5 ;$

\}

$\mathrm{u}=\operatorname{sqrt}\left(\mathrm{ux}^{\star} \mathrm{ux}+\mathrm{uy}^{\star} \mathrm{uy}\right)$;

$1 *$ calc endpoints of the line to plot */

if (usecolor) color $=$ colorScale*u;

else color $=0 ; \quad$ * 2-21-98 add if for monotone *

if (uselog $==0$ ) \{

$d x=$ uLengthScale*ux; $d y=$ uLengthScale*uy;

\}

else if $(u>1.0 e-50)\{/ * \log$ scale */

mLog $=$ uLogLengthScale*sqrt $(\operatorname{sqrt}(u)) / u$;

$d x=u x{ }^{*} \operatorname{mLog} ; d y=u y{ }^{*} \mathrm{mLog}$;

\}

else continue; $/ *$ don't print a zero vector */

$\mathrm{x} 1=\mathrm{xc}-\mathrm{dx} ; \mathrm{x} 2=\mathrm{xc}+d \mathrm{x} ; \mathrm{y} 1=\mathrm{yc}-\mathrm{dy} ; \mathrm{y} 2=\mathrm{yc}+\mathrm{dy} ;$

DrawArrow $\left(x 1, y 1, x 2, y 2,0.3,0.7\right.$, color, $\mathrm{fp}_{\mathrm{p}}$ clipBox); 


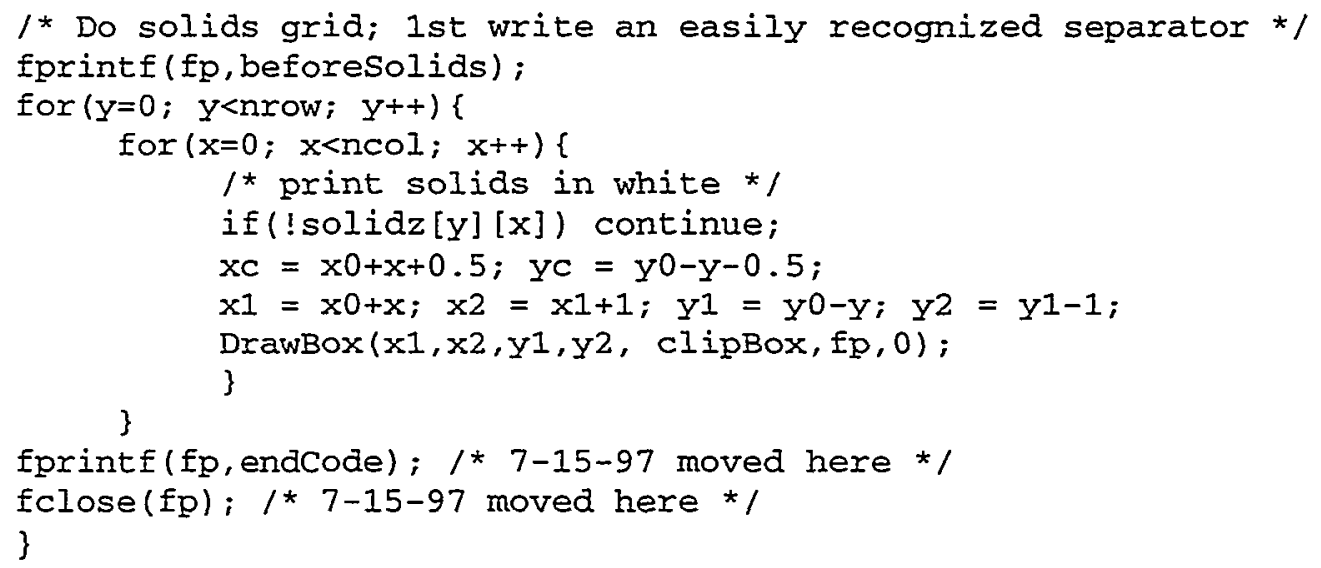




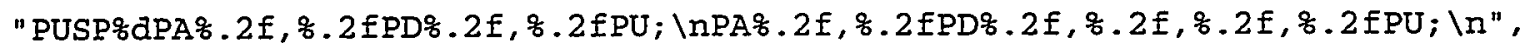
penColor [color] $, \mathrm{xx} 1, \mathrm{yy} 1, \mathrm{xx} 2, \mathrm{yy} 2, \mathrm{xx} 4, \mathrm{yy} 4, \mathrm{xx} 2, \mathrm{yy} 2, \mathrm{xx} 5, \mathrm{yy} 5)$; \}

1 * kludge to make yellow more visible in word */

if (color $==$ iYellow \&\& iYellow $<N P E N-1)$ \{

fprintf(hpgl_fp, "LT2,0.1,1; (n");

if(status1 \&\& status2 \&\& status 3$)\{$

fprintf(hpgl_fp,

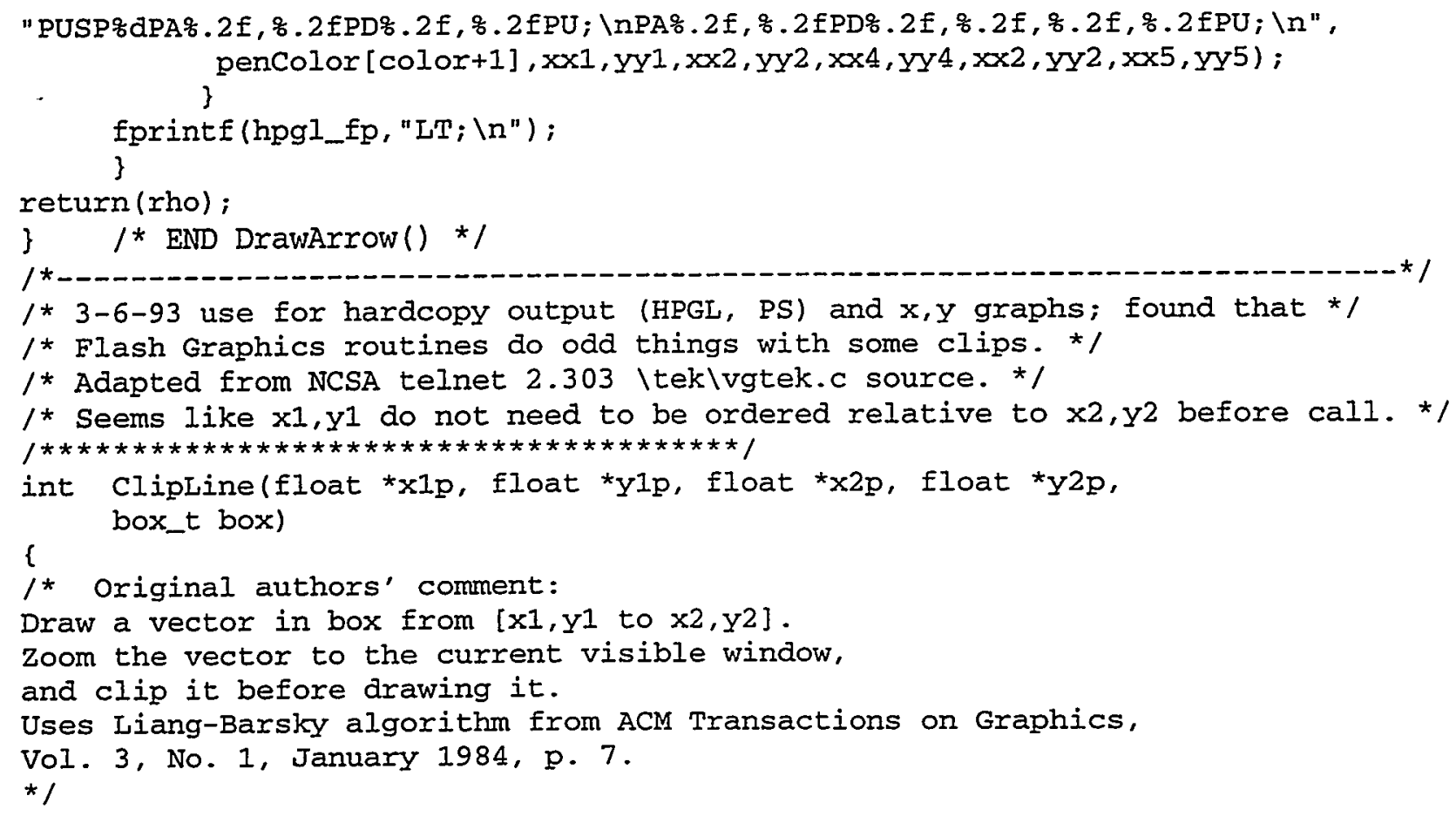




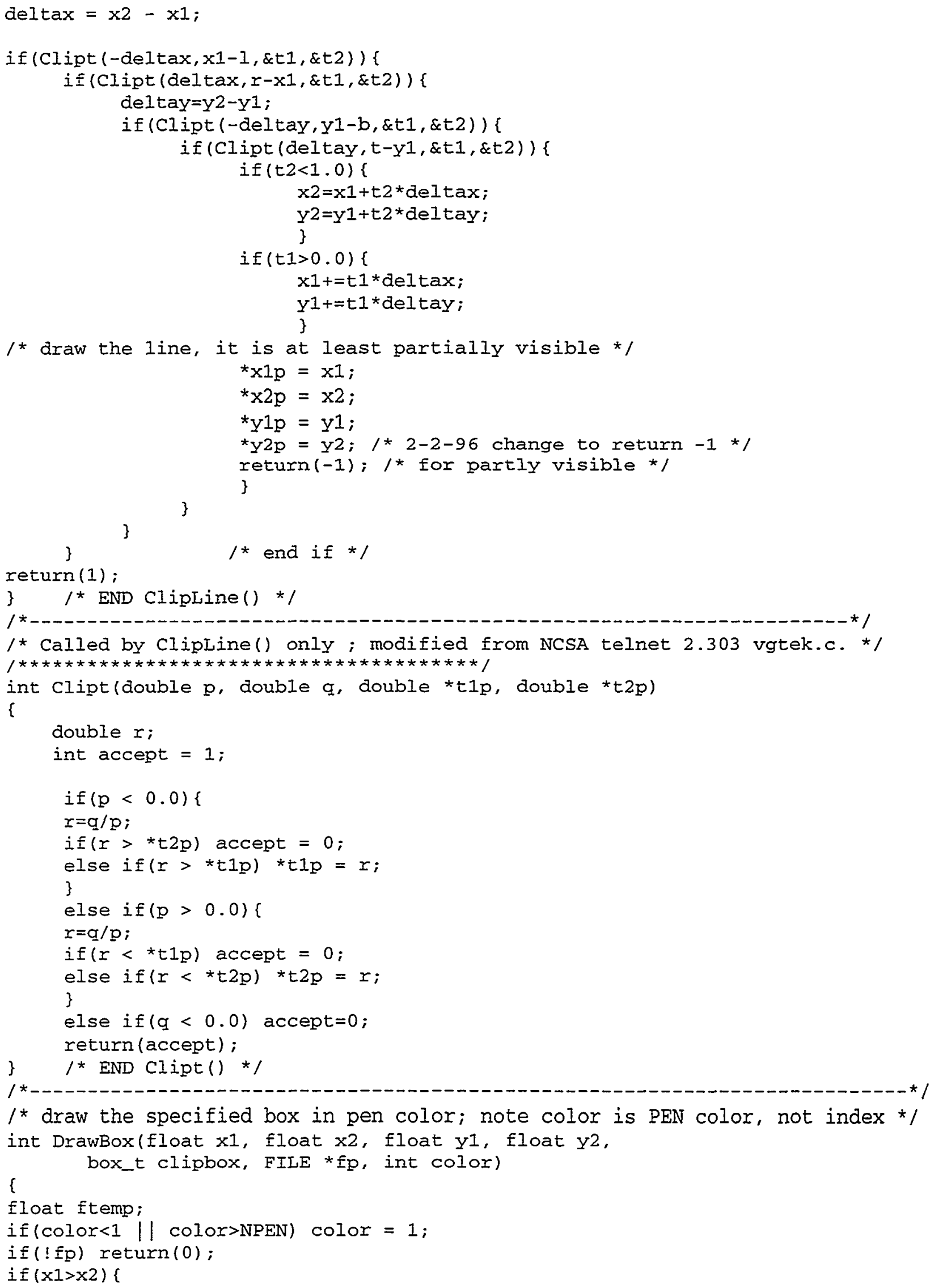




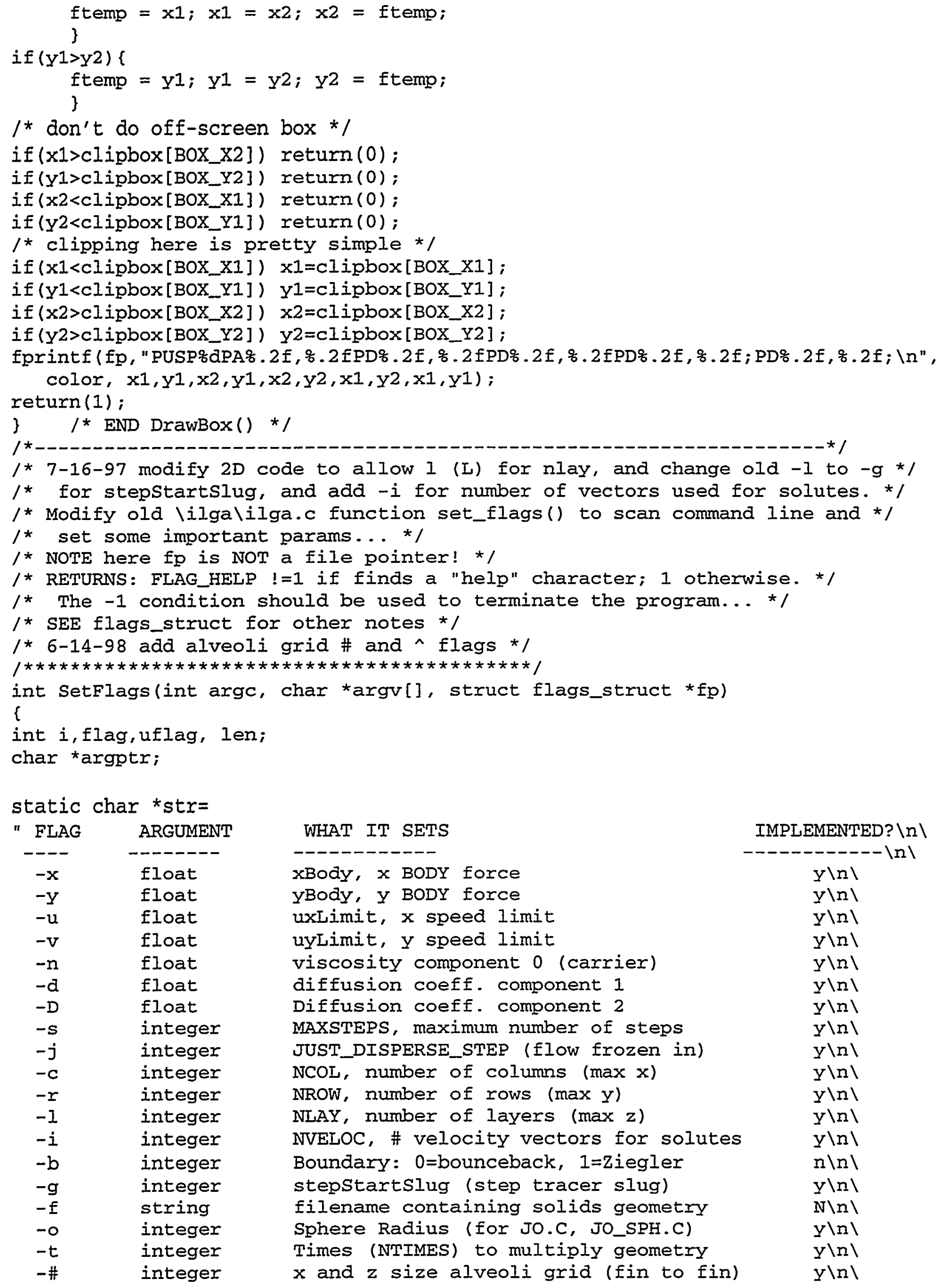




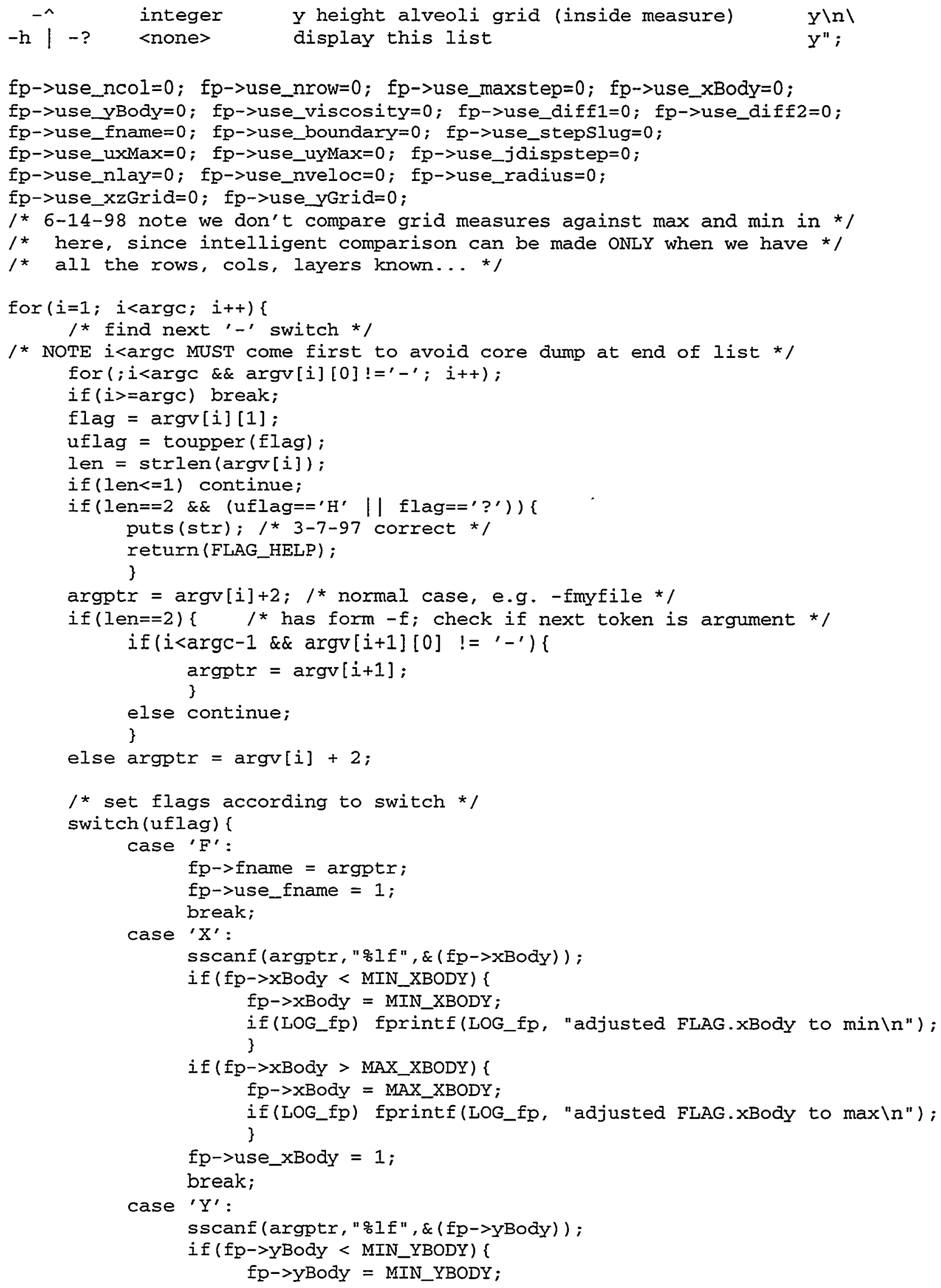




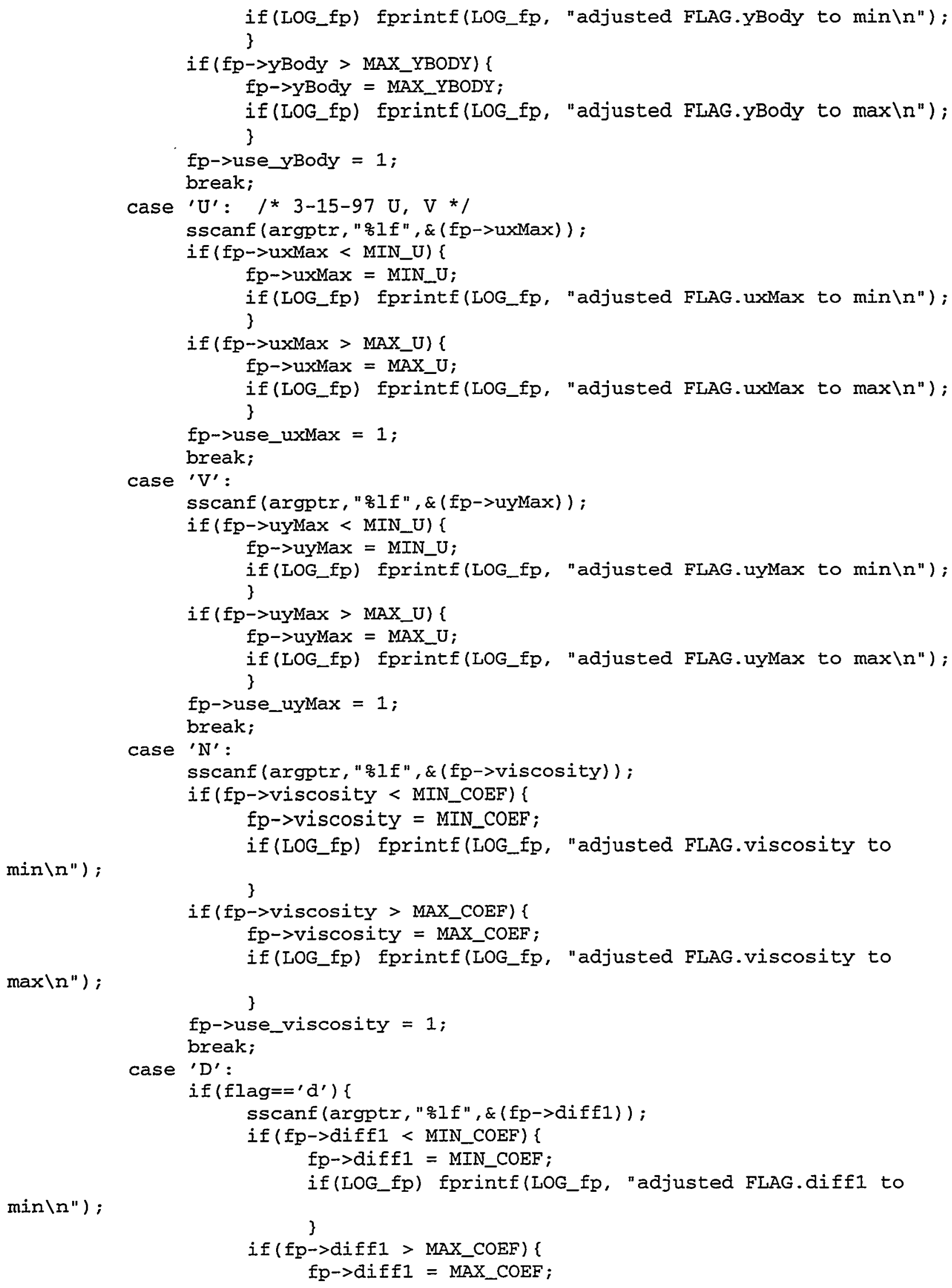


$\left.\max \backslash \mathrm{n}^{\prime \prime}\right)$;

if(LOG_fp) fprintf(LOG_fp, "adjusted FLAG.diffl to

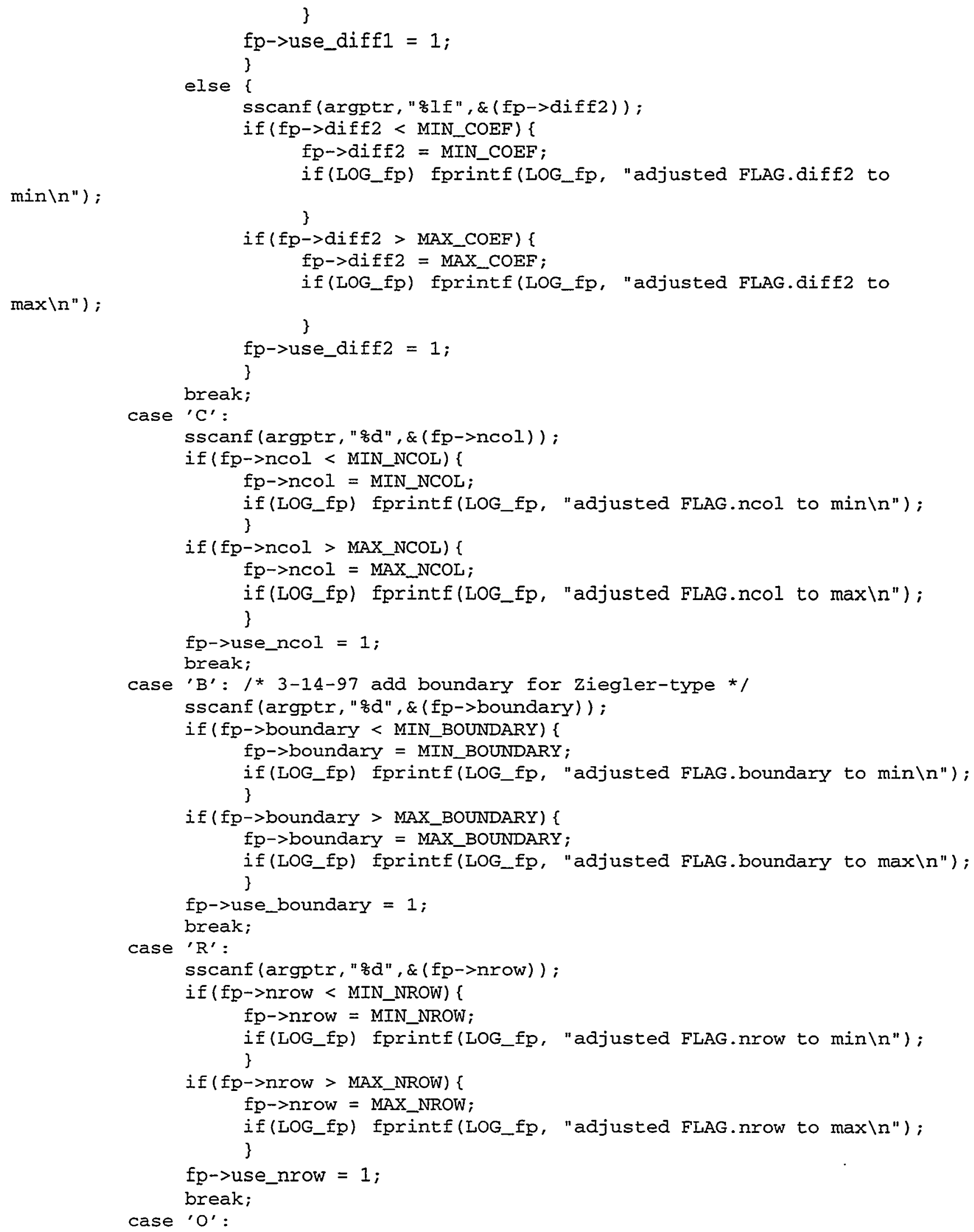




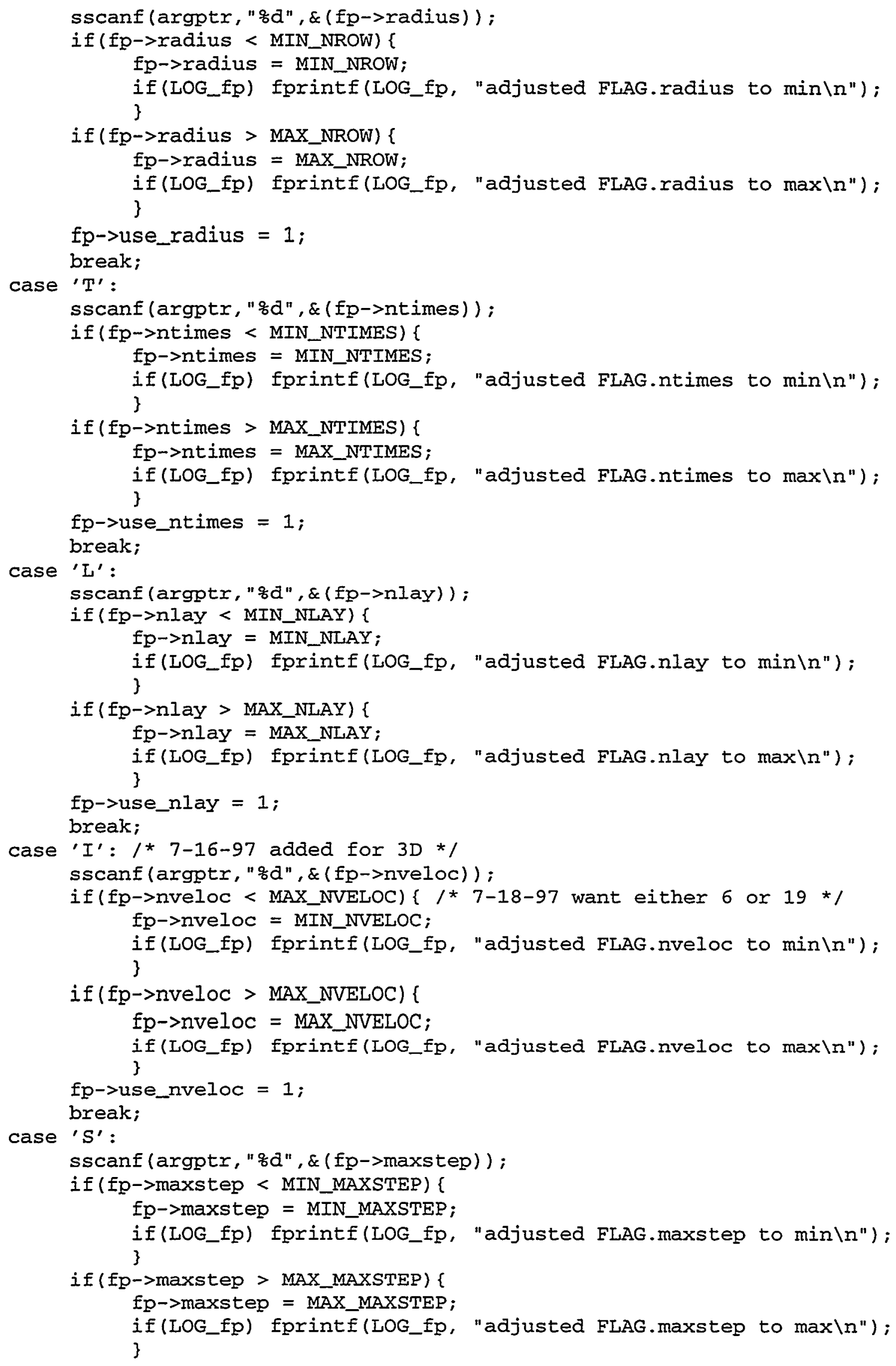




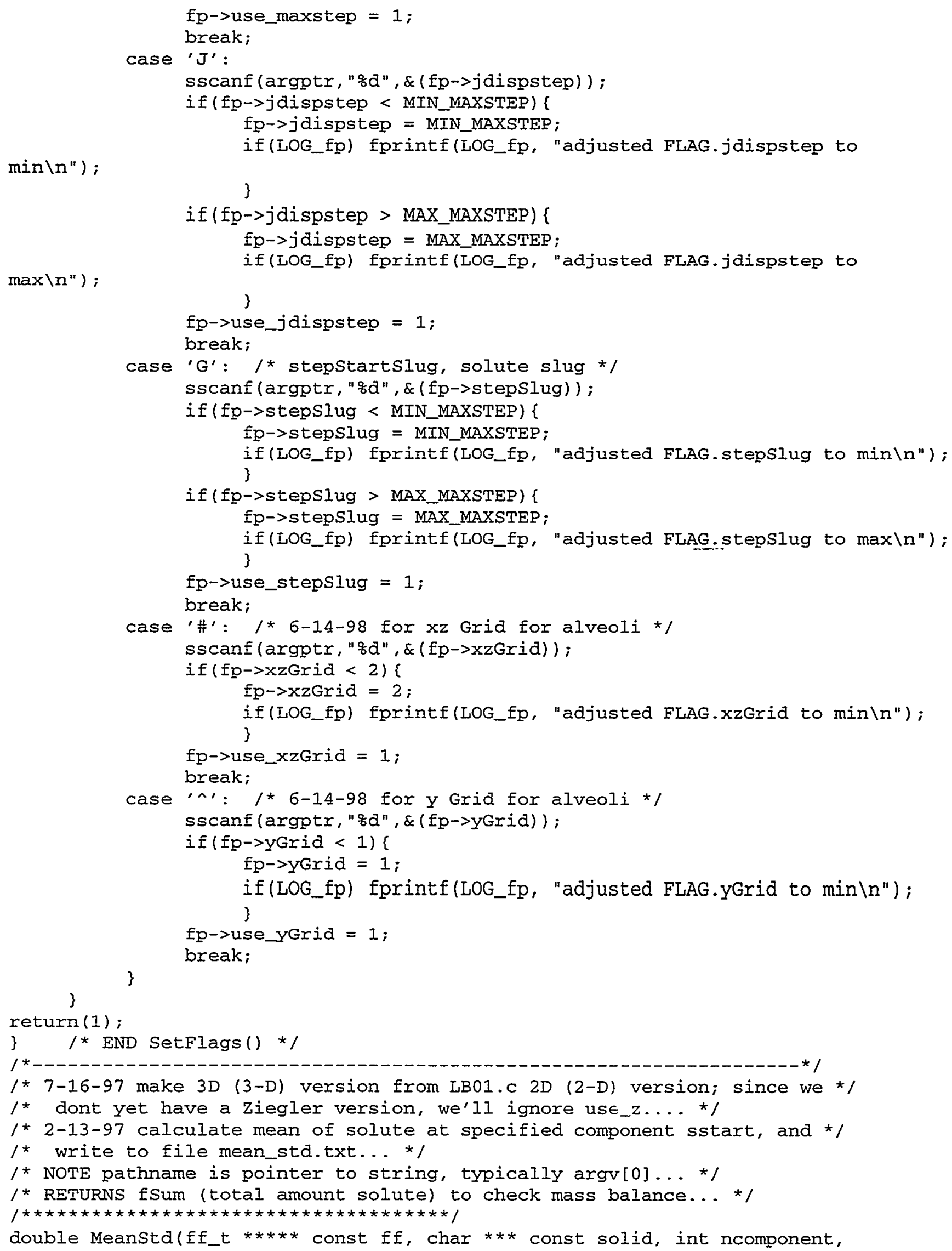


int nveloc[], int nlay, int nrow, int ncol, int sstart, int step, char *pathname, int use_z)

\{

int $x, y, z, i$;

static int firstime $=0$;

FILE *fp;

double fx2Sum, fxSum, f, fSum, fy2Sum, fySum, xtemp, ytemp, $x x, y y$;

double xMean, xVar, xstd, yMean, yVar, yStd;

/ 7-16-97 add: */

double zz, fz2Sum, fzSum, ztemp, zMean, zVar, zStd;

/* currently assume solids will have valid ff values */

struct tm *ptm; $\quad / * 2-22-97$ convert to localtime() in time.h *

time_t 1t;

char *str;

int sc; $/$ * solidcode */

$f_{p}=$ fopen ("mean_std.txt", "a");

if $(! f p)$ return $(-1.0)$;

if (firstTime $=0$ ) \{

1* 3-17-97 use use_ziegler instead of flags... */

if (use_z) str = "Ziegler";

else str = "BounceBack";

time (\&lt);

ptm $=$ localtime $(\& l t)$;

fprintf $\left(\mathrm{fp}, "========8 \mathrm{~s} \quad 802 \mathrm{~d} / 802 \mathrm{~d} / 804 \mathrm{~d}\right.$ \% $02 \mathrm{~d}: 802 \mathrm{~d}: \frac{8}{8} 02 \mathrm{~d}========1 \mathrm{n} "$, pathname, ptm->tm_mon +1 , ptm->tm_mday, ptm $>t m \_y e a r+1900$, ptm $\rightarrow$ tm_hour, ptm $>$ tm_min, ptm->tm_sec);

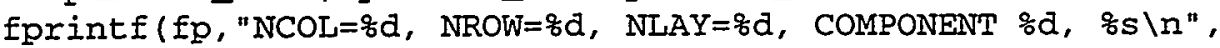
ncol, nrow, nlay, sstart, str);

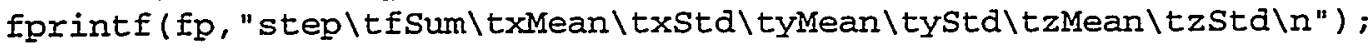

firstTime $=1$;

\}

Ex2Sum $=0.0$, fxSum=0.0, fSum=0.0, fy $2 \mathrm{Sum}=0.0$, fySum $=0.0 ;$

fz2Sum $=0.0$, fzSum $=0.0 ; / * 7-16-97 * /$

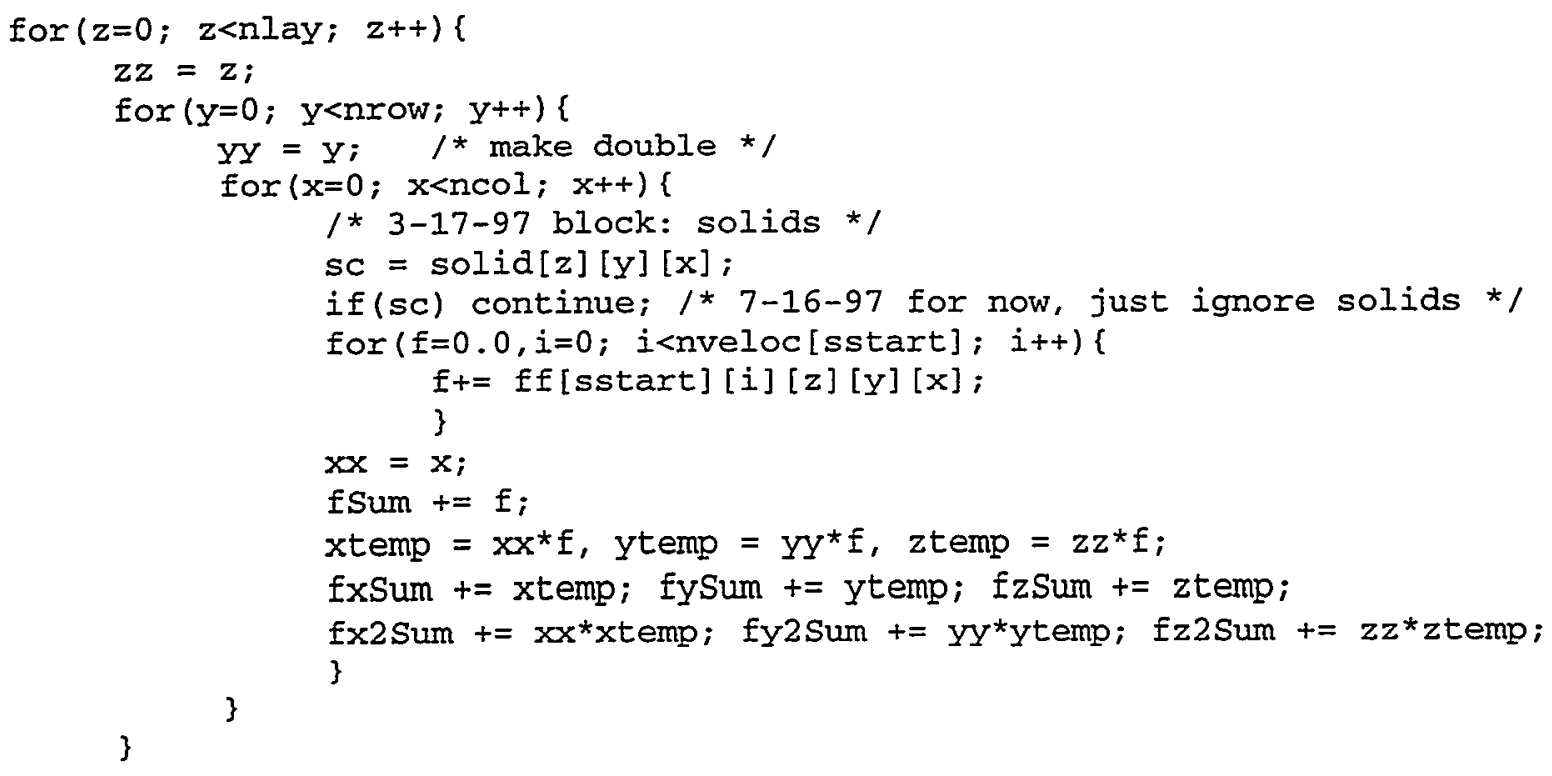




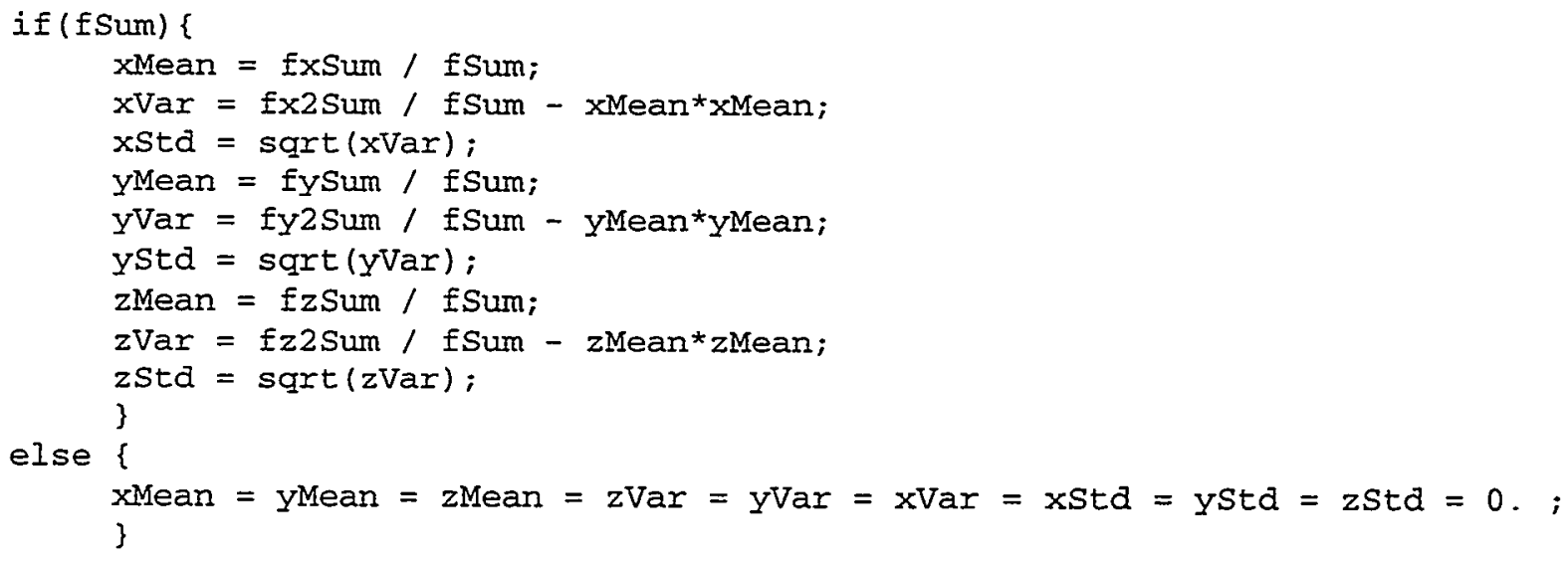




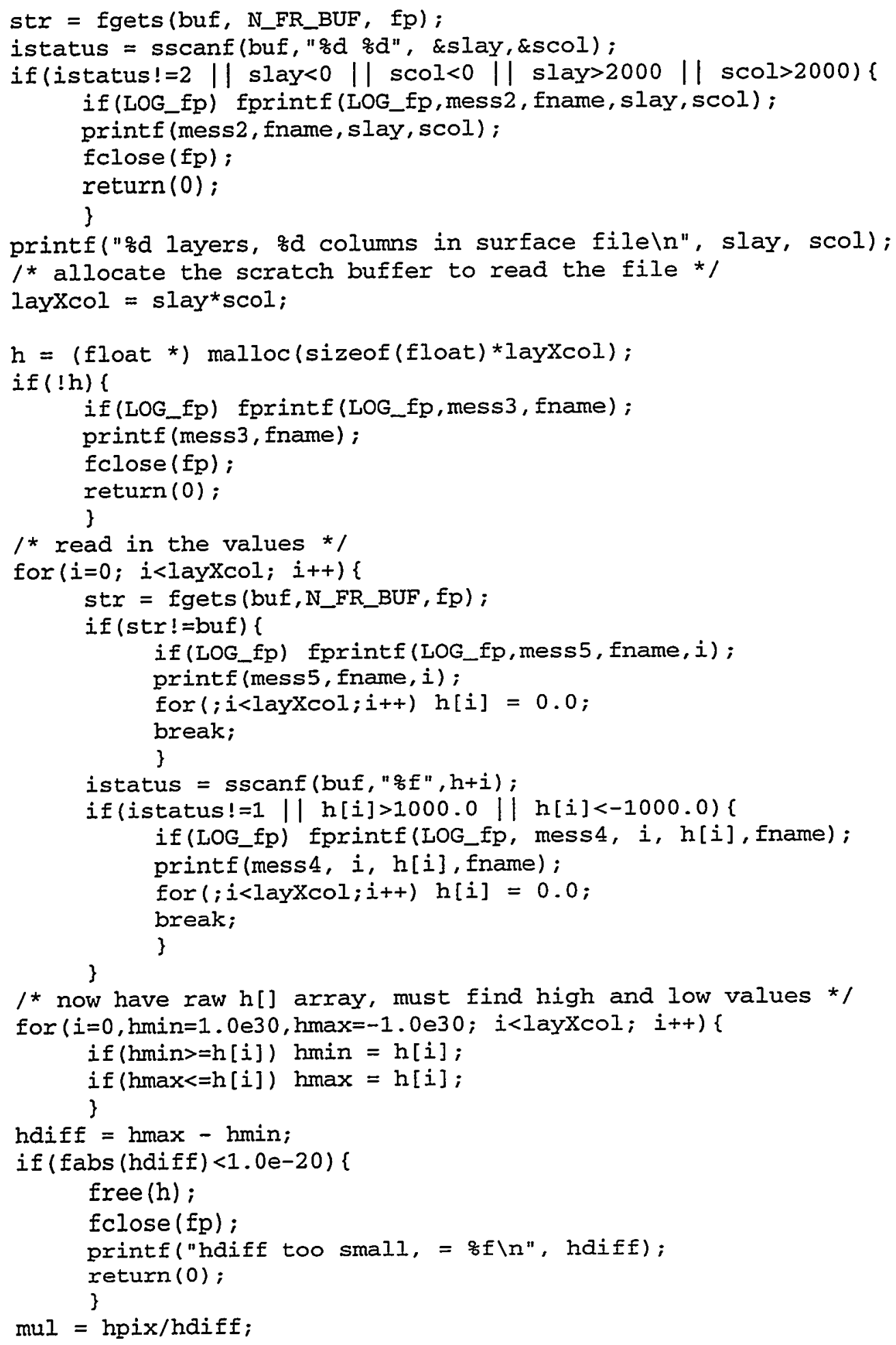




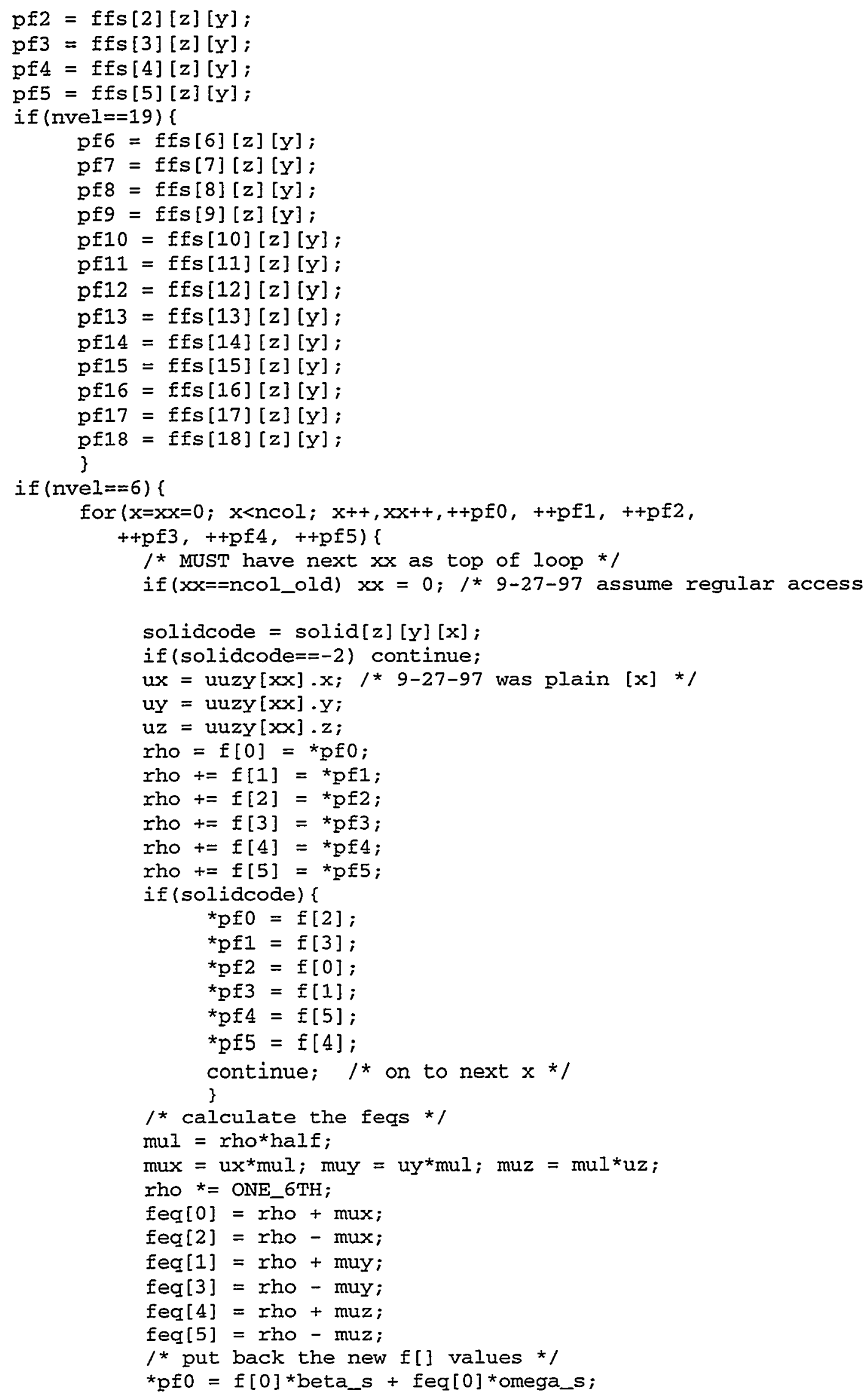




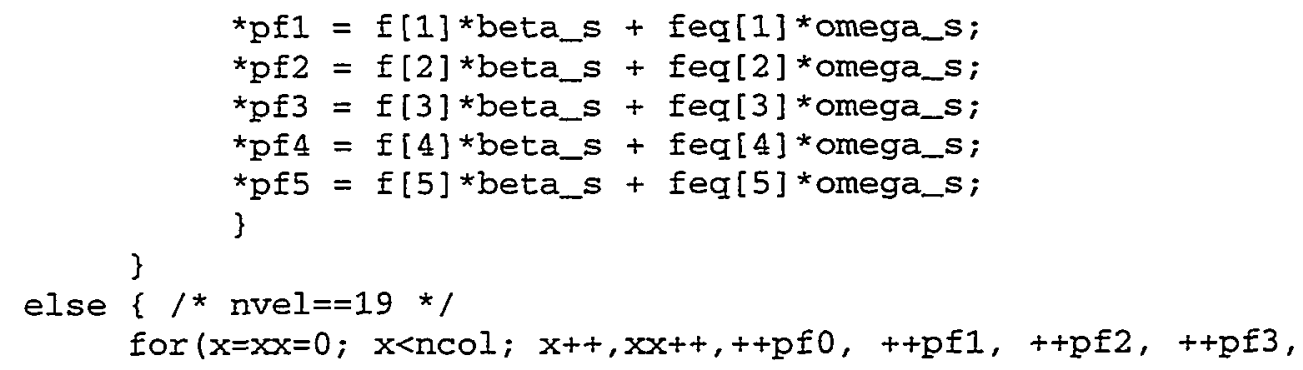

++ pf4, -

$++p f 5,++p f 6,++p f 7,++p f 8,++p f 9,++p f 10,++p f 11,++p f 12$,

$++p f 13,++p f 14,++p f 15,++p f 16,++p f 17,++p f 18)\{$

1 * MUST have next $\mathrm{xx}$ as top of loop */

if $\left(x x==n c o l \_o l d\right) \quad x x=0 ; / * 9-27-97$ assume regular access

solidcode $=$ solid $[z][y][x]$;

if (solidcode==-2) continue;

$\mathrm{ux}=\mathrm{uuzy}[\mathrm{xx}] . \mathrm{x} ; /{ }^{*} 9-27-97$ was plain $[\mathrm{x}]$ */

uy $=$ uuzy $[x x] \cdot y ;$

$\mathrm{uz}=\mathrm{uuzy}[\mathrm{xx}] \cdot \mathrm{z}$;

1 * 2-27-98 rearrange order for read-buffer bug? */

rho $=f[0]={ }^{*} \mathrm{p} f 0$

rho $t=f[4]={ }^{*} p f 4$;

rho $t=f[8]={ }^{*}$ pf 8 ;

rho $t=f[12]=* p f 12$;

rho $+=f[16]=* p f 16$;

rho $t=f[1]={ }^{*} p f 1$;

rho $+=f[2]={ }^{*} p f 2$;

rho $t=f[3]={ }^{* p f 3}$;

rho $+=f[5]={ }^{*} p f 5$;

rho $t=f[6]=* p f 6$;

rho $t=f[7]=*$ pf7;

rho $+=f[9]=* p f 9$;

rho $+=f[10]=*$ pf10;

rho $+=f[11]={ }^{*}$ pf11;

rho $+=f[13]={ }^{*}$ p f13;

rho $t=f[14]={ }^{*}$ pf14;

rho $+=f[15]={ }^{*} p f 15$;

xho $+=f[17]={ }^{*} \operatorname{pf17}$;

rho $+=f[18]={ }^{*} p f 18$;

if (solidcode) \{

/* BounceBack (f); * /

/ * 10-16-97 stop; inline BounceBack() */

$1 *$ in xy plane * 1

$* \mathrm{pf5}=\mathrm{f}[1] ; * \mathrm{pf} 1=\mathrm{f}[5]$;

$* p f 7=f[3] ; *$ pf3 $= \pm[7] ;$

${ }^{*} \mathrm{pf6}=f[2] ;{ }^{*} \mathrm{pf} 2=\mathrm{f}[6] ;$

*pf8 $=f[4] ; *$ pf4 $=f[8]$;

$I^{*} z$ up-and down vectors *I

${ }^{*}$ pf18 $=f[9] ;{ }^{*}$ pf9 $=\mathrm{f}[18]$;

* off-xy-plane vectors */

${ }^{\star} \mathrm{pfl} 16=\mathrm{f}[10] ;{ }^{*} \mathrm{pf10}=\mathrm{f}[16] ;$

${ }^{*} \operatorname{pf12}=f[14] ;{ }^{*} \operatorname{pf14}=f[12] ;$

${ }^{*}$ pf17 $=f[11] ; *$ pf11 $=f[17] ;$

$*$ pf15 $=f[13] ; *$ pf13 $=f[15] ;$

continue;

\} 


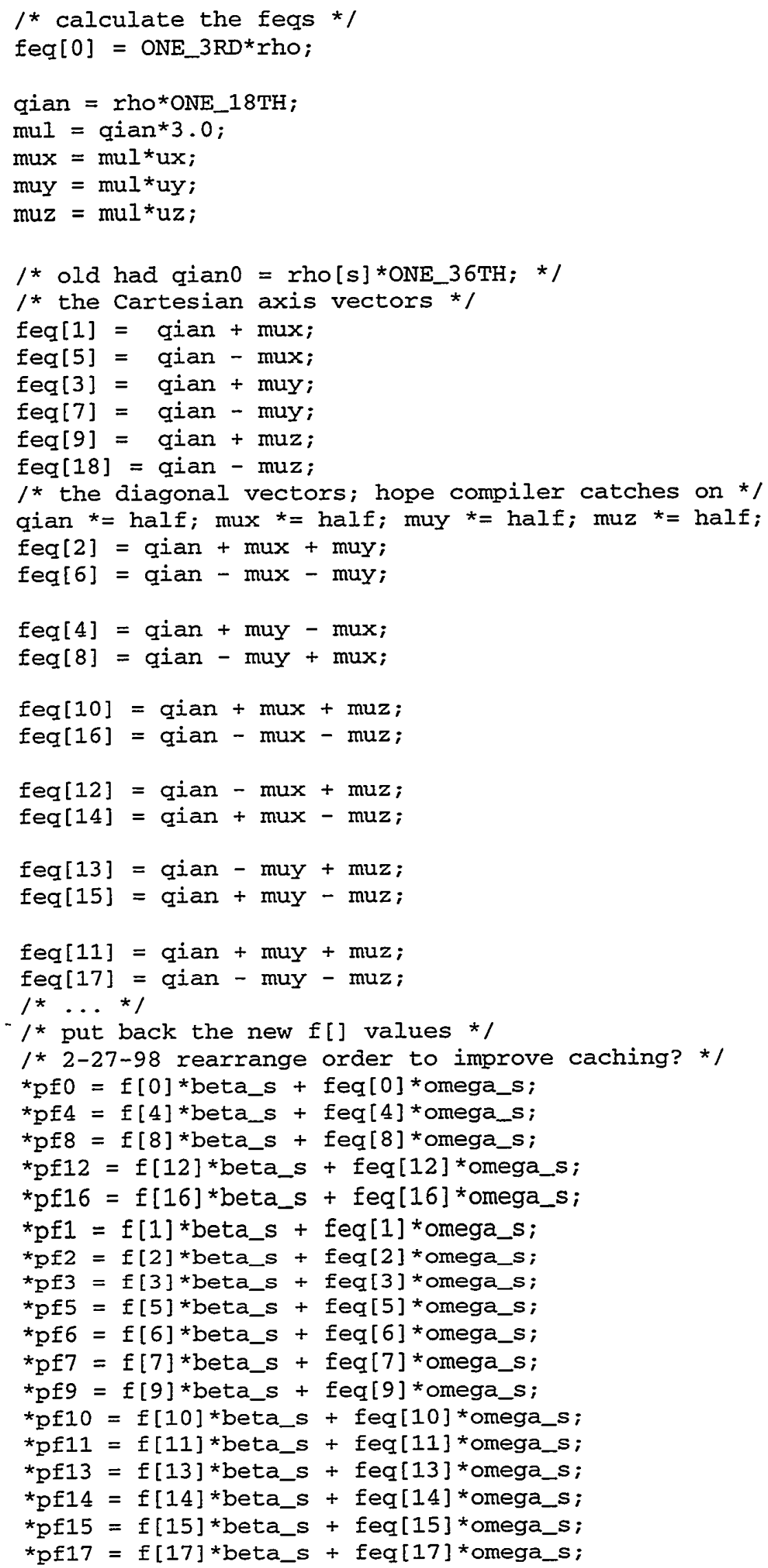




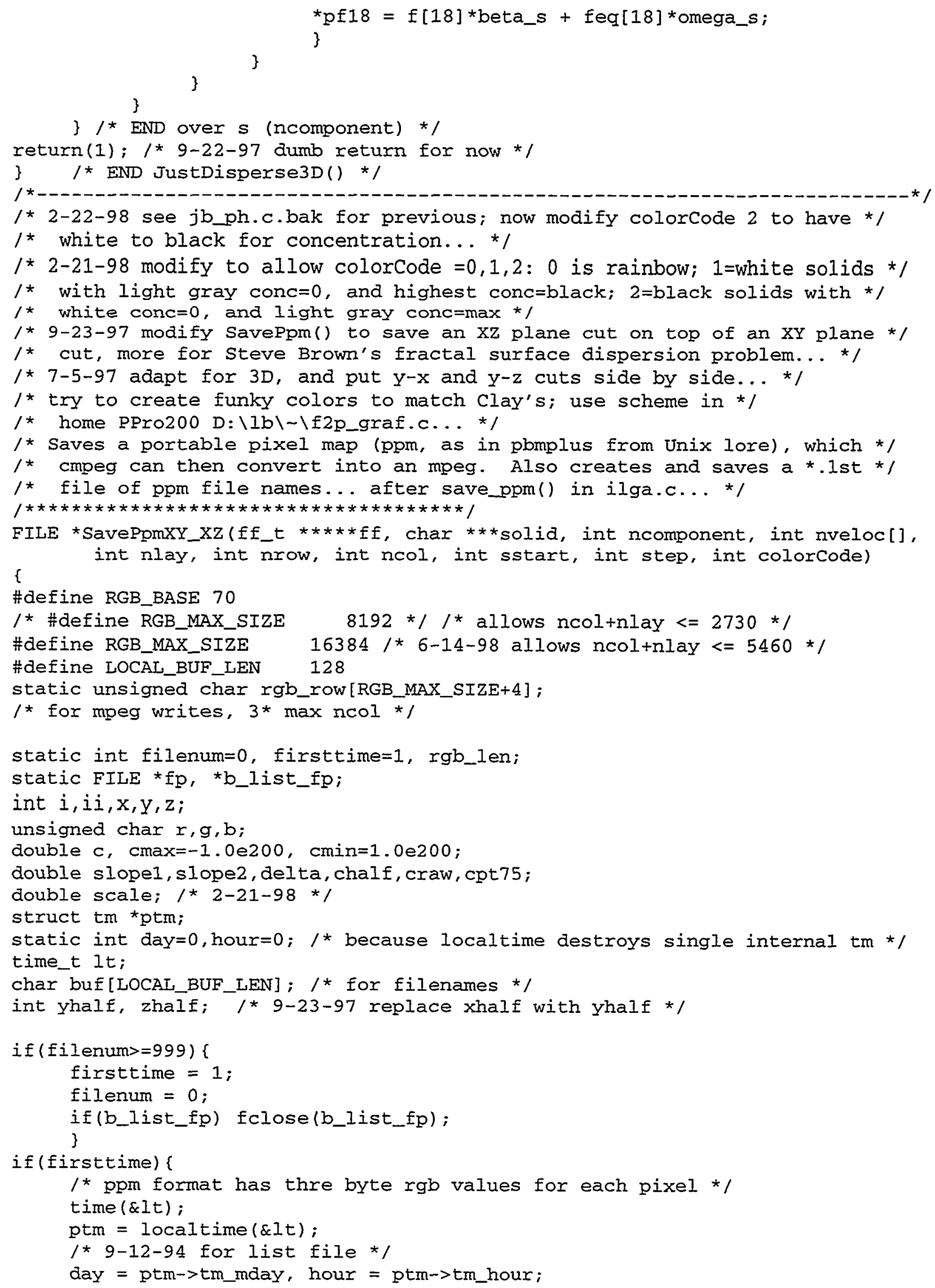




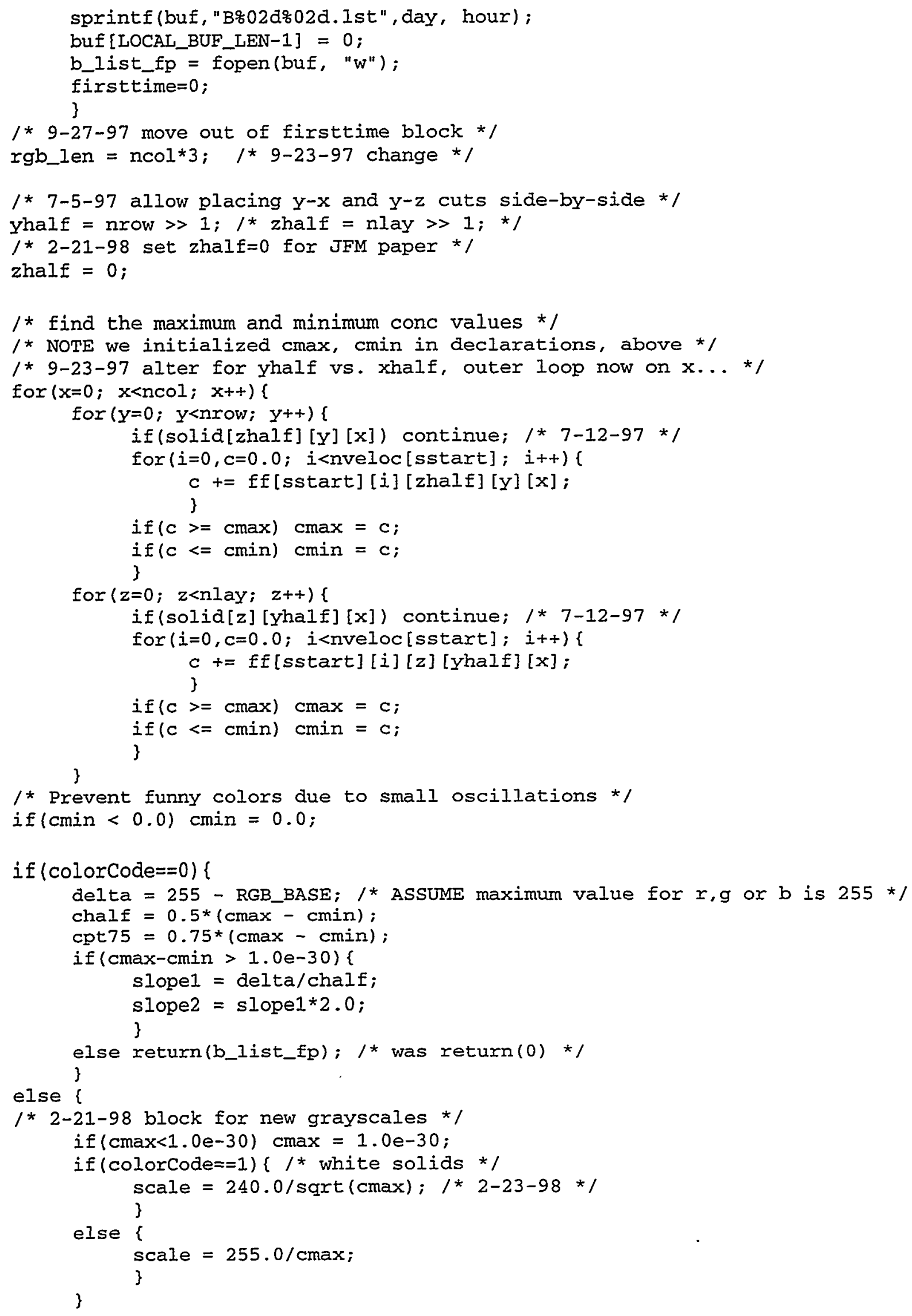




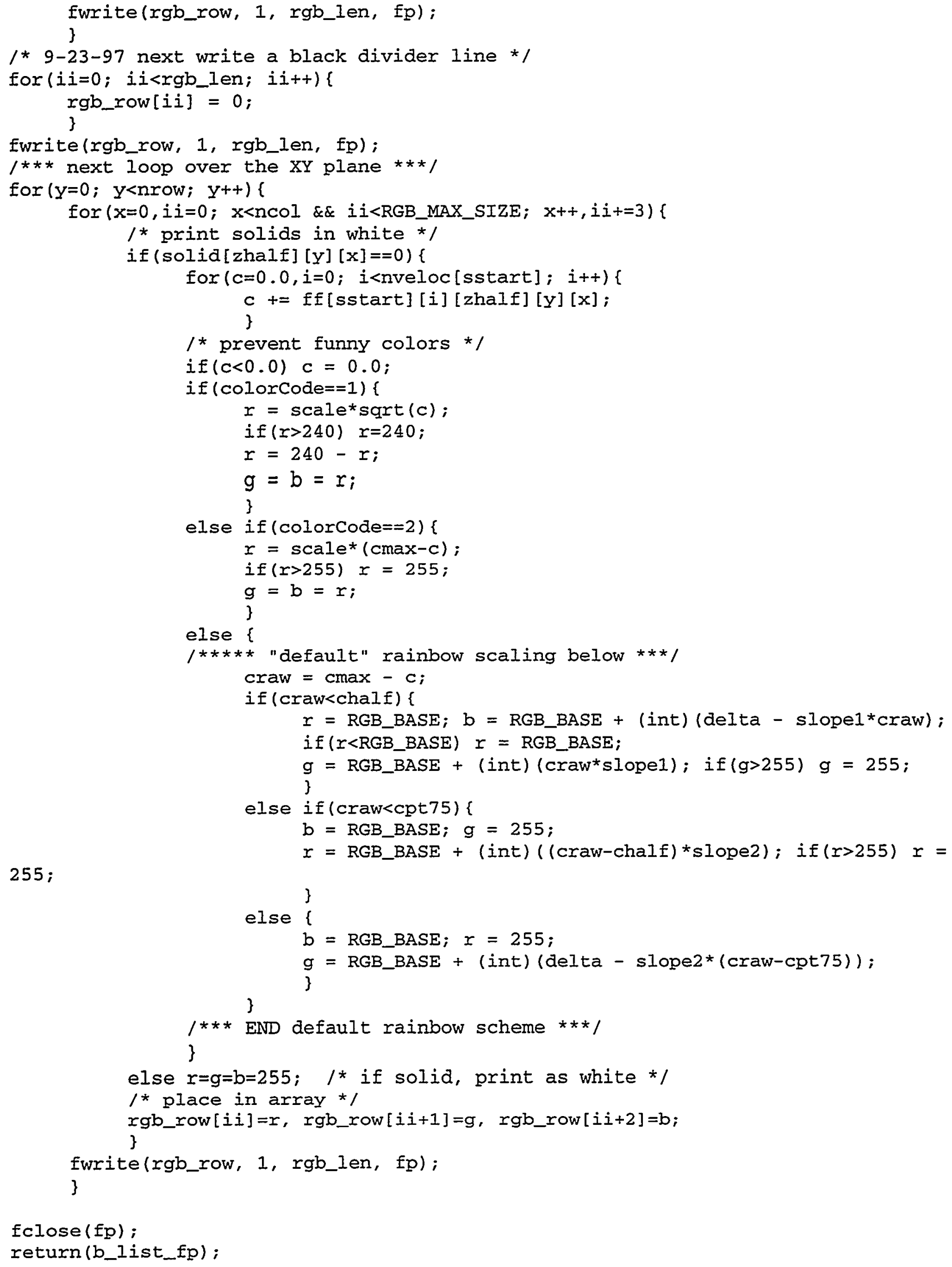


\#undef RGB_BASE

\#undef RGB_MAX_SIZE

\#undef LOCAL_BUF_LEN

\} $\quad / *$ END SavePpmXY_XZ () */

1*-1.--

/* 9-23-97 modify Ib01\lb04.c function ReadBmp4Slug() to extrude */

$1 *$ slug along $y$, and since we may eventually use in situation with */

1 * ff[0][i] dellocated, just distribute the concentrations equally */

$1 *$ (scaled to e.g. $1 / 3$ or $1 / 18$ or $1 / 36$ for $\mathrm{D} 3019$ ) over vectors... */

/* 6-2-97 try reading in bmp files to use as input for 1b01.c. */

1 * This func reads slug file only, interprets any color darker */

/* than brightest color as solute at sstart... */

/* NOTE unlike Setslug(), takes a single rho, not conclis... */

1* Modify from bmp.c, created under home GW2K PPro200 D: $11 \mathrm{~b}$ for * 1

$1 *$ easy debugging. * $/$

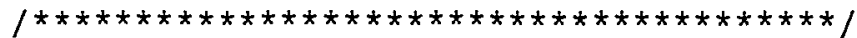

int ReadBmp4Slug (ff_t $* * * * *$ const ff, char $* * *$ const solid, float rho, int nvel, int nlay, int nrow, int ncol, int sstart,

int $x$, int $z$, char *ilename)

\{

int $i, j, k, m, n$, istatus, kend, zoffs, $x x, y y, z z$;

FILE * $\mathrm{fp}_{\text {; }}$

char buf[32];

double ftemp, rhoo;

ff_t $f\left[D E F \_N V E L O C\right], f 6$;

int fSize, dumn, width, height, compression, sizeImage, colorsUsed, colors Imp;

short planes, bitcount, bitsPerpix;

unsigned char rgb[4];

int jmax, jmin, max, min, sum, rowbytes, color;

unsigned char *rowBuf;

/ open the passed filename */

$f p=$ fopen (filename, " $r b ")$;

if (! fp) \{

printe("can't open os $\backslash n$ ", filename);

return (0):

\}

istatus $=\operatorname{fread}(\mathrm{buf}, 1,2, \mathrm{fp}) ;$

if(istatus $<2$ || buf[0]!='B' || buf[1]!='M') \{

puts ("Not a valid windows bitmap file");

fclose ( $\mathrm{fp}$ );

return $(0)$;

\}

puts ("got here! saw BM!");

istatus $=$ fread $(\& f S i z e, 4,1, f p) ; \quad / *$ size of this ? file ? */

printf("this file is od bytes long $\backslash n$ ", fSize);

istatus $=$ fread (\&dummy, $4,1, \mathrm{fp}) ; 1$ * reserved1, reserved 2 *

istatus $=$ fread $(\& d u m m, 4,1, \mathrm{fp}) ; \quad /$ offsetBytes * $/$

printf("Offset bytes to data: od $\backslash n^{\prime}$, dummy);

/* read BitmapInfoHeader */

istatus $=$ fread $(\& d u m m y, 4,1, \mathrm{fp}) ; \quad 1$ * size of this structure *

printf("size of InfoHeader Record: sd\n", dummy);

istatus $=$ fread $(\& w i d t h, 4,1, \mathrm{fp}) ; \quad /$ width in pixels * $/$

istatus $=$ fread $(\& h e i g h t, 4,1, f p) ; \quad$ * height in pixels * $/$

istatus $=$ fread $(\& p l a n e s, 2,1, f p) ; \quad / *$ planes (should be 1 ); note 2 -byte */

istatus $=$ fread $(\& b i t C o u n t, 2,1, f p) ; 1 *$ bits per pixel; note 2 -byte */ 


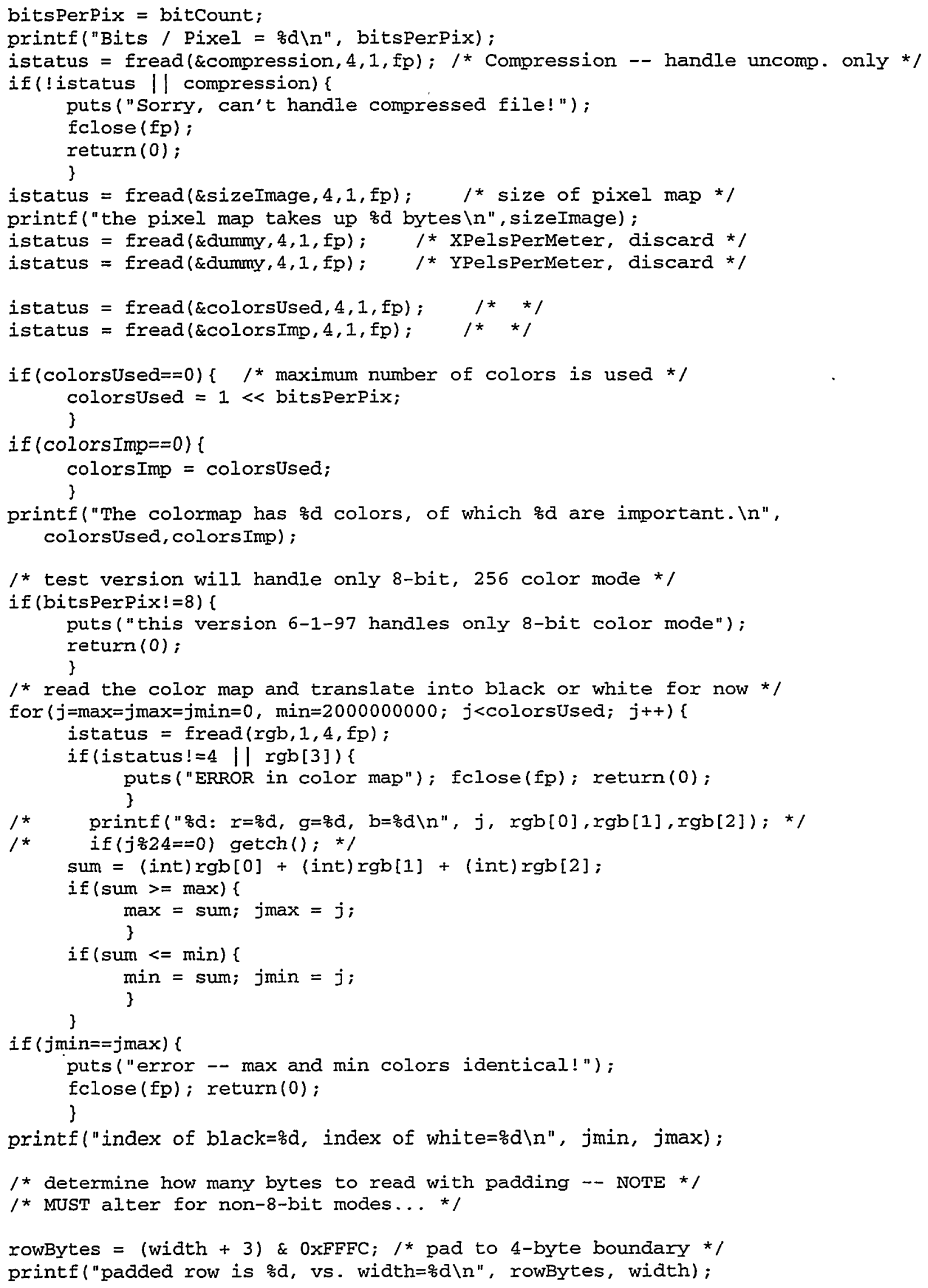




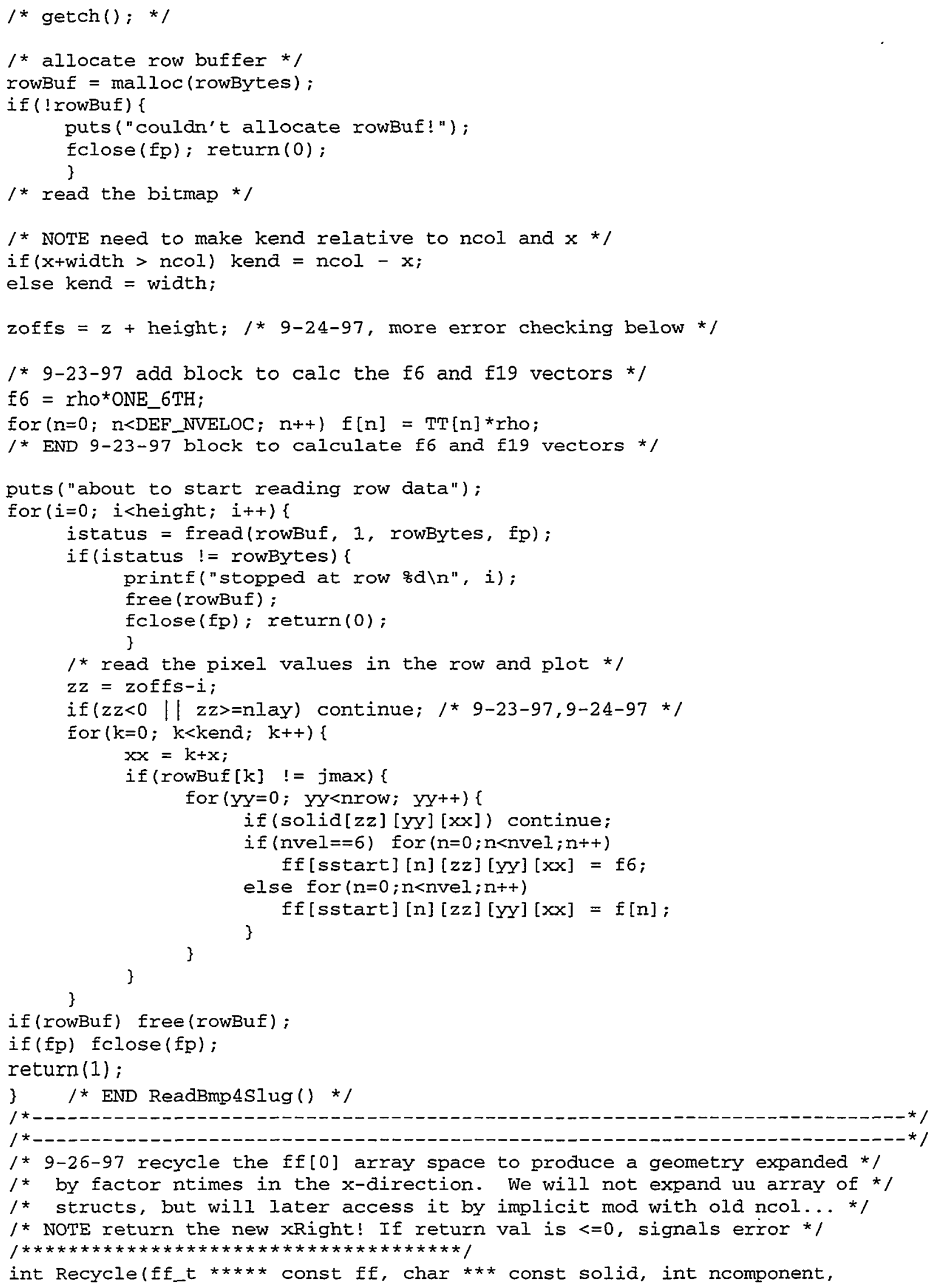


int nveloc[], int nlay, int nrow, int ncol, int ntimes, int xRight)

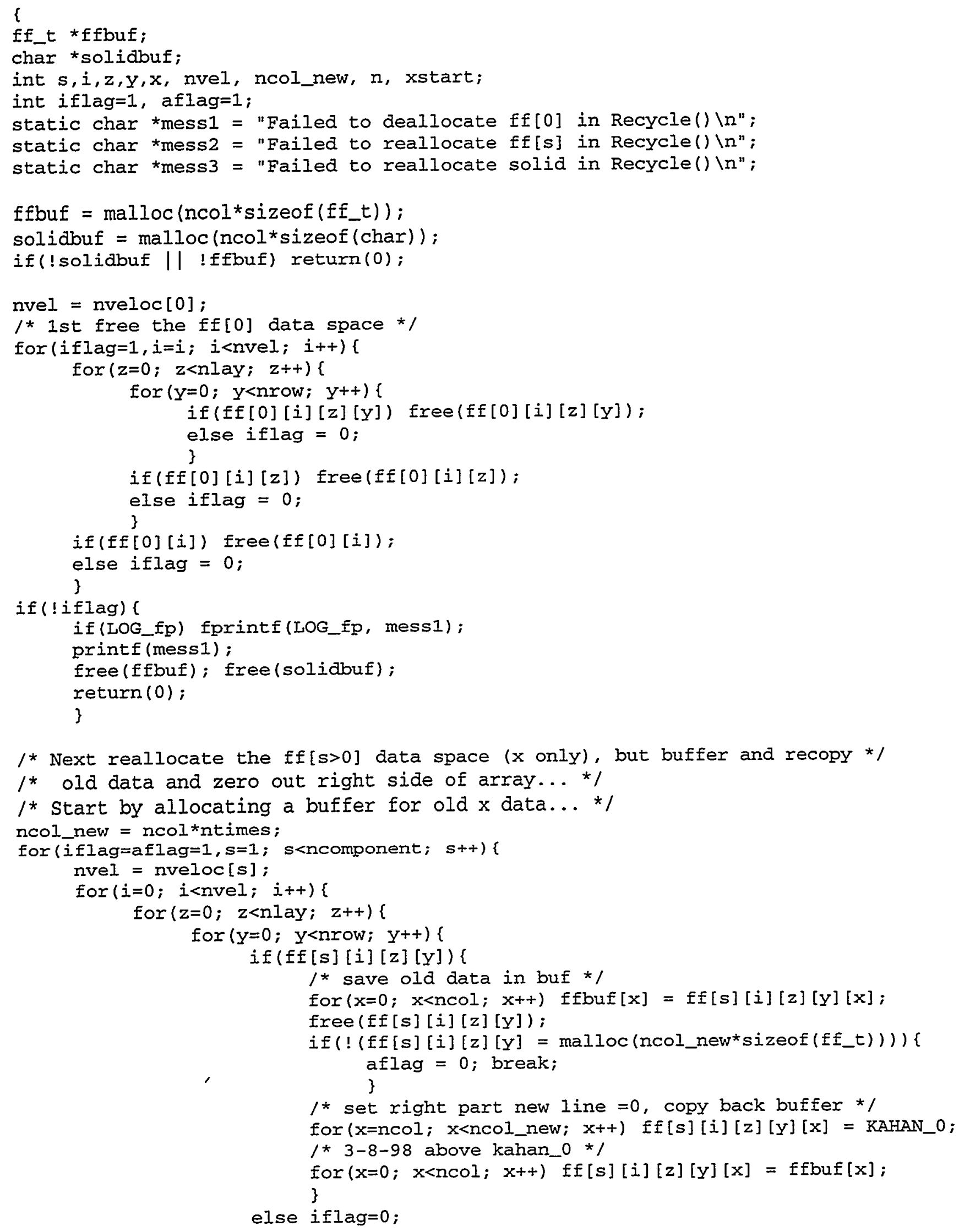




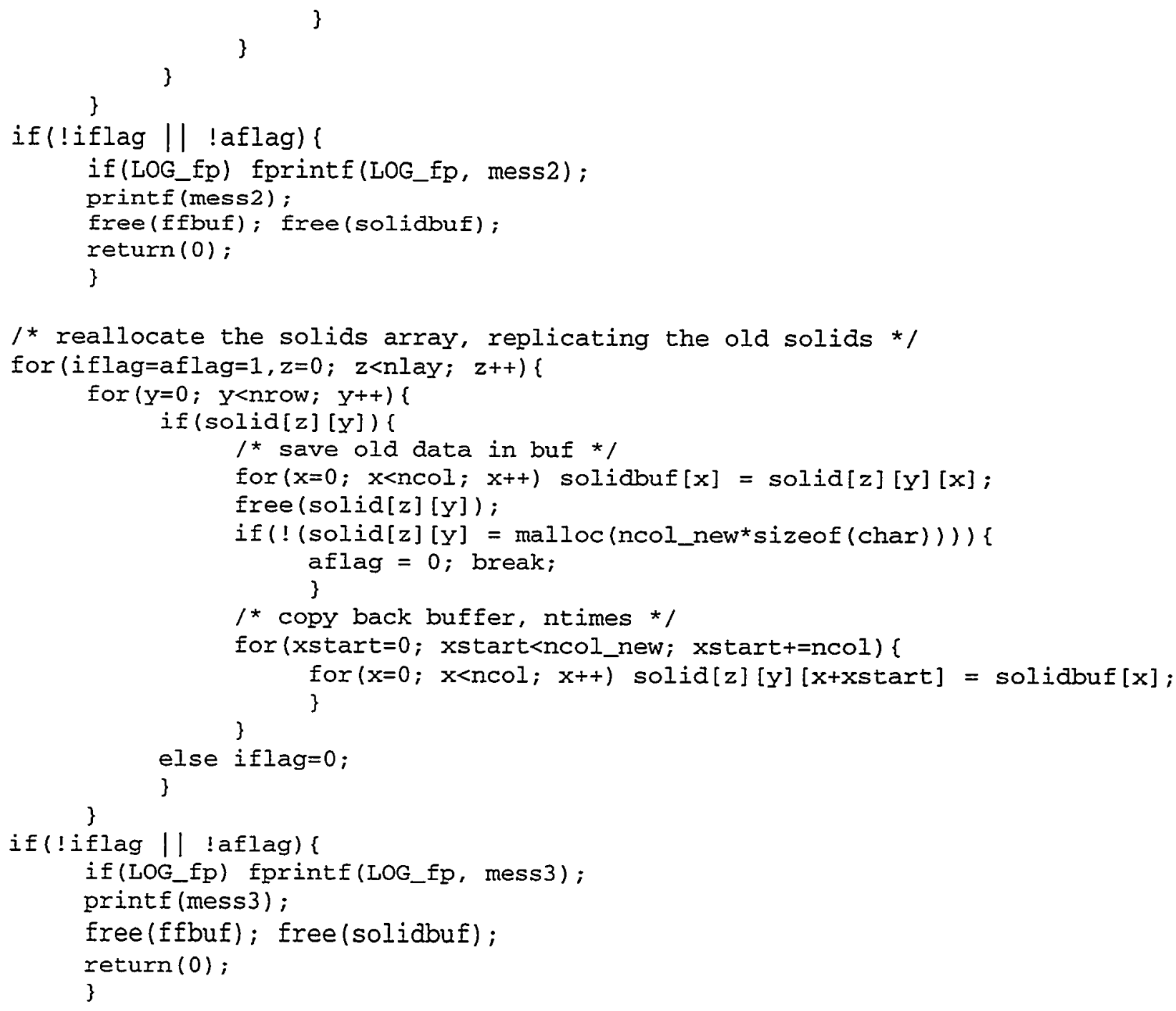


int YsetConcJB(ff_t $* * * * *$ const ff, char $* * *$ const solid,

int ncomponent, int nveloc[], int nlay, int nrow, int ncol,

\{

int $y$, int $x 1$, int $x 2$, int $z 1$, int $z 2$, double * const conclist)

int $x, z, i, s$, temp, nvel, solidcode;

1* 7-11-97 the "is_*" above test is any of the solutes are 19 or 6 vector */

/* types; would be more efficient done just once outside, but seems safer */

/* to do this simple test internally, since it will be dwarfed by fetching */

$1 *$ and computation time in rest of function, and we may some day reallocate */

1 * a 19-vector solute among 6 vectors after equilibration.... */

ff_t $f\left[D E F \_N V E L O C\right]$;

if $(y<0|| x 1<0|| x 2<0|| y>=$ nrow $\| x 1>=n c o l|| x 2>=n c o l$

||$z 1<0|| z 2<0|| z 1>$ nlay ||$z 2>$ nlay) return(0);

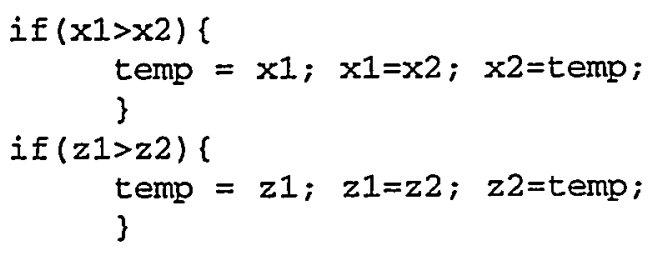




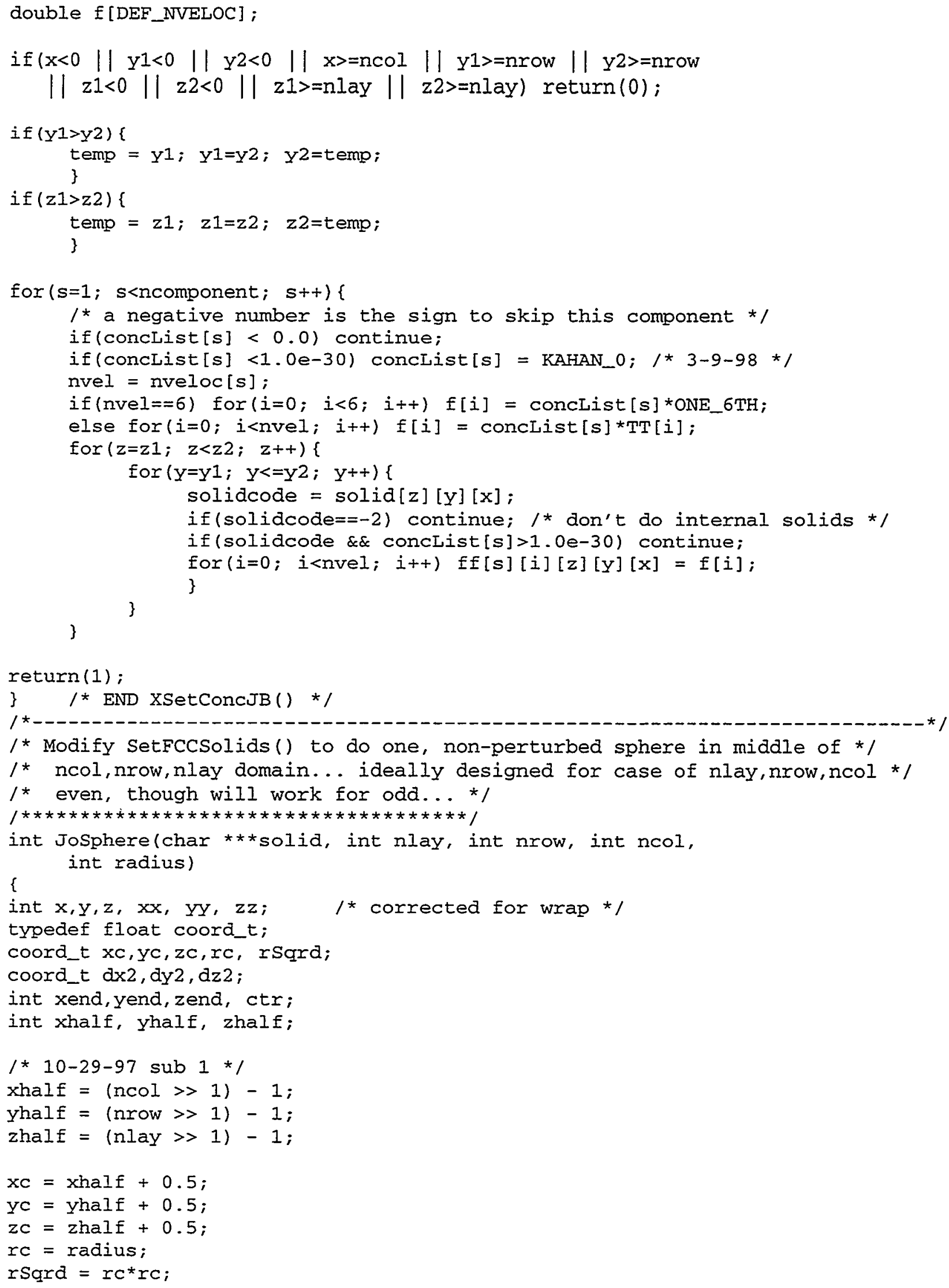




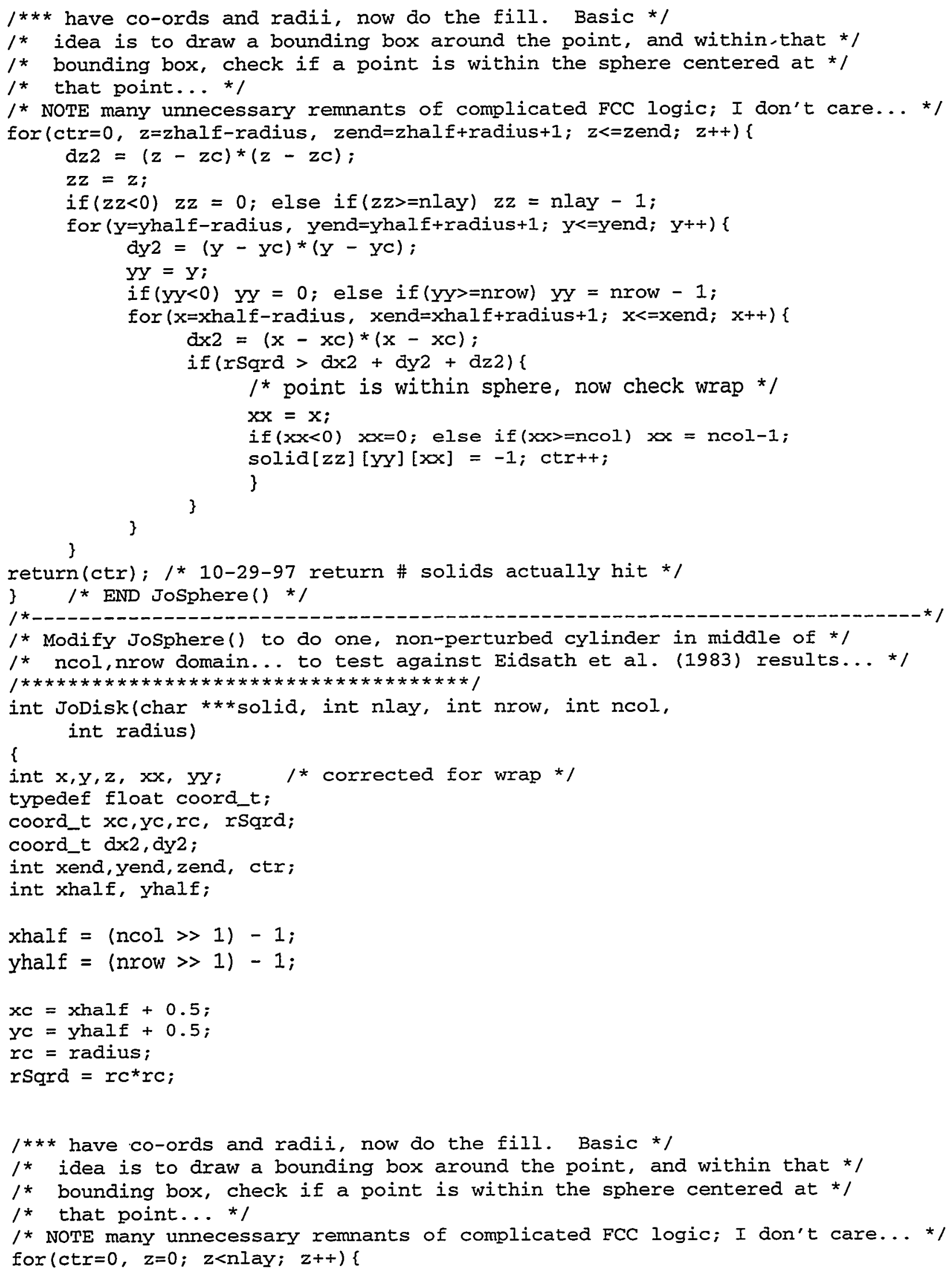


for $(y=y$ half-radius, yend=yhal + radius $+1 ; y<=y e n d ; y++)\{$ $d y^{2}=(y-y c) *(y-y c) ;$

$\mathrm{YY}=\mathrm{Y}$;

if $(y y<0)$ yy $=0$; else if (yy>=nrow) $y y=$ nrow -1 ;

for $(x=x h a l f-r a d i u s, x e n d=x h a l f+x a d i u s+1 ; x<=x e n d ; x++)\{$

$\mathrm{dx} 2=(\mathrm{x}-\mathrm{xc}) *(\mathrm{x}-\mathrm{xc}) ;$

if ( $x$ Sqrd $>d x 2+d y 2)\{$

1 * point is within sphere, now check wrap */ $\mathrm{xx}=\mathrm{x}$;

if $(\mathrm{xx}<0) \mathrm{xx}=0$; else if $(\mathrm{xx}>=\mathrm{ncol}) \mathrm{xx}=\mathrm{ncol}-1$;

solid $[z][y y][x x]=-1$; ctrt+;

\}

\}

return(ctr); / * 10-29-97 return \# solids actually hit * /

\} $\quad /$ END JoDisk() */

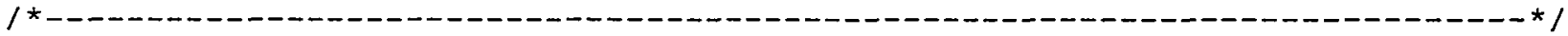

$1 * 6-13-98$ create a version for just one tracer */

$1 * 2-27-98$ pointerize to speed up? */

$l *$ 9-27-97 modify to pass ncol_old so we can find uu[z][y][xx] position.. */

$1 *$ 9-22-97 create 3D version of JustDisperse() (2D versions in Ib01.C), *

$I^{*}$ a collide function that assumes velocity field is static, therefore */

$1 *$ does not do buoyancy or carrier fluid... * $/$

1 * NOTE: for now, simplify by not using lots of pointers and by keeping */

$1 *$ ONLY bounceback mode... * /

/************************************/

int JustDisperse3D_A(ff_t $* * * * * f f$,

double * const tau, double * const beta, double *const omega,

struct veloc_t $* * *$ uu, char $* * *$ solid,

\{

int ncomponent, int nveloc[], int nlay, int nrow, int ncol, int ncol_old)

int $z, y, x, x x ; \quad / * 9-27-97 x x$ is index into uu[z][y][xx]..*/

double ux, uy, uz;

double f0, $f 1, f 2, \mp 3, f 4, \mp 5$;

ff_t *pf0,*pf1,*pf2,*pf3,*pf4,*pf5;

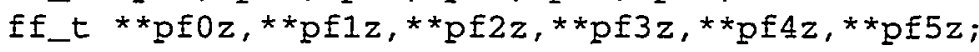

struct veloc_t **uuzp, *uup;

int solidcode;

unsigned char **solidzp, *solidp;

double rho, beta_s;

double mux, muy, muz, mul;

1 * const static double half $=$ ONE_HALF; * $/$

double halfomega, sixthomega; / * 6-13-98*/

if (ncomponent!=2 || nveloc[1] !=6) \{

puts ("incorrect JustDisperse3D_A() function");

exit (-11);

\}

halfomega $=$ ONE_HALF ${ }^{*}$ omega [1];

sixthomega $=$ ONE_6TH*omega [1];

beta_s = beta [1];

for $(z=0 ; \quad z<$ nlay; $z++)($ 


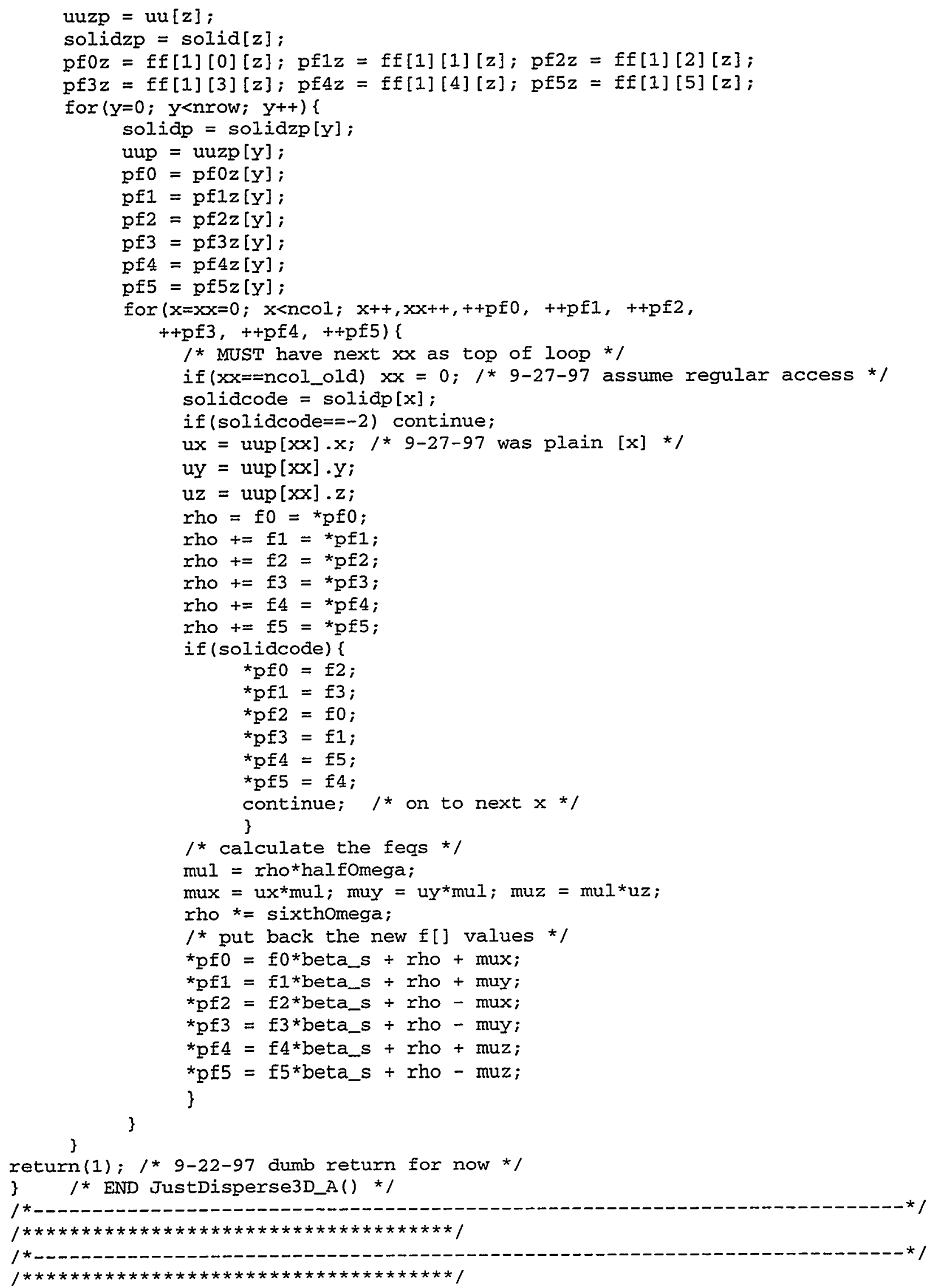




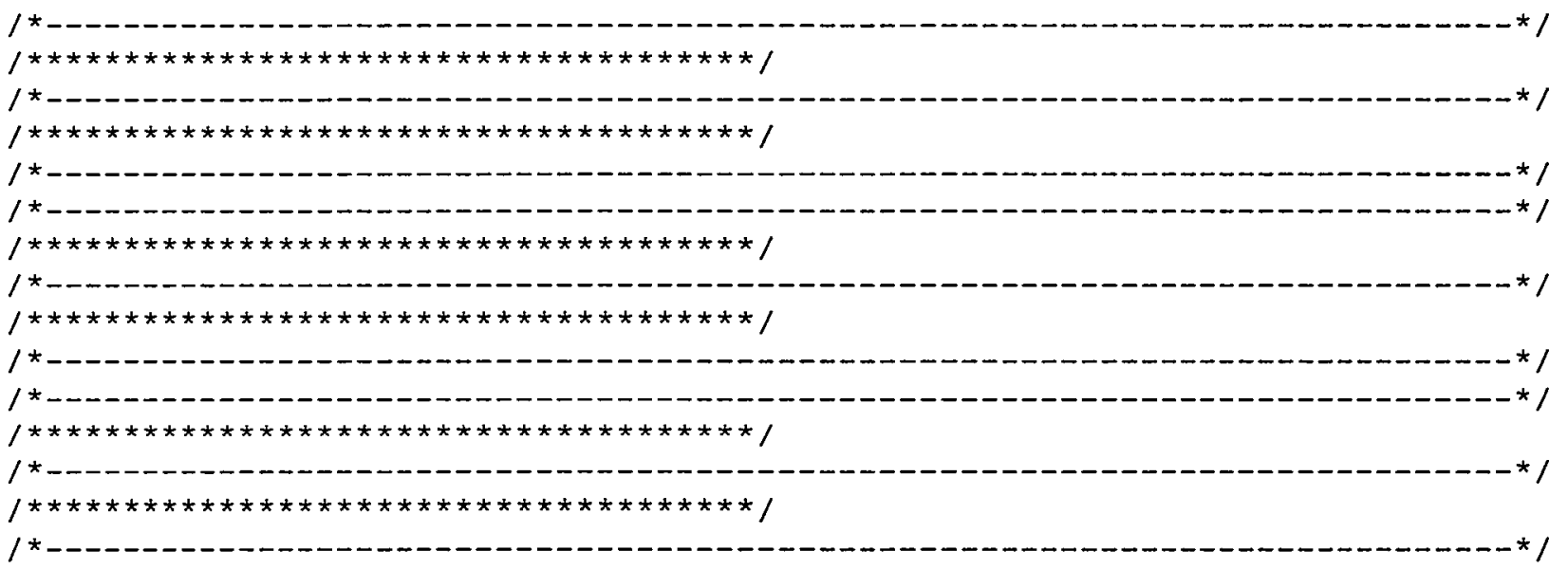




\section{Distribution List:}

Iran Thomas

Deputy Associate Director

U.S. Department of Energy

19901 Germantown Rd.

Germantown, MD 20874-1290

Dr. Nicholas Woodward

$\mathrm{BES} /$ Division of Engineering and

Geoscience

ER-15

U.S. Department of Energy

19901 Germantown Road

Germantown, MD 20874-1290

Paula M. Davidson

Geoscience Research Program, ER-15

Office of Basic Energy Sciences

U.S. Department of Energy

19901 Germantown Rd.

Germantown, MD 20874-1290

Harlan Stockman

1261 Town Center Drive

Mailstop 423

Las Vegas, NV 89134
MS 0419 C. E. Meyers, 4000

MS 0701 L. E. Shephard, 6100

MS 0735 P. B. Davies, 6115

MS 0735 R. J. Glass, 6115

MS 0750 H. R. Westrich, 6118

MS 0751 J. T. Fredrich, 6117

MS 0751 L. S. Costin, 6117

MS 9402 C. M. Hartwig, 8701

MS 0841 P. J. Hommert, 9100

MS 0826 D. R. Noble, 9111

MS 0826 W. L. Hermina, 9111

MS 0835 S. N. Kempka, 9113

MS 1111 J. N. Shadid, 9221

MS 1111 G. S. Heffelfinger, 9225

MS 9019 Central Technical Files, (1) 8940-2

MS 0899 Technical Library, (2) 4916

MS 0619 Review and Approval (1)

Desk, 15102

For DOE/OSTI 